\title{
Investigations in Molecular Recognition Element Selection and Single-Wall Carbon Nanotube Properties
}

\author{
Ryan Martin Williams \\ West Virginia University
}

Follow this and additional works at: https://researchrepository.wvu.edu/etd

\section{Recommended Citation}

Williams, Ryan Martin, "Investigations in Molecular Recognition Element Selection and Single-Wall Carbon Nanotube Properties" (2013). Graduate Theses, Dissertations, and Problem Reports. 5011.

https://researchrepository.wvu.edu/etd/5011

This Dissertation is protected by copyright and/or related rights. It has been brought to you by the The Research Repository @ WVU with permission from the rights-holder(s). You are free to use this Dissertation in any way that is permitted by the copyright and related rights legislation that applies to your use. For other uses you must obtain permission from the rights-holder(s) directly, unless additional rights are indicated by a Creative Commons license in the record and/ or on the work itself. This Dissertation has been accepted for inclusion in WVU Graduate Theses, Dissertations, and Problem Reports collection by an authorized administrator of The Research Repository @ WVU.

For more information, please contact researchrepository@mail.wvu.edu. 


\section{Investigations in Molecular Recognition Element Selection and Single-Wall Carbon Nanotube Properties}

\section{Ryan Martin Williams}

A dissertation submitted to the School of Pharmacy at West Virginia University in partial fulfillment of the requirements for the degree of

Doctor of Philosophy in Pharmaceutical and Pharmacological Sciences

Letha J. Sooter, Ph.D., Chair Peter Gannett, Ph.D.

Yon Rojanasakul, Ph.D.

Linda Vona-Davis, Ph.D. Clifton Bishop, Ph.D.

Department of Basic Pharmaceutical Sciences

Morgantown, West Virginia

2013

Keywords: Molecular Recognition Element, In vitro selection, SELEX, Aptamer, Prostate Cancer, Pesticides, Carbon Nanotubes, Single-Wall Carbon Nanotubes, SWCNT, Polymerase Chain Reaction, PCR, Toxicology 


\title{
$\underline{\text { Abstract }}$ \\ Investigations in Molecular Recognition Element Selection and Single-Wall Carbon Nanotube Properties
}

\author{
Ryan Martin Williams
}

The in vitro selection of Molecular Recognition Elements (MREs) is a powerful tool for identifying molecules useful in numerous applications. This is achieved using the iterative Systematic Evolution of Ligands by EXponential Enrichment (SELEX) process. This technique utilizes a large library of $10^{9}-10^{15}$ different molecules which is enriched for those that bind to a target of interest. It also can be designed to enrich for molecules which do not bind to other, closely-related negative targets. We have developed a novel variation of this process called Decoy-SELEX. This enriches molecules that bind to the target of interest, but focuses on what the MRE should not bind to by using highly stringent negative selections with multiple targets. We have used this novel technique to identify separate MREs for three targets. First, a singlechain Fragment variable (scFv) antibody fragment MRE was identified that binds to prostate cancer cells but not benign prostatic cells. This molecule will be useful in the specific detection and targeted therapy of prostate cancer. Then, a single-stranded DNA (ssDNA) MRE was identified that binds to the herbicide atrazine but not other closely-related molecules. This molecule will be useful in the rapid, field-use detection of environmental contamination by atrazine. Finally, a ssDNA MRE binding to the pesticide malathion but not its metabolites was obtained. This molecule will also be useful as an environmental sensor for malathion contamination.

Single-wall carbon nanotubes (SWCNT) are a relatively new class of materials with novel mechanical, electrical, and optical properties. These properties are dependent upon the chirality and length of individual tubes, and can be altered with modifications, including chemical conjugates, DNA-wrapping, or protein adsorption. Therefore, SWCNT have many potential uses, including incorporation into electrical or optical molecular sensors. In order to determine the future potential of DNA-wrapped SWCNT in sensing applications, chirality identification, and purification, we tested their effect on the polymerase chain reaction (PCR). We determined the reaction-limiting concentration for different DNA sequences and SWCNT chiralities in addition to determining that DNA can be PCR-amplified directly from a SWCNT scaffold. This study identified parameters important in future studies for SWCNT application. Additionally, we determined the effect of DNA-wrapped SWCNT on a green algae model organism compared to sodium cholate-wrapped SWCNT. We determined that there was no effect of either type of SWCNT on algal growth at the concentrations assayed. This is important as the manufacture and application of SWCNT increases, making the likelihood of environmental exposure more prominent. 


\section{Dedicated to}

My parents, David and Jackie Williams, for shaping everything about what I have and will become

My sister, Mary Grace Williams, for friendship and happiness

My grandparents and close family members for having more influence than they know

My close friends for positivity and support 


\section{Acknowledgements}

I want to thank my advisor, Dr. Letha Sooter, for her strong support and the opportunity to succeed.

My committee members also deserve my gratitude for their guidance and helpfulness throughout my work. Dr. Peter Gannet, Dr. Yon Rojanasakul, Dr. Linda Vona-Davis, and Dr. Clif Bishop have been tremendously helpful.

All current and former members of the Sooter Lab should be thanked for their assistance, thoughts, and friendship. Special thanks are extended to those who helped with the work described here: Dr. Bridget Dolash, Sara Nayeem, Cyrus Hajiran, Jackson Thomas, Hannah Taylor, Ginger He, Luisa Battistella, and Eamonn Maher.

All other faculty, staff, and students of the Departments of Basic Pharmaceutical Sciences and Biology, as well as other programs I have been involved with, have been a positive constant throughout my graduate work.

I also want to thank Amanda Kulick for her help, for dealing with a Ph.D. student, and for keeping me sane.

I am extremely grateful to the American Foundation for Pharmaceutical Education and NanoSAFE/WVNano for the last two years of fellowship support. Each of these has been instrumental in allowing me to expand my education. 


\section{TABLE OF CONTENTS}

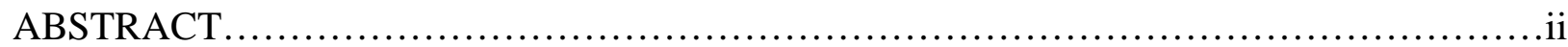

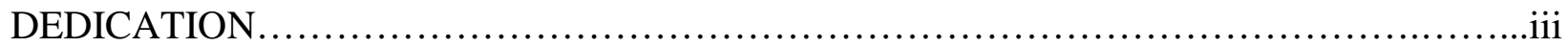

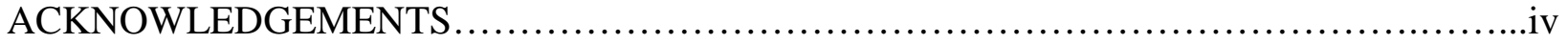

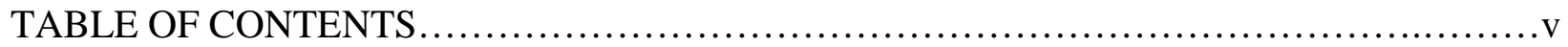

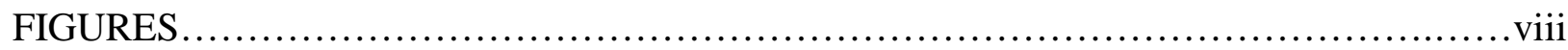

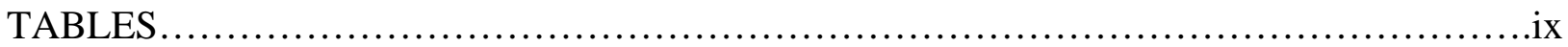

CHAPTER 1. Introduction to In Vitro Selection of Molecular Recognition Elements.........1

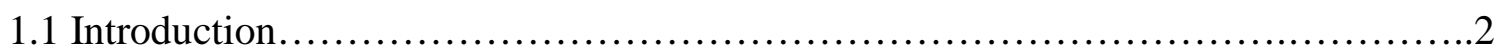

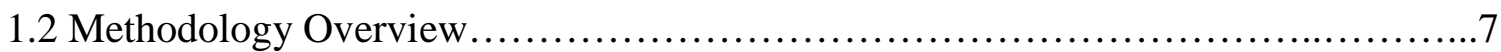

1.2.1 Amino Acid Library Creation.............................................

1.2.1.1 Phage Display...............................................

1.2.1.2 Bacterial Display.............................................. 8

1.2.1.3 Yeast Display..................................................

1.2.2 Nucleic Acid Library Creation.........................................10

1.2.3 Amino Acid Separation Methods...........................................11

1.2.4 Nucleic Acid Separation Methods.........................................14

1.3 Amino Acid Molecular Recognition Elements for Cell Surface Targets...............15

1.3.1 Peptide MREs............................................................

1.3.2 Antibody Fragment MREs.............................................17

1.4 Small Molecule Nucleic Acid Molecular Recognition Elements.......................18

1.4.1 DNA MREs........................................................ 18

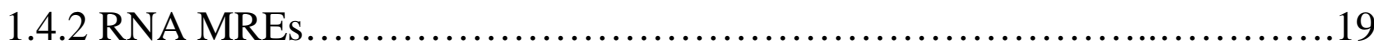

1.5 Molecular Recognition Element Applications....................................20

1.5.1 Amino Acid Molecular Recognition Element Applications..................20

1.5.1.1 Therapeutics...................................................20

1.5.1.2 Disease Detection..........................................22

1.5.1.3 Surface Molecule Elucidation and Discovery.....................23

1.5.2 Nucleic Acid Molecular Recognition Element Applications.................23

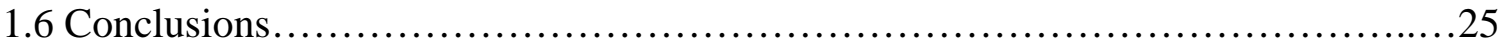

\section{CHAPTER 2. In Vitro Selection of Single-Stranded DNA Molecular Recognition Elements \\ for the Herbicide Atrazine................................................................27}

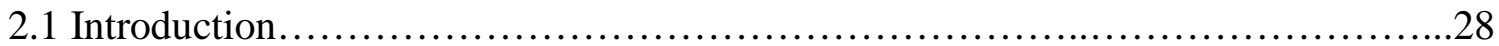

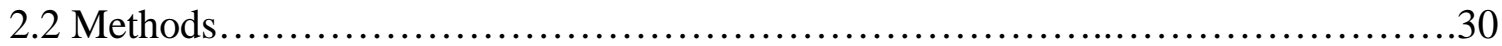

2.2.1 Selection of Atrazine-Specific MREs..................................30

2.2.2 Sequencing of Atrazine-Specific MREs..................................35

2.2.3 Atrazine MRE Binding Assays...........................................35

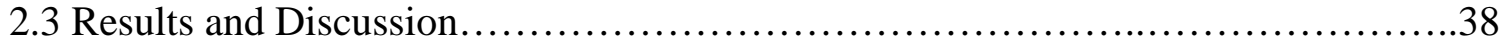

2.3.1 Selection of Atrazine-Specific MREs..................................38

2.3.2 Affinity and Specificity of Atrazine-Specific MRE.......................40

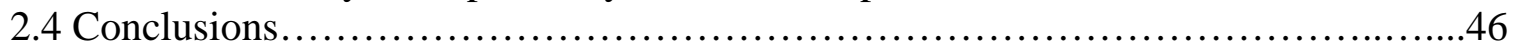


CHAPTER 3. Introduction to the Properties and Applications of Single-Wall Carbon Nanotubes.....................................................................................47

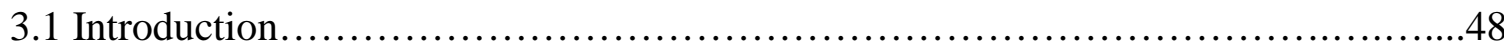

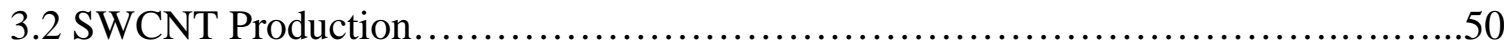

3.2.1 Arc-Discharge Method.................................................50

3.2.2 Laser-Ablation Methods.............................................50

3.2.3 Chemical Vapor Deposition Method......................................51

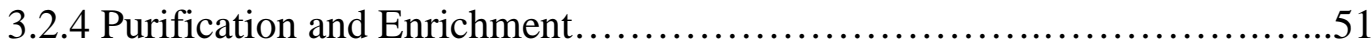

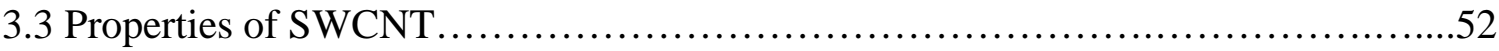

3.3.1 Mechanical Properties...................................................53

3.3.2 Electrical Properties................................................54

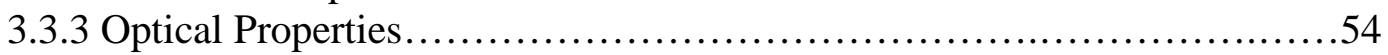

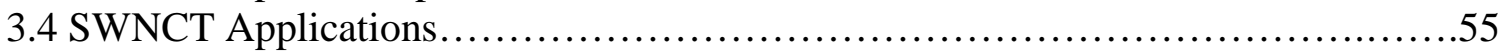

3.4.1 Industrial Applications................................................55

3.4.2 Electronic Applications..........................................56

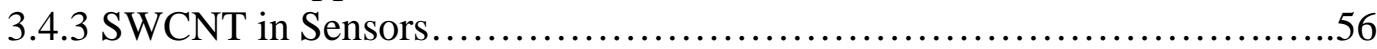

3.4.4 Biomedical Applications.............................................57

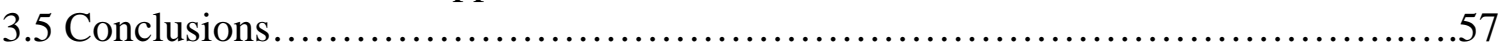

CHAPTER 4. Investigation of the Effects of DNA-dispersed Single-Wall Carbon Nanotubes on the Polymerase Chain Reaction...............................................59

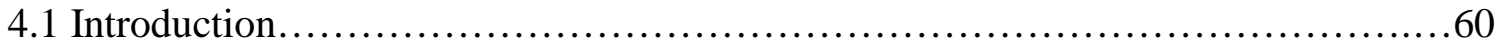

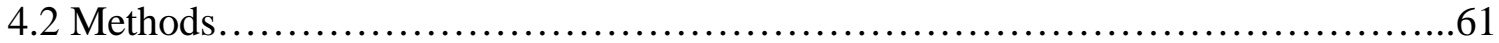

4.2.1 DNA-Dispersed SWCNT Preparation....................................61

4.2.2 SWCNT Characterization.............................................62

4.2.3 Polymerase Chain Reaction in the Presence of DNA-Dispersed SWCNT...62

4.2.4 PCR Analysis by Agarose Gel Electrophoresis...........................63

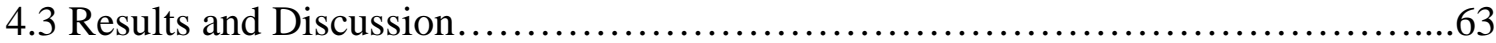

4.3.1 Analysis of DNA:SWCNT Preparations.................................63

4.3.2 Agarose Gel Analysis of Old DNA:SWCNT PCRs......................64

4.3.3 Agarose Gel Analysis of New DNA:SWCNT PCRs.....................67

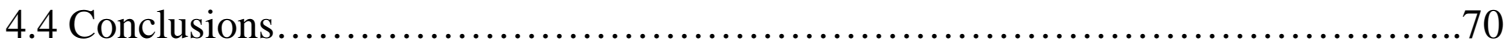

\section{CHAPTER 5. Investigation of the Effects of DNA-Dispersed Single-Wall Carbon Nanotubes and Sodium Cholate-Dispersed Single-Wall Carbon Nanotubes on the Green Algae Chlamydomonas reinhardtii............................................................72}

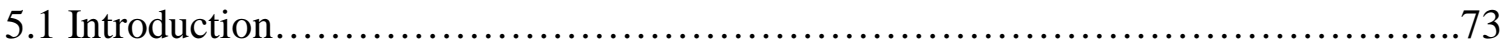

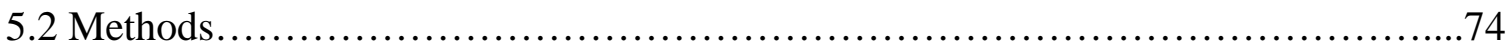

5.2.1 SWCNT Preparation................................................. 74

5.2.2 SWCNT Characterization............................................. 75

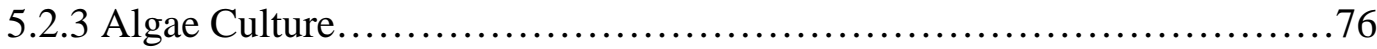

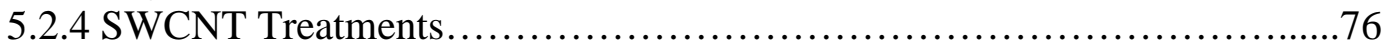


5.2 .5 Growth Measurements..............................................76

5.3 Results and Discussion.................................................. 77

5.3.1 SWCNT Preparation and Characterization..............................77

5.3.2 Effects of SWCNT and Dispersion Method on Algal Growth...............79

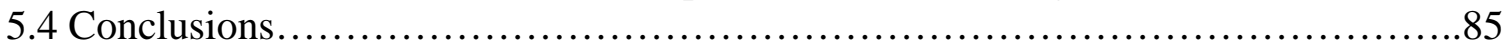

\section{Appendix A. In Vitro Selection of Antibody Fragment Molecular Recognition Elements for Prostate Cancer Cells.....................................................................86}

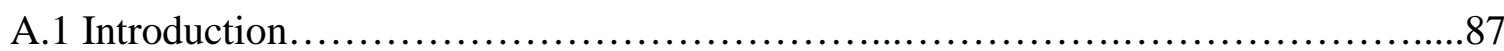

A.2 Methods.................................................................... 91

A.2.1 Cell Culture and Materials.............................................92

A.2.2 scFv Library and Growth............................................94

A.2.3 In Vitro Selection...................................................95

A.2.4 Fluorescent Imaging of scFv Library Enrichment........................98

A.2.5 Sequencing of scFv Library ....................................... 99

A.2.6 Secretion and Purification of Selected scFvs............................100

A.2.7 Affinity Binding Assays with scFvs..................................102

A.2.8 Specificity Binding Assays of scFvs................................. 103

A.2.9 Fluorescent Imaging of $\mathrm{scFv}$ Binding.............................. 105

A.3 Results and Discussion....................................................

A.3.1 Selection of MREs...............................................105

A.3.2 Affinity Binding Assays of scFvs..................................... 109

A.3.3 Specificity Binding Assays of scFvs.................................111

A.4 Conclusions ........................................................... 116

\section{Appendix B. In Vitro Selection of Single-Stranded DNA Molecular Recognition Elements}

for the Pesticide Malathion.............................................................117

B.1 Introduction.............................................................118

B.2 Methods............................................................... 121

B.2.1 Decoy-SELEX for Malathion-Specific MREs.........................121

B.2.2 Sequencing of the Enriched ssDNA Library..........................126

B.2.3 Determination of MRE Binding Affinity..............................128

B.3 Results and Discussion................................................

B.3.1 Identification of Malathion-Specific MRE............................129

B.3.2 Affinity and Specificity of Malathion-Specific MRE.............................131

B.4 Conclusions.......................................................134

References.........................................................................................136

Curriculum Vitae........................................................................175 


\section{LIST OF FIGURES}

Figure 1.1. Visual depiction of Molecular Recognition Element (MRE) binding..................2

Figure 1.2. Scheme depicting the iterative SELEX process................................

Figure 2.1. Scheme depicting the Decoy-SELEX process for atrazine MRE selection...........31

Figure 2.2. Chemical structures of molecules used in the Decoy-SELEX scheme and cross-

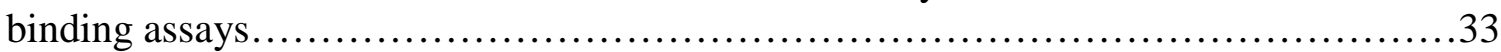

Figure 2.3. Sequences and structures of atrazine MREs....................................39

Figure 2.4. Fluorescent equilibrium binding assay of R12.23............................41

Figure 2.5. Sequence and structure of R12.23.TRNC1 .................................42

Figure 2.6. Cross-binding studies of R12.23 atrazine MRE to other molecules.................43

Figure 3.1. Derivation of SWCNT $(n, m)$ chiral species...................................49

Figure 4.1. Agarose gel electrophoresis images and analysis of PCRs with old (4-10 months .

DNA:SWCNT samples...................................................65

Figure 4.2. Agarose gel electrophoresis images and analysis of PCRs with new ( $<1$ month)

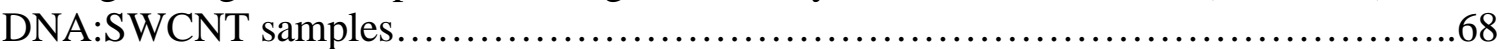

Figure 5.1. Characterization of DNA:SWCNT and SC:SWCNT .........................78

Figure 5.2. Ten day growth curve of Chlamydomonas reinhardtii with DNA:SWCNT treatments

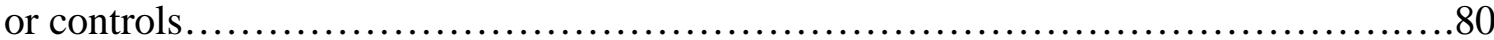

Figure 5.3. Ten day growth curve of Chlamydomonas reinhardtii with SC:SWCNT treatments or

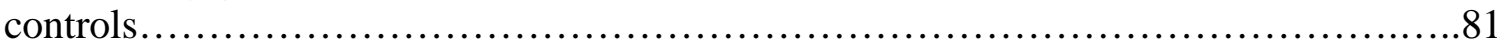

Figure 5.4. Extracted Chlorophyll $\beta$ content at OD 663 for DNA:SWCNT and SC:SWCNT

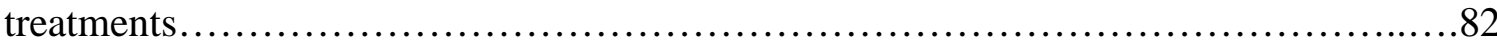

Figure 5.5. Extracted Chlorophyll $\alpha$ content at OD 645 for DNA:SWCNT and SC:SWCNT

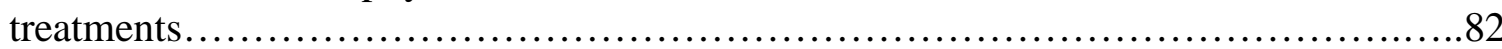

Figure 5.6. Micrograph of algae grown with SWCNT ...................................84

Figure A.1. Scheme depicting the Decoy-SELEX process for LNCaP MRE selection..........91

Figure A.2. Enrichment of the Lo and Hi yeast-displayed scFv populations after FACS-based

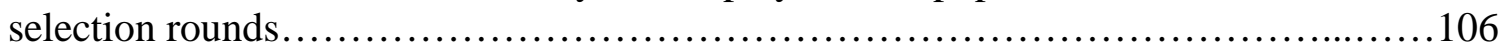

Figure A.3. Fluorescent micrographs depicting yeast binding enrichment...................107

Figure A.4. Plot of fluorescent equilibrium binding assay of HiR6.8 and LNCaP cells.........110

Figure A.5. Micrographs of scFv binding to LNCaP cells...............................112

Figure A.6. Cross-binding of HiR6.8 to negative targets and other cell lines..................114

Figure A.7. Cross-Binding of HiR7.8 to negative targets and other cell lines..................114

Figure B.1. Decoy-SELEX scheme for selection of a malathion MRE.....................122

Figure B.2. Chemical structures of molecules used in selection of a malathion-specific MRE.123

Figure B.3. Sequences of malathion MREs chosen from Round 12 library...................130

Figure B.4. Fluorescence equilibrium binding assays of R12.20 for malathion........................132

Figure B.5. Cross-binding studies of malathion MRE to other molecules used in the selection.133 


\section{LIST OF TABLES}

Table 1.1. Summary of the advantages of the four most typical types of MRE libraries.........6

Table 1.2. Typical methods of separation in SELEX ..................................... 12

Table 2.1. Decoy-SELEX scheme for atrazine MRE selection.............................37

Table 2.2. Equilibrium dissociation constant $\left(\mathrm{K}_{\mathrm{d}}\right)$ of atrazine MREs.......................41

Table 3.1. Special properties of single-wall carbon nanotubes (SWCNT) and their correlation

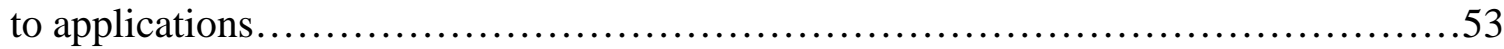

Table 4.1. Synthesized DNA sequences used in DNA:SWCNT PCRs........................62

Table A.1. Decoy-SELEX scheme for identification of prostate cancer cell-specific MREs......93

Table A.2. Amino acid sequence of selected prostate cancer cell MREs.....................109

Table A.3. Equilibrium dissociation constant $\left(\mathrm{K}_{\mathrm{d}}\right)$ of prostate cancer cell MREs...............110

Table B.1. Decoy-SELEX outline for identification of a malathion MRE....................127 


\section{CHAPTER 1}

Introduction to In Vitro Selection of Molecular Recognition Elements 


\subsection{Introduction}

The Selective Evolution of Ligands by EXponential Enrichment (SELEX) is a method of obtaining molecular recognition elements (MREs) that bind to a target of interest (Figure 1.1). This in vitro process is a powerful tool for selecting molecules useful in the specific detection or treatment of targets of interest. Selectivity for a certain molecule or disease state can be ensured in designing the experiment with expected use of the final product being considered.

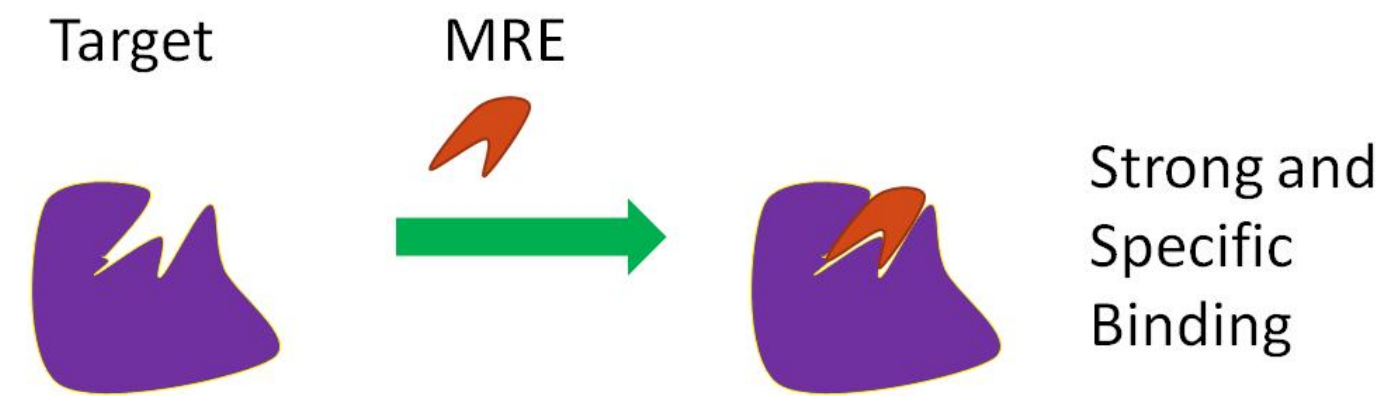

Figure 1.1. Visual depiction of Molecular Recognition Element (MRE) binding. A MRE is any molecule with strong and specific binding to a target of interest.

The SELEX, or in vitro selection, method obtains one or a few molecules that bind to the target with high affinity and specificity. These MREs are selected from a large library of $10^{7}$ $10^{15}$ random molecules. SELEX was originally described independently by the laboratories of Gold and Szostak in 1990 (Ellington and Szostak 1990; Tuerk and Gold 1990). These experiments selected RNA MREs that bind to proteins. Both ribonucleic acid (RNA) and deoxyribonucleic acid (DNA) MREs have been selected that recognize a range of targets, from single ions and small molecules to nanoparticles and proteins (Paborsky et al. 1993; Breaker and Joyce 1994; Huizenga and Szostak 1995; Gugliotti et al. 2004). These molecules are typically referred to in the literature as aptamers. The same SELEX method has been used to develop antibody fragment and peptide MREs that bind to small molecules, proteins, nucleic acids, and nanoparticles (Boder et al. 2000; Schaffitzel et al. 2001; El-Mousawi et al. 2003; Kase et al. 
2004). The wide variety of MRE libraries and potential targets speaks to the numerous applications of SELEX.

The SELEX process uses a large library of random molecules that are incubated with the target of interest (Figure 1.2). Those molecules that bind to this target are kept and amplified, while those that don't are discarded. The amplified binding molecules are then incubated with negative targets, something the final MRE is designed to not bind to. Those molecules that do bind these targets are discarded and those that don't are amplified and re-subjected to the target of interest. This process of targeting and negative targeting is performed (rounds of selection) until one or a few high-affinity molecules remain in the library. The selected MREs are then

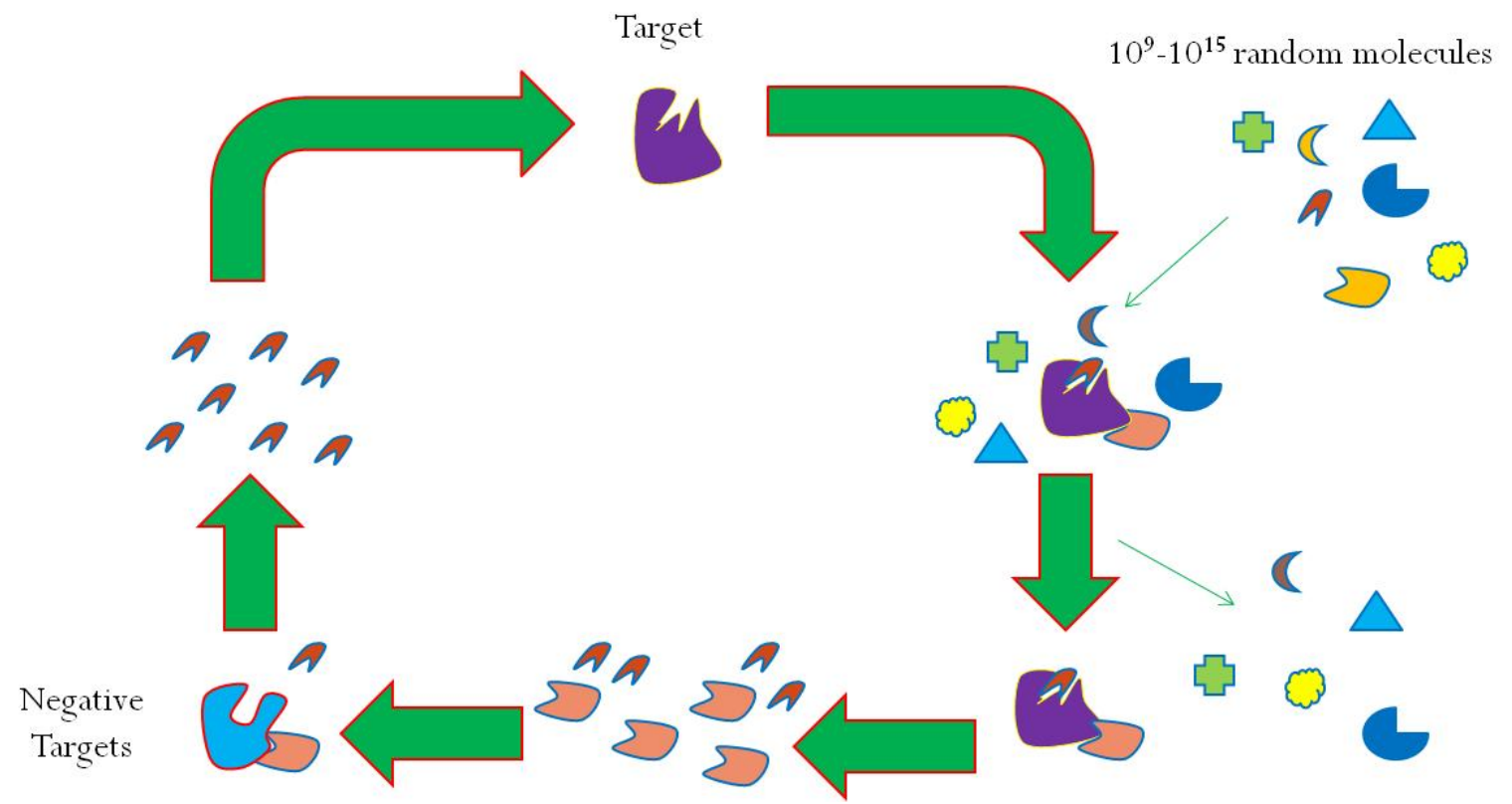

Figure 1.2. Scheme depicting the iterative SELEX process. A large pool of random molecules is incubated with the target of interest. Non-bound molecules are washed away while bound are amplified and subjected to negative targets. Non-bound molecules are amplified and re-subjected to the target under more stringent conditions.

assayed for their binding affinity and selectivity before they are used for various applications. Regarding amino acid libraries, amplification typically occurs by use of a cellular host. For DNA and RNA libraries, amplification occurs via the polymerase chain reaction (PCR). 
Molecular recognition elements have been selected that bind to molecules displayed on the surface of cells. Originally performed by the Gold laboratory, DNA MREs that bind to the surface of red blood cells were selected (Morris et al. 1998). This work showed the ability to select MREs that bind to molecules displayed on cell surface membranes in their natural state. Since that time, SELEX has been used to select MREs that bind to cell surface molecules that are differentially expressed on the surface of cells. DNA ligands have been selected that bind to the vasculature of rat brain glioblastoma and not normal rat brain vasculature (Blank et al. 2001). Other nucleic acid MREs have been selected that bind to molecules on the surface of cancer cells but not other types of cells (Shangguan et al. 2008; Sefah et al. 2009; Zhao et al. 2009). This type of MRE selection provides the dual benefits of binding to anchored molecules and taking advantage of differences in surface expression of cells for the therapy and diagnosis of disease. Compared to DNA and RNA MREs, amino acid-based MREs potentially provide the stability and specificity of antibodies in vivo (Table 1.1) (Nelson 2010; Li et al. 2011). Additionally, the non-reversible nature of their target interaction and high binding affinity is an advantage in tumor cell targeting. While having the positive attributes of antibodies in vivo, these MREs are selected in vitro. Therefore, more control over their binding target is possible for the experimenter. This includes the ability to preferentially select molecules which bind to a target but not a closely-related target. In the case of differential cell SELEX, differential surface molecule expression is taken advantage of for MRE binding (Cerchia et al. 2009). The result of amino acid MRE selection using differential cell SELEX is a molecule that has the advantages of antibodies with tunability in the experimental design.

Nucleic acid MREs have been investigated for their potential as in vivo diagnostic and therapeutic tools (Cerchia et al. 2002; Ireson and Kelland 2006; Lee et al. 2006; Guo et al. 2008; 
Kim et al. 2009). It is possible that they will be degraded in the body by nucleases, however there are modification strategies to overcome this (Kanwar et al. 2011). In fact, Macugen (pegaptanib) is an anti-vascular endothelial growth factor (VEGF) nucleic acid MRE currently used in the clinic for macular degeneration (Moshfeghi and Puliafito 2005). This drug, however, is only used in the relatively closed environment of the eye, while Avastin (bevacizumab) is an anti-VEGF antibody used in anti-cancer therapy (Jain et al. 2006). Thus, there are modifications for using nucleic acid MREs in vivo, but amino acid MREs may be more useful.

Despite limited use in vivo, nucleic acid MREs have great potential for molecular detection in a variety of other settings. This is due to their typically strong affinity and selectivity, as well as their ability to be re-used. They can be denatured to release the target of interest after a binding event, and regenerated for further detection of the analyte (Ocana et al. 2012). Additionally, it is possible that nucleic acid MREs change their conformation upon binding their target, which can be capitalized on for detection transduction (Liu et al. 2010). Therefore, DNA and RNA MREs have a great deal of potential that is not found with antibodies or amino acid MREs.

There are advantages and disadvantages for DNA MREs compared to RNA MREs. Reports have shown that RNA MREs have a higher affinity for their target in general than DNA MREs (Potty et al. 2011). This is likely due to the inclusion of a 2' hydroxyl in the ribose backbone of RNA. However, nucleases that target RNA are much more abundant than those that target DNA, and thus identifying and applying RNA MREs is much more difficult. Additionally, PCR amplification of RNA is more difficult, as it must first be reverse-transcribed to DNA beforehand. Thus, DNA MREs typically are more stable and suitable for most applications, though RNA MREs do have value. 
Discussed here is an overview on the creation of both amino acid and nucleic acid libraries for SELEX experiments. Then, selections for different types of each and finally, their applications, are investigated. Selection of antibody fragments for cell surface molecules and of nucleic acids for small molecules is focused upon.

Table 1.1. Summary of the advantages of the four most typical types of MRE libraries.

\begin{tabular}{|c|c|c|}
\hline Type of Library & $\begin{array}{l}\text { Library } \\
\text { Subtype }\end{array}$ & Advantages \\
\hline \multirow[t]{7}{*}{ Amino Acid } & & -In vivo stability \\
\hline & & -Diversity of 20 amino acids, \\
\hline & Peptide & -Well-studied \\
\hline & & -Widely available libraries \\
\hline & Antibody & -Antibody structure and diversity selectable in vitro \\
\hline & & -Final product can be made into a full antibody \\
\hline & & $\begin{array}{l}\text {-Pharmacological profile similar to antibodies which are } \\
\text { clinically available }\end{array}$ \\
\hline \multirow[t]{8}{*}{ Nucleic Acid } & & $\begin{array}{l}\text {-Large and diverse libraries easily obtained through } \\
\text { combinatorial chemistry }\end{array}$ \\
\hline & & $\begin{array}{l}\text {-Easily distributable (only need to know sequence to make } \\
\text { your own) }\end{array}$ \\
\hline & & $\begin{array}{l}\text {-Re-usable (can be removed from a target and regenerated for } \\
\text { future use) }\end{array}$ \\
\hline & & -Easily modifiable \\
\hline & DNA & -Easier to work with and less degradable than RNA \\
\hline & & -Very useful in molecular detection \\
\hline & RNA & -Typically higher affinity than DNA \\
\hline & & -Useful for sensing \\
\hline
\end{tabular}




\subsection{Methodology Overview}

\subsubsection{Amino Acid Library Creation}

Amino acid libraries used in SELEX are typically displayed on the surface of living microorganisms. Generally, coding regions are inserted into phage, bacteria, or yeast that encode for surface expression of the randomized library. After expression of the library, it is incubated with the target cells of interest. Those that do not bind are discarded while those that do bind are kept and amplified. This amplification is performed by the natural replication of the microorganism. This enriched library is then incubated with the negative target cells. Those molecules that bind these cells are discarded while those that do not are amplified. Between every few rounds, the diversity of the enriched library is assayed by DNA sequencing of the amino acid library-encoding plasmid. The process continues until just one or a few specific and strongly-binding amino acid MREs are present. These are then assayed for their binding, specificity, and stability.

\subsubsection{Phage Display}

Bacteriophage have been used to display amino acid libraries. Libraries of short, random peptides that are displayed on the surface of phage have been used for SELEX experiments and are commercially available (e.g. Phage Display Peptide Library from New England Biolabs; Beverly, MA). These typically consist of 7-15 random peptides displayed by fusion to a surface protein. An example of this is the pIII protein of M13 phage, which is necessary for phage infection of Escherichia coli (Giebel et al. 1995). Random nucleic acid codons are synthesized and inserted into the plasmid for protein fusion. These libraries often consist of approximately $10^{9}$ different peptides. 
Additionally, a single chain Fragment variable (scFv) antibody fragment library has been constructed that is displayed on M13 phage (Pansri et al. 2009). An antibody fragment (Fab) library has also been constructed that is fused to the surface protein D of bacteriophage lambda (Huse et al. 1989). In this case, non-immune human antibody fragments are amplified from donors and constructed into a vector which displays the fusion protein on the virus surface. It has been noted that phage lambda is more capable of displaying large antibody fragments than M13 (Vaccaro et al. 2006).

Phage displayed amino acid libraries offer the advantages of rapid screening and amplification for the SELEX process. Selected phage are incubated with E. coli and plaques are produced. The replicated phage are then subjected to the next round of selection. The method used for SELEX is often panning (see below). While rapid, this method is not as efficient at isolating specific molecules as fluorescent activated cell sorting (FACS) (see below). A careful outline for the use of phage display has been published (Kay et al. 2001). Rapid and efficient amplification coupled with detailed protocols allow phage displayed amino acid libraries to be useful tools in cell SELEX.

\subsubsection{Bacterial Display}

Another platform for amino acid library display is through the use of bacterial hosts. A peptide display library of 12 random amino acids has been displayed as fusions to the $E$. coli flagellin gene (Lu et al. 1995). This library contains approximately 5 X $10^{10}$ different peptides and is available commercially (Invitrogen; Grand Island, NY). A library of the same size consisting of 15 random peptides has been displayed as a fusion to the $E$. coli outer membrane protein A (ompA) (Bessette et al. 2004). These libraries offer the rapid and efficient screening necessary to create a MRE. 
Antibody fragment display has also been performed in bacterial systems. A sub-library of scFv molecules in E. coli was created by PCR randomization of immune antibody fragments (Daugherty et al. 1998). Additionally, scFv molecules have been expressed as fusions to the surface protein A of gram-positive Staphylococcus carnosus (Gunneriusson et al. 1996). These constructs, however, have not been extensively explored for display of entire libraries or their use in SELEX experiments.

Bacterial display of amino acid libraries provides the benefit of rapid screening and amplification. Furthermore, they can be used in both panning and FACS-based separation (Kronqvist et al. 2008). While not as widely used as phage libraries, these libraries do not require multiple hosts, therefore increasing speed of the selection. In addition, detailed protocols for their use are available (Lu et al. 2003). These benefits render bacterial display of amino acid libraries a potential avenue for new developments in developing MREs.

\subsubsection{Yeast display}

Yeast display of amino acid libraries is a widely-used platform for generating MREs. A library consisting of approximately $10^{7}$ random twelve amino acid peptides has been constructed (Krauland et al. 2007). It was formed as a C-terminal fusion to the Aga2p protein that is expressed on the surface of Saccharomyces cerevisiae. This library has proven to be of use in the selection of MREs.

The use of S. cerevisiae to display antibody fragments has also been extensively

explored. This microorganism has been used to display a scFv library of approximately $10^{9}$ different antibody fragments (Boder and Wittrup 1997). This library is freely available from the Pacific Northwest National Laboratory (PNNL; Richland, WA). A Fab sub-library has also been constructed that displays on the surface of yeast (van den Beucken et al. 2003). 
Yeast display of amino acid libraries offers the advantages of use in FACS, as most include sequences for fluorescent protein tagging. Importantly, eukaryotic yeast have the ability to perform post-translational modifications not available in bacteria or phage. This allows for expressing the full diversity of antibody fragment libraries (Feldhaus and Siegel 2004). The described yeast systems also offer easy secretion and purification of selected MREs. Protocols for using these libraries are also easily available (Wang and Shusta 2005; Chao et al. 2006). These attributes allow the use of yeast in protein expression platforms to be an important tool in obtaining MREs by cell SELEX.

\subsubsection{Nucleic Acid Library Creation}

The design of a single stranded nucleic acid library for in vitro selection largely focuses on determining constant regions for PCR amplification. These are often designed by individual laboratories, however both DNA and RNA libraries are available for commercial purchase (e.g. TriLink BioTechnologies; San Diego, CA). A DNA library itself consists of two regions with known bases flanking a region with randomized bases. A RNA library also contains a promoter region to allow for reverse transcription of the library before PCR amplification. The greatest consideration in designing a DNA library is abrogating primer dimer formation and primer secondary structure effects. It is important to determine whether designed primer sequences will bind to each other or themselves (hetero- and homo-dimers), or if their reverse complement sequences will bind to one another, as all four sequences will be in the amplification reaction. Additionally, highly favorable secondary structures of primer sequences may inhibit PCR, and thus needs to be avoided for all four primer sequences. These can easily be interrogated using online PCR primer design software (e.g. Integrated DNA Technologies; Coralville, IA). The size of the randomized region must next be determined. This region must be designed to confer 
the pool sufficient diversity, which can be represented by $4^{\mathrm{n}}$, with $\mathrm{n}$ being the number of random bases in the pool. For example, a pool with 34 random bases will have $4^{34}$, or approximately $2.95 \times 10^{20}$, different possible sequences. Thus, longer random regions contain exponentially greater possible sequence space. In spite of this, longer random regions are more difficult to analyze for consensus binding sequences and are more likely to form secondary structures which inhibit PCR amplification. Therefore, random regions are typically between 20 and 80 bases, making overall libraries shorter than 150 bases (Hall et al. 2009). Longer regions are sometimes necessary, for example in obtaining catalytic MREs (Sabeti et al. 1997).

After design of the library for either DNA or RNA selections, it may be chemically synthesized using phosphoramidite chemistry (Hall et al. 2009). The library must then be purified from remnants of the synthesis reaction, and PCR amplification conditions optimized. During this optimization step, the library may not amplify properly, and it will be necessary to re-design it to suit the researcher's needs. Up to $10^{15}$ different sequences in the library are then incubated with the target of interest to begin the in vitro selection.

\subsubsection{Amino Acid Separation Methods}

A necessity of the SELEX process is separating molecules that bind to the target from those that don't. In cell SELEX, this is typically done by panning, magnetic sorting, or FACS. Often, a combination of these methods is used in order to most efficiently select MREs with high affinity (Table 1.2).

Panning, as used in cell SELEX, is a method of incubating the library with the target cells directly in the tissue culture well or flask. The unbound microorganism-displayed molecules are simply aspirated or decanted. The flask is washed typically three times taking care not to detach the target cells. The bound molecules are then amplified by replication of their host. This 
process is most thoroughly described for phage-displayed libraries (Watters et al. 1997; McGuire et al. 2009) but has also been explored for yeast libraries (Wang and Shusta 2005). For phage, the bound molecules must be eluted and plated on bacteria for replication. In bacterial or yeast libraries, however, the appropriate microbiological media can be added to the cell culture flask or the human cells can be scraped from the flask. The enriched library-containing bacteria or yeast will amplify in this media, while any remaining human cells will not survive under these

Table 1.2. Typical methods of separation in SELEX.

\begin{tabular}{|l|l|l|l|}
\hline Method & Commonly Used With & Advantages & Disadvantages \\
\hline Panning & $\begin{array}{l}\text { Amino acid libraries, some } \\
\text { filter-immobilized targets } \\
\text { with nucleic acid libraries }\end{array}$ & $\begin{array}{l}\text { High throughput, } \\
\text { quick }\end{array}$ & $\begin{array}{l}\text { Less powerful } \\
\text { separation, some } \\
\text { undesired molecules } \\
\text { remain after washes }\end{array}$ \\
\hline $\begin{array}{l}\text { Magnetic } \\
\text { Separation/ } \\
\text { MACS* }\end{array}$ & $\begin{array}{l}\text { Typical for magnetic bead- } \\
\text { immobilized targets with } \\
\text { nucleic acid libraries, MACS } \\
\text { can be used for amino acid } \\
\text { library separation }\end{array}$ & High throughput & $\begin{array}{l}\text { Limited space if } \\
\text { using large cells } \\
\text { (clogging), higher } \\
\text { failure chance } \\
\text { compared to } \\
\text { panning with no } \\
\text { advantages }\end{array}$ \\
\hline FACS* & $\begin{array}{l}\text { Typical for amino acid } \\
\text { libraries }\end{array}$ & $\begin{array}{l}\text { Very powerful } \\
\text { separation ability } \\
\text { with efficient } \\
\text { tunability }\end{array}$ & $\begin{array}{l}\text { Lower throughput, } \\
\text { slower processing, } \\
\text { expensive } \\
\text { equipment }\end{array}$ \\
\hline $\begin{array}{l}\text { Non-magnetic } \\
\text { target } \\
\text { immobilization }\end{array}$ & $\begin{array}{l}\text { Nucleic acid libraries; e.g. } \\
\text { filter columns }\end{array}$ & $\begin{array}{l}\text { Rapid, cheap, high } \\
\text { throughput }\end{array}$ & $\begin{array}{l}\text { Less powerful } \\
\text { separation, may } \\
\text { require many rounds }\end{array}$ \\
\hline $\begin{array}{l}\text { Capillary } \\
\text { Electrophoresis }\end{array}$ & Nucleic acid libraries & $\begin{array}{l}\text { Very powerful } \\
\text { separation ability } \\
\text { with efficient } \\
\text { tunability }\end{array}$ & $\begin{array}{l}\text { Expensive and } \\
\text { expertly-used } \\
\text { equipment }\end{array}$ \\
\hline
\end{tabular}

*MACS is Magnetic-Activated Cell Sorting. FACS is Fluorescence-Activated Cell Sorting.

conditions. Regarding negative selections, the unbound library molecules are removed by careful pipetting. The collected host organisms are then placed under optimal growth conditions. This method of separation is robust for large libraries. However, flocculation of the host 
organisms or inefficient removal of non-binding molecules is possible. This creates an enriched library that contains some molecules that do not bind to the target. Therefore, panning is an efficient method of initial screening for the first few rounds of SELEX.

Magnetic sorting is another mechanism for separating binding for non-binding molecules. Often, magnetic-activated cell sorting (MACS) is used as a pre-enrichment procedure for similar reasons to those for panning. In the case of non-cellular targets such as proteins, the target is typically attached to magnetic beads and the library is passed through the column (Rice et al. 2006). A variation of this used a B cell antigen to bind chronic lymphocytic leukemia cells to magnetic beads for use with a phage library (Takahashi et al. 2003). The library molecules bound to the target are then eluted and replicated under appropriate conditions (or unbound molecules for negative selections). While useful for robust screening, this method may be difficult for use in cell SELEX due limited space within the column when considering human cells and library host cells are present. Therefore this method requires more preparation and greater potential for failure while performing the same pre-enrichment task as panning. It is for these reasons that the use of MACS for cell SELEX in obtaining amino acid MREs is rare in the literature.

Fluorescence-activated cell sorting (FACS) is often used to select MREs that bind to molecules on the surface of cancer cells. This method uses dual fluorescent labeling of the library and the target cells, which are removed from the culture flask. After incubation together, this mixture is subjected to FACS, wherein the software is instructed to collect events corresponding to double fluorescence. For negative targeting, collecting single fluorescent events corresponding to the library alone will render molecules that do not bind to the target. A basic protocol for yeast-displayed scFv library SELEX using FACS has been described 
(Feldhaus et al. 2003). Additionally, a bacterial-displayed peptide library has been used for cell SELEX through the FACS method (Dane et al. 2006). The greatest advantage of FACS is the tunability of separation parameters and extremely efficient enrichment of binding molecules. It does, however, take considerably longer to screen very large numbers of cells. This is exacerbated when using two largely different cell sizes, as is the case with human and bacterial or yeast cells. Also, expensive equipment and expertise in operation is necessary. Therefore, FACS is more efficiently used after the library has been enriched and it is not necessary to use a large number of host cells to ensure full diversity is represented.

\subsubsection{Nucleic Acid Separation Methods}

Just as in selection of amino acid MREs, it is necessary to remove molecules that are not bound to the target of interest. Typically, this involves immobilization of the target of interest onto a substrate. There are separation techniques, such as capillary electrophoresis, that do not require this however (Mosing et al. 2005).

Immobilization of the target of interest is the most common form of nucleic acid MRE selection. This can be done with magnetic beads for manipulation in solution by a magnet, allowing library molecules not bound to the immobilized target to be simply washed away (Stoltenburg et al. 2005). This method has been successful for both protein and small molecule targets (Wang et al. 2009; Kim et al. 2010). Additionally, both protein and small molecule targets have been immobilized onto non-magnetic beads for column chromatography-based selection of MREs (Jackson et al. 2009). Protein targets can also be adsorbed onto nitrocellulose filter paper, however this is more difficult for small molecule targets (Ulrich et al. 1998; Sayer et al. 2002). A variation of this technique is to immobilize the target onto a microfluidic chamber 
for rapid MRE identification (Lou et al. 2009; Ahn et al. 2011). Immobilization of the target typically occurs by conjugation through combinatorial chemistry approaches or adsorption. Other immobilization techniques have been developed, however they all ensure facile separation of bound from unbound molecules.

\subsection{Amino Acid Molecular Recognition Elements for Cell Surface Targets}

\subsubsection{Peptide MREs}

Molecular recognition elements selected from peptide libraries have been extensively explored. Especially from phage display libraries, the cell SELEX procedure has been used to select MREs that bind specifically to tumor cells. Peptides are short sequences generally less than 25 amino acids. Libraries with a very large diversity can be formed drawing from the inherent diversity of the 20 amino acids. A theoretical maximum for a 25 amino acid library is $\sim 10^{32}\left(20^{25}\right)$ or $1.28 \mathrm{X} 10^{9}$ for a seven amino acid library. However, typical construction techniques and reasonable working volumes typically limit library size to around $10^{10}$ molecules. Additionally, there is some bias in insertion of particular amino acids (Krumpe et al. 2006). A breakthrough in peptide library construction was the introduction of codon phosphoramidites (Glen Research; Sterling, VA), which ensures no premature stop codon or insertional biases are present. Compared to antibody fragment libraries, there may be less inherent structure within the MRE, but randomness is ensured.

Peptide MREs in cell SELEX have largely been selected through phage display methods. An example of this is the selection of peptide MREs that bound to the B-cell lymphoma line A20 (McGuire et al. 2006). This experiment did not use differential cell SELEX, but they were able to differentiate lymphoma cells from other normal white blood cells. In another study, primary chronic lymphocytic leukemia (CLL) cells were the targets of phage selection using a modified 
MACS protocol (Takahashi et al. 2003). This study also did not use negative cell lines, however a panel of peptide MREs were selected that bound to different types of blood cells. A study performed that selected peptide MREs for non-small cell lung cancer (NSCLC) cell lines also did not use a negative target cell (Chang et al. 2009). One of the selected peptides, however, was successfully used for in vivo chemotherapeutic delivery studies. It is therefore possible to select useful peptides without a differential component of the selection.

The likelihood of selecting one or a few specific MREs that are fit for their expected use is increased if negative targets are used. One study selected for peptides that bound to the breast cancer cell line BT-474 but not the benign cell line MCF 10A (Shukla and Krag 2010). This study even did a negative selection against the tissue culture flask itself. This, however, seems to be inherent if negative selections are performed in the same type of flask as positive selections. The selected peptides did not bind to any of four benign cell lines that were used for binding studies. They bound to some, but not all, tumor cell lines studied and internalized into some. Another study with breast cancer cells selected for peptides that bound to HER positive SKBR-3 cancer cells but not HER negative MCF-10A benign cells (Abbineni et al. 2010). The selected peptides showed homology to other ligands for HER2, however the actual cell surface target molecule was not determined. Other studies targeting a follicular thyroid carcinoma cell line (Zitzmann et al. 2007) and separate colorectal tumor cell lines (Rasmussen et al. 2002; Kelly and Jones 2003) have been performed. Separate studies using the metastatic prostate cancer cell lines LNCaP (androgen sensitive) and PC-3 (androgen insensitive) selected peptides that showed no real homology between the reported sequence sets (Romanov et al. 2001; Jayanna et al. 2010). As these peptide sequence families did not converge, it can be concluded that they were not binding to the same cell surface molecule. Therefore it is likely these could be used together 
to differentiate the two forms of prostate cancer and potentially define cell surface molecules present in one but not the other.

There also have been variations of the phage display peptide MRE selection process. A bacterial peptide display library has been used to select molecules that bound to breast adenocarcinoma cells ZR-75-1 but not benign cells (Dane et al. 2006). A variation on phagedisplayed peptide panning has been performed in a microfluidic system for semi-automated selection of MREs (Wang et al. 2011). The above examples of using peptide libraries for selecting MREs express their potential in quick and efficient screening of random molecules to obtain diagnostically or therapeutically useful tools.

\subsubsection{Antibody Fragment MREs}

Antibody fragment MREs have also been selected that specifically bind to tumor cells. Antibody fragment libraries can be in the form of scFv, Fab, or others. These libraries typically have diversity on the order of $10^{9}-10^{10}$ molecules. This well exceeds the natural potential diversity of the human immune system, which has a maximum of approximately $10^{7}$ different antibodies (based on heavy and light chain combinations of genetic diversity). Therefore, antibody-like MREs can be selected from libraries with much greater diversity than in vivo antibody production. It is likely that strong and specifically binding antibody fragments will be selected for cancer cells using differential cell SELEX.

Multiple studies have selected MREs that bind to tumor cells but not normal cells or other types of tumor cells. Two separate studies selected scFv MREs that bind to androgen insensitive prostate cancer cell lines. One targeted PC-3 cells and did negative selections with normal prostate cell lines (Liu et al. 2004). The other targeted the C4-2B cell line, which is derived from LNCaP cells that were used as a negative target (Conrad et al. 2009). The selected 
antibody fragments were used for separate purposes, but the differences in selection design make it unlikely the MREs bind to the same surface molecule. Other studies have selected scFv MREs specific for melanoma cells (Kupsch et al. 1999), hepatocarcinoma (Yu et al. 2005), and ex vivo breast cancer tissue (Jakobsen et al. 2007). Additionally, Fab MREs have been selected that bind to T-cell malignancies (Willemsen et al. 2008) and ovarian carcinoma cells (Figini et al. 1998).

Antibody fragment MREs offer the specificity and pharmacological characteristics of monoclonal antibodies. Additionally however, they offer the selectivity and relative ease in production provided by the differential cell SELEX process. Their multiple potential uses therefore make their selection a strong method for cancer cell studies using SELEX.

\subsection{Small Molecule Nucleic Acid Molecular Recognition Elements}

\subsubsection{DNA MRES}

Molecular recognition elements in the form of DNA have been obtained for a wide variety of targets. These include ions of lead, mercury, and arsenic, among others (Kim et al. 2009; Chung et al. 2012). They also include protein targets such as vasopressin, protein kinase C, VEGF, and many others (Williams et al. 1997; Mallikaratchy et al. 2006; Hasegawa et al. 2008). A variation of this includes the selection of DNAzymes, which are selected to have enzymatic activity (Zhu et al. 2012). Another is the selection of double-stranded DNA MREs that bind to transcription factors (Sooter and Ellington 2004).

There have been a large number of MREs selected for small molecule targets for a variety of purposes. These include the identification of molecules such as ATP within cells (Huizenga and Szostak 1995), detection of pharmaceutical or illicit drugs such as theophylline and cocaine in biological contexts (Liu and Lu 2005; Chavez et al. 2010). DNA MREs have also been selected for environmental monitoring and contaminant detection purposes. Examples 
of this include identifying MREs for pesticides such as acetamiprid or a variety of organophosphates (He et al. 2011; Wang et al. 2012). It has been shown that small molecules are specifically able to intercalate between bases of a DNA MRE in a sequence-dependent manner (Lin and Patel 1997). Additionally, normal antibody production against small molecule haptens require use of an immunogenic carrier, whereas in vitro selection does not, thus increasing specificity of the final binding element (Askonas et al. 1970).

DNA MREs offer a simplified and straightforward selection protocol, as discussed before. Additionally, they are stable as compared to RNA MREs, plus regenerative ability compared to antibodies. They are also very useful for molecular detection, as many references utilize them in this manner. Therefore, selection of DNA MREs offers an ideal molecular detector for detecting small molecules in complex environments.

\subsubsection{RNA MREs}

RNA MREs were historically the first to be identified (Ellington and Szostak 1990; Tuerk and Gold 1990). These molecules were originally identified to bind to protein targets, and many more examples have since been reported. There are also examples of the selection of ribozymes, which have catalytic activity often reliant on heavy metals (Wilson and Szostak 1999; Robertson et al. 2004).

There have also been numerous examples of RNA MREs for small molecule targets (Codrea et al. 2010). Similar to DNA, there have also been RNA MREs selected for ATP (Dieckmann et al. 1996). Additional examples include antibiotics such as chloramphenicol, tetracycline, and kanamycin, among many other types of targets (Burke et al. 1997; Berens et al. 2001; Goertz et al. 2004). Small molecule binding to RNA MREs is likely to occur similarly to DNA MREs. 
RNA MREs typically have a high affinity and specificity for their target of interest. Additionally, these molecules offer many of the same molecular detection applications that DNA MREs do. They are, however, less straightforward to select and more likely to be degraded if used to detect their target in complex matrices. There are examples of modifications at their 2' hydroxyl which lower their degradation, however this often negates their advantage of having a higher binding affinity (Cui et al. 2004; de-los-Santos-Alvarez et al. 2007). It is therefore more straightforward with a higher probability of successful detector integration to select DNA MREs than RNA MREs.

\subsection{Molecular Recognition Element Applications}

\subsubsection{Amino Acid Molecular Recognition Element Applications}

\subsubsection{Therapeutics}

Amino acids in the form of monoclonal antibodies have been widely used as therapeutics (Brekke and Sandlie 2003). Well-known examples include trastuzumab (Herceptin ${ }^{\mathrm{TM}}$ : Genentech Inc.; San Francisco, CA) for HER2 positive breast cancer treatment and bevacizumab (Avastin ${ }^{\mathrm{TM}}$ : Genentech Inc) that binds to vascular endothelial growth factor (VEGF). These examples, among others, show the potential of amino acid-based therapeutic intervention. Peptide therapeutics have been developed and used for a variety of diseases. Examples of these are to inhibit infection (Oyston et al. 2009) and inflammatory diseases (Vally et al. 2006). A review of therapeutic peptides obtained by phage display selection is available (Ladner et al. 2004). Examples of commercial peptide therapeutics include Humulin ${ }^{\mathrm{TM}}$ (Lilly; Indianapolis, IN) for diabetes treatment and leuprolide (Lupron ${ }^{\mathrm{TM}}$ : Abbott Laboratories; Abbott Park, IL) for metastatic prostate cancer treatment. Peptides obtained by cell SELEX are therefore possible alternatives to antibodies for cancer treatment. 
Antibody fragments have also been used therapeutically. As of 2009, approximately 90 scFvs had been patented in the U.S., with about 30 being specifically for cancer therapeutics (Pucca et al. 2011). The humanized h5G1.1-scFv specific for complement protein C5 (pexelizumab: Alexion Pharmaceuticals; Cheshire, CT) has undergone clinical trials for its ability to reduce myocardial infarction after coronary artery bypass surgery (Smith et al. 2011). This molecule was not selected by SELEX, but was engineered from a previously-selected monoclonal antibody (Evans et al. 1995). The results of clinical trials are mixed (Lin 2011), however the pharmacological profile of the antibody fragments does appear to be safe (Fitch et al. 1999). It is just one of 19 scFvs to reach clinical development as of 2010 (Nelson 2010). There have also been 22 Fabs to reach clinical trials, 3 of which have been approved (Nelson 2010). While some of these antibody fragments were engineered from monoclonal antibodies, it is clear that scFv treatments obtained from SELEX will be useful and more specific for cancer treatment.

Another scFv molecule that binds to the HER2 receptor on the surface of tumor cells has undergone clinical trials. The antibody fragment was produced similar to the previous one, however it was conjugated to exotoxin A (scFv-ETA) (Wels et al. 1992). The results of the trials proved safe, and the drug was moderately effective (von Minckwitz et al. 2005). This use of antibody fragments in tumor cell treatments shows the potential for specific drug delivery of various therapeutics.

The use of amino acid MREs is clearly a potentially fruitful field. The experimental tunability for selectivity and in vivo stability has been previously demonstrated. Recently, theranostics, or the dual use of single molecules for therapeutic and diagnostic purposes, has become popular (Goldenberg et al. 1990). There is clearly the possibility of using amino acid 
MREs that have been conjugated to contrast materials as in the previous study. The use of MREs obtained by cell SELEX in therapeutics and/or diagnostics has only begun to be explored but represents great potential.

\subsubsection{Disease Detection}

Amino acid MREs for detection of disease is a potential clinical application of these molecules. For biomarker-based cancer detection, blood is typically extracted and subjected to immunoassays with monoclonal antibodies. There also lies the potential for magnetic, radio-, or fluorescent labeling of these antibodies for diagnostics. Antibodies used in these assays do not always bind to molecules that represent disease states. For example, anti-PSA antibody used in prostate cancer detection has a false-positive rate over 75\% (Thompson et al. 2006). These uses make it important to obtain molecules that specifically detect malignant cells.

Initial research using peptide MREs obtained by SELEX have been promising. Ex vivo human and in vivo mice studies using colon cancer-specific peptides have been performed (Kelly et al. 2004). Using radiolabeled and fluorescent peptides, favorable pharmacological profiles and detection were observed. Similar results were found using an anti-prostate cancer peptide selected by phage display (Zitzmann et al. 2005). While not extensively explored for clinical use, peptide MREs may have great diagnostic potential.

Similar studies to those performed with peptide MREs have been done with antibody fragments (Goldenberg et al. 1990). Another potential use of antibody MREs is in ex vivo diagnostics such as immunoassays or microfluidic cell capture. Though using a monoclonal antibody, previous studies have shown the ability to capture circulating tumor cells with antibodies in a microfluidic device (Goldenberg et al. 1990). There is the obvious potential for 
application of antibody fragment MREs to a similar device. These and other uses make the diagnostic potential of amino acid MREs extremely important.

\subsubsection{Surface Molecule Elucidation and Discovery}

Whether the selected MRE is nucleic or amino acid, it can be used to learn more information about its target cell. An important attribute of cell SELEX is that no a priori knowledge of the cell surface molecular target is necessary. It is also likely that cells in disease states will express surface molecules differently than normal cells. No previous knowledge of this differential expression is necessary however, because the differential cell SELEX process will take advantage of this fact in selecting MREs.

The cell SELEX process has been used to select MREs which aided in identification of their own surface ligand. DNA MREs have been selected that bound to tenascin C overexpressing cells and not unaltered cells (Daniels et al. 2003). As predicted, the MREs bound to tenascin C. Another study determined that ICAM-1, an intercellular adhesion molecule, was the target of scFv MREs selected on androgen-insensitive prostate cancer cells (Conrad et al. 2009). DNA MREs have also been used to discover new proteins expressed on the surface of dendritic cells (Berezovski et al. 2008). These works show that overexpression of a surface protein does allow MRE selection against them. Additionally, novel or confirmatory roles of previously described molecules can be determined. There also lies the potential for discovery of new cell surface molecules or biomarkers. This use of MREs is potentially very important for a fundamental understanding of disease proteomics.

\subsubsection{Nucleic Acid Molecular Recognition Element Applications}

Nucleic acid MREs have great potential in multiple settings. Predominant among these is analyte binding in biosensors. Both DNA and RNA MREs have been incorporated into sensors 
(Cho et al. 2009). Typically, these come in the form of optical, electrochemical, or mass sensitive sensors, sometimes with the incorporation of various nanoparticles for signal transduction (Song et al. 2008). As noted previously, nucleic acid MREs function well in this setting because they can be obtained for most targets, can be modified and incorporated into devices, are relatively stable, often change shape upon ligand binding, and are reusable.

Nucleic acid MRE-based sensors have been developed utilizing many different modalities. For example, sensors for just ATP have been developed using DNA MREs integrated into many types of sensors, including those utilizing field-effect transistors (Goda and Miyahara 2012), fluorescent detection (Urata et al. 2007; Wang et al. 2008), quantum dot luminescence (Huang et al. 2010), surface-enhanced Raman scattering (Li et al. 2012), carbon nanotubes (Zhang et al. 2010), microbalances (Ozalp 2011), electrochemical transducers (Zuo et al. 2009), and many others. These encompass the three main detection modalities mentioned above, and give just a small swath of the large spectrum of methods possible with nucleic acid MREs.

In addition to these many detection methods, MRE-based sensors have been developed for many different targets. These include small molecules such as ATP as noted above, cocaine (Zhou et al. 2012), tetracycline (Kim et al. 2010), and many others. They also include proteins such as thrombin (Cho et al. 2008) and anthrax protective antigen (Cella et al. 2010), and insulin (Yoshida et al. 2009), among others. It is noteworthy that many of these detection mechanisms are modular in that a separate MRE can be used within the same design to detect new analytes.

In addition to detection, nucleic acid MREs have been investigated for various biomedical applications. As noted earlier, Macugen ${ }^{\mathrm{TM}}$ (pegaptanib) is in the clinic for macular degeneration treatment. Other MREs have been used for in vivo therapeutic delivery of drug- 
loaded particles (Wu et al. 2010). They can be integrated into the membrane of lipid-based particles, or immobilized onto the surface of certain manufactured nanoparticles, each having a drug-release mechanism (Yu et al. 2011; Li et al. 2012; Sundaram et al. 2012). Furthermore, they have been complexed with positron emitters, fluorophores, radiolabels, or other transducing elements for in vivo imaging of molecular expression (Tavitian et al. 2009; Shi et al. 2010; Yu et al. 2011). In spite of these applications, they have not been as widely moved into clinical settings as antibodies, largely due to drawbacks previously discussed.

In total, nucleic acid MREs have a wide array of potential applications. They have been studied for their biomedical uses; however their properties allow them to be more useful in biosensors. Thus, nucleic acids MREs will be the focus of molecular detection for sensing applications.

\subsection{Conclusions}

Selection of MREs using differential cell SELEX represents a powerful method of differentiating between cell types. This process requires no prior knowledge of cell surface molecule expression and takes advantage of differential expression profiles. Peptide and antibody fragment libraries, the host on which the library is displayed, and the selection method each have advantages and situations in which they are useful. Expansion of host scaffolds has been performed, even using cereal crops (Stoger et al. 2000) and human HEK-293T cells (Ho and Pastan 2009) to display engineered scFv molecules. As more MREs are being developed, it is certain that their uses and clinical investigation will continue to expand.

Nucleic acid MREs have a many ideal applications, and are able to be obtained for a wide array of targets. They yield a powerful method of molecular binding and differentiation of molecules. As discussed, they are particularly useful in biosensors for detection of various 
analytes. Increasingly more detection modalities are being developed and are only limited by creativity of their integration. Therefore, the continued selection and investigation of nucleic acid MREs is important for a variety of applications.

Therefore, the work discussed herein developed different types of MREs for different applications. In total, the SELEX process was applied to obtaining MREs for three different targets. Specifically, this work developed and utilized a novel variation called Decoy-SELEX which focuses on what the final MRE should not bind to. As in classical SELEX, the library is enriched for the target of interest, however many stringent negative selections are performed. Antibody fragment MREs were obtained that could bind to prostate cancer cells and differentiate between them and benign prostatic cells. These have potential in surface molecule discovery, cancer detection, and targeted therapy. Separately, DNA MREs were obtained for two small molecule pesticides, atrazine and malathion. These have potential use as binding molecules in biosensors for these environmental contaminants. In total, each MRE has strong affinity and discriminatory power, as well as multiple potential applications building upon this work. 


\section{CHAPTER 2}

In Vitro Selection of Single-Stranded DNA Molecular Recognition Elements for the Herbicide Atrazine 


\subsection{Introduction}

Atrazine is a chlorotriazine herbicide used widely in crop production. An estimated 76.5 million pounds are used per year in the United States alone (USGS 2002). Its use is estimated to increase crop yields 4-6\% (Ackerman 2007). The economic benefits of atrazine use have led to its continued application for many agricultural processes.

The widespread application of atrazine, however, leads to contamination of aquatic ecosystems and water supplies. Dependent upon rainfall, up to $20 \%$ of atrazine applied to crops may run off or be leached from soils (Graymore M 2001). Streams bordering croplands applying atrazine have been shown to be contaminated at levels up to $1000 \mu \mathrm{g} / \mathrm{L}$. Additionally, some drinking water reservoirs in the U.S. have up to $88.4 \mu \mathrm{g} / \mathrm{L}$. Atrazine has been detected in surface and ground waters in the state of West Virginia (Pasquarell and Boyer 1996; Ator and Ferrari 1997). Removal of atrazine in water treatment plants is possible, but varies widely throughout the US (Jiang H 2006). The US Environmental Protection Agency (EPA) Maximum Contaminant Level (MCL) is $3 \mu \mathrm{g} / \mathrm{L}$ for human exposure to atrazine (USEPA 2011). This level has been exceeded in many surface waters tested in West Virginia (Ferrari and Assessment 1997). Furthermore, the European Union has banned use of atrazine due to contamination levels above recommended limits (Sass and Colangelo 2006).

The recommended limit of exposure is based on the potential negative effects of atrazine. Atrazine exposure at environmentally relevant levels affects aquatic organisms; including feminization of male frogs (Hayes et al. 2002), salamander desiccation (Rohr and Palmer 2009), and endocrine disruption in fish (Suzawa and Ingraham 2008). Endocrine disruption is also a potential mechanism of atrazine action in humans and other mammals (Cooper et al. 2007; Fan et al. 2007; Albanito et al. 2008). It has been shown to interact with a steroidogenic transcription 
factor and a growth factor receptor, causing signaling pathways to be upregulated. This may lead to hormonally-linked cancers and reproductive abnormalities. Additionally, atrazine may affect innate immune function and increase damage caused by other toxins such as arsenic (Tchounwou et al. 2001; Mizota and Ueda 2006). There is, however, evidence that contradicts an EPA finding that atrazine is not a potential carcinogen (Mills 1998). It is therefore important that food and water supplies be routinely monitored for atrazine levels.

The current methods of atrazine detection are either time- and labor-intensive or are not specific for the herbicide. The most common method is gas chromatography, which requires extensive sample preparation (Amistadi et al. 1997). Combinatory chromatographic methods have been used to determine the presence of atrazine in both serum and urine (Brzezicki et al. 2003; Panuwet et al. 2008). Antibody-based assays are also used, however the antibody used cannot differentiate between atrazine and closely-related molecules (Wortberg et al. 1996; Suri et al. 2009). It is therefore necessary to identify an easily-synthesized binding element with high affinity and selectivity. Utilizing Decoy-SELEX (Systematic Evolution of Ligands by Exponential Enrichment) an atrazine-specific Molecular Recognition Element (MRE) was identified with sub-nanomolar affinity.

Molecular Recognition Elements are antibody-like molecules that strongly and selectively bind to a target of interest. They are produced by the in vitro selection process initially described independently by the laboratories of Gold and Szostak in 1990 (Ellington and Szostak 1990; Tuerk and Gold 1990). MREs are selected from a large $\left(10^{9}-10^{15}\right)$ library of single-stranded DNA or RNA. The library is initially incubated with the target of interest. Molecules that do not bind to the target are discarded and those that do bind are amplified. These are then subjected to a series of negative targets, after which molecules that do not bind 
are amplified. This iterative process continues, utilizing increasingly stringent conditions, until a MRE that specifically binds to the target of interest, but not negative targets, is identified. The selected MRE may then be used for applications such as detection of the target molecule.

A single-stranded DNA (ssDNA) MRE with high affinity and specificity for atrazine was isolated using a novel Decoy-SELEX process. This SELEX variation enriches the library for the target of selection but focuses on stringent negative selections against undesirable binding targets. Those negative targets are chosen by chemical similarities, such as target metabolites, or their environmental proximity to which they are found with the target. The selection scheme for atrazine was designed to ensure the MRE would bind to neither atrazine metabolites diaminochloro-triazine (DACT) or desethyl atrazine nor the closely-related herbicide simazine (Muller et al. 1997). It was also designed so that the MRE would not bind to propanil, 2,4-D acid, or malathion, which are pesticides found at similar levels and environments to atrazine (Kucklick and Bidleman 1994; Coupe et al. 2000; Purcell et al. 2001; McCarthy and Fuiman 2008). These selected molecules may then be incorporated into a sensing device, allowing for specific atrazine detection (e.g. (Soh et al. 2009; Yang et al. 2010; Lin et al. 2011)).

\subsection{Methods}

\subsubsection{Selection of Atrazine-Specific MREs}

In order to select an atrazine-specific MRE, the Decoy- SELEX process was used (Figure 2.1). A single stranded DNA (ssDNA) library consisting of $10^{15}$ different molecules was screened through 12 rounds of selection. The ssDNA library, termed RMW.N34, consists of two 23 base constant priming regions for polymerase chain reaction (PCR) amplification flanking a 34 base random region (synthesized by Eurofins MWG Operon; Huntsville, AL). From this library, molecules that have a sequence and tertiary structure that specifically binds to atrazine 
were selected. Molecules in the library which bound to closely-related structures and other pesticides were removed (Figure 2.2).

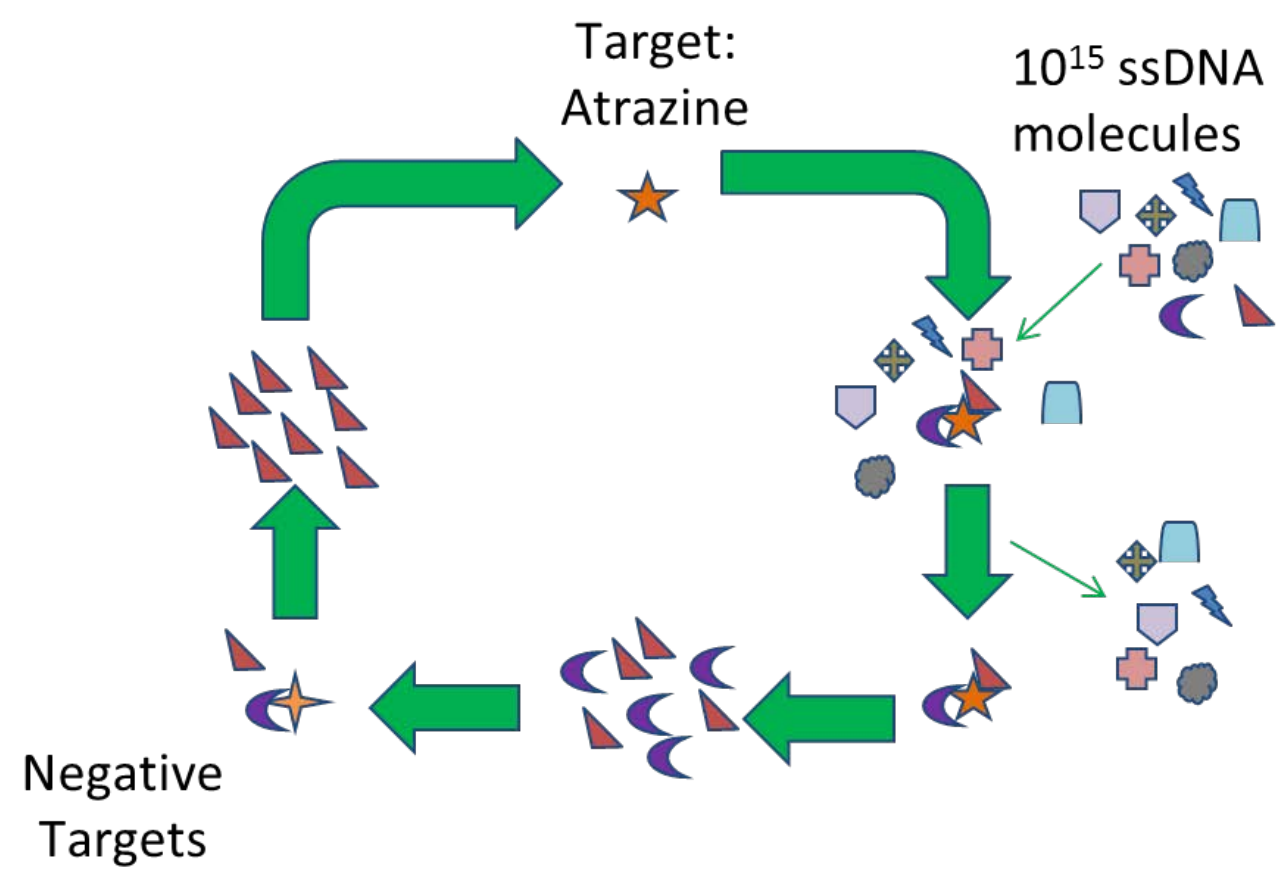

Figure 2.1. Scheme depicting the Decoy-SELEX process for atrazine MRE selection. A library of $10^{15}$ ssDNA molecules were incubated with the target atrazine. Those that bound were amplified and incubated with negative targets. Those that did not bind the negative targets were amplified and subjected to additional rounds of in vitro selection.

An atrazine derivative, desethyl atrazine (Figure 2b) (Fluka Analytical; St Louis, MO) was covalently biotinylated by Sulfo-NHS-LC-Biotin (Pierce; Rockford, IL) per manufacturer's instructions. Biotinylated desethyl atrazine was then incubated with streptavidin-coated magnetic beads (New England Biolabs; Ipswich, MA) to immobilize the selection target. Unbound desethyl atrazine was washed to leave only biotinylated desethyl atrazine bound to magnetic beads (immobilized target).

For positive immobilized target selection, the ssDNA library was incubated with $50 \mu \mathrm{L}$ immobilized target in $500 \mu \mathrm{L}$ of buffer consisting of $100 \mathrm{mM}$ sodium chloride, $20 \mathrm{mM}$ Tris-HCl, and $2 \mathrm{mM}$ magnesium chloride (1X selection buffer; SB). After an incubation period with 
rotation at room temperature, the immobilized target was separated from the solution by magnet, washed three times with $1 \mathrm{~mL} \mathrm{SB}$, and resuspended in SB. This suspension served as a template for PCR amplification of bound ssDNA. PCR conditions were as follows: enriched ssDNA library, $400 \mathrm{nM}$ forward and biotinylated reverse RMW.N34 primers (Eurofins MWG Operon; Huntsville, AL) (forward: 5'-TGTACCGTCTGAGCGATTCGTAC-3', biotinylated reverse: 5'Biotin- GCACTCCTTAACACTGACTGGCT -3'), $250 \mu$ M deoxynucleotide triphosphates, $1 \mathrm{X}$ GoTaq Reaction Buffer (Promega; Madison, WI), 3.5 units Taq, and pure water. Reaction conditions were: initial denaturation of $95^{\circ} \mathrm{C}$ for 5 minutes; cycling at $95^{\circ} \mathrm{C}$ for 1 minute, $63^{\circ} \mathrm{C}$ for 45 seconds, and $72^{\circ} \mathrm{C}$ for 1 minute; and final extension of $72^{\circ} \mathrm{C}$ for 7 minutes. Large-scale amplification of $4 \mathrm{~mL}$ and $2 \mathrm{~mL}$ was done preceding positive and negative selection rounds, respectively. This selection scheme for the desethyl atrazine target occurred for Rounds 1-5 of selection, each time with a shorter incubation period.

Amplified dsDNA was then purified via the GFX PCR purification kit (GE Healthcare; Piscataway, NJ). Eluted dsDNA from the column was incubated with streptavidin agarose resin (Pierce; Rockford, IL). Having used a biotinylated primer for the reverse strand amplification allowed for binding of the amplified dsDNA to the agarose resin. This was placed into a flowthrough column and washed with 5 volumes of 1 X PBS. Five volumes of $1 \mathrm{M}$ sodium hydroxide were then used to dissociate the DNA strands, eluting the forward strand ssDNA from the column. This solution was neutralized with 0.1 volume $3 \mathrm{M}$ sodium acetate ( $\mathrm{pH}$ 5.2), to which 2.5 volumes of cold $100 \%$ ethanol were added. Glycogen at $10 \mu \mathrm{g} / \mathrm{mL}$ was added as a coprecipitate for ethanol precipitation, and the solution was frozen. After freezing, this was centrifuged at 13,000xg for 1 hour, washed with 70\% ethanol, and centrifuged again for 15 


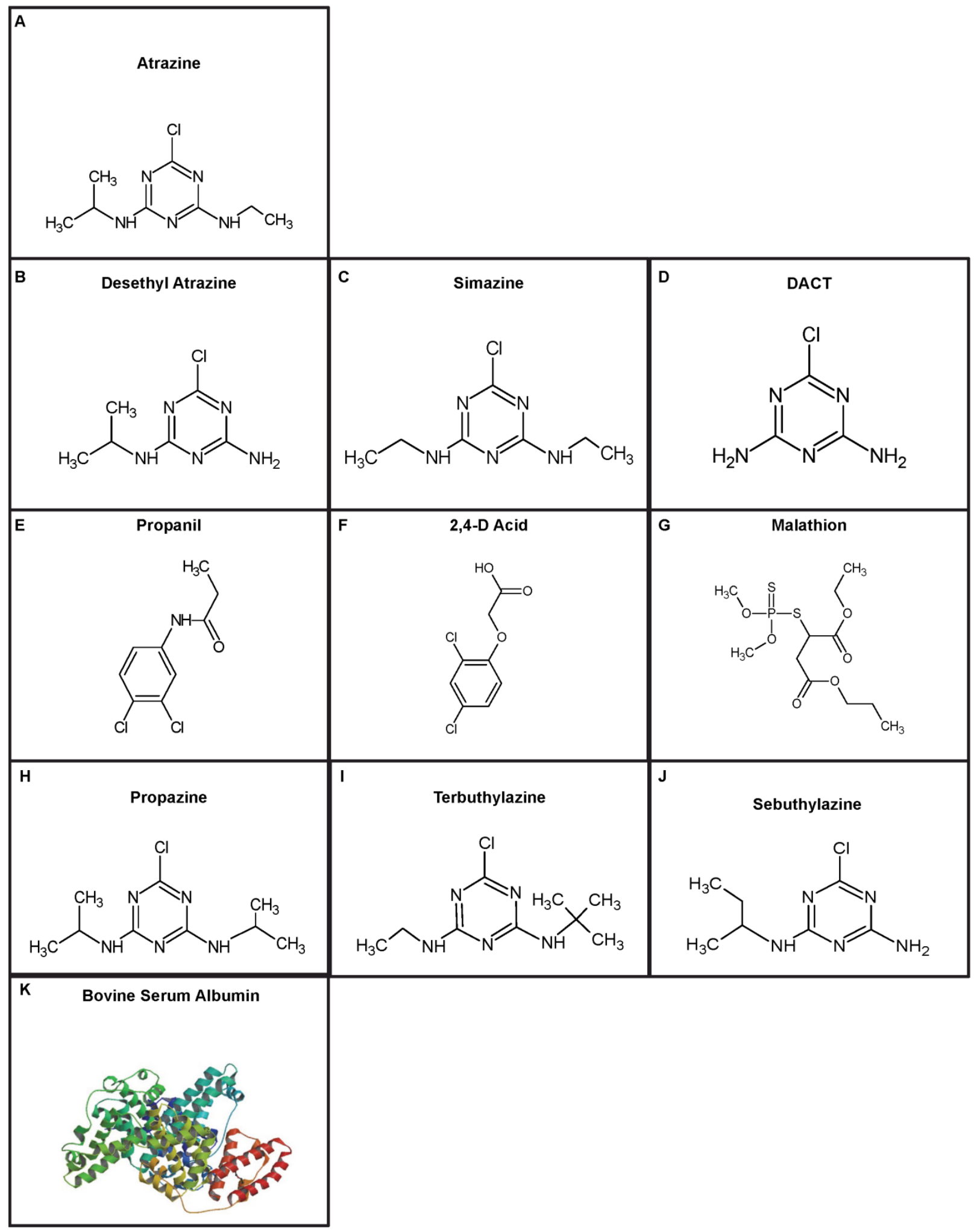

Figure 2.2. Chemical structures of molecules used in the Decoy-SELEX scheme and crossbinding assays. (a) Structure of the herbicide and target of selection atrazine. (b) Structure of atrazine derivative desethyl atrazine, used to immobilize onto magnetic beads. (c) and (d) 
Structures of simazine and diamino-chloro-triazine (DACT): chemically similar to atrazine, and used in rounds of negative selection. (e), (f), and (g) Structures of propanil, 2,4-D acid, and Malathion: pesticides found in ecologically relevant environments to atrazine and used in negative selections. (h), (i), and (j) Structures of propazine, terbuthylazine, and sebuthylazine: structurally similar metabolites of atrazine used in cross-binding assays. (k) Ribbon structure of bovine serum albumin (BSA) used in cross-binding assay (Bujacz 2012).

minutes. The supernatant was then decanted and the ssDNA pellet dried under a vacuum. The purified ssDNA pellet was resuspended in SB and subjected to another selection incubation.

Negative selections were done to ensure the ssDNA MRE is specific for atrazine. SulfoNHS-LC-Biotin (Pierce; Rockford, IL) was blocked with Tris-HCl. Then the blocked biotin reagent was incubated with streptavidin coated magnetic beads and washed with SB (immobilization substrate). $50 \mu \mathrm{L}$ of blocked biotin reagent immobilized onto magnetic beads was incubated with purified ssDNA from the positive selection in a $100 \mu \mathrm{L}$ volume. After an incubation period, the supernatant was removed and beads were washed three times with 25,25 , and $50 \mu \mathrm{L}$ SB, with removed supernatant and washes serving as a PCR template. Amplified dsDNA was then prepared for another selection round as outlined above. Negative selections done in this manner occurred in Rounds 1, 3, 5, and 11.

Round 6 of selection was done as stated above, however the selection buffer consisted of $10 \%$ methanol in $1 \mathrm{X}$ selection buffer (methanol selection buffer; MSB) to ensure ssDNA binding in ensuing selection rounds. For Rounds 7-11 of positive selection, the purified ssDNA library was incubated with immobilized target as outlined above. However, after three washes, $100 \mu \mathrm{L}$ of $1 \mathrm{mM}$ atrazine in MSB was incubated with the beads for decreasing time periods. The ssDNA bound to the immobilized target was separated from the ssDNA in the solution phase and served as a template for PCR amplification. Preparation of the library for the ensuing round of selection was done as noted above. 
The Round 8 negative selection was done as described above, however ssDNA was competitively eluted with the pesticides propanil, 2,4-D acid, and malathion (Figure 2.2 e-g). The ssDNA bound to the immobilized target was separated from solution and resuspended, serving as the template for PCR amplification. Rounds 9 and 10 of negative selection were done in the same manner, with DACT and simazine (Figure $2.2 \mathrm{c}-\mathrm{d}$ ) serving as targets for competitive elution, respectively.

The final round of positive selection, Round 12, was done similar to the competitive elutions above. However, bound ssDNA was eluted with $100 \mu \mathrm{L}$ of $100 \mu \mathrm{M}$ atrazine in MSB. This eluted ssDNA served as a template for PCR amplification.

\subsubsection{Sequencing of Atrazine-Specific MREs}

Every third round of selection, the ssDNA library was analyzed for consensus binding sequences by DNA sequencing. The PCR-amplified (using non-biotinylated primers) library was ligated into the pCRII vector (Invitrogen, Carlsbad, CA) and cloned into competent bacteria per manufacturer's instructions. The plasmid was then extracted using the AxyPrep Plasmid Prep Kit (Axygen, Union City, CA) and sent for sequencing (Eurofins MWG Operon, Huntsville, AL) using the M13R primer complementary to a region upstream of the PCR insert in the pCRII vector. Analyses were done on 30-50 random sequences for each round to identify consensus sequence binding families.

\subsubsection{Atrazine MRE Binding Assays}

From the Round 12 sequences, three sequences were chosen for further characterization. The predicted tertiary structures were obtained using the Mfold DNA web server at $25^{\circ} \mathrm{C}$ with buffer salt conditions (SantaLucia 1998; Peyret 2000; Zuker 2003). R12.23, R12.28, and R12.57 
were chosen and synthesized by Eurofins MWG Operon with an AlexaFluor 488 label for use in binding studies.

Concentrations of $0,0.25,0.5,0.75,1,5,10,15,20$, and 25 nM MRE were used in saturation binding studies. In a $200 \mu \mathrm{L}$ total volume of MSB, $10 \mathrm{uL}$ of immobilized target was incubated with each concentration for five minutes. Unbound MRE was removed and each incubation was washed five times with $200 \mu \mathrm{L}$ MSB, then the immobilized target was resuspended in $200 \mu \mathrm{L}$ MSB and heated to $95^{\circ} \mathrm{C}$ for 10 minutes to denature and elute bound MRE. Eluted ssDNA in MSB was placed in a 96-well microplate and measured in a Synergy 2 microplate reader equipped with a tungsten halogen lamp with excitation wavelength of $485 \mathrm{~nm}$ and an emission filter at $520 \mathrm{~nm}$ using Gen5 1.06 software (Biotek US; Winooski, VT). All fluorescence readings on the plate were normalized to $100 \mu \mathrm{L}$ of a $1 \mathrm{nM}$ solution in SB of the fluorescent MRE and the no ssDNA incubation. For each concentration set, the same was done with washed streptavidin-coated magnetic beads to ensure binding over background to the beads. Each set of incubations were performed in triplicate. To determine the dissociation constant of R12.23, data were analyzed with Origin 8 (OriginLab Corporation; Northampton, MA) using nonlinear regression analysis and fit with the equation, $\mathrm{Y}=\left(\left(\mathrm{B}_{\max } * \mathrm{X}\right) /\left(\mathrm{K}_{\mathrm{d}}+\mathrm{X}\right)\right)+\mathrm{NS} * \mathrm{X}$, where $\mathrm{B}_{\max }$ is the maximum binding, $\mathrm{K}_{\mathrm{d}}$ is the dissociation constant, and NS is nonspecific binding as previously described (Langan et al. 2012).

To determine binding of the selected MRE, R12.23, to negative targets used in the selection, $5 \mathrm{nM}$ fluorescent ssDNA was used. For each elutant used, in a $200 \mu \mathrm{L}$ total volume of MSB, $10 \mu \mathrm{L}$ of immobilized target was incubated with R12.23 for five minutes. The magnetic beads were washed five times with $200 \mu \mathrm{L}$ MSB. Then, each incubation was resuspended in 200 
$\mu \mathrm{L}$ of $100 \mu \mathrm{M}$ of the following in MSB: atrazine, desethyl atrazine, DACT, simazine, propanil,

2-4, D Acid, malathion, and MSB only. An incubation with no R12.23 present and

Table 2.1. Decoy-SELEX scheme for atrazine MRE selection.

\begin{tabular}{|l|l|l|}
\hline Round & Positive Selection & Negative Selection \\
\hline 1 & Immobilized Target (IT) 25 hrs & Immobilization Substrate (IS) 23 hrs \\
\hline 2 & IT 23 hrs & - \\
\hline 3 & IT 18 hrs & IS 18 hrs \\
\hline 4 & IT 12 hrs & - \\
\hline 5 & IT 10 hrs & IS 16 hrs \\
\hline 6 & IT w/ methanol buffer 12 hrs & - \\
\hline 7 & $\begin{array}{l}\text { IT } 10 \text { hrs, Competitive Elution } \\
\text { w/ } 1 \text { mM Atrazine (CE) } 5 \text { min }\end{array}$ & - \\
\hline 8 & IT 2 hrs, CE 2 min & $\begin{array}{l}\text { IT } 2 \text { hrs, CE w/ propanil, 2,4-D acid } \\
\text { malathion, } 5 \text { min }\end{array}$ \\
\hline 9 & IT 5 min, CE immediate & IT 5 min, CE w/ DACT 5 min \\
\hline 10 & IT 1 min, CE immediate & IT 5 min, CE w/ Simazine 5 min \\
\hline 11 & IT immediate, CE immediate & IS 18 hrs \\
\hline 12 & $\begin{array}{l}\text { IT immediate, CE w/ } 100 \mu M \\
\text { atrazine }\end{array}$ & - \\
\hline
\end{tabular}

In vitro selection process for obtaining atrazine-specific MRE. Immobilization target (IT) is desethyl atrazine bound to magnet beads. Immobilization substrate (IS) is streptavidin-coated magnetic beads plus blocked biotin reagent. Competitive elution (CE) is removal of bound ssDNA from target-coated magnetic beads by free pesticide in solution. Times listed are incubation times in hours (hrs) or minutes (min).

with atrazine as the elutant served as normalization controls. Each elutant was incubated with the immobilized target for five minutes, and the solution was removed from the beads. This solution was placed in a 96-well plate and measured in a fluorescence plate reader. Data were normalized to an internal fluorescent standard and then the no R12.23 control was subtracted. Each set of specific binding studies were performed in triplicate. Data were averaged and 
standard deviations calculated. For each elutant, a t-test was performed to determine statistical differences in the means. To determine binding to other molecules not used in the selection, the same protocol was used. Following incubation of $5 \mathrm{nM}$ fluorescent R12.23 with immobilized target, each incubation was eluted with $100 \mathrm{uM}$ in MSB of the following: atrazine, propazine, terbuthylazine, sebuthylazine, and bovine serum albumin (BSA), which was used as a model of binding to large, globular proteins.

\subsection{Results and Discussion}

\subsubsection{Selection of Atrazine-Specific MREs}

Twelve rounds of Decoy-SELEX were completed to obtain ssDNA MREs which specifically bind to atrazine (Table 2.1). The selection scheme ensured that ssDNA MREs will bind to atrazine in solution and neither the immobilization substrate nor malathion, propanil, 2,4D acid, DACT, or simazine. PCR cycling numbers and ssDNA recovered after preparation and used in the ensuing selection round varied with each round of selection. After every third round of selection (rounds 3, 6, 9, 12 plus after round 10), 30-50 random sequences of the ssDNA molecules in the library were obtained for consensus sequence family analysis. Additionally, random sampling of ssDNA sequences binding to both DACT and simazine was performed.

Consensus sequence families were analyzed and tracked throughout the Decoy-SELEX process. From the round 12 library, three sequences were chosen based on their inclusion in consensus sequence families and predicted tertiary structures. Those sequences, R12.23, R12.28, and R12.57 were assayed for their binding affinity to atrazine (Figure 2.3). Those sequences had Gibb's free energy values of $-11.32 \mathrm{kcal} / \mathrm{mol},-10.71 \mathrm{kcal} / \mathrm{mol}$, and $-10.61 \mathrm{kcal} / \mathrm{mol}$, respectively. 
A R12.23 TGTACCGTCT GAGCGATTCGTACGAACGGCTTTGTACTGTTTGCACTGGCGGATTTAGCCAGTCAGTGT TAAGGAGTGC

R12.23 TGTACCGTCTGAGCGATTCGTACGAACGGCTTTGTACTGT-----TTGCACTGGCGGATTTAGCCAGTCAGT GTTAAGGAGTGC

R12.24 TGTACCGTCT GAGCGATTCGTACAAGAGACT CGGCTTTT GTAATC-----TTGCGGTTT TGGAGCCATTCATTGT TAAGGATTGC

R12.34 TGTACCGTCT GAGCGATTCGTACCGGTTCTT TAGCGGCTGAATAGTATTTTTCTTGCAMCCAGTCAGT GTTAAGGAGTGC

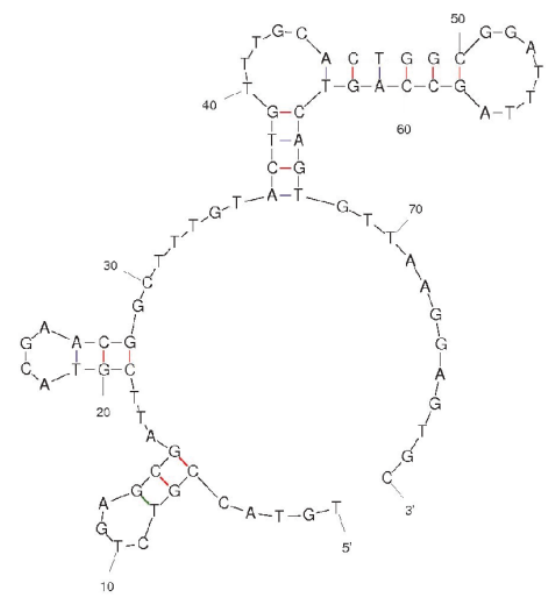

B R12.28 TGTACCGTCTGAGCGATTCGTACCATTAGTGGGT GCTCCTTACCT GATGGTCATCTAGCCAGTCAGTGTTAAGGAGTGC

R12.26 TGTACCGTCTGAGCGATTCGTACGGCTAGAGTTGTTATGTTTCGATGGTCATCTGCAAGCCAGTCAGT GTTAAGGAGTGC

R12.28 TGTACCGTCTGAGCGATTCGTACCATTAGTGGGTGCTCCTTACCT GATGGTCATCT---AGCCAGTCAGTGTTAAGGAGTGC

R12.51 TGTACCGTCTGAGCGATT CGTACTGCAGGCCGTGTGGCGCGGATTACTCGATGGT-ATAGCCAGTCAGTGTTAAGGAGTGC

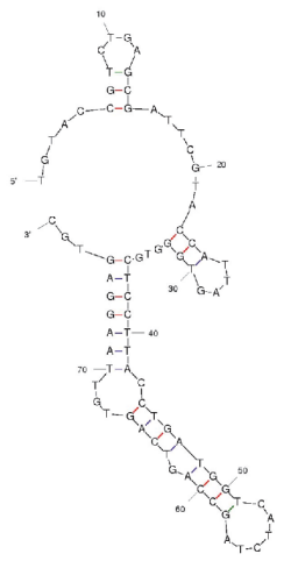

C 12.57 TGTACCGTCTGAGCGATTCGTACGGATTGGGTTTGCACTTTACCTGCGGTGCATCGCAGCCAGTCAGTGTTAAGGAGTGC

R12.28 TGTACCGTCT GAGCGATTCGTACCATTAGTGGGT--GCTCCTTACCTGATGGT-CATCT-AGCCAGTCAGTGTTAAGGAGTGC R12.57 TGTACCGTCTGAGCGATTCGTAC-GGATTGGGTTTGCACTTTACCTGC-GGTGCATCGCAGCCAGTCAGTGTTAAGGAGGC

R12.59 TGTACCGTCT GAGCGATT CGTAC-GGATT GGGTTTGCACTTTACCTGC-GGTGCATCGCAGCCAGT CAGT GTTAAGGAGTG

R12.24 TGTACCGTCT GAGCGATTCGTACAAGAGACTCGGCTTTT--GTAATCTTGC-GGTTTTGGAGCCATTCATTGTT AAGGAT TGC

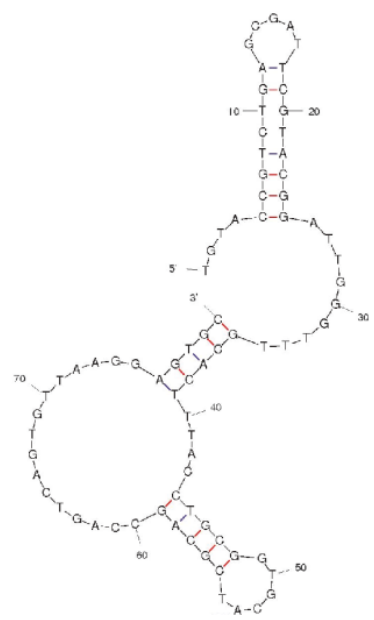


Figure 2.3. Sequences and structures of atrazine MREs. The ssDNA sequence of selected atrazine MREs, which is above a representative consensus sequence family, with the secondary structure as predicted by Mfold below (SantaLucia 1998; Peyret 2000; Zuker 2003). The sequence and structure of (a) R12.23, (b) R12.28, and (c) R12.57.

\subsubsection{Affinity and Specificity of Atrazine-Specific MRE}

Fluorescent saturation binding assays were performed to determine the affinity of R12.23, R12.28, and R12.57 for atrazine. Concentrations of MRE in the high pM to low nM range were assayed, plotted, and fit by nonlinear regression analysis (Figure 2.4). The equilibrium dissociation constant $\left(\mathrm{K}_{\mathrm{d}}\right)$ of R12.23 was determined to be between $0.14 \mathrm{nM}$ and $0.97 \mathrm{nM}$ (Table 2.2). This is in the lower range of MREs targeting small molecules and comparable to MREs targeting proteins (Hamula et al. 2006; Stoltenburg et al. 2007). Such high affinity for atrazine is likely due to the multiple rounds of selection with very stringent binding conditions utilizing the Decoy-SELEX process. Fluorescent binding assays were also performed for R12.28, which showed a $\mathrm{K}_{\mathrm{d}}$ between $1.28 \mathrm{nM}$ and $9.20 \mathrm{nM}$, and R12.57, which showed a $\mathrm{K}_{\mathrm{d}}$ between $4.42 \mathrm{nM}$ and $51.74 \mathrm{nM}$. Therefore, because of its high affinity and reproducible dissociation constant range, R12.23 was chosen for further binding analysis.

Further sequence analysis on R12.23 determined possible binding sequences. This sequence had the greatest stability in the round 12 library as predicted by Mfold. It was present six times in four out of eleven total large round 12 sequence families and was not evident in any previous round of selection. Interestingly however, one five-base consensus sequence in R12.23 was also found in a very large round 10 consensus sequence family (GCTTT). Another five-base consensus sequence in R12.23 was found in large families in both round 6 and 9 (TGTTT). This particular five-base sequence was also found as part of a consensus sequence family for ssDNA that bound to both DACT and simazine. A second five-base consensus sequence in R12.23 also had these characteristics (GCACT). These two sequences appeared consecutively in R12.23, and 
were part of a large dual-hairpin in the predicted structure (Figure 2.3a). This therefore suggests that while this full MRE sequence did not appear in any prior rounds' random sequencing efforts, components therein were important in binding to atrazine and surviving the Decoy-SELEX process while others were important in conferring specificity.

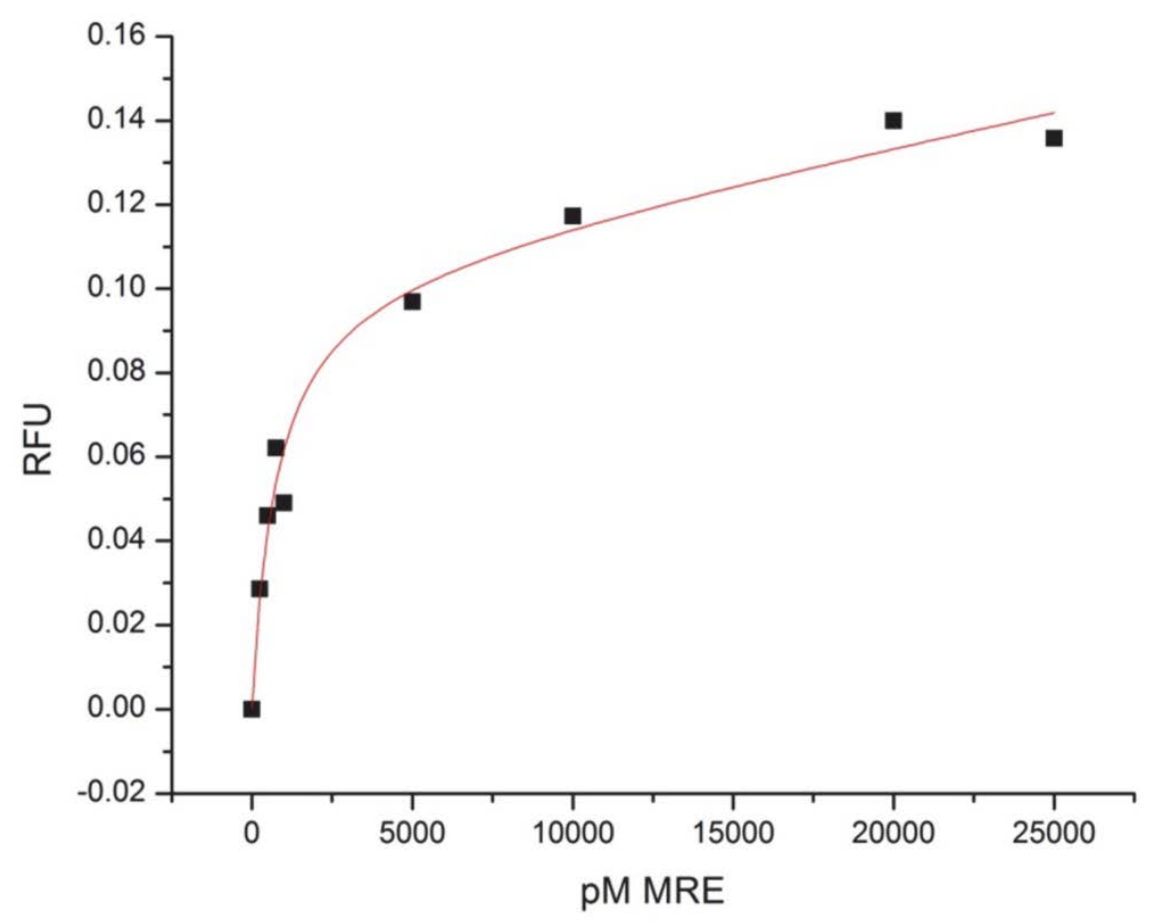

Figure 2.4. Fluorescent equilibrium binding assay of R12.23. Representative saturation binding assay with nonlinear regression best fit. $\mathrm{K}_{\mathrm{d}} \mathrm{R} 12.23$ for atrazine in this data is $0.75 \mathrm{nM}$ $+/-0.22 \mathrm{nM}$.

Table 2.2. Equilibrium dissociation constant $\left(\mathrm{K}_{\mathrm{d}}\right)$ of atrazine MREs.

\begin{tabular}{|l|l|l|l|l|} 
Plot & R12.23 & R12.28 & R12.57 & R12.23.TRNC1 \\
\hline $\mathbf{1}$ & $0.75+/-0.22 \mathrm{nM}$ & $1.28+/-0.85 \mathrm{nM}$ & $51.74+/-7.75 \mathrm{nM}$ & $0.079+/-0.065 \mathrm{nM}$ \\
\hline $\mathbf{2}$ & $0.14+/-0.04 \mathrm{nM}$ & $9.20+/-3.02 \mathrm{nM}$ & $6.18+/-8.67 \mathrm{nM}$ & $0.700+/-0.777 \mathrm{nM}$ \\
\hline $\mathbf{3}$ & $0.97+/-0.61 \mathrm{nM}$ & $4.81+/-1.75 \mathrm{nM}$ & $4.42+/-3.67 \mathrm{nM}$ & $0.848+/-0.826 \mathrm{nM}$ \\
\hline
\end{tabular}

*All data are presented as $\mathrm{K}_{\mathrm{d}}+/$ - Standard Error as given by the binding equation. 


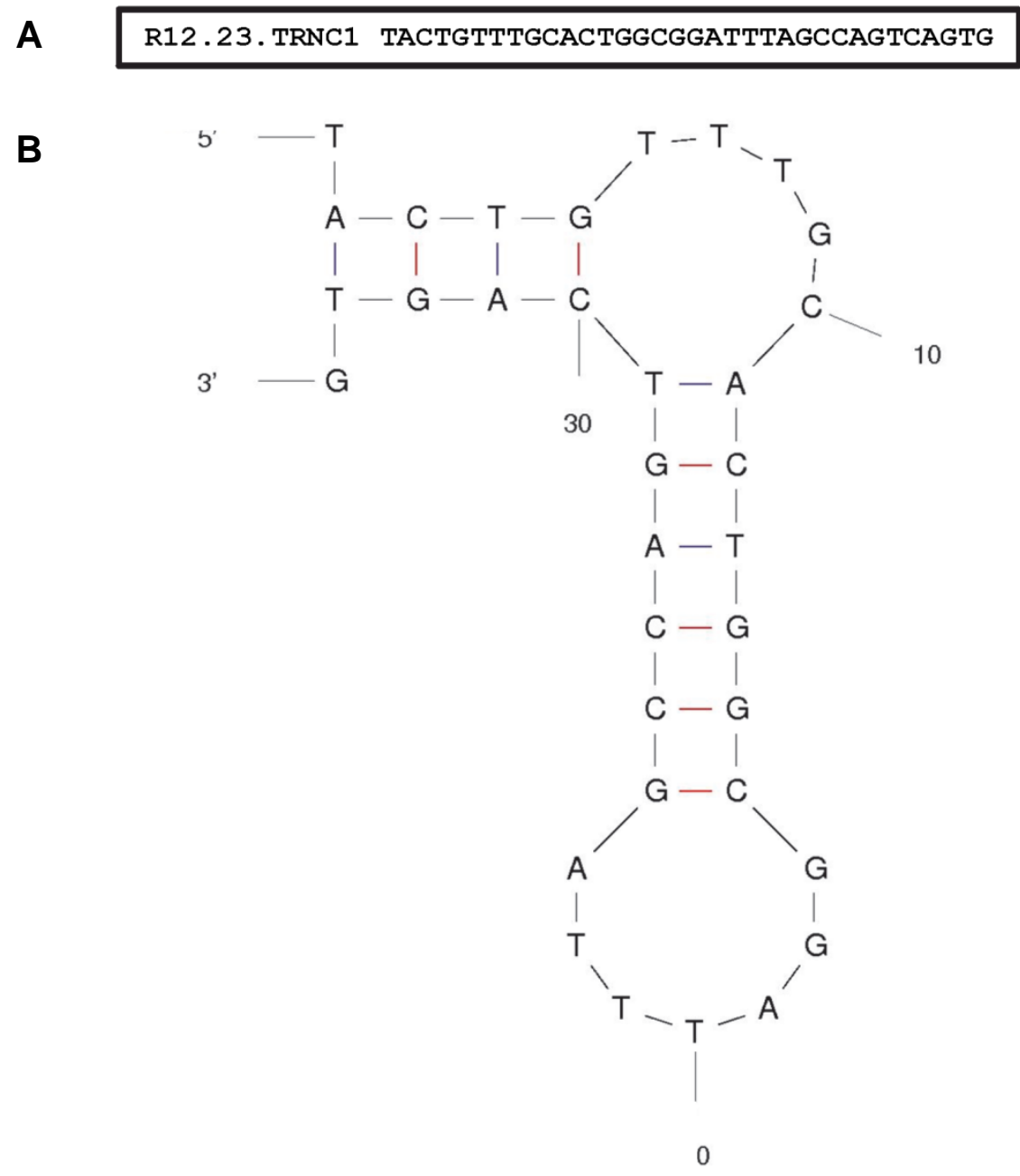

Figure 2.5. Sequence and structure of R12.23.TRNC1. (a) Designed sequence of R12.23.TRNC1. (b) Secondary structure of R12.23.TRNC1 as predicted by Mfold.

To further determine the binding site of R12.23 and in an effort to increase binding affinity, a truncated sequence was designed and termed R12.23.TRNC1 (Figure 2.5). It is comprised of a 34 base sequence which composes the major dual hairpin-loop structural component of the full-length R12.23 discussed in the previous paragraph (Figure 2.5) (synthesized by Katherine Hickey and Peter Gannett of WVU Basic Pharmaceutical Sciences). Fluorescent saturation binding assays using a fluorescein-labeled MRE were performed with this sequence as described above. These assays showed R12.23.TRNC1 had a $\mathrm{K}_{\mathrm{d}} 0.079$ and 0.848 
nM (Table 2.2) (data generated by Eamonn Maher and Ginger He of the Sooter Lab). The affinity of the truncated sequence is overall as good as or better than the full-length sequence. This is similar to previous work that has assayed binding affinity of truncated MRE sequences (Kaur and Yung 2012). Therefore, it is likely that this is the binding domain of R12.23.
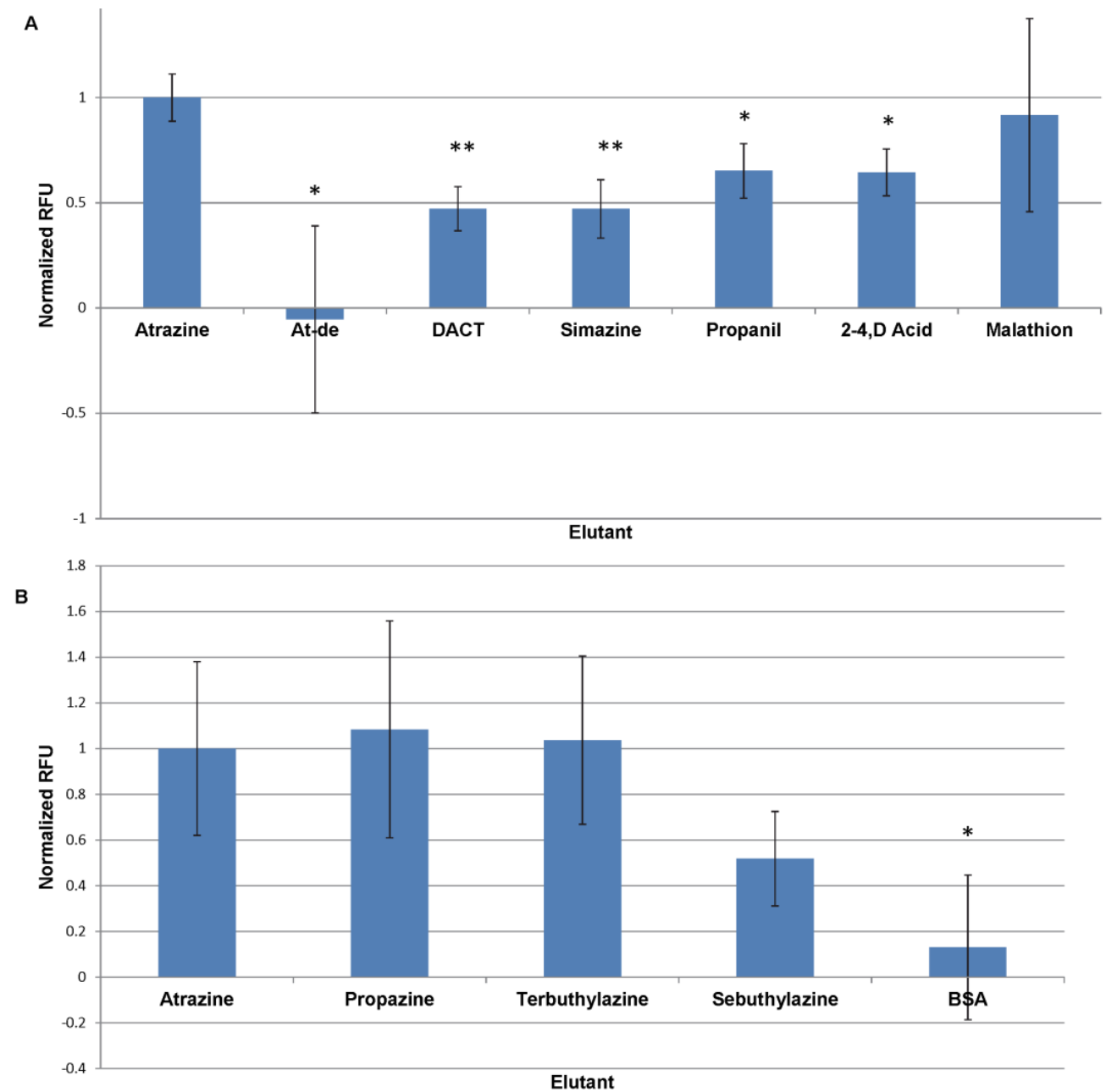

Figure 2.6. Cross-binding studies of R12.23 atrazine MRE to other molecules. Data were normalized to atrazine binding which was set to $100 \%$, with error bars representing standard deviations. Statistical significance levels of $\mathrm{p}<0.05$ are designated by '*', while levels of $\mathrm{p}<0.01$ are designated by '**' (a) Atrazine is the target of selection, desethyl atrazine is the derivitave used for immobilization to magnetic beads. DACT and simazine are metabolites of atrazine. Propanil, 2-4, D acid, and malathion are pesticides found in close environmental proximity to atrazine. (b) Propazine, terbuthylazine, and sebuthylazine have closely-related chemical structures, but were not used in the selection scheme. Bovine serum albumin (BSA) readily binds many molecules and was used as a model of the MRE binding to proteins. 
Fluorescent specificity binding assays were also performed on the atrazine MRE using elution from immobilized target beads. Analysis showed significantly higher binding of R12.23 to atrazine in solution than to four of the five negative targets used in the selection (Figure 2.6a). These included DACT, simazine, propanil, and 2-4,D Acid (Figure 2.2). Binding to malathion in solution did occur but was highly variable. This may be because malathion is known to be reactive toward DNA by cleaving the strand (Pluth et al. 1996; Reus et al. 2008; Moore et al. 2010). This reactivity of malathion may not affect signal of a field- deployable sensor if it is designed to function based on MRE folding and not cleavage. It is important to note that R12.23 is selective for atrazine over simazine, as current molecular recognition elements for atrazine are not (Wortberg et al. 1996). Also, the MRE has no affinity for desethyl atrazine in solution. This is noteworthy because this atrazine derivative was immobilized on magnetic beads and used for the initial positive rounds of selection. Additionally, even though binding to atrazine is approximately two-fold higher than it is to DACT or simazine, the dissociation constant may be much higher than twice that of R12.23 for atrazine. Therefore, incorporation into a sensor may lead to much greater specificity than it appears here. It is clear that stringent competitive elutions selected for an MRE that has high specificity for atrazine in solution compared to the negative targets.

Interestingly, structurally similar molecules (Figure 2.2) which were not used as negative targets bound to the selected MRE (Figure 2.6b). This may be due to the structural complexity of the MRE. Binding is statistically as strong to propazine, terbuthylazine, and sebuthylazine. This suggests that R12.23 requires the chlorotriazine base, as do antibodies that bind to atrazine (Herranz et al. 2008). It also suggests that it requires a certain level of complexity on the aminelinked side-groups of the chlorotriazine base. Based on the structures, it seems binding requires 
at least four total carbons in the side-groups. As simazine also meets this requirement, it seems a second requirement is having at least one side-group with three carbons. This is evidenced by the lack of binding to simazine, DACT, and desethyl atrazine, which do not meet these complexity requirements. Propazine, with two amine-linked isopropyl groups, terbuthylazine, with an amine-linked ethyl and an amine-linked tert-butyl, and sebuthylazine, with an aminelinked sec-butyl, exceed these complexity requirements. It is unclear how more complex chlorotriazines would be bound by the MRE. Exceedingly-complex chlorotriazines, however, likely would be too large to fit in the MRE binding pocket. Bovine serum albumin (BSA) was used as an example of potential MRE binding to proteins. The MRE did not show significant binding to BSA. It is likely that, in spite of some cross-reactivity to more complex chlorotriazines, R12.23 will be useful in a sensor for a class of molecules. After rapid, fieldbased detection with the MRE, more rigorous analytical approaches such as gas chromatography can be used to identify all present contaminants.

The high affinity and specificity of the atrazine MRE are both important characteristics for its further use in detection applications, such as contaminated ecosystems or individuals. Nucleic acid MREs have previously been developed and used in environmental biosensing for small molecules (Strehlitz et al. 2012; Yildirim et al. 2012). They have also been used for detection of analytes in serum (Soh et al. 2009). In both contexts, it is necessary for the MRE to differentiate between the target and closely-related molecules or other contaminants. The novel selection method reported herein is capable of generating such MREs. Once atrazine-polluted environments or contaminated at-risk individuals are identified, they can be actively remediated or treated with chemical, physical or biological methods (Mandelbaum et al. 1995; Saltmiras and Lemley 2002; Herzberg et al. 2004). 


\subsection{Conclusions}

A novel variation of the SELEX process, called Decoy-SELEX, is presented to select a molecular recognition element with high affinity and specificity for the herbicide atrazine. The MRE binds with a sub-nanomolar equilibrium dissociation constant and is selective for atrazine over several metabolites and other pesticides. The results validate the Decoy-SELEX process while identifying a new mechanism for atrazine detection in environmental and biological mediums. This work will improve upon current analysis of atrazine contamination and allow for informed remediation and treatment efforts. 


\section{CHAPTER 3}

\section{Introduction to the Properties and Applications of Single-Wall}

Carbon Nanotubes 


\subsection{Introduction}

Carbon nanotubes are cylindrical fullerenes that can be visualized as rolled graphene sheets with walls of single atom thickness. Their discovery stemmed from the discovery of buckminsterfullerene in 1985 (Kroto et al. 1985). Sumio Iijima originally described multi-wall carbon nanotubes (MWCNT) in 1991 (Iijima 1991; Ajayan 1999). Single-wall carbon nanotubes (SWCNT) were independently described by Iijima and Ichihashi as well as an IBM team in 1993 (Bethune et al. 1993; Iijima and Ichihashi 1993). Since this time, research into their production, properties, and applications has increased.

In those original investigations by Iijima the carbon nanotubes were produced by the arcdischarge method. MWCNT were discovered as a deposit on the graphite cathode tip of this chamber (Iijima 1991; Ando and Zhao 2006). In contrast, SWCNT were discovered as a soot from the gas phase of an arc-discharge chamber using either an iron or cobalt catalyst (Bethune et al. 1993; Iijima and Ichihashi 1993; Ando and Zhao 2006). Characterization and confirmation of the products was performed using transmission electron microscopy and electron diffraction. There have since been new production and characterization methods but these remain useful in the field.

Physical differences between MWCNT and SWCNT confer unique properties. The diameter of an individual SWCNT is typically in a narrow range between 0.5 and $2 \mathrm{~nm}$, while that of a MWCNT is 2-25 nm (Ajayan et al. 1999). This is because SWCNT have walls only one atom thick, while MWCNT have concentric walls approximately $0.34 \mathrm{~nm}$ apart (Ajayan 1999). Both, however, have a high aspect ratio, with individual tubes typically having lengths in the micron range. Due to variations in how the edges of graphene sheet meet, walls of both carbon nanotube forms may have helicity, which plays a large role in their physical properties. The 
walls of MWCNT may have different helicity, and therefore less uniform with less ideal optical and electronic properties for individual tubes (Ajayan 1999). Individual SWCNT species, however, have uniform and characteristic properties which give them greater potential for application. The helicity of a SWCNT species is also referred to as its chirality, or its $(\mathrm{n}, \mathrm{m})$ species (Figure 3.1). This designation derives from the angle at which the tube is rolled. Achiral carbon nanotubes are either in the armchair $(n, n)$ or zigzag $(n, 0)$ conformations. Some properties have been investigated, however there is much yet to be determined.

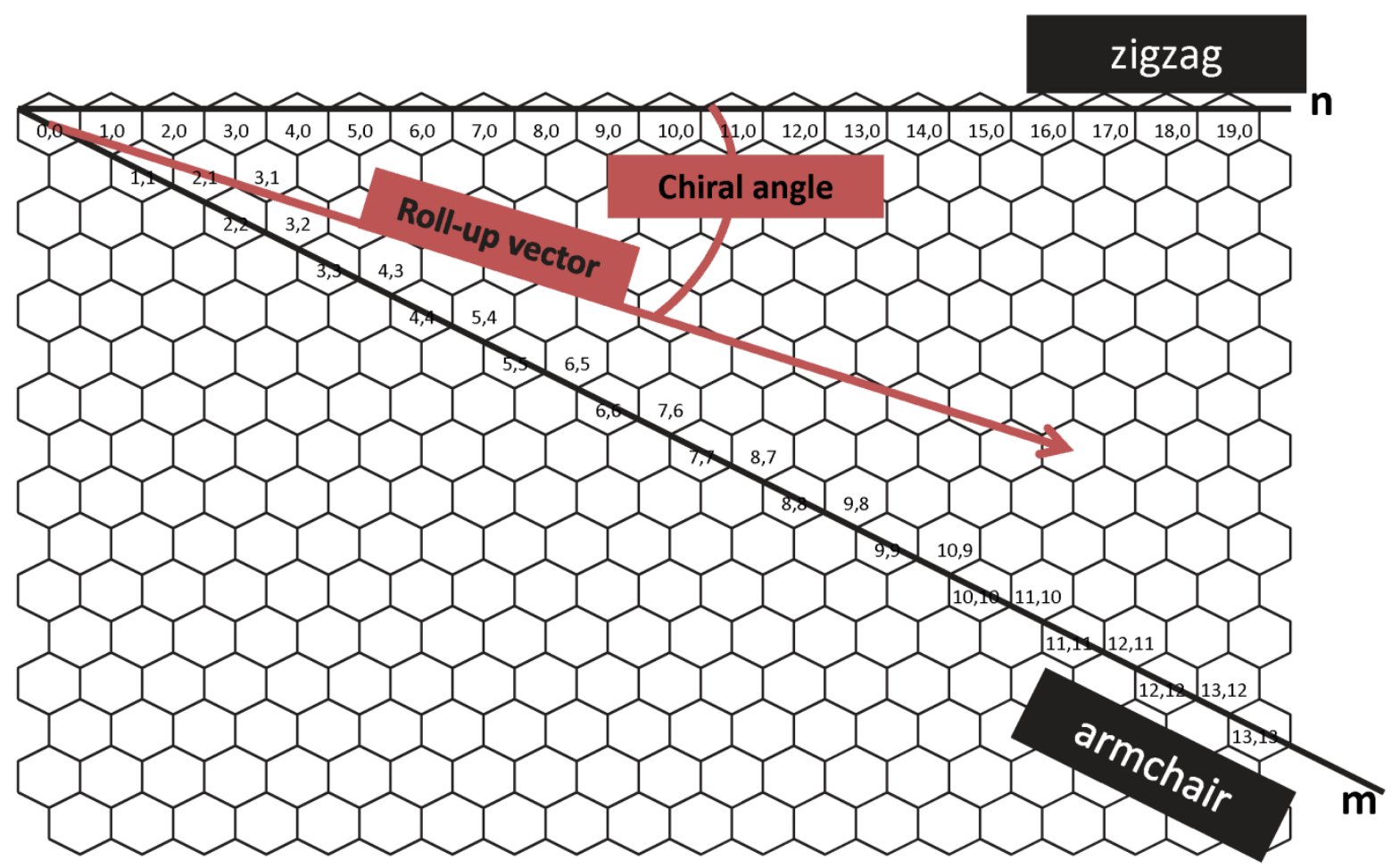

Figure 3.1. Derivation of SWCNT $(\mathbf{n}, \mathbf{m})$ chiral species. A visual representation of a graphene sheet depicts the derivation of SWCNT chirality. Carbon bonds form hexagons, and SWCNT can be visualized as rolled-up graphene sheets. The angle at which the sheet is rolled determines the chirality, or the $(n, m)$ designation of the tube. Some n,m species are shown here, with the lines from which zigzag and armchair chiral species are derived. Image is derived from previously-published depiction (Weisman 2010).

The potential application of SWCNT has led to an increase in their production and use. There has been a push to determine their interaction with biological materials. This is because 
they have enormous potential in biological sensors and medical treatments. It is also true, however, because there will be an increase in environmental contamination by these particles. It is therefore necessary to understand the production of SWCNT, their special properties, and the applications of SWCNT.

\subsection{SWCNT Production}

The initial synthesis of SWCNT by the arc-discharge method with a catalyst is still one of the three major forms of their industrial synthesis. Synthesis by laser-ablation and chemical vapor deposition for catalytic growth has since been adopted.

\subsubsection{Arc-Discharge Method}

The arc-discharge method of SWCNT product was the first developed and remains to be in use because it is simple and has a relatively high yield (Baddour and Briens 2005). To produce SWCNT, a chamber is filled with an inert gas and contains two graphite electrodes (Bethune et al. 1993; Iijima and Ichihashi 1993). A metallic catalyst such as iron or cobalt is placed on either of the electrodes. Then a direct current is applied between the electrodes and SWCNT are deposited from the vapor phase of the chamber. The resulting SWCNT products are relatively short, bundled, and need purification due to impurities (Saifuddin et al. 2013). This method was scaled-up from initial production in 1997 by the work of Journet et al. (Journet et al. 1997), however larger-scale production remains difficult.

\subsubsection{Laser-Ablation Method}

Laser-ablation synthesis of SWCNT is the most expensive method of SWCNT production, however the product is of relatively high purity (Baddour and Briens 2005). A target of graphite with metal catalysts is inserted into a furnace with an inert gas in this method (Guo et al. 1995; Thess et al. 1996). High-intensity laser pulses are then directed at the graphite and 
SWCNT are deposited on a water-cooled collector (Popov 2004). This method is only useful for production of SWCNT and not MWCNT. Additionally, it can be more finely tuned by laser and gas properties to produce SWCNT with specific characteristics (Saifuddin et al. 2013).

However, the product is tangled and needs purification. This coupled with the high cost makes this method is not as extensively used as the other two.

\subsubsection{Chemical Vapor Deposition Method}

Synthesis of SWCNT by chemical vapor deposition (CVD) is the most inexpensive and easiest method to scale up for industrial purposes. This method involves the production of SWCNT by flowing hydrocarbons and an inert gas over a metal catalyst in a furnace (Popov 2004). It was initially used for production of carbon filaments before the discovery of SWCNT (Baker 1989). Since then, however, it has been adapted and scaled up for industrial carbon nanotube production (Joseyacaman et al. 1993; Amelinckx et al. 1994; Ivanov et al. 1994; Li et al. 1996). This process generally favors the production of MWCNTs, however the characteristics of SWCNT are highly controllable under specific conditions (Kong et al. 1998; Baddour and Briens 2005; Saifuddin et al. 2013). Overall, the CVD method typically has high purity and produces longer nanotubes. The only drawback to this method is that the SWCNT typically have many defects (Saifuddin et al. 2013). Despite this, the simplicity, costeffectiveness, and scalability of the CVD method make it the most widely-used for industrial purposes.

\subsubsection{Purification and Enrichment}

After synthesis of SWCNT, they may undergo multiple processes before their study and application. These include purification of the nanotubes from synthesis contaminants and 
enrichment of particular SWCNT types. Both of these steps may affect the SWCNT properties and neither of them is completely optimized.

Purification of SWCNT is necessary after the synthetic process due to carbonaceous and catalytic metal impurities. The most commonly-used methods for this are oxidation, filtration and chromatography, chemical functionalization, and microwave heating (Haddon et al. 2004; Park et al. 2006). There are, however, other techniques that may be used in conjunction with these, such as centrifugation, ultrasonication, and magnetic separation (Saifuddin et al. 2013).

Another possible form of post-synthetic processing is enriching SWCNT of a particular diameter, length, or chirality It is important to be able to do this because these characteristics are strong determinants of SWCNT physical properties (Zhang and Zheng 2010). This may be partially done through controlled synthesis with specific temperatures, catalysts, or substrates (Bandow et al. 1998; Kukovitsky et al. 2002). However, it is also possible to enrich particular SWCNT types by oxidation and etching (Zhang et al. 2006; Miyata et al. 2007). Enrichment of SWCNT by chirality and diameter is possible through ultracentrifugation (Arnold et al. 2006). Finally, the affinity of particular surfactants for individual $(n, m)$ species has been used to separate SWCNT (Ju et al. 2008; Marquis et al. 2008). Of these, DNA is the most promising, as individual sequences have greater affinity for particular diameters and chiralities (Huang et al. 2005; Albertorio et al. 2009; Tu et al. 2009; Tu et al. 2011). Obtaining pure SWCNT chiral species is important for many applications, however the process remains difficult to obtain highly pure samples (Komatsu and Wang 2010; Zhang and Zheng 2010).

\subsection{Properties of SWCNT}

Single-wall carbon nanotubes have many properties that make them ideal for a range of applications (Table 3.1). These include special mechanical, electrical, and optical characteristics. 
As mentioned previously, the properties depend strongly on the SWCNT diameter, chirality, and length.

Table 3.1. Special properties of single-wall carbon nanotubes (SWCNT) and their correlation to applications.

\begin{tabular}{|l|l|l|}
\hline Property & Description & Application \\
\hline Mechanical & $\begin{array}{l}\text {-Very strong } \\
\text {-High stiffness } \\
\text {-Flexible } \\
\text {-Large surface area } \\
\text {-Low density }\end{array}$ & $\begin{array}{l}\text {-Replacement of carbon } \\
\text { fibers for material } \\
\text { reinforcement } \\
\text {-Polymer integration for } \\
\text { flexibility and strength }\end{array}$ \\
\hline Electrical & $\begin{array}{l}\text {-Individual chiralities are } \\
\text { either metallic or } \\
\text { semiconducting }\end{array}$ & $\begin{array}{l}\text {-Conductors in electronics } \\
\text {-Signal transducers in } \\
\text { sensors }\end{array}$ \\
\hline Optical & $\begin{array}{l}\text {-Fluorescence in the near- } \\
\text { infrared region } \\
\text {-Exact fluorescence } \\
\text { properties dependent on } \\
\text { chirality and length }\end{array}$ & $\begin{array}{l}\text {-Optical fluorescence } \\
\text { sensors } \\
\text {-In vivo imaging } \\
\text {-Chirality, length, and } \\
\text { dispersion characterization }\end{array}$ \\
\hline Functionalization & $\begin{array}{l}\text {-Ability to be chemically } \\
\text { modified } \\
\text {-Ability to be wrapped or } \\
\text { adsorbed to by biological } \\
\text { molecules }\end{array}$ & $\begin{array}{l}\text {-Potential for aiding in all } \\
\text { previous applications } \\
\text {-Biological sensors }\end{array}$ \\
\hline
\end{tabular}

\subsubsection{Mechanical Properties}

The mechanical properties of SWCNT have made them of high interest for many industrial applications. They are considered very strong with high stiffness due to characteristic sp $^{2}$ carbon bonding (Robertson et al. 1992; Popov 2004). Individual SWCNT have an average Young's modulus value of $1.8 \mathrm{TPa}$, which is an extraordinarily large measure of stiffness for a material (Jacobsen et al. 1995). Important for engineering and industrial purposes, the value for bundled SWCNT is estimated to be $600 \mathrm{GPa}$; lower due to weaker associations and formations of individual tubes (Salvetat et al. 1999). This, however, can theoretically be up to 38 TPa for identical achiral SWCNT, which could be extremely useful in engineering applications (Popov et 
al. 2000). They are unique in that they also have a high flexibility in spite of their strength and stiffness (Coleman et al. 2006). Additionally, SWCNT have a very high surface area and a relatively low density of $0.6 \mathrm{~g} / \mathrm{cm}^{3}$ (Ajayan 1999).

\subsubsection{Electrical Properties}

Single-wall carbon nanotubes may be metallic or semiconducting based on their chirality. Of all possible SWCNT species, 33\% are metallic and 67\% are semiconducting (Saito et al. 1992; Zhang and Zheng 2010), with all armchair conformations being metallic and zigzag and chiral being either (Ajayan 1999). These properties make it possible to use individual species or broad classes of SWCNT in electronics or sensors. Additionally, it is possible to use nanotubes to create intramolecular electronic junctions (Ouyang et al. 2002). Overall, the electronic properties of SWCNT have been studied largely by scanning tunneling microscopy (STM) (Ouyang et al. 2002). Due to these properties, SWCNT have a great deal of potential in electronic and sensing applications.

\subsubsection{Optical Properties}

The specialized optical properties of SWCNT make them particularly important for sensing and biomedical applications. Semiconducting nanotubes have optical absorption peaks corresponding to excitation of electrons from valence to conduction bands, a separation referred to as the band gap (Weisman 2010). These well-defined peaks are referred to as $E_{11}, E_{22}$, and so on, which fall in the near-infrared (IR) and visible light regions, respectively. Fluorescence has been observed across the near-IR band gap of SWCNT (O'Connell et al. 2002). As this is highly dependent on the diameter and chirality of the SWCNT, it is possible to characterize the nanotubes present in a sample based on its optical properties (Bachilo et al. 2002). Furthermore, the near-IR region is relatively unabsorbed by tissue, thus making SWCNT of great potential for 
biological imaging and therapeutics (Welsher et al. 2011). SWCNT can generate and detect light while functioning as a transistor, and therefore have great potential for combined electronic and optical devices (Avouris et al. 2008). It is also possible to use absorbance versus fluorescence as a relative measurement of SWCNT dispersion in solution. This is because bundled SWCNT absorb light, but their fluorescence output is dampened (Weisman 2010). It is therefore obvious that optical characterization and applications of SWCNT have great potential.

\subsection{SWCNT Applications}

Stemming from these properties of SWCNT is the ability to use them in a variety of applications (Table 3.1). They have been integrated into industrial, electronic, sensing, and biomedical processes, among others. It is also important to note that for many applications, it is necessary to have pure or enriched SWCNT species in order to have uniform properties.

\subsubsection{Industrial Applications}

Due to their unique mechanical properties, SWCNT have been incorporated into polymers to form composites with desirable properties (Breuer and Sundararaj 2004). They are often used instead of carbon fibers, which are used to reinforce and enhance materials for aerospace, automotive, or sporting and protective equipment. They can also be integrated into composites for their ability to dissipate electrostatic interactions, which can be important in safer materials. Considerable research has gone into optimizing the interface of SWCNT and polymers to extract ideal properties (Thostenson et al. 2001). This work has significantly increased the stiffness of composite materials and led to an increase of SWCNT-related products in the marketplace (Coleman et al. 2006). 


\subsubsection{Electronic Applications}

The application of SWCNT in electronics has also become more widespread. This is because of their excellent conductivity, surface area, and ability to take different macroscopic shapes (Schnorr and Swager 2011). SWCNT have thus been studied for use in lithium ion batteries and transparent electrodes, which are components of organic solar cells and organic light-emitting diodes (OLEDs) (Ng et al. 2005; Kim et al. 2009; Landi et al. 2009; Chien et al. 2010). Additionally, they can be used as capacitors and even to build smaller and more efficient electronic circuits, among many other uses (Franklin et al. 2001; Baughman et al. 2002; Du and Pan 2006; Schnorr and Swager 2011).

\subsubsection{SWCNT in Sensors}

The use of SWCNT in sensors can be closely related to their electronic applications. However, SWCNT-based sensors may also rely on their optical properties. An example of an electronic-based SWCNT sensor is a field effect transistor (FET) (Tans et al. 1998). The nanotubes can adsorb, be wrapped by, or covalently bound to sensing molecules, which changes the conductance of the nanotube upon binding (Pengfei et al. 2003; So et al. 2008; Schnorr and Swager 2011). They have also been used for detection of gases and chemical vapors through chemically reactive coatings (Novak et al. 2003; Snow et al. 2005). As mentioned above, polymers or other biological sensing molecules can be attached to SWCNT. This includes adsorption or the covalent attachment of antibodies as well as covalent attachment or wrapping of DNA around nanotubes (Zhang et al. 2009). These can function in sensors as described above, or can be part of optical SWCNT sensors (Huang et al. 2011). An important example of this is the near-infrared detection of analytes with DNA and DNA molecular recognition 
element(MRE)-wrapped SWCNT (Jeng et al. 2006; Chen et al. 2009). These are among many other examples of research being done to develop SWCNT-based sensors.

\subsubsection{Biomedical Applications}

One area of great potential for SWCNT use is in biomedical applications, which draws on multiple properties of this material. An important necessity of this use is that the nanotubes are not toxic to humans. Multiple studies have shown that SWCNT functionalized or non-covalently altered with biological molecules reduce their toxic effects (Vardharajula et al. 2012). Therefore, they have been developed for use as in vitro and in vivo imaging tools, as their near-infrared fluorescence is detectable through cells and tissue (Welsher et al. 2011; Kang et al. 2012). Additionally, they have been used to deliver small molecule and biologic drugs as well as genes to specific diseases (Pantarotto et al. 2004; Portney and Ozkan 2006; Bhirde et al. 2010). Research is ongoing to ensure the safety of SWCNT in biomedical applications, which will lead to an invaluable tool for clinical diagnostics and therapeutics.

\subsection{Conclusions}

Single-wall carbon nanotubes are an extraordinary and relatively new class of materials into which research is ongoing and increasing constantly. This is due to their unique and desirable properties, which are drawn from their production, purification, and enrichment. They have particular value in both biomolecular sensing and biomedical applications. Both of these uses require further work into understanding the interactions of SWCNT and biological molecules and systems.

The work described here was completed to further determine specific properties of SWCNT. As noted, their interaction with DNA is of particular interest for sorting and separation, sensors, and other applications. The effect of multiple chiralities of DNA wrapped 
with different DNA sequences on the polymerase chain reaction was determined. This will allow for future work in separation of SWCNT and in sensors. Additionally, the effect of SWCNT on a green algae model organism was determined. This will help to determine future environmental impacts of widespread DNA-wrapped SWCNT use and whether these constructs are safe for use. Together, these investigations into the interactions of biological systems and DNA-wrapped SWCNT have elucidated properties of SWCNT important for their future use. 


\section{CHAPTER 4}

Investigation of the Effects of DNA-dispersed Single-Wall Carbon Nanotubes on the Polymerase Chain Reaction 


\subsection{Introduction}

Single-wall carbon nanotubes (SWCNT) are cylindrical fullerenes with walls of single carbon atom thickness (Iijima 1991; Iijima and Ichihashi 1993; Ajayan 1999). They have enormous potential in industrial, sensing, and biomedical applications due to their unique physical, electronic, and optical properties (Heller et al. 2005; Wang 2005; Coleman et al. 2006; Huang et al. 2011). SWCNT may be either metallic or semiconducting, with $33 \%$ and $67 \%$, respectively, of all potential species having those characteristics (Saito et al. 1992; Zhang and Zheng 2010). The unique properties of SWCNT are dependent upon the chirality and diameter of the SWCNT, however purification of individual species remains difficult (Zhang and Zheng 2010). Individual types of semiconducting SWCNT have unique optical fluorescence properties (O'Connell et al. 2002). Therefore, in applying SWCNT, it is possible to take advantage of their electrical or optical properties, separately or combined (Avouris et al. 2008).

Single-stranded DNA has been used to disperse hydrophobic SWCNT in solution (Zheng et al. 2003). The interaction of DNA sequences with SWCNT may have implications in sensing and purification techniques. Individual sequences have been identified that preferentially wrap certain SWCNT chiralities (Huang et al. 2005; Albertorio et al. 2009; Tu et al. 2009; Tu et al. 2011). DNA-associated SWCNT have also been used in molecular sensing (So et al. 2005; Jeng et al. 2006; So et al. 2008). The potential use of a DNA-wrapped SWCNT (DNA:SWCNT) platform shows promise in multiple applications; however research is continuing to optimize use of these complexes.

In sensing applications and analysis of environmental samples, it is useful to employ polymerase chain reaction (PCR) techniques in order to exponentially amplify the SWCNTassociated DNA sequence. Additionally, there has been work to develop PCR-based 
microfluidic sensing devices (Khandurina et al. 2000), as well as DNA-functionalized SWCNT sensing devices (So et al. 2005). Integration of these two sets of technologies would create new potential analyte detection mechanisms. Therefore, it is important to determine the effect of DNA-associated SWCNT on the polymerase chain reaction.

The aim of this study was to investigate amplification of DNA by the polymerase chain reaction (PCR) in the presence of DNA-dispersed SWCNT. Factors investigated include DNA sequence-dependence, SWCNT chirality-dependence, and DNA:SWCNT concentrationdependence; amplification of the wrapping sequence itself; and age of the DNA-SWCNT preparation. This work will provide a basis for further DNA-dispersed SWCNT studies which require PCR amplification.

\subsection{Methods}

\subsubsection{DNA-Dispersed SWCNT Preparation}

DNA:SWCNT preparations were made similarly to previously described methods (Zheng et al. 2003; Lahiji et al. 2007). Briefly, 2 mg CoMoCAT SWCNT were mixed with 2 mg DNA in $2 \mathrm{~mL}$ phosphate-buffered saline (PBS). SWCNT used were: without chiral purification "Mix" or $(6,5)$ chirality-enriched (Sigma-Aldrich; St. Louis, MO). DNA sequences used were: estradiol (E2) Molecular Recognition Element (MRE) (Table 4.1) (Kim et al. 2007), TG15 (Table 4.1) (Eurofins MWG Operon; Huntsville, AL), or salmon testes genomic DNA (Sigma-Aldrich; St. Louis, MO). This mixture was sonicated on ice for two hours with a Virsonic XL2020 ultrasonic liquid processor (Misonix; Farmingdale, NY) equipped with a $3.2 \mathrm{~mm}$ microtip at approximately 20\% power. The resulting solution was centrifuged at 16,000xg for 90 minutes to remove insoluble SWCNT. The solution was then filtered through an Amicon Ultra-4 Centifugal Filter unit with 100,000 molecular weight filter (Millipore; Billerica, MA) three times at 4000 RPM for 15 minutes each to remove DNA that did not associate with the SWCNTs. The recovered DNA:SWCNT solution was brought to a volume of $500 \mu \mathrm{L}$ with PBS. The concentration of the DNA:SWCNT sample was obtained by gravimetric analysis of $10 \mu \mathrm{L}$ of the solution 
weighed on an Ohaus Discovery Microbalance (Ohaus; Parsippany, NJ). Samples were stored in screwtop containers at room temperature before use. To determine the effect of DNA:SWCNT preparation age on PCR, samples were used that were prepared between four and ten months before assayed or less than one month before assayed. In total, 12 DNA:SWCNT samples were prepared.

Table 4.1. Synthesized DNA sequences used in DNA:SWCNT PCRs.

\begin{tabular}{cr}
\hline Sequence Name & DNA Sequence Used \\
\hline Estradiol (E2) & 5'-GCTTCCAGCTTATTGAATTACACGCAGAGGGTAGCGGCTCTGCGCAT \\
MRE & TCAATTGCTGCGCGCTGAAGCGCGGAAGC-3' \\
TG15 & 5'-TGTGTGTGTGTGTGTGTGTGTGTGTGTGTG-3' \\
F.E2 & 5'-GCTTCCAGCTTATTGAATTACACGCAGAGGGTAGC-3' \\
R.E2 & 5'-GCTTCCGCGCTTCAGCGCGC-3' \\
\hline
\end{tabular}

\subsubsection{SWCNT Characterization}

Absorption and near-infrared (NIR) fluorescence measurements were performed as previously described with few modifications (Weisman 2010). Briefly, each DNA:SWCNT preparation was diluted to a $1 \%$ solution with distilled-deionized water (Millipore; Billerica, MA). This solution was added to a 10 mm path length cuvette (Starna Cells; Atascadero, CA) and analyzed on a NanoSpectralyzer 1 (NS1) (Applied NanoFluorescence; Houston, TX). The absorbance spectrum was collected from 400 - $1600 \mathrm{~nm}$. Additionally, fluorescent emission spectra were measured using excitation wavelengths of $638 \mathrm{~nm}, 690$ $\mathrm{nm}$, and $784 \mathrm{~nm}$. The resulting spectra were analyzed with ANFSoft (Applied NanoFluorescence; Houston, TX). Fluorescence efficiency measurements, which are fluorescence intensity (RFU) collected across the emission spectrum for a single wavelength divided by the absorbance spectrum, are reported as a relative measure of SWCNT dispersion in solution.

\subsubsection{Polymerase Chain Reaction in the Presence of DNA-Dispersed SWCNT}

In total, 16 sets of PCR experiments were performed involving 7 individual PCR reactions per set. PCR reactions were composed of: $60 \mathrm{nM}$ E2 MRE, $400 \mathrm{nM}$ forward and reverse E2 MRE primers (F.E2 and R.E2) (Table 4.1), $250 \mu \mathrm{M}$ deoxyribonucleotide triphosphates, 1X GoTaq Reaction Buffer (Promega; Madison, WI), 3.5 units Taq DNA Polymerase, DNA:SWCNT suspension, and Milli-Q water 
to $50 \mu \mathrm{L}$. DNA:SWCNT were added to final concentrations of: $0.01 \mathrm{mg} / \mathrm{mL}, 0.1 \mathrm{mg} / \mathrm{mL}, 1 \mathrm{mg} / \mathrm{mL}, 5$ $\mathrm{mg} / \mathrm{mL}, 10 \mathrm{mg} / \mathrm{mL}$. For each set, a negative control was performed with no E2 MRE or DNA:SWCNT and a positive control was performed with no DNA:SWCNT added. Reactions were performed on an MJ Mini Thermal Cycler (Bio-Rad; Hercules, CA) with conditions: initial denaturation of $95^{\circ} \mathrm{C}$ for 5 minutes; 20 cycles of $95^{\circ} \mathrm{C}$ for 1 minute 30 seconds, $68^{\circ} \mathrm{C}$ for 45 seconds, and $72^{\circ} \mathrm{C}$ for 1 minute 30 seconds; and final extension of $72^{\circ} \mathrm{C}$ for 7 minutes. Amplification of DNA directly associated with SWCNT was performed using E2 MRE:SWCNT preparation with no additional E2 MRE added to the reaction mix.

\subsubsection{PCR Analysis by Agarose Gel Electrophoresis}

Each set of control and DNA:SWCNT PCRs was run on a 4\% agarose gel for 30 minutes at 135 volts in $1 \mathrm{X}$ Tris:Borate:EDTA buffer. Five $\mu \mathrm{L}$ of each PCR and $3 \mu \mathrm{L}$ of 100bp and 25bp ladder (Promega), were mixed with $2 \mu \mathrm{L}$ 6X loading dye (Promega) prior to loading in the well. Gels were pre-stained with $5 \mu \mathrm{g} / \mathrm{mL}$ ethidium bromide and imaged with UV illumination on a BioRad Gel Doc System and analyzed on Quantity One Software (Bio-Rad; Hercules, CA).

\subsection{Results and Discussion}

\subsubsection{Analysis of DNA:SWCNT Preparations}

All DNA:SWCNT samples were analyzed by absorbance and NIR fluorescence spectroscopy to determine their SWCNT chirality composition and dispersion in solution (singly-dispersed SWCNTs) (Figures 4.1 and 4.2). DNA:SWCNT samples greater than four months old were more well-dispersed than the identical DNA:SWCNT preparation of less than one month. This would suggest that dispersion of SWCNT in solution with DNA increases with time. Additionally, for both old and new samples, TG15 and E2 MRE dispersed the $(6,5)$ enriched SWCNT better than mixed chirality SWCNT (Figures 4.1 and 4.2, fluorescence efficiency). These results are consistent with previously published results that have shown short, 
synthetic DNA sequences rich in thymine and guanine have a high affinity for $(6,5)$ chiral SWCNTs (Kim et al. 2008). Conversely, the salmon testes genomic DNA:Mix SWCNT samples were more well-dispersed than Salmon testes genomic DNA:(6,5) SWCNT. These results suggest that although the genomic DNA shows the highest association with $(6,5)$-chiral SWCNTs (Figures 4.1 and 4.2, topography plots) it also forms stable associations with other chiralities.

\subsubsection{Agarose Gel Analysis of Old DNA:SWCNT PCRs}

Agarose gel analysis of PCRs performed with increasing concentrations of DNA:SWCNT preparations between four and ten months old suggest that accurate amplification products of 76 base pairs (bp) can be obtain at concentrations of $0.1 \mathrm{mg} / \mathrm{mL}$ and below for all preparations (Figure 4.1 agarose gels). In most cases, efficiency of PCR amplification is either unaffected or slightly aided at 0.01 and $0.1 \mathrm{mg} / \mathrm{mL}$ compared to the positive control reaction (Figure 4.1 relative intensities). The exception to this is the TG15:(6,5) sample (Figure 4.1b), where a very faint band is produced at $0.1 \mathrm{mg} / \mathrm{mL}$. Additionally, bands are produced at $1 \mathrm{mg} / \mathrm{mL}$ for TG15:Mix and E2:(6,5) PCRs. No distinct bands are produced at 5 or $10 \mathrm{mg} / \mathrm{mL}$ concentration PCRs for any preparation. Prior work has shown that SWCNT sonicated in water just before being added to a PCR reaction increased amplification efficiency of a 410 bp product at concentrations up to $3 \mathrm{mg} / \mathrm{mL}$ (Cui et al. 2004). The same study showed that SWCNT in a PCR reaction interacts with both the DNA template as well as Taq DNA Polymerase. This study, however, has DNA wrapped around SWCNT, so this is unlikely to be the source of inhibition. Another study showed that that SWCNT functionalized with carboxylic groups produce stronger interactions with Taq and more inhibition of PCR than did pristine SWCNT at 0.1-0.8 $\mathrm{mg} / \mathrm{mL}$ (Yi et al. 2007). Therefore, it is possible there is some interaction between 
A

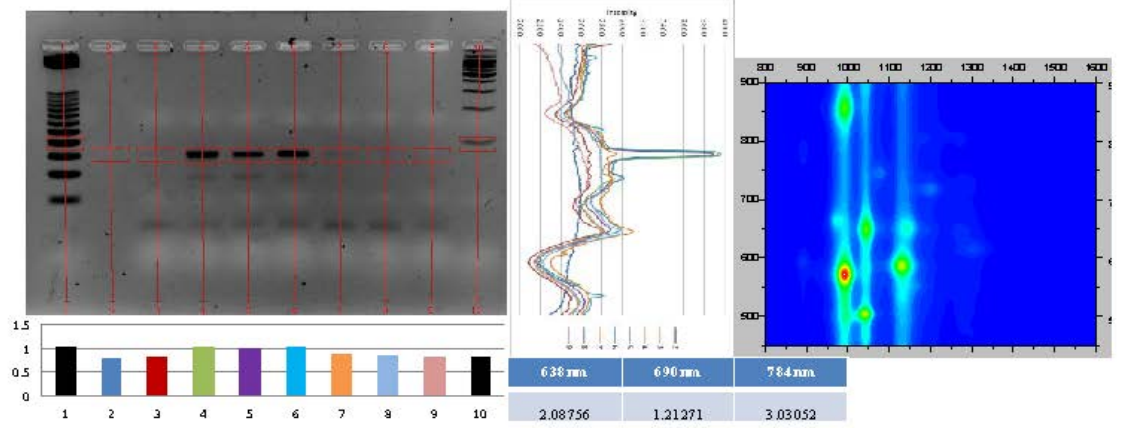

B

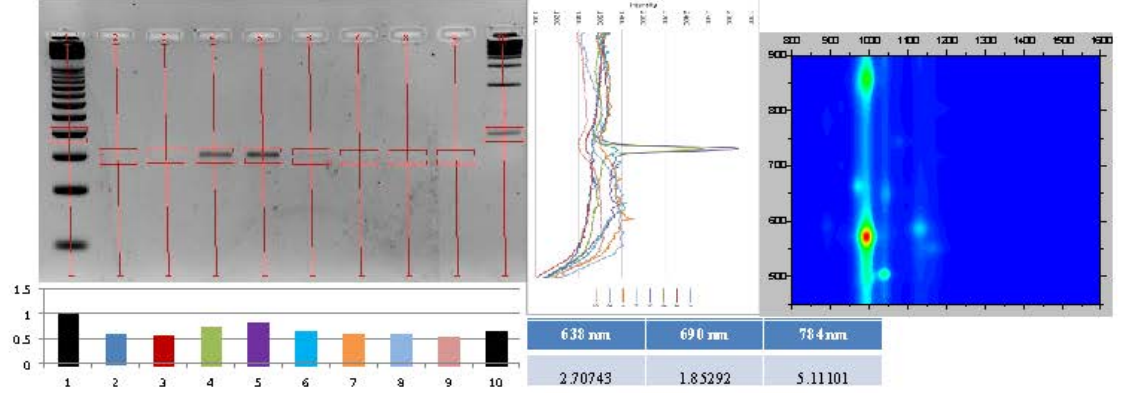

C

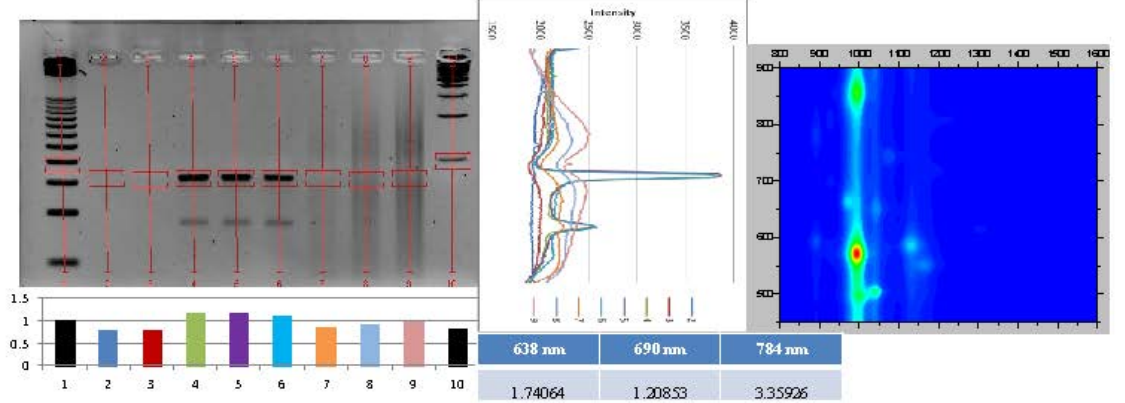

D

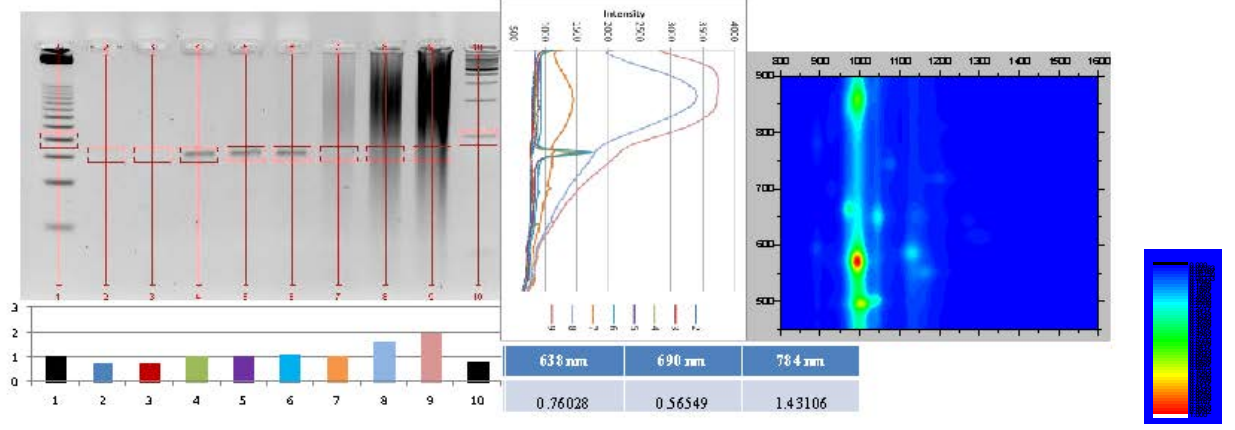

Figure 4.1. (a-d). Agarose gel electrophoresis images and analysis of PCRs with old (4-10 months) DNA:SWCNT samples. For each set, top left is agarose gel image with lanes and quantitized boxes shown, top center is intensity line graph, top right is NS1 synthesized fluorescence intensity plot of DNA:SWCNT samples ( $y$-axis is excitation wavelength, $\mathrm{x}$-axis is emission wavelength), bottom left is bar graph of relative intensity within boxes on gel (normalized to $100 \mathrm{bp}$ marker of 25 bp ladder), bottom center is fluorescence efficiency at each wavelength in NS1 analysis (higher numbers correlate with greater dispersion). Lanes in agarose gel images and relative intensity bar graph: 1. 25 bp ladder (black) 2. empty (dark blue) 3. negative control (red) 4. positive control (green) $5.0 .01 \mathrm{mg} / \mathrm{mL}$ DNA:SWCNT (purple) $6.0 .1 \mathrm{mg} / \mathrm{mL}$ (neon blue) $7.1 \mathrm{mg} / \mathrm{mL}$ (orange) $8.5 \mathrm{mg} / \mathrm{mL}$ (light blue) $9.10 \mathrm{mg} / \mathrm{mL}$ (pink)10. $100 \mathrm{bp}$ ladder (black). Intensity line graph colors correlate with bar graph colors in relative intensity graph. (a) TG15:Mix (b) TG15:(6,5) (c) Salmon:Mix (d)Salmon:(6,5). 

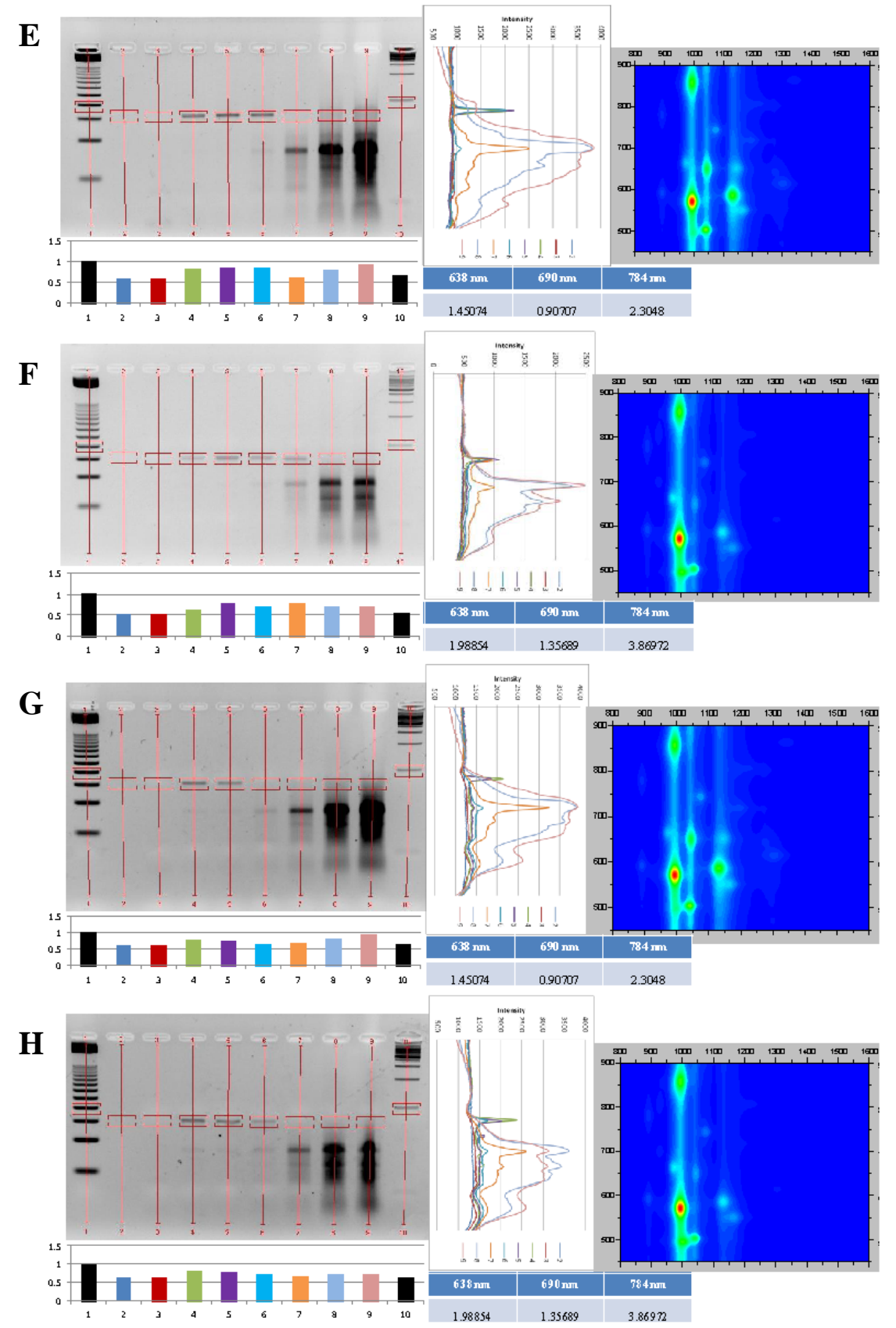

Figure 4.1. (e-h). Agarose gel electrophoresis images and analysis of PCRs with old (4-10 months) DNA:SWCNT samples. For each set, top left is agarose gel image with lanes and quantitized boxes shown, top center is intensity line graph, top right is NS1 synthesized fluorescence intensity plot of DNA:SWCNT samples (y-axis is excitation wavelength, $\mathrm{x}$-axis is emission wavelength), bottom left is bar graph of relative intensity within boxes on gel (normalized to $100 \mathrm{bp}$ marker of 25 bp ladder), bottom center is fluorescence efficiency at each wavelength in NS1 analysis (higher numbers correlate with greater dispersion). Lanes in agarose gel images and relative intensity bar graph: 1. 25 bp ladder (black) 2. empty (dark blue) 3. negative control (red) 4. positive control (green) 5. $0.01 \mathrm{mg} / \mathrm{mL}$ DNA:SWCNT (purple) $6.0 .1 \mathrm{mg} / \mathrm{mL}$ (neon blue) $7.1 \mathrm{mg} / \mathrm{mL}$ (orange) $8.5 \mathrm{mg} / \mathrm{mL}$ (light blue) $9.10 \mathrm{mg} / \mathrm{mL}$ (pink)10. $100 \mathrm{bp}$ ladder (black). Intensity line graph colors correlate with bar graph colors in relative intensity graph. (e) E2 MRE:Mix (f) E2 MRE:(6,5) (g) E2 MRE:Mix, No MRE (h) E2 MRE:(6,5), No MRE. 
DNA:SWCNT and Taq, but it is unlikely to be as strong of an interaction and inhibitory effect as with carboxylic SWCNT.

It is also possible to PCR amplify E2 MRE target which is only present in the reaction wrapped around SWCNT (Figure 4.1g and h). These concentration-dependence patterns follow a similar pattern to reactions performed with the addition of free E2 MRE. This is important in that it shows the ability to directly amplify DNA which is associated with SWCNT.

It is also clear that addition of DNA:SWCNT to PCR reactions has effects on amplicons produced. In both Salmon:SWCNT samples (Figure 4.1c and d intensities), smears are visible in lanes which there is no distinct E2 MRE band. This is likely due to degradation of the genomic DNA present in the sample. In both E2:SWCNT samples amplification of a product just less than 50 bp increases with E2:SWCNT concentration. This is also true for E2:SWCNT PCRs performed with no free E2 MRE in the reaction (Figure 4.1e and f). This is likely due to an excess of the amplification target itself in the reaction. However, in the TG15:SWCNT PCRs, no amplicons appeared that were not present in the control reaction.

\subsubsection{Agarose Gel Analysis of New DNA:SWCNT PCRs}

Analysis of PCRs performed with DNA:SWCNT samples made less than a month before use show amplification in the presence of $0.01 \mathrm{mg} / \mathrm{mL}$ (Figure 4.2 agarose gels). At this concentration, all PCRs performed in the presence of free E2 MRE showed an increase in efficiency of amplification (Figure 4.2 relative intensities). However, in contrast to PCRs with old DNA:SWCNT samples, no amplification of E2 MRE was seen at or above concentrations of $0.1 \mathrm{mg} / \mathrm{mL}$. This interesting result suggests that more well-dispersed SWCNT, as seen in the older samples, is less inhibitory to PCR reactions. This may be because more agglomerated SWCNT hinder the reaction ingredients from encountering each other. This is supported by the 
A

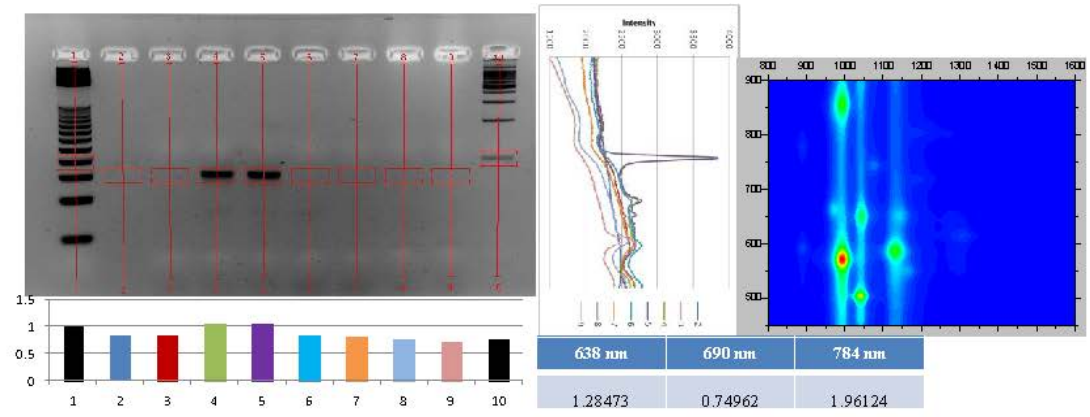

B

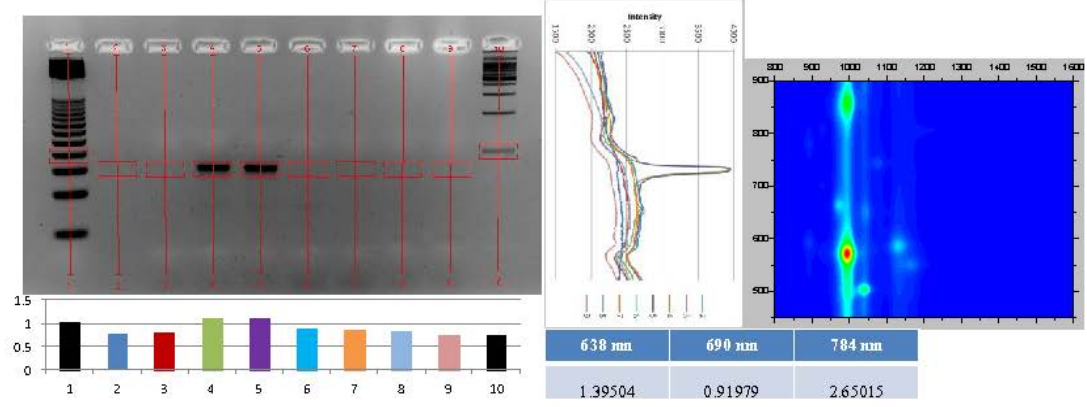

C
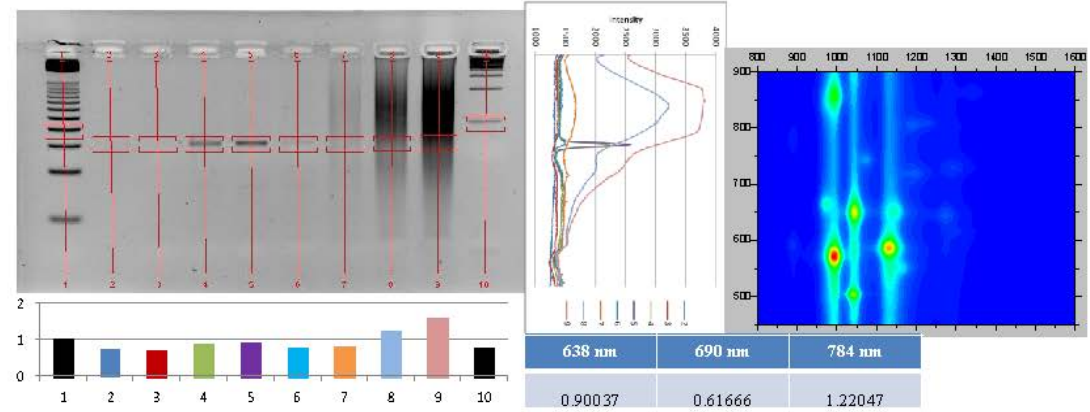

D
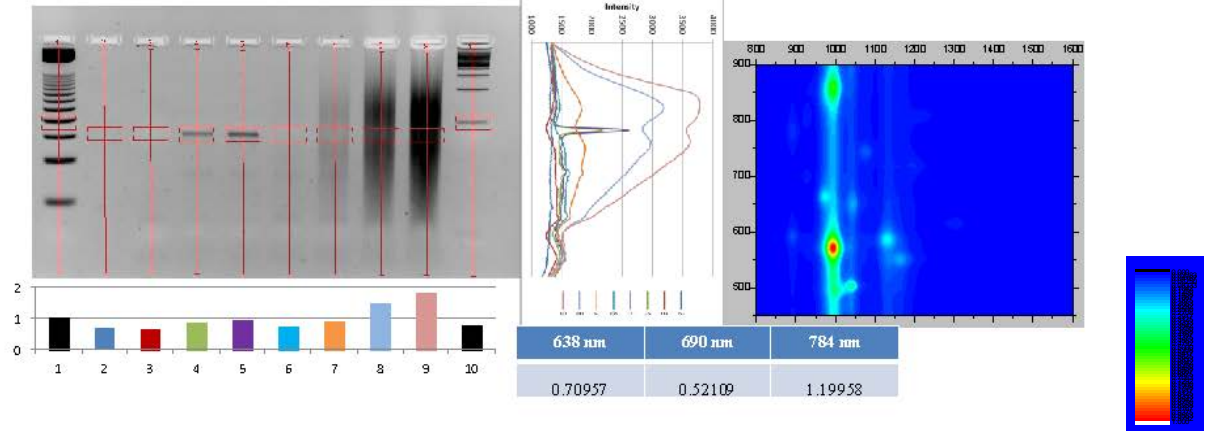

Figure 4.2. (a-d). Agarose gel electrophoresis images and analysis of PCRs with new ( $<1$ month) DNA:SWCNT samples. For each set, top left is agarose gel image with lanes and quantitized boxes shown, top center is intensity line graph, top right is NS1 synthesized fluorescence intensity plot of DNA:SWCNT samples (y-axis is excitation wavelength, $x$-axis is emission wavelength), bottom left is bar graph of relative intensity within boxes on gel (normalized to 100 bp marker of 25 bp ladder), bottom center is fluorescence efficiency at each wavelength in NS1 analysis (higher numbers correlate with greater dispersion). Lanes in agarose gel images and relative intensity bar graph: 1. 25 bp ladder (black) 2. empty (dark blue) 3. negative control (red) 4. positive control (green) $5.0 .01 \mathrm{mg} / \mathrm{mL} \mathrm{DNA:SWCNT}$ (purple) $6.0 .1 \mathrm{mg} / \mathrm{mL}$ (neon blue) $7.1 \mathrm{mg} / \mathrm{mL}$ (orange) $8.5 \mathrm{mg} / \mathrm{mL}$ (light blue) $9.10 \mathrm{mg} / \mathrm{mL}$ (pink)10. $100 \mathrm{bp}$ ladder (black). Intensity line graph colors correlate with bar graph colors in relative intensity graph. (a) TG15:Mix (b) TG15:(6,5) (c) Salmon:Mix (d)Salmon:(6,5). 
E

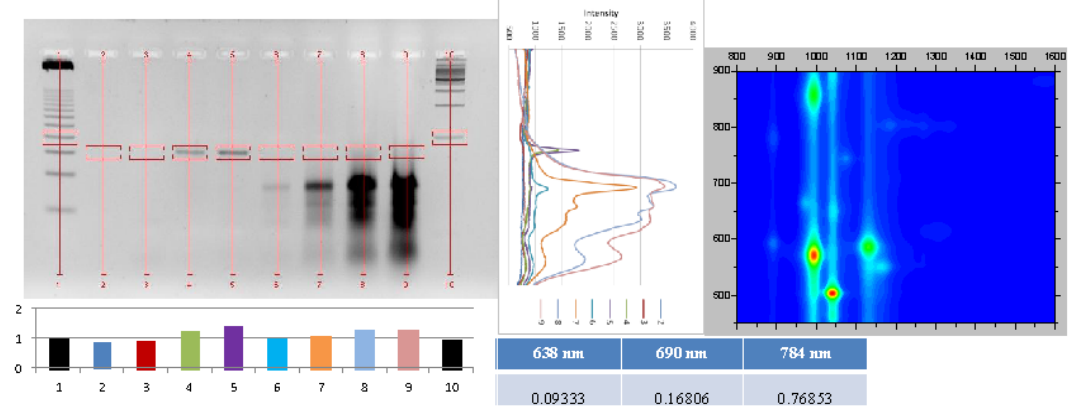

$\mathbf{F}$

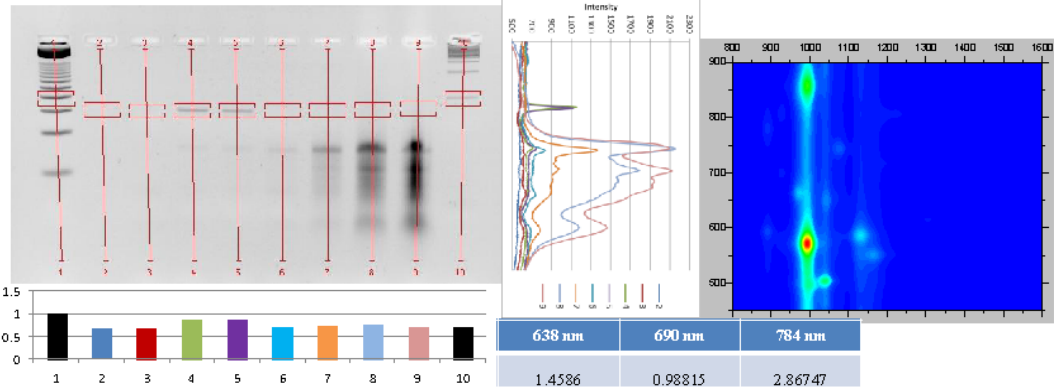

G
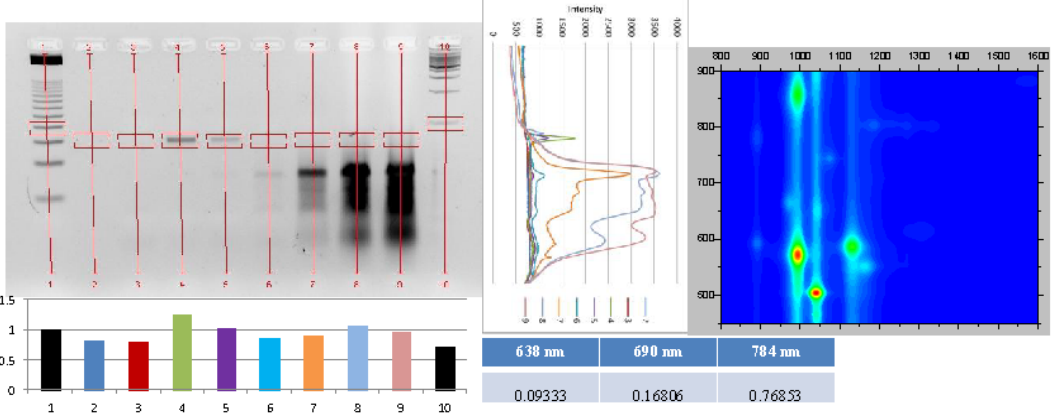

$\mathbf{H}$
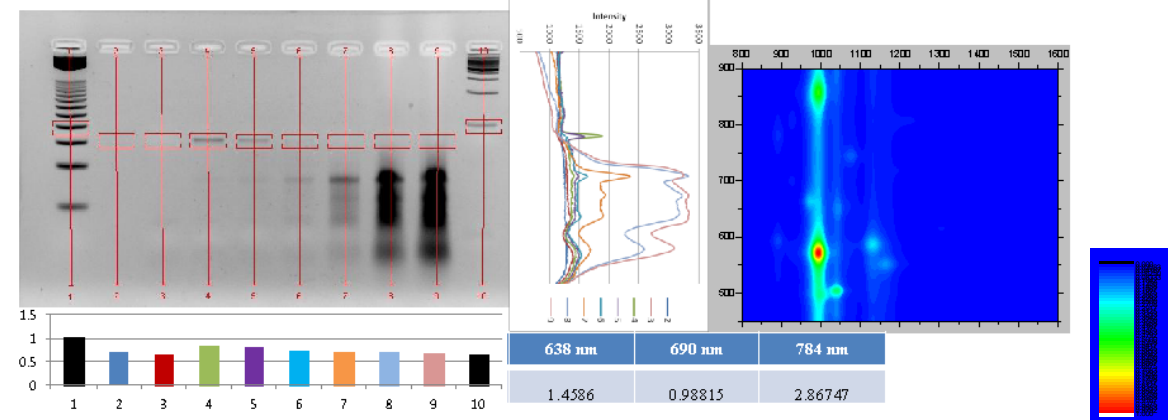

Figure 4.2. (e-h). Agarose gel electrophoresis images and analysis of PCRs with new ( $<1$ month) DNA:SWCNT samples. For each set, top left is agarose gel image with lanes and quantitized boxes shown, top center is intensity line graph, top right is NS1 synthesized fluorescence intensity plot of DNA:SWCNT samples ( $y$-axis is excitation wavelength, $\mathrm{x}$-axis is emission wavelength), bottom left is bar graph of relative intensity within boxes on gel (normalized to $100 \mathrm{bp}$ marker of 25 bp ladder), bottom center is fluorescence efficiency at each wavelength in NS1 analysis (higher numbers correlate with greater dispersion). Lanes in agarose gel images and relative intensity bar graph: 1. 25 bp ladder (black) 2. empty (dark blue) 3. negative control (red) 4. positive control (green) $5.0 .01 \mathrm{mg} / \mathrm{mL}$ DNA:SWCNT (purple) $6.0 .1 \mathrm{mg} / \mathrm{mL}$ (neon blue) $7.1 \mathrm{mg} / \mathrm{mL}$ (orange) $8.5 \mathrm{mg} / \mathrm{mL}$ (light blue) $9.10 \mathrm{mg} / \mathrm{mL}$ (pink)10. $100 \mathrm{bp}$ ladder (black). Intensity line graph colors correlate with bar graph colors in relative intensity graph. (e) E2 MRE:Mix (f) E2 MRE:(6,5) (g) E2 MRE:Mix, No MRE (h) E2 MRE:(6,5), No MRE. 
fact that higher concentrations have an inhibitory effect on PCR efficiency. However, in both old and new samples, there seems to be no difference in PCR efficiency between Mix or (6,5)enriched SWCNT samples. This is in spite of a difference in dispersion based on DNA sequence.

In PCR experiments with new E2:SWCNT samples and absent of free E2 MRE, the MRE was amplified from the SWCNT scaffold (Figure 4.2g and h. Amplification of the 76 bp product is observed in the presence of $0.01 \mathrm{mg} / \mathrm{mL}$ E2:SWCNT, but not at higher concentration. This follows the same pattern seen as PCRs done in the presence of free E2 MRE. There is, however, a reduction in efficiency at this concentration, which contradicts amplification of free E2 MRE at this concentration.

Amplicons produced from PCRs in the presence of new DNA:SWCNT followed the same general pattern as those with old samples. Previous studies have shown SWCNT increase the specificity of long PCR (14 kb) up to the point that the reaction is inhibited (Zhang et al. 2008). The difference in results may be a function of size of the reaction product, or may be due to the method of SWCNT dispersion, which was sonication in water. Another study has shown that different concentrations of carbon nanopowder (CNP) does not affect amplicons produced in a PCR reaction (Zhang et al. 2007). Therefore, the effects are likely due to degradation of genomic DNA or excess target present in the reaction as previously discussed.

\subsection{Conclusions}

This work shows differential inhibitory concentrations for old and new DNA:SWCNT preparations of varying dispersion levels in PCR reactions of 0.1 and $0.01 \mathrm{mg} / \mathrm{mL}$, respectively. This work also shows that it is possible to amplify a DNA sequences that is directly associated 
with SWCNTS through sonication-mediated dispersion. This is important for purification of SWCNT chiralities using DNA as well as use of DNA:SWCNT-based molecular sensors and environmental sample testing applications. 


\section{CHAPTER 5}

Investigation of the Effects of DNA-Dispersed Single-Wall Carbon Nanotubes and Sodium Cholate-Dispersed Single-Wall Carbon Nanotubes on the Green

Algae Chlamydomonas reinhardtii 


\subsection{Introduction}

The increased use of manufactured nanomaterials has led to a need for a better understanding of their potential effects on natural systems. Among these products are carbon nanotubes, which can be represented by sheets of cylindrically-rolled graphene, originally described in 1991 by Iijima (Iijima 1991; Ajayan 1999). The widespread commercialization of single-wall carbon nanotubes (SWCNT) is due to their enormous potential in industrial, biomedical, and electronic sensing applications. This potential is derived from their unique physical, electronic, and optical properties (Wang 2005; Coleman et al. 2006; Huang et al. 2011). The environmental release and transport of SWCNT at all stages of their production and use will lead to environmental concerns (Köhler et al. 2008; Petersen et al. 2011). It is because of their potential that it is necessary to study their interaction with organisms in the environment.

Previous work has shown possible toxic effects of carbon nanomaterials on model organisms. Graphite, C60 fullerene, SWCNT, and multi-wall carbon nanotubes (MWCNT) have been shown to inactivate various Gram-negative and Gram-positive bacteria (Kang et al. 2009). This was shown to increase with incubation times up to four hours. While in the aforementioned work the SWCNT contacted bacteria on a solid support, SWCNT dispersed in solution with various surfactants have shown to be toxic to both bacterial types (Liu et al. 2009). In the model organism Daphnia magna, SWCNT suspended in water with no surfactant were the most toxic among other carbon nano- and bulk- materials (Zhu et al. 2009). These previous works make it clear that SWCNT may have toxic environmental effects, but further exploration of various preparations is necessary.

The unicellular green alga Chlamydomonas reinhardtii has been developed as a model for the effects of nanomaterials on photosynthetic and microorganisms, as well as an indirect 
assessment of ecosystem-level effects (Harris 2001). Work using this model organism has shown toxicity of silver nanoparticles, quantum dots, and titanium dioxide nanoparticles (Navarro et al. 2008; Wang et al. 2008). A subsequent study has shown that this species takes up quantum dots, which cause changes in its transcription profile (Domingos et al. 2011). Additionally, C. reinhardtii has been used in finding toxic effects of SWCNT in solution with no surfactant (Matorin et al. 2010). This work showed a negative effect on growth and photosynthetic output of these algae due to SWCNT aggregates in solution. It specifically tested concentrations of $2 \mu \mathrm{g} / \mathrm{mL}$ for SWCNT and 1 and $2 \mu \mathrm{g} / \mathrm{mL}$ of MWCNT, finding varying negative effects of each. Thus, C. reinhardtii is an established system for studying the direct toxic effects of nanomaterials and for inferring environmental impact of their release.

This study aimed to determine the effects of well-dispersed SWCNT on the model species Chlamydomonas reinhardtii. The system was designed to mimic a stagnant algal growth environment that is similar to natural systems. This design is ideal for well-dispersed SWCNT, however non-dispersed SWCNT would agglomerate and form a precipitate in such a system. Thus, only well-dispersed SWCNT were utilized, mimicking the environmental release of some SWCNT (Köhler et al. 2008). Additionally, agglomerated SWCNT are known to become more well-dispersed over time through interaction with natural organic matter (Lou et al. 2011; Schierz et al. 2012). Therefore, the effects of a wide range of well-dispersed SWCNT in solution with algal growth media on growth and chlorophyll content of green algae was assessed. Furthermore, differences in surfactants used to disperse SWCNTs were explored. Deoxyribonucleic acid (DNA) has been utilized to wrap around SWCNT and disperse them individually in solution (Zheng et al. 2003), while sodium cholate (SC) is a biocompatible surfactant which disperses SWCNT well in solution (Wenseleers et al. 2004; Arnold et al. 2006). 
Herein, DNA-and SC-dispersed SWCNT are characterized by near-infrared (NIR) spectroscopy and their toxicity to $C$. reinhardtii is determined.

\subsection{Methods}

\subsubsection{SWCNT Preparation}

Single-wall carbon nanotubes dispersed by either DNA or SC (DNA:SWCNT or SC:SWCNT) preparations were made similar to as previously described (Zheng et al. 2003; Lahiji et al. 2007). Briefly, 2 mg CoMoCAT SWCNT of mixed chirality (Sigma-Aldrich; St. Louis, MO) were mixed with $2 \mathrm{mg}$ salmon testes genomic DNA or SC in $2 \mathrm{~mL} 1 \mathrm{X}$ phosphatebuffered saline (PBS). This mixture was sonicated on ice for two hours with a Virsonic XL2020 ultrasonic liquid processor (Misonix; Farmingdale, NY) equipped with a $3.2 \mathrm{~mm}$ microtip at approximately $20 \%$ power. The resulting solution was centrifuged at $16,000 \mathrm{Xg}$ for 90 minutes to remove insoluble SWCNT. For DNA:SWCNT samples, the solution was then filtered through a 100,000 molecular weight centrifugal filter (Millipore; Billerica, MA) three times at 4000 RPM for 15 minutes each to remove DNA that did not associate with SWCNTs. The recovered DNA:SWCNT solution was brought to a volume of $500 \mu \mathrm{L}$ with $1 \mathrm{X}$ PBS, while SC:SWCNT samples remained in $2 \mathrm{~mL}$. The concentration of the DNA: or SC:SWCNT samples were obtained by gravimetric analysis of $10 \mu \mathrm{L}$ of the solution weighed on an Ohaus Discovery Microbalance (Ohaus; Parsippany, NJ).

\subsubsection{SWCNT Characterization}

Absorption and NIR fluorescence measurements were performed essentially as previously described (Bachilo et al. 2002; Weisman 2010). Briefly, each SWCNT preparation was diluted to a $1 \%$ solution with Milli-Q water (Millipore; Billerica, MA). This solution was added to a $10 \mathrm{~mm}$ path length cuvette (Sterna Cells; Atascadero, CA) and analyzed on a 
NanoSpectralyzer 1 (NS1) (Applied NanoFluorescence; Houston, TX). The absorbance spectrum was collected from 400 - $1600 \mathrm{~nm}$. Additionally, integrated fluorescent emission spectra were obtained using excitation wavelengths of $638 \mathrm{~nm}, 690 \mathrm{~nm}$, and $784 \mathrm{~nm}$. The resulting spectra were analyzed with ANFSoft (Applied NanoFluorescence; Houston, TX) and prepared in Origin 8 (OriginLab Corporation; Northampton, MA). Fluorescence efficiency measurements, which are fluorescence power collected across the emission spectrum for a single excitation wavelength divided by the absorbance spectrum, are reported as a relative measure of SWCNT dispersion in solution.

\subsubsection{Algae Culture}

Chlamydomonas reinhardtii wild-type strain cc-1690 was obtained from the Chlamydomonas Resource Center (St Paul, MN). Received algae were initially plated onto 1.5\% Sueoka's high salt medium (HSM) agar prepared as previously described (Sueoka 1960). This was used to seed small liquid cultures of HSM for outgrowth, which was kept in a continuous culture of $100 \mathrm{~mL}$ HSM. Every 1-2 weeks, the existing culture was diluted to 10\% in HSM to allow continuous outgrowth. Culture conditions were sustained at $22-25^{\circ} \mathrm{C}$ with continuous ambient light and daily mixing.

\subsubsection{SWCNT Treatments}

Treatments of $C$. reinhardtii in liquid culture with SWCNT preparations or controls were performed in a total volume of $10 \mathrm{~mL}$ in $50 \mathrm{~mL}$ conical tubes with loosened lids. At day zero, 9 mL HSM was seeded with $1 \mathrm{~mL}$ stock algal culture and the appropriate treatment was added. Treatments were: $0,0.1,1,2,10$, or $100 \mu \mathrm{g} / \mathrm{mL}$ DNA:SWCNT or SC:SWCNT, and $100 \mu \mathrm{g} / \mathrm{mL}$ sonicated DNA or SC. Contamination and absorbance controls consisted of $10 \mathrm{~mL}$ of just HSM 
or HSM with $1 \mu \mathrm{g} / \mathrm{mL}$ DNA:SWCNT or SC:SWCNT. Each treatment and control was performed in triplicate and grown for ten days at the above-noted culture conditions.

\subsubsection{Growth Measurements}

Every 24 hours, from day one to day 10, each sample was mixed and $300 \mu \mathrm{L}$ was removed. The optical density of this solution was measured at $750 \mathrm{~nm}$ with a Synergy 2 microplate reader and the software Gen5 1.06 (Biotek US; Winooski, VT). The optical density at $750 \mathrm{~nm}$ was also measured for prepared absorbance controls of HSM with each individual treatment. The optical density of algae was determined by subtracting control from sample optical density. On the tenth day, optical density of Chlorophyll $\beta$ and $\alpha$ at $663 \mathrm{~nm}$ and $645 \mathrm{~nm}$ respectively was obtained. A portion of each sample was centrifuged and resuspended in methanol. This was centrifuged, and optical density of chlorophyll extracted in the liquid phase was measured at $663 \mathrm{~nm}$ and $645 \mathrm{~nm}$ as previously described (Chochois et al. 2010). At the end of treatments, algae were placed onto microscope slides with a cover slip and imaged using a Leica ATC 2000 light microscope (Leica Microsystems; Wetzlar, Germany) and a TCA 5.0 microscope camera (Ample Scientific; Norcross, GA).

\subsection{Results and Discussion}

\subsubsection{SWCNT Preparation and Characterization}

Single-wall carbon nanotubes were dispersed in solution with either DNA or SC. As determined by gravimetric analysis, concentrations of prepared SWCNT solutions ranged from $10 \mu \mathrm{g} / \mu \mathrm{L}$ to $30 \mu \mathrm{g} / \mu \mathrm{L}$. Absorbance and NIR fluorescence spectroscopy was then performed on dilutions of each sample to determine SWCNT chirality composition, diameter, and dispersion in solution (Figures 5.1a and 5.1b). The DNA:SWCNT showed an average SWCNT diameter of $0.83 \mathrm{~nm}$, ranging from 0.6 to $1.4 \mathrm{~nm}$ (Figure 5.1c). Based on an integrated fluorescence 
emission spectrum, the sample of mixed chirality was composed of largely $(6,5),(8,4)$, and $(7,5)$ armchair SWCNT (Figure 5.1e). The SC:SWCNT sample had an average of $1.248 \mathrm{~nm}$, with SWCNT being mostly between 1.2 and $1.3 \mathrm{~nm}$ (Figure 5.1d). The integrated fluorescence emission spectrum of this sample showed a majority of SWCNT being of $(11,7)$ armchair chirality (Figure 5.1f). Additionally, efficiency of fluorescence output of the DNA:SWCNT was much higher than SC:SWCNT, which were negative values (Figures 5.1g and 5.1h). NIR fluorescence is only emitted from monodisperse SWCNT suggesting DNA is more effective at dispersing individual SWCNTs than SC (Zheng et al. 2003; Wenseleers et al. 2004; Liu et al. 2009). DNA gives more monodisperse SWCNT by wrapping around the SWCNT in a helical manner masking the hydrophobic surface of the SWCNT. In contrast, SC forms micelles which disperse SWCNT as bundles. Therefore, fluorescence efficiency and SWCNT diameter of each SWCNT sample confirms that DNA:SWCNT are more well-dispersed than SC:SWCNT.

\subsubsection{Effects of SWCNT and Dispersion Method on Algal Growth}

Chlamydomonas reinhardtii were grown in liquid culture with either DNA:SWCNT, SC:SWCNT, or controls and the culture optical density was measured for ten consecutive days. Results show no normalized optical density for either the high salt medium or $1 \mu \mathrm{g} / \mathrm{mL}$ DNA:SWCNT only controls, meaning no contamination of media or SWCNT sample was present (Figure 5.2). From culture concentrations of $0.1 \mu \mathrm{g} / \mathrm{mL}$ to $100 \mu \mathrm{g} / \mathrm{mL}$, there are no effects of DNA:SWCNT on optical density of the algae. For all cultures, a plateau in algal growth was reached in the sixth day. There is also no difference with treatment of $100 \mu \mathrm{g} / \mathrm{mL}$ sonicated DNA only. 

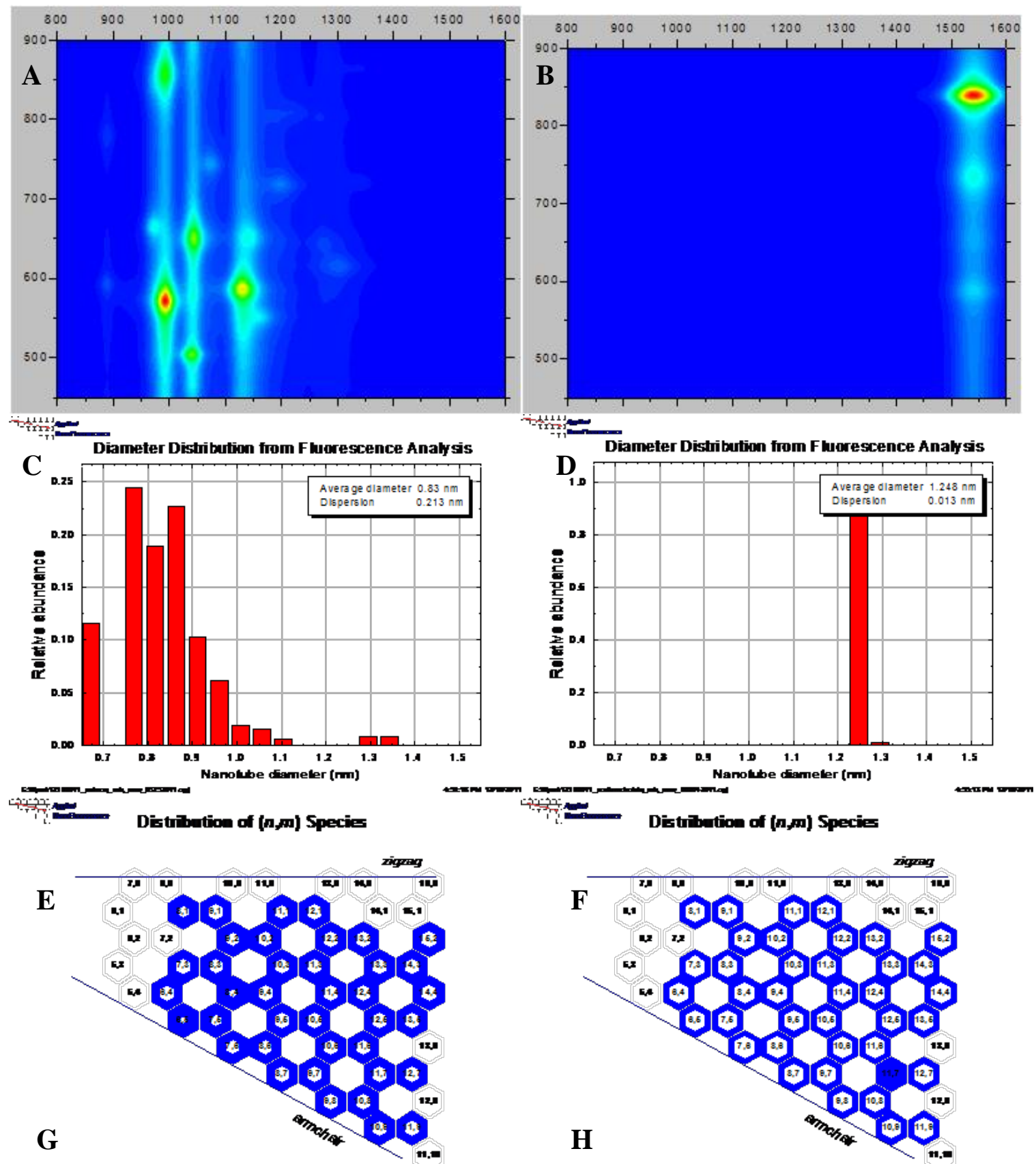

\begin{tabular}{|l|c|c|c||l|l|l|l|}
\hline DNA:SWCNT & $638 \mathrm{~nm}$ & $690 \mathrm{~nm}$ & $784 \mathrm{~nm}$ & SC:SWCNT & $638 \mathrm{~nm}$ & $690 \mathrm{~nm}$ & $784 \mathrm{~nm}$ \\
\hline Efficiency & 1.09507 & 0.75541 & 1.6632 & Efficiency & -0.6205 & -0.23424 & -0.87946 \\
\hline
\end{tabular}

Figure 5.1. Characterization of DNA:SWCNT and SC:SWCNT. (a) and (b) Synthesized fluorescence intensity plot of DNA:SWCNT and SC:SWCNT output respectively. (c) and (d) Calculated density distribution of DNA:SWCNT and SC:SWCNT respectively. (e) and (f) SWCNT chirality distribution of DNA:SWCNT and SC:SWCNT samples respectively. (g) and (h) Fluorescence efficiency measurements at excitation wavelengths of 638, 690, and $784 \mathrm{~nm}$ of DNA:SWCNT and SC:SWCNT respectively. Higher numbers represent better dispersion. 
Results of algae grown in the presence of SC:SWCNT also show no difference in algal growth optical density at $750 \mathrm{~nm}$ due to SC:SWCNT treatment (Figure 5.3). A plateau in growth is evident beginning at day four. There is an initial difference in growth of algae treated with just $100 \mu \mathrm{g} / \mathrm{mL} \mathrm{SC}$ at the plateau, which subsides by the ninth day. At the ninth and tenth day, there is an increase in growth of algae treated with $0.1 \mu \mathrm{g} / \mathrm{mL}$, however this is not significant. Growth of SC only-treated algae plateaus at day 6; however standard deviations also overlap with the control at these days suggesting no true difference.

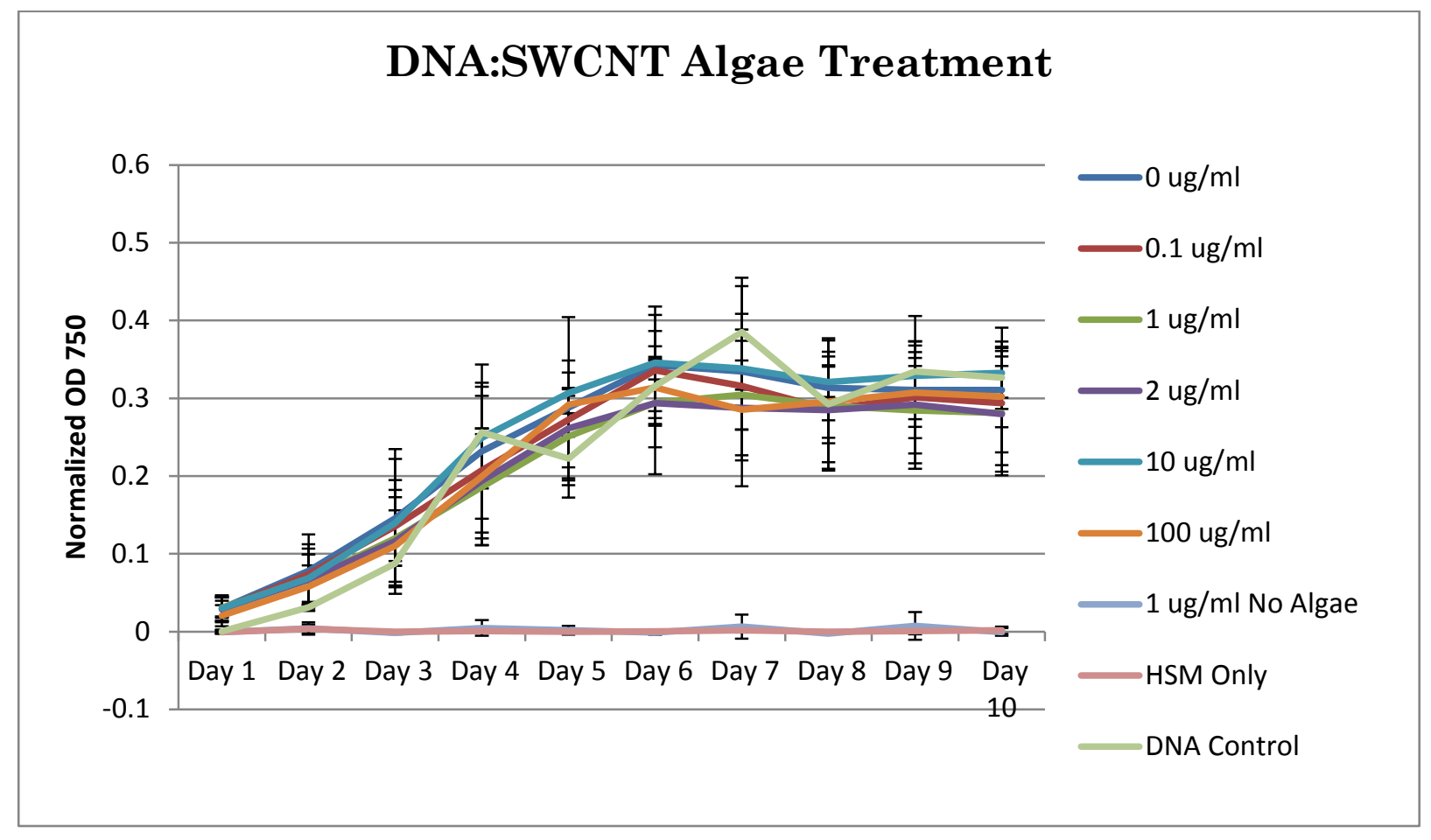

Figure 5.2. Ten day growth curve of Chlamydomonas reinhardtii with DNA:SWCNT treatments or controls. Treatments were $0,0.1,1,2,10$, or $100 \mu \mathrm{g} / \mathrm{mL}$, DNA:SWCNT only control, media only control (HSM), or $100 \mu \mathrm{g} / \mathrm{mL}$ DNA control. Errors bars for each point represent (+/-) standard deviation.

After the tenth day, extraction of chlorophyll and absorbance measurements at $663 \mathrm{~nm}$ and $645 \mathrm{~nm}$ gave chlorophyll content measurements of the algal cultures. At $663 \mathrm{~nm}$, absorbance of chlorophyll $\beta$ showed no difference between either DNA:SWCNT or SC:SWCNT and their control (Figure 5.4). Additionally, there is no difference between DNA and SC 
dispersed SWCNT at any treatment concentration. There is a decrease in SC-only treated algae, however. This may be due to some mitigation by SWCNT being complexed with SC.

Chlorophyll $\alpha$ absorbance at $645 \mathrm{~nm}$ showed similar results (Figure 5.5). There is no difference between controls and treatments of DNA:SWCNT and SC:SWCNT at any treatment level.

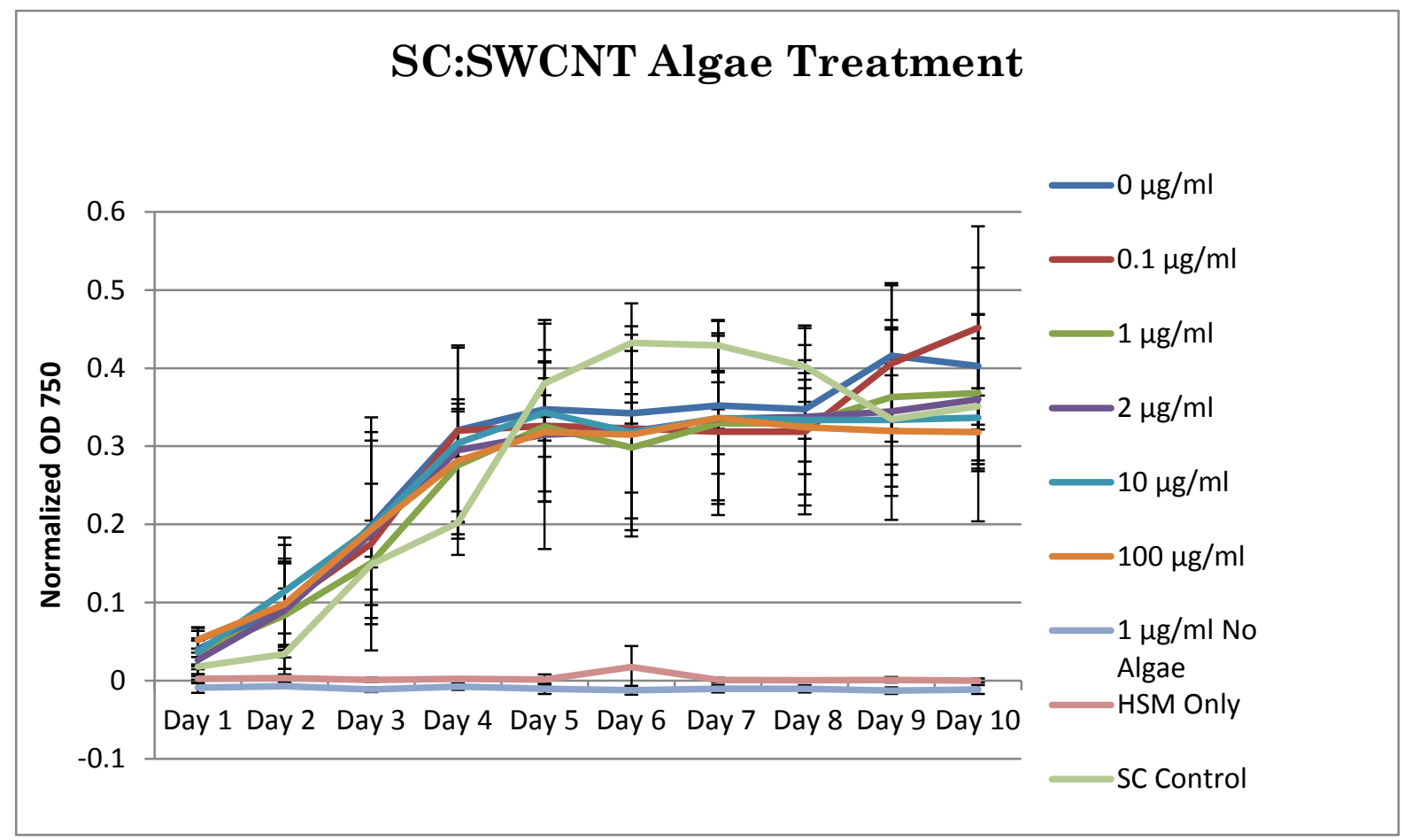

Figure 5.3. Ten day growth curve of Chlamydomonas reinhardtii with SC:SWCNT treatments or controls. Treatments were $0,0.1,1,2,10$, or $100 \mu \mathrm{g} / \mathrm{mL}$, SC:SWCNT only control, media only control (HSM), or $100 \mu \mathrm{g} / \mathrm{mL}$ SC control. Errors bars for each point represent (+/-) standard deviation.

There seems to be no difference in Chlamydomonas reinhardtii growth or chlorophyll output for DNA:SWCNT or SC:SWCNT treatment relative to control. This is in opposition to previously-reported data showing negative growth effects of SWCNT. Previous findings using 2 $\mu \mathrm{g} / \mathrm{mL}$ SWCNT with no surfactant showed a negative effect on growth of algae up to five days (Matorin et al. 2010). This report also showed clear evidence of algal association with SWCNT agglomerations. Non-dispersed SWCNT have also shown to be toxic to bacteria (Kang et al. 2009). Furthermore, a $\mathrm{LC}_{50}$ of $2.4 \mu \mathrm{g} / \mathrm{mL}$ has been obtained with Daphnia magna. This level 
and almost 50 times greater caused no growth effects in the current study. While the stagnant growth conditions used in this study were not conducive to using non-dispersed SWCNT due to hydrophobic precipitation, the previously-reported studies are a comparative benchmark for their toxic effects. The use of well-dispersed SWCNT is environmentally relevant as agglomerations are known to associate with organic matter and become more well-dispersed over time (Lou et al. 2011; Schierz et al. 2012). The extensive studies reported herein suggest there is no negative effect of well-dispersed SWCNT on algal growth.

The reported toxic effect of SWCNT may be due to agglomeration and shading, which was mitigated in this study with more effective dispersion of the SWCNTs. It has been reported that SWCNT toxic effects on the green algae Pseudokirchneriella subcapitata were lost with higher concentrations of the surfactant Gum Arabic (Youn et al. 2011). Another study specifically investigating the effects of agglomeration and shading found that SWCNT partially dispersed by natural organic matter had $\mathrm{EC}_{50}$ values of $1.8 \mu \mathrm{g} / \mathrm{mL}$ and $20 \mu \mathrm{g} / \mathrm{mL}$ on the algae Chlorella vulgaris and P. subcapitata (Schwab et al. 2011). That work suggests that toxic effects may be due to indirect effects on local growing conditions of algae within SWCNT agglomerations, such as competition for resources and reduced light availability.

In the current study, agglomeration and shading by SWCNT did not seem to be as prevalent. No agglomeration of DNA:SWCNT samples were seen either in culture or in micrographs (Figure 5.6). At the highest level of SC:SWCNT treatment, $100 \mu \mathrm{g} / \mathrm{mL}$, there was some visible agglomeration in culture and under the microscope, but this was not extensive nor was it found at any lower levels. However, microscopic examination shows only a small portion of algae are agglomerated with SC:SWCNT, not nearly as prevalent as with the P. subcapitata study. Furthermore, growth at the $100 \mu \mathrm{g} / \mathrm{mL}$ treatment level was not affected. 


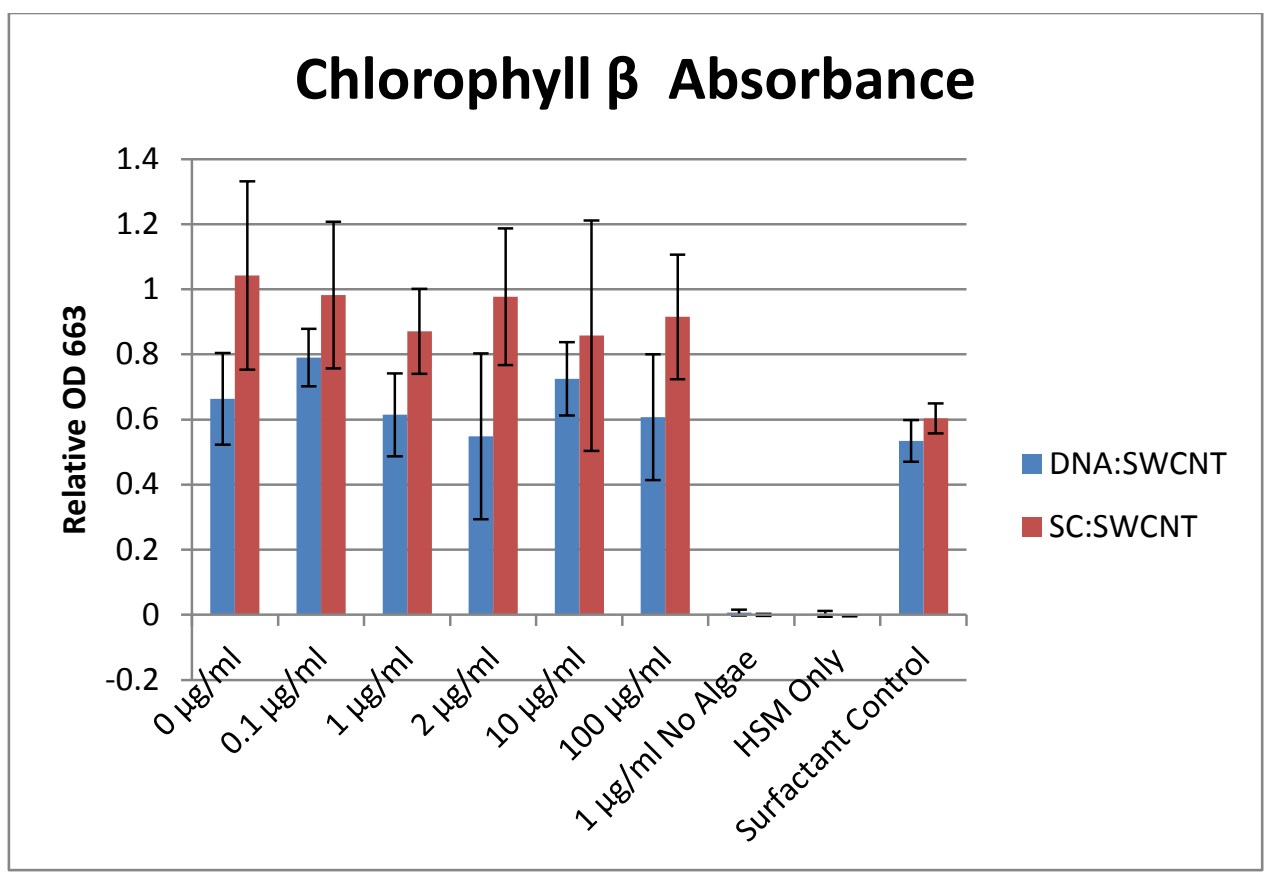

Figure 5.4. Extracted Chlorophyll $\beta$ content at OD 663 for DNA:SWCNT and SC:SWCNT treatments. Treatments are $0,0.1,1,2,10$, or $100 \mu \mathrm{g} / \mathrm{mL}$, DNA:SWCNT or SC:SWCNT only

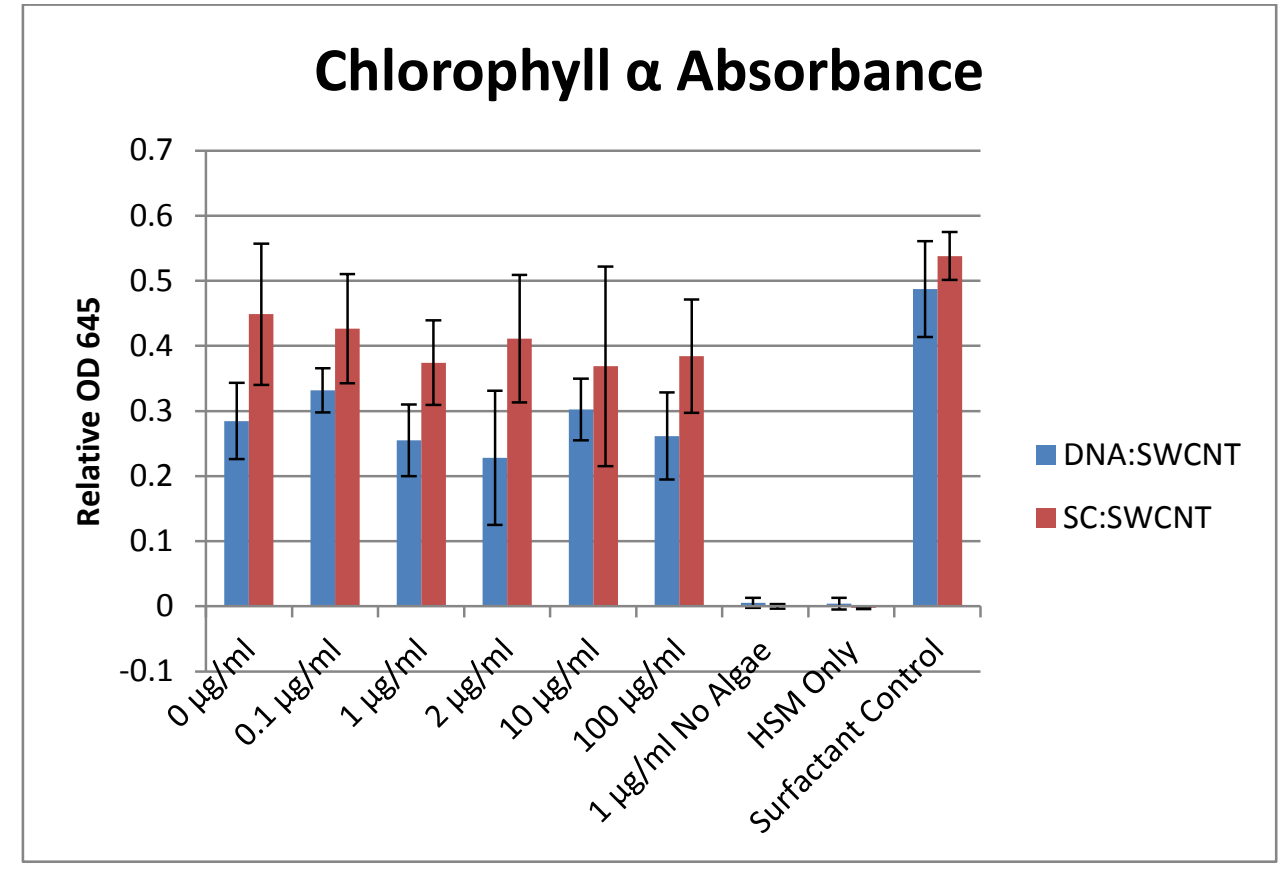

Figure 5.5. Extracted Chlorophyll $\alpha$ content at OD 645 for DNA:SWCNT and SC:SWCNT treatments. Treatments are $0,0.1,1,2,10$, or $100 \mu \mathrm{g} / \mathrm{mL}$, DNA:SWCNT or SC:SWCNT only control, media only control (HSM), or $100 \mu \mathrm{g} / \mathrm{mL}$ DNA or SC control. Error bars for each point represent (+/-) standard deviation. 
Additionally, the chosen strain can grow in the presence of nitrogen in the dark and therefore may not be affected by SWCNT shading effects. Results of chlorophyll content measurements however show no difference due to SC:SWCNT at any level. Future work may determine possible effects on chlorophyll efficiency due to agglomeration and shading. Hence, the lack of toxic SWCNT effects is likely due to the well-dispersed nature of DNA:SWCNT and SC:SWCNT. Indeed, work with SWCNT well-dispersed in various surfactants on rat liver cells and human lung fibroblasts showed no toxic effects (Alpatova et al. 2010; Wang et al. 2010). Furthermore, DNA:SWCNT and SC:SWCNT showed no toxic effect on human astrocytoma cells (Dong et al. 2008). A lack in difference of growth between DNA:SWCNT and SC:SWCNT, even though DNA:SWCNT are more well-dispersed, may be because SC:SWCNT do not agglomerate to the point of causing a wide-scale effect on growth.

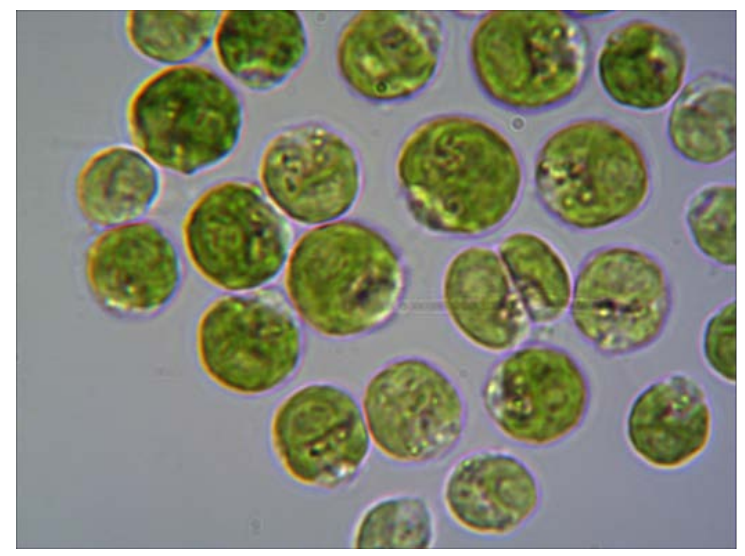

Figure 5.6. Micrograph of algae grown with SWCNT. Representative image of Chlamydomonas reinhardtii after ten days in culture with $100 \mu \mathrm{g} / \mathrm{mL}$ DNA:SWCNT. Algae appear healthy and no SWCNT agglomerations are visible.

A detraction from this hypothesis is a study showing that SWCNT act as 'nano darts'. In this, well-dispersed SC:SWCNT effectively punctured bacterial membranes (Liu et al. 2009). This work states that softer bacterial membranes were more susceptible to puncturing by SWCNT, and therefore may not be a factor with the prominent glycoprotein cell wall of $C$. reinhardtii (Harris 2001). It was also noted that a higher rate of bacterial death was obtained by 
faster shaking speeds with SWCNT. This was not a factor here as cultures were only mixed once a day. Future studies may be directed to investigate increased shaking speeds of DNA:SWCNT and SC:SWCNT treated cultures. The presented work does, however, agree with other toxicological studies using DNA:SWCNT and SC:SWCNT to treat human cells (Dong et al. 2008). It is therefore unlikely that SWCNT punctured the cell wall of the algae or had any other negative effects. Future work may further investigate the nature of the interaction seen in SC:SWCNT agglomerations and investigate the ability of monodisperse SWCNT to penetrate algal cell walls.

\subsection{Conclusions}

This study presents findings that SWCNT well-dispersed with DNA or SC does not have an effect on growth or chlorophyll content of Chlamydomonas reinhardtii at concentrations of 0.1-100 $\mu \mathrm{g} / \mathrm{mL}$. This is in opposition to previous work that has shown SWCNT can be toxic to algae, bacteria, or other model organisms. However, this supports other research that suggests there is no toxic effect of well-dispersed SWCNT on model organisms or mammalian cells. Thus, increasingly widespread use of SWCNT may have toxic environmental impacts if the materials are poorly dispersed in solution. However, well-dispersed SWCNT, such as those with DNA or SC, may have minimal environmental consequences. 


\section{Appendix A}

In Vitro Selection of Antibody Fragment Molecular Recognition Elements for Prostate Cancer Cells 


\section{A.1 Introduction}

Prostate cancer is the most-diagnosed non-skin cancer in the United States, with an estimated 241,740 new diagnoses in 2012 alone (Siegel et al. 2012). It is the second leading cause of cancer-related deaths among males in the U.S., with an estimated 28,170 mortalities in 2012 (Siegel et al. 2012). Approximately one in six U.S. men will get prostate cancer in their lifetime, with one in 30 dying from the disease (Ries et al. 1997). It is therefore important to develop new diagnostic and therapeutic approaches for clinical prostate cancer treatment.

Currently, prostate cancer diagnosis has shifted away from the standard of care: prostate specific antigen (PSA) blood levels. Initially discovered in 1979, serum PSA levels were shown to be higher in men with prostate cancer than those with normal prostates (Wang et al. 1979; Kuriyama et al. 1980). After U.S. Food \& Drug Administration approval for prostate cancer detection in 1986, there was an initial increase in number of prostate cancer-related deaths, largely due to more diagnoses (Catalona et al. 1991; Baade et al. 2004). This mortality rate began to decrease due to better awareness, however, as PSA had become the standard bearer in cancer biomarkers (Baade et al. 2004).

In spite of this promise, PSA testing is no longer recommended for use by the United States Preventative Services Task Force (USPSTF) (Chou et al. 2011; Lin et al. 2011; Moyer 2012). This is because serum PSA levels are raised not only due to cancerous prostates, but due to benign prostatic conditions such as prostatic intraepithelial neoplasia (PIN), benign prostate hyperplasia (BPH), and prostatitis (Woolf 1995). Indeed, studies have shown the specificity of the PSA test in prostate cancer diagnosis to be just $24 \%$, meaning there is a $76 \%$ over-diagnosis rate (Thompson et al. 2006). Calculations have shown that PSA screening will prevent just one prostate cancer-related death in greater than 1000 men (Moyer 2012). For that small number is 
does help, it is estimated to cause many unnecessary biopsies, treatments, and individual stress (Carlsson et al. 2012; Moyer 2012). For that reason, the USPSTF officially gave it a grade 'D' in 2012; however some primary care physicians continue to use the test due to its high sensitivity (Barry 2009; Moyer 2012; Pollack et al. 2012).

The promise of PSA, despite its pitfalls, has led to a great deal of research in identifying other diagnostic mechanisms. There have been many biomarkers identified that may have some diagnostic potential (Herness and Naz 2001; Williams and Naz 2010). Many of these, however, do not differentiate between prostate cancer and other prostatic diseases, similar to PSA. Others are expressed in other tissues and are not useful for identification of prostate cancer. The moststudied among these is prostate-specific membrane antigen (PSMA), which has entered into the clinic as a diagnostic and therapeutic tool (Horoszewicz et al. 1987). PSMA, however, is expressed in other non-prostatic normal and malignant tissues (Maraj et al. 1998; Renneberg et al. 1999). Thus, clinical prostate cancer diagnosis relies on digital rectal exams, which can miss tumors, relies on diagnosis after palpable tumors have formed, and misses more prostate tumors than PSA screening (Catalona et al. 1994). Additionally, biopsies are performed, which are invasive and have inherent infection risks (Rodriguez and Terris 1998). Therefore, it is necessary to develop a minimally invasive diagnostic molecular test for the early and specific detection of prostate cancer.

In addition to non-specific diagnostics, current treatments for prostate cancer are partially effective, but have many unwanted side effects. Early-stage, localized prostate cancer is typically either managed with watchful waiting or treated with prostatectomy, radiation therapy, androgen deprivation, cryotherapy, or ultrasonogrpahy (Chou et al. 2011). Long-term studies have found that radical prostatectomy confers a significant decrease in mortality compared to 
watchful waiting, from $21 \%$ to $15 \%$ in one trial (Wong et al. 2006; Albertsen et al. 2007; BillAxelson et al. 2008; Bill-Axelson et al. 2011). In total, radiotherapy, including external beam radiation therapy and internal brachytherapy, significantly reduces prostate cancer-related mortality compared to no treatment (Tewari et al. 2007; Zhou et al. 2009; Ladjevardi et al. 2010; Stattin et al. 2010). Conversely, androgen deprivation therapy is actually associated with increased risk of mortality, however one study found a slight decrease after a seven year followup period (Lu-Yao et al. 2008; Zhou et al. 2009).

Each of the localized prostate cancer treatment modalities is associated with multiple side-effects. These include urinary incontinence, erectile dysfunction, and quality of life, among others (Chou et al. 2011). In fact, depending on the treatment, a 14-33\% higher proportion of men, compared to watchful waiting, suffered from erectile dysfunction and a $20 \%$ higher proportion of men had urinary incontinence (Chou et al. 2011). For prostatectomy, operationrelated mortality and cardiac events are possible (Yao and Lu-Yao 1999). Additionally, recurrence is possible with all of these treatments. This can be in a more aggressive, androgenindependent form, or other hard-to-treat forms (Feldman and Feldman 2001). For many nonlocalized cancers, chemotherapy is also used. This is in spite of the fact that many advanced, hormone-refractory prostate cancers are difficult to treat with chemotherapeutics (Yagoda and Petrylak 2006). For prostate cancer, historically 5-fluorouracil (5-FU) was used and had fewer side effects compared to those used today (Yagoda and Petrylak 2006). Currently, docetaxel is the first-line chemotherapeutic, with mitoxantrone sometimes being used in that role based on side effects (Pienta 2001; Pienta and Smith 2005). These are often used with combinations of other agents as well (Pienta and Smith 2005). However, whether alone or in combination, these cause many side effects due to non-specific action. A 70\% response rate has been shown for 
patients on docetaxel, however as many as $70 \%$ of all patients also experienced neutropenia (Extra et al. 1993). Other main side effects include thrombosis, fatigue, hyperglycemia, nausea, vomiting, and others (Pienta and Smith 2005). Clearly, these drugs can be effective, however more specific therapeutics would be more effective and have fewer side effects.

It is possible to utilize molecular targeting to diagnose and specifically deliver therapeutics to prostate cancer. Identification of a Molecular Recognition Element (MRE) is achieved through the process of Selective Evolution of Ligands by EXponential Enrichment (SELEX), also known as in vitro selection (Ellington and Szostak 1990; Tuerk and Gold 1990). This process utilizes a large library of random molecules which are screened for their ability to bind to a target of interest. Those that do not bind to the target are removed, and those that do are amplified. Amplified molecules are then subjected to closely-related negative targets to which binding is not desired. Molecules that do not bind to this target are amplified, completing a round of selection. Rounds continue until one or a few strongly-binding and specific molecules are selected. This process has been adapted for use in identifying molecules that bind to wholecell targets (Morris et al. 1998; Guo et al. 2008). It has also been adapted to incorporate a protein library displayed on various microbiological host surfaces, including yeast (Huse et al. 1989; Gunneriusson et al. 1996; Boder and Wittrup 1997). It is therefore possible to combine these methodologies to identify protein MREs from a yeast-displayed library which bind to the surface of prostate cancer cells.

The work described here utilized a novel variation of SELEX called Decoy-SELEX. This scheme enriches the initial library for molecules which bind to the target of interest similar to SELEX. It focuses, however, on stringent negative selections using targets to which the MRE should not bind. This work has identified MREs which bind to a prostate cancer cell line, and 
not to various benign prostate cells lines. It utilized a human non-immune single-chain Fragment variable (scFv) library displayed on the surface of Sacchoromyces cerevisiae. The obtained MREs will be useful for both specific diagnosis and treatment of prostate cancer.

\section{A.2 Methods}

The Decoy-SELEX process was used in order to obtain a prostate cancer cell-specific MRE (Figure A.1). A library of non-immune human single-chain Fragment variable (scFv) antibody fragments was displayed on the surface of yeast. Seven rounds of selection were completed, enriching for those scFvs which bound to the androgen-dependent prostate cancer cell line LNCaP and subtracting those that bound to the benign prostate cell lines HGPIN, BPH1, and BHPrE1, as well as the protein PSMA (Table A.1).

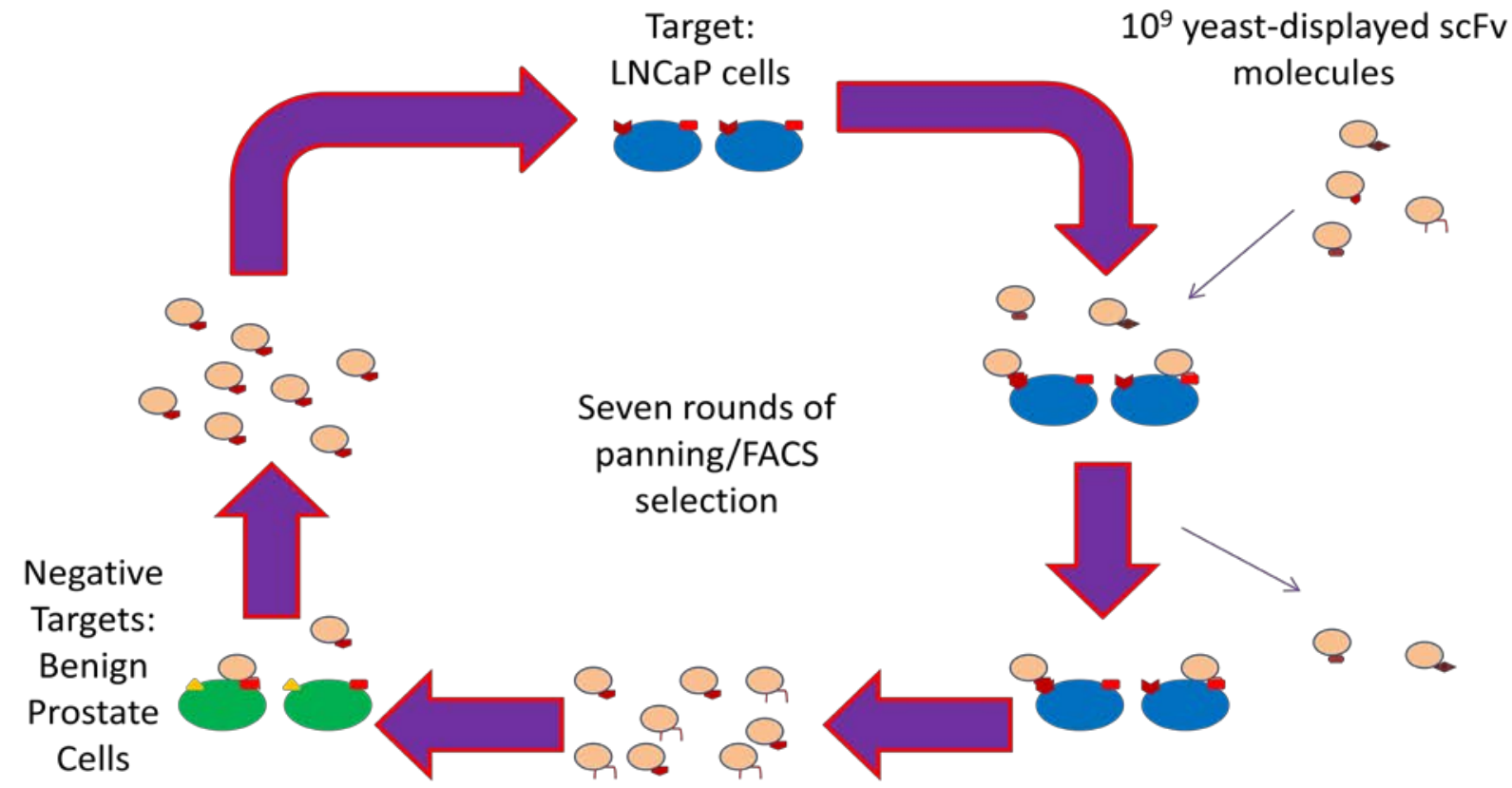

Figure A.1. Scheme depicting the Decoy-SELEX process. A library of $10^{9}$ yeast-displayed scFv molecules are incubated with the target LNCaP cell line. Those that bind are amplified and incubated with negative targets. Those that do not bind the negative targets are amplified and subjected to additional rounds of in vitro selection. 


\section{A.2.1 Cell Culture and Materials}

In order to obtain a prostate cancer cell-specific MRE, prostatic cell lines were used. For general maintenance, each line was passaged every 5-7 days in a T75 cell culture dish with media changed every 2-3 days. The cells were grown in a $37^{\circ} \mathrm{C}$ incubator with $5 \%$ carbon dioxide and humidity. The androgen-dependent prostate cancer cell line LNCaP was obtained from the American Type Culture Collection (ATCC) (Manassas, VA) and cultured in RPMI 1640 with L-Glutamine and 25 mM HEPES (Cellgro; Manassas, VA) and 10\% Fetal Bovine Serum (FBS) (Fisher Scientific; Pittsburgh, PA) and 1X antibiotic/antimycotic mixture (ab/am) (Cellgro) (Horoszewicz et al. 1983). The HPV-18-immortalized High Grade Prostatic Intraepithelial Neoplasia (HGPIN) cell line was a generous gift from Dr. Mark Stearns (Drexel University; Philadelphia, PA) and was cultured in Defined KSFM (Gibco; Grand Island, NY) with 5\% FBS and 1X ab/am (Wang et al. 1999). The SV40T-immortalized Benign Prostate Hyperplasia (BPH-1) cell line was a generous gift from Dr. Simon Hayward (Vanderbilt University; Nashville, TN) and was cultured in RPMI-1640 with L-Glutamine and 25 mM HEPES and 10\% FBS and $1 \mathrm{X}$ ab/am (Hayward et al. 1995). The spontaneously-immortalized intermediate prostate stem cell line BHPrE1 was also obtained as a generous gift from Dr. Simon Hayward (Vanderbilt University) and cultured in DMEM/F12 (Cellgro) supplemented with 5\% FBS, $1 \mathrm{X}$ ab/am, 1\% insulin/transferrin/selenium (Gibco), 0.4\% bovine pituitary extract (Sigma; St. Louis, MO), $5 \mathrm{ng} / \mathrm{mL}$ epidermal growth factor (Gemini Bio-Products; West Sacramento, CA), and $1 \mathrm{X}$ ab/am (Jiang et al. 2009). The androgen-independent DU-145 prostate cancer cell line was obtained from ATCC and cultured in EMEM (Cellgro) with 10\% FBS and 1X ab/am (Stone et al. 1978). The androgen-independent prostate cancer cell line PC-3 was also obtained from ATCC and cultured in F12K media (Cellgro) with 10\% FBS and 
Table A.1. Decoy-SELEX scheme for identification of prostate cancer cell-specific MREs.

\begin{tabular}{|c|c|c|c|c|c|c|c|}
\hline $\begin{array}{l}\text { Selection } \\
\text { Round }\end{array}$ & Target & \begin{tabular}{|l} 
Yeast \\
Number
\end{tabular} & $\begin{array}{l}\text { \% Yeast } \\
\text { Expression }\end{array}$ & \begin{tabular}{|l|} 
Human \\
Cell \\
Number \\
\end{tabular} & $\begin{array}{l}\text { Incubation } \\
\text { Time } \\
\end{array}$ & Volume & $\begin{array}{l}\text { Separation } \\
\text { Method }\end{array}$ \\
\hline $1(+)$ & LNCaP & $1 \times 10^{10}$ & 34.2 & $\sim 8 \times 10^{6}$ & 3 hours & $15 \mathrm{~mL}$ & Panning \\
\hline \multirow{3}{*}{$1(-)$} & HGPIN & \multirow{3}{*}{$1 \times 10^{10}$} & \multirow{3}{*}{38.5} & $\sim 7 \times 10^{6}$ & 30 minutes & \multirow{3}{*}{$15 \mathrm{~mL}$} & \multirow{3}{*}{ Panning } \\
\hline & BPH-1 & & & \begin{tabular}{|l}
$\sim 9 \times 10^{6}$ \\
\end{tabular} & 30 minutes & & \\
\hline & BHPrE1 & & & $\sim 7.5 \times 10^{6}$ & 30 minutes & & \\
\hline $2(+)$ & LNCaP & $1 \times 10^{10}$ & 7.04 & $\sim 9 \times 10^{6}$ & 2 hours & $15 \mathrm{~mL}$ & Panning \\
\hline \multirow{3}{*}{$2(-)$} & HGPIN & \multirow{3}{*}{$1 \times 10^{10}$} & \multirow{3}{*}{6.34} & $\sim 8.5 \times 10^{6}$ & 1 hour & \multirow{3}{*}{$15 \mathrm{~mL}$} & \multirow{3}{*}{ Panning } \\
\hline & BPH-1 & & & $\sim 9.5 \times 10^{6}$ & 1 hour & & \\
\hline & BHPrE1 & & & \begin{tabular}{|l|}
$\sim 8 \times 10^{6}$ \\
\end{tabular} & 1 hour & & \\
\hline $3(+)$ & LNCaP & $1 \times 10^{10}$ & 8.85 & $\sim 9 \times 10^{6}$ & 1 hour & $15 \mathrm{~mL}$ & Panning \\
\hline \multirow{3}{*}{$3(-)$} & HGPIN & \multirow{3}{*}{$1 \times 10^{10}$} & \multirow{3}{*}{6.7} & $\sim 7 \times 10^{6}$ & 2 hours & \multirow{3}{*}{$15 \mathrm{~mL}$} & \multirow{3}{*}{ Panning } \\
\hline & BPH-1 & & & $\sim 9 \times 10^{6}$ & 2 hours & & \\
\hline & BHPrE1 & & & \begin{tabular}{|l|l|}
$\sim 8 \times 10^{6}$ \\
\end{tabular} & 2 hours & & \\
\hline $4(+)$ & LNCaP & $1 \times 10^{7}$ & 29.65 & $1 \times 10^{6}$ & 30 minutes & $2.5 \mathrm{~mL}$ & FACS \\
\hline $4(-) \mathrm{a}$ & HGPIN & $1 \times 10^{7}$ & 6.31 & $1 \times 10^{6}$ & 30 minutes & $1.5 \mathrm{~mL}$ & FACS \\
\hline $4(-) \mathrm{b}$ & BPH-1 & $1 \times 10^{7}$ & 7.65 & $1 \times 10^{6}$ & 30 minutes & $2 \mathrm{~mL}$ & FACS \\
\hline $4(-) c$ & BHPrE1 & $1 \times 10^{7}$ & 4.78 & $1 \times 10^{6}$ & 30 minutes & $1.5 \mathrm{~mL}$ & FACS \\
\hline $4(-) \mathrm{d}$ & PSMA* & $1 \times 10^{7}$ & 17.3 & $\begin{array}{l}1 \mathrm{nmol} \\
\text { protein }\end{array}$ & 30 minutes & $1 \mathrm{~mL}$ & FACS \\
\hline $5(+)$ & LNCaP & $1 \times 10^{7}$ & $\begin{array}{l}24.02 \\
(\mathrm{Lo}) / 21.72 \\
(\mathrm{Hi})\end{array}$ & $1 \times 10^{6}$ & 30 minutes & $5 \mathrm{~mL}$ & FACS \\
\hline $6(+)$ & LNCaP & $1 \times 10^{7}$ & $21.2 / 19.36$ & $1 \times 10^{5}$ & 30 minutes & $2.75 \mathrm{~mL}$ & FACS \\
\hline $6(-) a$ & HGPIN & $1 \times 10^{7}$ & $16.73 / 13.5$ & $1 \times 10^{6}$ & 30 minutes & $2.5 \mathrm{~mL}$ & FACS \\
\hline $6(-) b$ & BPH-1 & $1 \times 10^{7}$ & $7.86 / 11.92$ & $1 \times 10^{6}$ & 30 minutes & $2.5 \mathrm{~mL}$ & FACS \\
\hline $6(-)$ c & BHPrE1 & $1 \times 10^{7}$ & $41.42 / 48.85$ & $1 \times 10^{6}$ & 30 minutes & $2.5 \mathrm{~mL}$ & FACS \\
\hline $6(-) d$ & PSMA* & $1 \times 10^{7}$ & $37.26 / 8.53$ & $\begin{array}{l}1 \mathrm{nmol} \\
\text { protein }\end{array}$ & 30 minutes & $2 \mathrm{~mL}$ & FACS \\
\hline$(+)$ & LNCaP & $1 \times 10^{7}$ & $25.67 / 15.63$ & $1 \times 10^{3}$ & 30 minutes & $2.5 \mathrm{~mL}$ & FACS \\
\hline
\end{tabular}

*PSMA is prostate-specific membrane antigen.

1X ab/am (Kaighn et al. 1979). The HPV-18-immortalized normal prostatic epithelium cell line RWPE-1 was obtained from ATCC and cultured in Defined KSFM (Gibco) plus 1X ab/am 
(Bello et al. 1997). The spontaneously-immortalized early prostate stem cell line NHPrE1 was a generous gift from Dr. Simon Hayward (Vanderbilt University) and cultured in the same media as BHPrE1 (Jiang et al. 2009).

\section{A.2.2 scFv Library and Growth}

A human non-immune scFv library displayed on the surface of Sacchoromyces cerevisiae with $10^{9}$ diversity was a gift from Dr. Dane Wittrup (Massachusetts Institute of Technology; Cambridge, MA) (Boder and Wittrup 1997; Feldhaus et al. 2003). The library had a full diversity of at least $10^{9}$ antibody fragments and has the ability to be serially propagated without loss of diversity (Feldhaus et al. 2003). The full library was cloned into the pPNL6 plasmid in the EBY100 yeast strain. It was originally amplified in standard dextrose media with casamino acids (SD+CAA), which contains $0.5 \%$ casamino acids, $2 \%$ dextrose, $0.17 \%$ yeast nitrogen base without ammonium sulfate amino acids, 0.53\% ammonium sulfate, 1.019\% sodium dihydrogen phosphate, $0.856 \%$ sodium monophosphate, and supplemented with $10 \mathrm{U} / \mathrm{mL}$ penicillin, 10 $\mu \mathrm{g} / \mathrm{mL}$ streptomycin, and $80 \mu \mathrm{g} / \mathrm{mL}$ ampicillin that had been vacuum filtered through a $0.22 \mu \mathrm{m}$ filter. The library was amplified to an OD of approximately 1.5 at $30^{\circ} \mathrm{C}$ with shaking at 250 RPM. Surface expression of the library was achieved by expressing the scFv under control of a galactose promoter in standard galactose plus casamino acids media (SG+CAA), which substituted $2 \%$ galactose, $2 \%$ raffinose, and $0.1 \%$ dextrose for the dextrose in SD+CAA. An initial input of 0.5 OD was amplified to approximately 1.5 OD with shaking at 250 RPM at $20^{\circ} \mathrm{C}$. After each induction growth period, surface expression of the library was confirmed by flow cytometry analysis. The monoclonal anti-HA tag antibody clone 16B12 conjugated to either DyLight 488 (Columbia Biosciences; Columbia, MD) or AlexaFluor 488 (Invitrogen; Grand Island, NY) was used to stain scFv-expressing yeast in yeast wash buffer (YWB) 
consisting of phosphate-buffered saline (PBS), 0.5\% bovine serum albumin, and 2 mM EDTA. The samples were run on either a Cell Lab Quanta SC (Beckman Coulter; Brea, CA) or a FACSCalibur (BD Biosciences; San Jose, CA) flow cytometer equipped with a $488 \mathrm{~nm}$ argon laser and $525 \mathrm{~nm}$ emission filter. At least 5\% partial induction of the surface library was confirmed and deemed sufficient for selection incubations.

\section{A.2.3 In Vitro Selection}

Seven rounds of Decoy-SELEX were performed in order to obtain a scFv MRE specific for the LNCaP prostate cancer cell line. The first three rounds of selection were performed by panning and the last four by fluorescence-activated cell sorting (FACS). The first three rounds of panning-based selection were performed directly in a T75 cell culture flask. For Round 1(+) selection, LNCaP cells were grown to $80-90 \%$ confluence and the media was removed. The cells were gently washed with calcium- and magnesium-free phosphate-buffered saline (PBS), which was removed. The cells were then incubated with $10^{10}$ yeast to ensure total representation of the library diversity. The naïve yeast library was amplified as discussed previously and scFv surface expression was induced. After surface expression confirmation, the library was suspended in $15 \mathrm{~mL}$ YWB. The library was placed into the flask containing LNCaP cells and placed on a $37^{\circ} \mathrm{C}$ shaker at 25 RPM for three hours. After incubation, yeast not bound to LNCaP cells were removed, and the LNCaP cells were gently washed three times with $15 \mathrm{~mL} \mathrm{YWB}$. It was then noticed, however, that LNCaP cells were less than $25 \%$ confluent in the flask, likely due to EDTA metal chelation and subsequent cell removal.

To determine optimal buffer conditions, separate 90\% confluent LNCaP T75 flasks were incubated with the following six buffers at $37^{\circ} \mathrm{C}$ with shaking at $25 \mathrm{RPM}$ for three hours: 1) YWB, 2) YWB with no EDTA, 3) YWB with no EDTA and 0.1\% FBS, 4) YWB with no EDTA 
and $0.5 \%$ FBS, 5)YWB with no EDTA and 1\% FBS, and 6) YWB with no EDTA and 10\% FBS. After incubation, remaining attached cells were visually estimated and compared to before incubation. Buffer 1 caused less than $25 \%$ of LNCaP to be attached, while Buffers 2-4 resulted in approximately 50\% attachment, and Buffers 5 and 6 caused almost no detachment (90\% confluent LNCaP cells), likely due to the FBS supplement. Therefore, Buffer 5 was chosen as an appropriate incubation buffer and named yeast selection buffer (YSB).

After determination of an appropriate incubation buffer, the Round $1(+)$ incubation was performed as described above, however with expressing yeast suspended in YSB. After three washes, $100 \mathrm{~mL}$ SD+CAA was added to the flask to allow for amplification of yeast bound to LNCaP cells. This was grown overnight, and this enriched library was prepared for a round of negative selection. For Round 1(-) selection, scFv-expressing yeast were suspended in YSB and incubated with rinsed HGPIN cells at $80-90 \%$ confluence for 30 minutes at $37^{\circ} \mathrm{C}$ with shaking at 25 RPM. The supernatant containing yeast-displayed scFvs not bound to HGPIN cells was removed and added to rinsed BPH-1 cells under the same conditions, removed again and added to rinsed BHPrE1 cells under the same conditions. The serially-incubated supernatant was centrifuged to obtain yeast, which were suspended in SD+CAA for amplification. Two more rounds of panning-based selection were performed in this manner, with decreasing incubation times for positive rounds and increasing incubation times for negative rounds.

The ensuing four rounds of selection were performed using FACS-based sorting. For Round 4(+) selection, LNCaP cells grown to 80-90\% confluence were fluorescently dyed with CFSE (Invitrogen) according to manufacturer's instructions. They were then dissociated from the flask with CellStripper reagent (Cellgro) to prevent cell surface protein digestion associated with trypsinization. Cells were then suspended in YSB and counted with a Scepter equipped 
with $60 \mu \mathrm{m}$ sensors (Millipore; Billerica, MA). In Round 4, all yeast were fluorescently dyed with Syto61 (Invitrogen) according to manufacturer's instructions. A total of $10^{7}$ yeast were suspended in YSB and mixed with $10^{6} \mathrm{LNCaP}$ in a volume of approximately $2.5 \mathrm{~mL}$. They were mixed by inversion for 30 minutes at $37^{\circ} \mathrm{C}$ and placed on ice before FACS sorting. The sample was then sorted with a FACSAria (BD Biosciences), with excitation at $488 \mathrm{~nm}$ from a sapphire solid state laser and $633 \mathrm{~nm}$ from a HeNe laser and $525 \mathrm{~nm}$ and $650 \mathrm{~nm}$ emission filters. Events identified as bound yeast and LNCaP cells were collected. In Round 4(+), there were two separate populations of bound cells which were collected and amplified separately. These were called the 'Hi' and 'Lo' populations and kept separate through the following rounds of selection and subjected to the same growth and selection conditions.

In Round 4(-)a, for each of the Hi and Lo populations, yeast were prepared for selection in the same manner and incubated with $10^{6}$ HGPIN cells dyed the same with inversion for 30 minutes at $37^{\circ} \mathrm{C}$ in YSB. The sample was subjected to FACS and events that indicated yeast that were not bound to HGPIN cells were collected and amplified. Those were then prepared for selection and incubated with $10^{6}$ dyed BPH-1 cells for Round 4(-)b and subjected to FACS with unbound yeast collected and amplified. The enriched library was then prepared for Round 4(-)c and subjected to FACS after incubation with $10^{6}$ dyed BHPrE1 cells. Unbound yeast were collected and amplified, then prepared for selection and incubated with $1 \mathrm{nmol}$ recombinant fulllength PSMA protein, which is expressed in non-prostatic normal and tumor tissue, as well as normal prostate cells (Silver et al. 1997) (Abnova; Walnut, CA), pre-incubated with AlexaFluor 488-conjugated anti-GST tag antibody (Invitrogen). The yeast which showed single fluorescence corresponding to Syto61 were kept and amplified. 
After post-Round 4 amplification, yeast from the Hi and Lo populations were prepared for Round $5(+)$ selection. They underwent the same process as before, however they were fluorescently labeled with anti-HA monoclonal antibody 16B12 conjugated to AlexaFluor 647 (Invitrogen). This was done for the following rounds to ensure only strongly-expressing yeast were collected. For Round $5(+)$ selection, $10^{7}$ yeast were incubated with $10^{6} \mathrm{LNCaP}$ cells. The yeast bound to LNCaP cells were collected, amplified, and prepared for Round 6(+) selection. A total of $10^{5}$ dyed LNCaP cells were used to increase stringency. Round 6(-) was performed similar to Round 4(-) with the exception of antibody-labeled yeast to increase stringency. Unbound yeast were collected, amplified, and prepared for Round 7(+). In that round, $10^{3}$ yeast were used to increase stringency and select the scFvs with highest affinity.

\section{A.2.4 Fluorescent Imaging of scFv Library Enrichment}

To visualize enrichment of the scFv library after 7 rounds of selection, fluorescent micrographs were obtained. LNCaP cells were grown in a 6-well culture dish with number 1.5 microscopic cover glasses to $90 \%$ confluency and fluorescently labeled with CFSE as described above. Yeast from the Round 0, post-Round 7(+) Lo, and post-Round 7(+) Hi libraries were cultured as described above. Surface expression of the scFv was confirmed by antibody labeling and flow cytometry as described. $10^{7}$ yeast were then labeled with anti-HA antibody conjugated to AlexaFluor 647 (Invitrogen) and incubated with LNCaP cells in $1 \mathrm{~mL}$ YSB in the 6-well dish. Cells were incubated at $37^{\circ} \mathrm{C}$ for 30 minutes with shaking at $25 \mathrm{RPM}$. The supernatant was removed and the cells were washed three times with $2 \mathrm{~mL}$ YSB. The cells were then fixed with 10\% formalin (Azer Scientific; Morgantown, PA) for 5 minutes and washed three times with 1 $\mathrm{mL}$ PBS then three times with $1 \mathrm{~mL}$ double-distilled water. Cover slips were dried and flipped onto a microscope slide prepared with 20 uL Fluorogel (Electron Microscopy Sciences; Hatfield, 
PA), and dried. Mounted cells were imaged on a Zeiss LSM 510 Confocal Microscope (Zeiss; Thornwood, NY) using an Argon laser at $488 \mathrm{~nm}$ and a HeNe laser at $688 \mathrm{~nm}$ for excitation with emission filters at 505-530 $\mathrm{nm}$ band pass and $650 \mathrm{~nm}$. Images were processed with Zeiss LSM Image Browser (Zeiss).

\section{A.2.5 Sequencing of scFv Library}

A representative sample of scFv-genes from the naïve library (Round 0 ) was sequenced to determine diversity of the library. Additionally, this was completed for the enriched postRound 2(-) and post-Round 3(-) libraries. It was also performed for each of the Hi and Lo populations following Round 4(-), Round 6(-) and Round 7(+) selections. To do this, yeast amplified from the previous round of selection were plated onto $2 \%$ agar plates containing $1 \%$ yeast extract, $2 \%$ peptone, and $2 \%$ dextrose (YPD). Yeast were grown for $36-48$ hours at $30^{\circ} \mathrm{C}$, and individual colonies were chosen for polymerase chain reaction (PCR) amplification.

Yeast colonies were picked and placed into double distilled water and boiled, which served as template for the PCR reaction. The reaction ingredients were as follows: $400 \mathrm{nM}$ forward (5'-GTACGAGCTAAAAGTACAGTG-3') and reverse (5'TAGATACCCATACGACGTTC-3’) pPNL6 primers (Eurofins MWG Operon), $250 \mu \mathrm{M}$ deoxynucleotide triphosphates, 5\% dimethyl sulfoxide, 1X Phusion Reaction Buffer (New England Biolabs, Ipswich, MA), 2 units Phusion High-Fidelity DNA polymerase (New England Biolabs), and double distilled water to $100 \mu \mathrm{L}$. Reactions conditions were: initial denaturation at $98^{\circ} \mathrm{C}$ for 30 seconds; 35 cycles of $98^{\circ} \mathrm{C}$ for 10 seconds, $50^{\circ} \mathrm{C}$ for 30 seconds, and $72^{\circ} \mathrm{C}$ for 30 seconds; and final extension at $72^{\circ} \mathrm{C}$ for 10 minutes. Results were analyzed using agarose gel electrophoresis and those PCRs that contained bands corresponding to the scFv gene were prepared for sequencing. They were purified using a PCR purification kit (IBI Scientific; Peosta, 
IA) and sent for DNA sequencing (Eurofins MWG Operon; Huntsville, AL) using both the forward and reverse pPNL6 primers. In total, at least 30 sequences were obtained for each enriched library noted above. Analysis was then performed by translating the DNA sequence to protein using the ExPASy translate tool (Swiss Institute of Bioinformatics; http://web.expasy.org/translate/). Hemagluttanin, c-Myc, and linker protein tag sequences, landmarks of the scFv expression scaffold, were identified to ensure sequence quality (Boder and Wittrup 1997). Sequences were compared for similarities and duplicates within an enriched library were identified to determine diversity of the library.

\section{A.2.6 Secretion and Purification of Selected scFvs}

From the Rounds 6(-) and 7(+) sequences, three scFvs were chosen for further study due to their abundance in the enriched libraries: HiR6.8, HiR7.6, and HiR7.8. Sequences were PCR amplified with the same ingredients and under the same conditions as above; however pPNL9 primers were used. For full length scFv sequences (HiR6.8), the forward pPNL9 (5'GACGTTCCAGACTACGCTGGTGGTGGTGGTTCTGCTA-3') and reverse pPNL9 (5’GGGTTAGGGATAGGCTTACCCTGTTGTTCTAGAATTCCG-3’) were used. For truncated scFv sequences (HiR7.6 and HiR7.8) containing only the heavy chain, forward pPNL9 and modified reverse pPNL9 (5'GGGTTAGGGATAGGCTTACCGAACTCTGAAGAGACGGTGACC-3’) were used. The PCR product was analyzed by agarose gel electrophoresis and the desired scFv-encoding gene was excised from the gel. DNA was purified from the gel using the GFX gel purification kit (GE Healthcare; Piscataway, NJ). Purified scFv-encoding DNA was then used for transformation of scFv-secreting yeast. 
To express and secrete the scFv, the encoding gene was cloned into the pPNL9 plasmid which was transformed into the YVH10 strain yeast, both generous gifts from Dr. Dane Wittrup (Massachusetts Institute of Technology). Prior to cloning, the pPNL9 plasmid was double digested with the restriction enzymes NotI and SfiI (New England Biolabs) and analyzed by agarose gel electrophoresis. The plasmid was prepared for further studies by phenol/chloroform extraction and ethanol precipitation. Transformation was performed by gap repair cloning and the LiAc TRAFO method as previously described (Gietz and Woods 2002). Briefly, YVH10 yeast with no plasmid were grown in YPD broth. A fresh sample was taken and centrifuged, then resuspended in the following: $67 \%$ of 50\% polyethylene glycol 3350, $0.1 \mathrm{M}$ lithium acetate, 13.9\% of $2 \mathrm{mg} / \mathrm{mL}$ boiled carrier DNA (salmon testes genomic DNA; Sigma), approximately 1 $\mu \mathrm{g}$ purified pPNL9-amplified scFv DNA, and approximately $0.3 \mu \mathrm{g}$ double-digested pPNL9 plasmid, with water to $360 \mu \mathrm{L}$. The yeast were incubated at $42^{\circ} \mathrm{C}$ for 1.5 hours, centrifuged, and suspended in water. They were then plated on selective SD+CAA agar with $0.8 \%$ tryptophan (Trp) and grown at $30^{\circ} \mathrm{C}$ for $48-96$ hours until individual colonies formed.

Individual colonies were chosen and subjected to colony PCR amplification as previously described, with the appropriate pPNL9 primer set used. The purified PCR product was sent for DNA sequencing to confirm the insert. The colony was then amplified in selective SD+CAA+Trp media overnight at $30^{\circ} \mathrm{C}$ with shaking at $250 \mathrm{RPM}$. Approximately $5 \times 10^{8}$ yeast were then centrifuged and suspended in $50 \mathrm{~mL}$ expression media containing $1 \%$ yeast extract, $2 \%$ peptone, $2 \%$ galactose, $2 \%$ raffinose, and $0.1 \%$ dextrose, plus antibiotics as listed above (YPG/R). Yeast were grown for approximately 72 hours with shaking at $250 \mathrm{RPM}$ at $20^{\circ} \mathrm{C}$ for secretion of scFv. 
After scFv secretion, supernatant was removed from the yeast by centrifugation. The supernatant was concentrated with a $9 \mathrm{kDa}$ centrifugal filter (Pierce). The concentrated scFv solution was then brought to a volume of $10 \mathrm{~mL}$ with PBS, $0.5 \mathrm{M} \mathrm{NaCl}$, and $0.05 \%$ Tween 20 . Then, $200 \mu \mathrm{L}$ Ni-NTA agarose resin (Qiagen; Valencia, CA) was added and mixed with inversion at $4^{\circ} \mathrm{C}$ for 2 hours for purification of 6-His tagged scFvs. The mixture was centrifuged and supernatant removed and washed three times with $10 \mathrm{~mL}$ PBS, $0.5 \mathrm{M} \mathrm{NaCl}$, and $0.05 \%$ Tween 20 solution. The scFv was then eluted from the agarose resin three times with $250 \mu \mathrm{L}$ of 200 mM imidazole in PBS. Pre-incubation, wash, and elution fractions were then concentrated using a $3 \mathrm{kDa}$ molecular weight centrifugal filter (Pierce).

Concentrated fractions were then analyzed using sodium dodecyl sulfate polyacrylamide gel electrophoresis (SDS-PAGE). Samples were mixed with loading dye, denatured and loaded into a 4-20\% Tris-HEPES gel. Samples were run and the gel was stained with Coomassie blue staining buffer. The gel was imaged, in which proper secretion and purification was confirmed by obtaining bands of the appropriate size for each scFv. Remaining elution fractions were combined, with extra imidazole removed with $3 \mathrm{kDa}$ centrifugal columns. The concentration of the scFv was determined using spectroscopy with a NanoDrop (Thermo Scientific; West Palm Beach, FL). The scFv was then diluted to a working concentration of $10 \mu \mathrm{M}$ in PBS.

\section{A.2.7 Affinity Binding Assays with scFvs}

In order to determine the binding affinity of each secreted scFv with the LNCaP cell line, saturation binding assays were performed with flow cytometry with few modifications to previous work (Benedict et al. 1997; Boder et al. 2000). LNCaP cells were grown to 80-90\% confluency and removed from the flask with CellStripper reagent. The cells were counted with a Scepter equipped with $60 \mu \mathrm{m}$ sensors. Then, 2 X $10^{5}$ cells were placed into a $500 \mu \mathrm{L}$ total 
volume of YSB. LNCaP cells incubated with no antibody served as a negative fluorescent control, and LNCaP cells incubated with $1 \mu \mathrm{L}$ anti-PSMA antibody conjugated to AlexaFluor 488 (BioLegend; San Diego, CA) served as a positive fluorescent control. For saturation binding assays, the appropriate amount of each scFv was incubated on ice for 30 minutes with $2 \mu \mathrm{L}$ of the monoclonal anti-HA antibody clone 16B12 conjugated to DyLight 488 (Columbia Biosciences). Concentrations of 0, 1, 10, 100, 1000, 10000, 50000, 100000, 150000, 200000 pM of the scFv were used. The pre-incubated scFv and secondary antibody were mixed with LNCaP cells and placed on a rotisserie at $37^{\circ} \mathrm{C}$ for 30 minutes. After this period, the cells were centrifuged for 7 minutes at $300 \mathrm{xg}$ at $4^{\circ} \mathrm{C}$. The supernatant was removed and the cells were resuspended in $500 \mu \mathrm{L}$ YSB.

Samples were run on a FACSCalibur (BD Biosciences) as previously described. The mean fluorescence intensity (MFI) for each sample was recorded. The MFI for the $0 \mathrm{pM}$ incubation, which had only the secondary fluorescent antibody, served as background fluorescence and was subtracted from the MFI of each incubation. These data were graphed using Origin 8 software (OriginLab Corporation; Northampton, MA). The data were fit with a nonlinear regression model of single-event binding given by the equation, $\mathrm{Y}=\left(\left(\mathrm{B}_{\max } * \mathrm{X}\right) /\left(\mathrm{K}_{\mathrm{d}}+\right.\right.$ $\mathrm{X}))+N S^{*} \mathrm{X}$, where $\mathrm{B}_{\max }$ is maximum binding, $\mathrm{K}_{\mathrm{d}}$ is the dissociation constant, and NS is nonspecific binding. Binding assays for HiR6.8 and HiR7.8 were performed in triplicate, with average dissociation constants calculated and standard errors derived from the equation. Binding assays for HiR7.6 will be performed in the future in triplicate.

\section{A.2.8 Specificity Binding Assays of scFvs}

In order to determine the specificity of MRE binding, cross-binding assays were performed with the cell lines used as negative targets in the selection. LNCaP, HGPIN, BPH-1, 
and BHPrE1 cells were grown to 80-90\% confluency and removed from the flask with CellStripper reagent. Cells were then counted with a Scepter using $60 \mu \mathrm{m}$ sensors and 2 X $10^{5}$ cells were placed into a final volume of $500 \mu \mathrm{L}$ YSB. For each cell line, cells with no antibody served as a negative control. Concentrations of 0 or 100000 pM scFv were incubated with the secondary anti-HA AlexaFluor 488 antibody for 30 minutes on ice. Each of these were then mixed with each cell line and incubated on a rotisserie at $37^{\circ} \mathrm{C}$ for 30 minutes. The cells were then centrifuged, the supernatant removed, and they were resuspended in $500 \mu \mathrm{L}$ YSB. Each sample was then run on a FACSCalibur as previously described and the MFI recorded. The MFI of the $0 \mathrm{pM}$ incubation, which had only the secondary fluorescent antibody, served as background fluorescent labeling and was subtracted from the 100000 pM incubation. Data were normalized to LNCaP binding, which was set to 100\%, and graphed to show comparative binding between the cell lines. HiR6.8 and HiR7.8 assays have been performed in triplicate, with average values calculated and Student's T-tests performed to determine statistical significance. HiR7.6 will be assayed in the future in triplicate.

In order to determine binding to other prostatic cell lines used not used in the selection, cross-binding assays were performed. LNCaP, the androgen insensitive prostate cancer cell lines DU-145 and PC-3, and the normal prostatic cell lines RWPE-1 and NHPrE1 were used for this experiment. The cells were prepared as noted for the other cross-binding assays and incubated with the scFv as described above. Samples were run on a FACSCalibur and the MFI was normalized and graphed as noted. HiR6.8 and HiR7.8 have been performed in triplicated, with average values calculated and Student's T-tests performed to determine statistical significance. HiR7.6 will be assayed in the future in triplicate. 


\section{A.2.9 Fluorescent Imaging of scFv Binding}

In order to visualize binding of each scFv, fluorescent images were taken. LNCaP cells were grown in a 6-well culture dish with number 1.5 microscopic cover glasses to $~ 90 \%$ confluency. For each scFv, a $100 \mathrm{nM}$ concentration was pre-incubated with anti-HA antibody conjugated to DyLight 488 as described for binding assays, in addition to LNCaP cells with antiPSMA antibody, and those with secondary antibody only in a total volume of $500 \mu \mathrm{L}$ YSB. Cells were incubated at $37^{\circ} \mathrm{C}$ for 30 minutes with shaking at $25 \mathrm{RPM}$. The supernatant was removed and the cells were washed once with $2 \mathrm{~mL}$ YSB. The cells were then fixed with 10\% formalin (Azer Scientific; Morgantown, PA) for 5 minutes and washed three times with $1 \mathrm{~mL}$ PBS then three times with $1 \mathrm{~mL}$ double-distilled water. Cover slips were dried and flipped onto a microscope slide prepared with 20 uL Fluorogel (Electron Microscopy Sciences; Hatfield, PA), and dried. Mounted cells were imaged on a Zeiss AxioImager Z2 Fluorescent Microscope (Zeiss; Thornwood, NY) using an Argon laser at $488 \mathrm{~nm}$ for excitation with a band pass emission filter at 505-530 nm.

\section{A.3 Results and Discussion}

\section{A.3.1 Selection of MREs}

In order to obtain prostate cancer cell-specific MREs, seven rounds of the Decoy-SELEX

process were completed. Sequencing of the scFv-encoding plasmid in the initial naïve library, as well as after Round 2 and Round 3 of panning selection, was performed. Out of at least 30 sequences obtained, there were no duplicate sequences in any library. Additionally, there were no duplicates between the libraries. This suggests that all three libraries were highly diverse, with no particular sequence having been selected for. 
After three rounds, FACS-based selections were performed. In Round $4(+)$, there were two separate populations of yeast binding to LNCaP cells. One was characterized by having relatively high Syto61 (yeast) fluorescence with low CFSE (LNCaP) fluorescence, and this was designated the 'Hi' population. The other was characterized by having relatively low Syto61 (yeast) fluorescence with high CFSE (LNCaP) fluorescence, and was designed the 'Lo' population. These were sorted separately and kept separate through the remaining rounds of selection, while each was subjected to identical growth and selection conditions.

After the negative selections in Round 4, sequencing of the scFv-encoding genes in the library revealed duplicate sequences within individual libraries. The Lo population was comprised of $29 \%$ HiR7.8 and between $3 \%$ and $13 \%$ of 13 other molecules (Figure A.2).

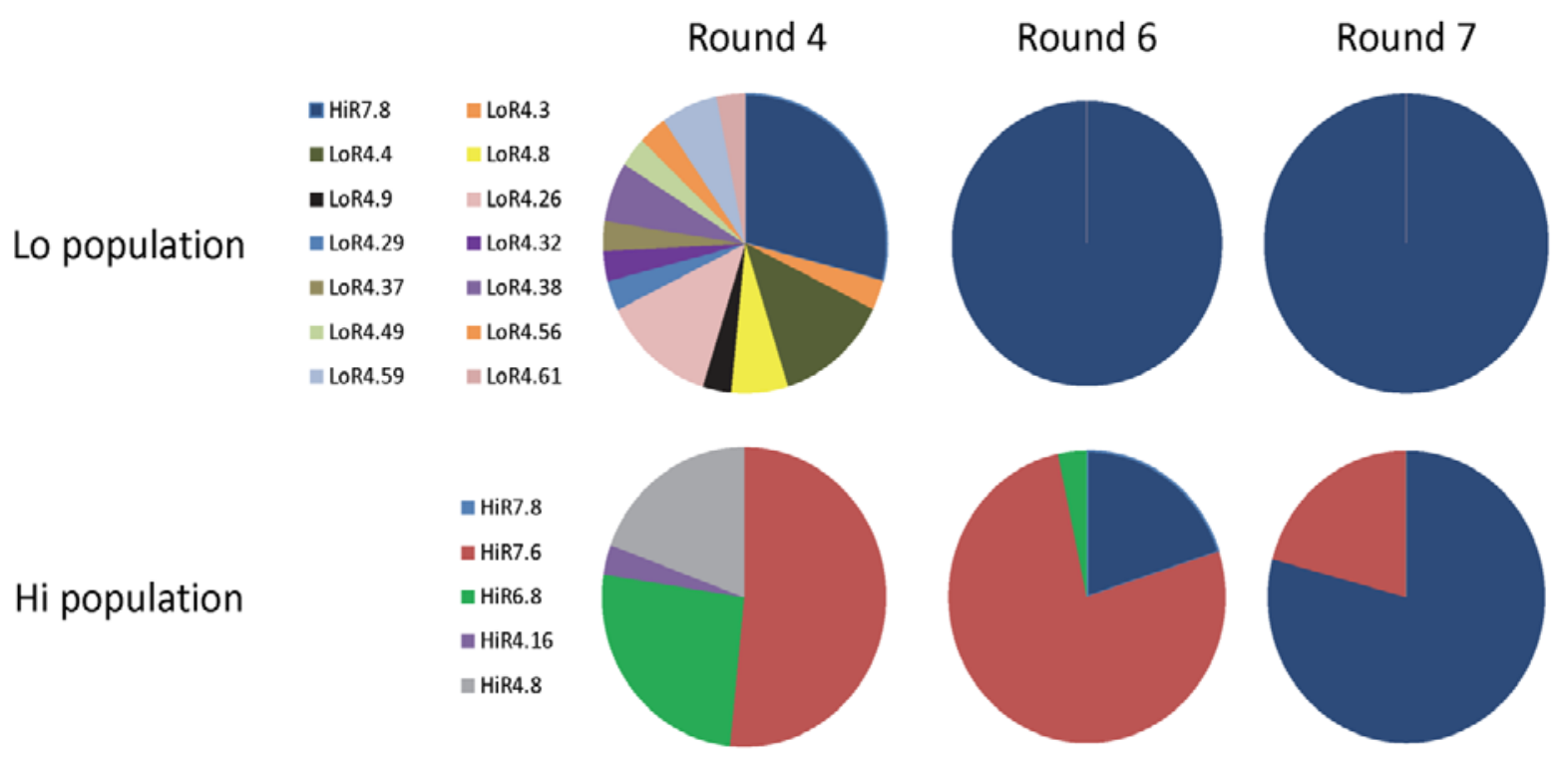

Figure A.2. Enrichment of the Lo and Hi yeast-displayed scFv populations after FACSbased selection rounds. Evolution of library diversity after Round 4, the first round of FACSbased selection, Round 6, and Round 7. Lo population is the top row the Hi population on the bottom. The names of the sequences represented by each color in the pie charts are shown to the left of Round 4. 
Interestingly, HiR7.6 and HiR6.8 were not present in the Lo population. In the Hi population, however, HiR7.6 comprised 52\% of the population, while HiR6.8 was $26 \%$. One other scFv was $3 \%$ and a final molecule was 19\%. Interestingly, HiR7.8 did not appear in the Round $4 \mathrm{Hi}$ population. It is also of note that HiR7.6 and HiR7.8 did not appear in any previous library sequencing, however HiR6.8 did appear once in the Round 3 library.

Following the sixth round of selection, the Lo and Hi libraries were sequenced. In the Lo population, 30 out of $30(100 \%)$ of the sequenced molecules were HiR7.8, and there were no longer any other detectable scFvs in the library (Figure A.2). This suggests that, within this population, HiR7.8 was strongly enriched for in Rounds 5 and 6 of selection. In the Hi population, $77 \%$ of the library was HiR7.6 while 3\% was HiR6.8. Interestingly, HiR7.8 now comprised 20\% of the library, and no other molecules were present. This suggests that HiR7.6
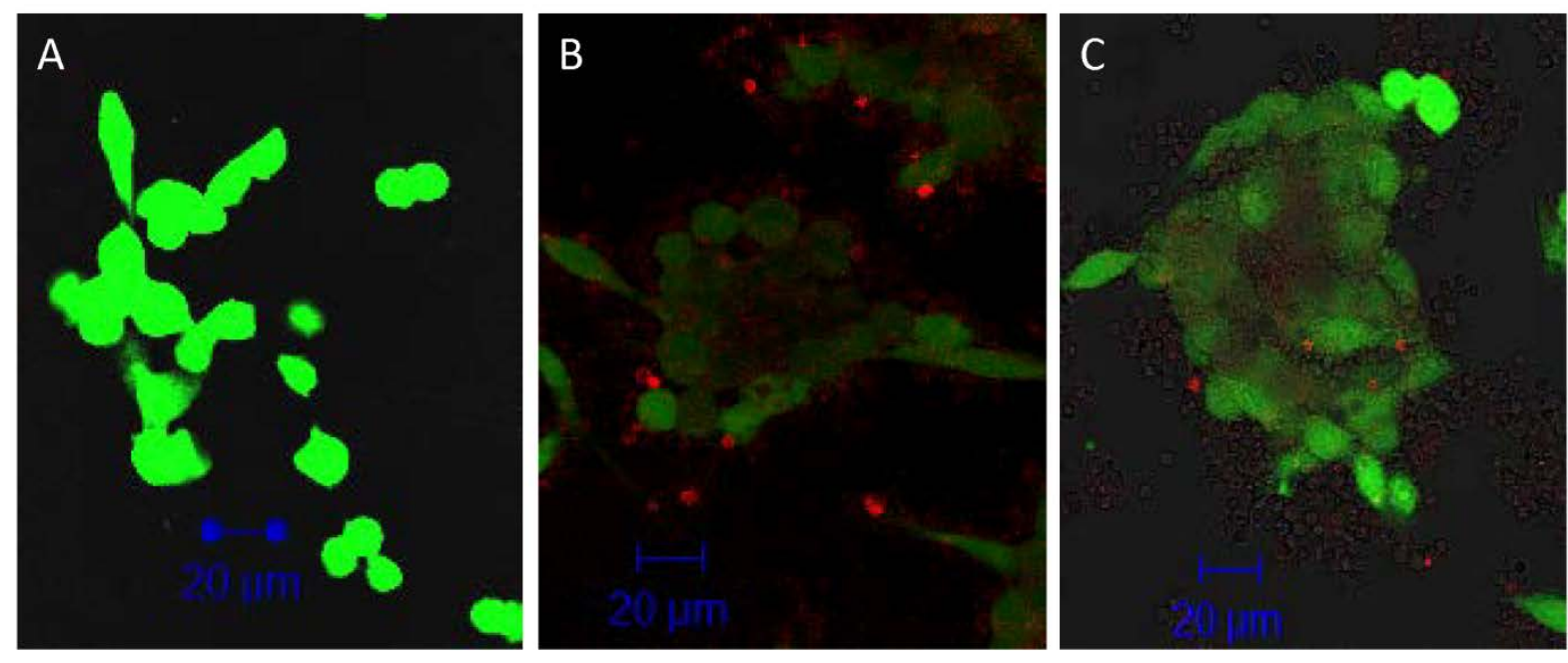

Figure A.3. Fluorescent micrographs of yeast binding enrichment. Confocal microscope images at 200X of LNCaP cells (green) incubated different scFv-displaying yeast libraries. (a) LNCaP incubated with Round 0 library. (b) LNCaP incubated with Round 7(+)Lo library. (c) LNCaP incubated with Round 7(+) Hi library. Images show enrichment of yeast library through seven rounds of selection for yeast-displayed scFv specific for LNCaP cells. 
outcompeted HiR6.8 within this population. It also suggests that HiR7.8 was present previously in the library, but not detected, and had outcompeted HiR6.8.

Following a very stringent Round $7(+)$ selection, using 10,000 fewer target cells than yeast, and 1,000 times fewer LNCaP that Round 6(+), sequencing further revealed the scFvs present in the library. In the Lo population, 100\% of the molecules present were again HiR7.8 (Figure A.2). While there may have been other scFvs in the library, it is unlikely after two consecutive rounds of not detecting any besides HiR7.8. In the Hi population, HiR6.8 was no longer detected, while HiR7.6 was now just $16 \%$ of the library. HiR7.8 comprised $84 \%$ of the library, suggesting it strongly outcompeted HiR7.6. It is possible that if more rounds of selection were completed under more stringent conditions, HiR7.8 would totally comprise the Hi population as well.

Visualization of library enrichment for scFvs binding to LNCaP cells was performed. This provided evidence that few or none of the molecules in the Round 0 library were binding to LNCaP cells (Figure A.3). However, after seven rounds of selection, both the Lo and Hi populations were strongly specific for LNCaP cells (Figure A.3). This, along with the sequence evolution, validates the sequence enrichment capabilities of the novel Decoy-SELEX process. From this obvious enrichment through seven rounds of Decoy-SELEX, HiR7.8 was chosen as the most likely prostate cancer cell-specific MRE and underwent further characterization. However, to determine why it outcompeted HiR6.8 and HiR7.6, those molecules were also chosen for further characterization. It is also important to note that HiR6.8 is a full-length scFv, comprising both a heavy and light chain, with a predicted molecular weight of approximately $30.6 \mathrm{kDa}$ for the scFv itself (Table A.2). HiR7.8 and HiR7.6, however, only contain a heavy chain, as they are truncated just before the chain linker. They have a predicted molecular weight 
of approximately 16.0 and $15.6 \mathrm{kDa}$, respectively, and have $81 \%$ sequence identity and $86 \%$ sequence similarity. Truncated sequences containing only heavy chains were found in sequencing of the naïve library in the original published description (Feldhaus et al. 2003). This may be relevant in vivo, as it has been shown that smaller scFvs have greater tumor penetration ability than larger antibodies (Colcher et al. 1998).

Table A.2. Amino acid sequence of selected prostate cancer cell MREs.

\begin{tabular}{|c|c|c|}
\hline $\begin{array}{l}\text { Sequence } \\
\text { Name }\end{array}$ & Sequence* & $\begin{array}{l}\text { Predicted } \\
\text { Molecular } \\
\text { Weight }\end{array}$ \\
\hline HiR6.8 & $\begin{array}{l}\text { DVPDYALQASGGGGSGGGGSGGGGSASQVQLVQSGAEVKKPGASVKVSCKASGY } \\
\text { TFTDYYMHWVRQAPGQGLEWMGWINPNSGGTNYAQKFQGRVTMTRDTSISTAYM } \\
\text { ELSSLRSDDTAIYYCARDADSGSMSAIYWYFNLWGRGTLVTVSSGILGSGGGGS } \\
\text { GGGGSGGGGSDIQLTQSPSSLSASVGDRVTITCRASQSISRFLNWYQQKPGKAP } \\
\text { KLLIYGGSSLQSGVPSRFSGGGSGTDFTLTISSLQPEDFATYYCQQSYSKFWTF } \\
\text { GQGTKVEIKSGILEQKLISEEDL }\end{array}$ & $30.6 \mathrm{kDa}$ \\
\hline HiR7.6 & $\begin{array}{l}\text { DVPDYALQASGGGGSGGGGSGGGGSASQVQLQESGPGLVKPSGTLSLTCAVSGG } \\
\text { SISSSNWWSWVRQPPGKELEWIGEIHHSGSTNYNPSLKSRVTISVDKSKNQFSL } \\
\text { KMRSVTAADTAVYYCARVEEWPYDALDMWGQGTMVTVSSEF }\end{array}$ & $15.6 \mathrm{kDa}$ \\
\hline HiR7.8 & $\begin{array}{l}\text { DVPDYALQASGGGGSGGGGSGGGGSASQVQLQESGPGLVKPSQTLSLTCTVSGD } \\
\text { SIYSSGHYWSWVRQHPGKGLEWIGYIYASGRTYYNPSLESRVTMSVDTSKNQSS } \\
\text { LKLTSVTAADTAVYYCARDDSRTWYKAFDTWGQGTMVTVSSEF }\end{array}$ & $16.0 \mathrm{kDa}$ \\
\hline
\end{tabular}

* Underlined amino acids represent the heavy chain. Double underlined amino acids represent the light chain. HiR7.6 and HiR7.8 do not contain a light chain. Non-underlined amino acids represent scFv structural elements.

\section{A.3.2 Affinity Binding Assays of scFvs}

Once HiR6.8, HiR7.6, and HiR7.8 were identified for further characterization, each was cloned into a secretion vector, expressed, and purified. HiR6.8 and HiR7.8 were used in fluorescent saturation binding assays with LNCaP cells as described above, plotted, and fit with nonlinear regression analysis (Figure A.4). HiR6.8 exhibited an equilibrium dissociation constant $\left(\mathrm{K}_{\mathrm{d}}\right)$ of $33.19+/-22.18 \mathrm{nM}$ (Table A.3). The binding affinity of HiR7.6 for LNCaP 
cells will be assayed in the future. The binding affinity of HiR7.8 is $27.28+/-15.85 \mathrm{nM}$. The slightly higher affinity of HiR7.8 for LNCaP cells may be a partial explanation as

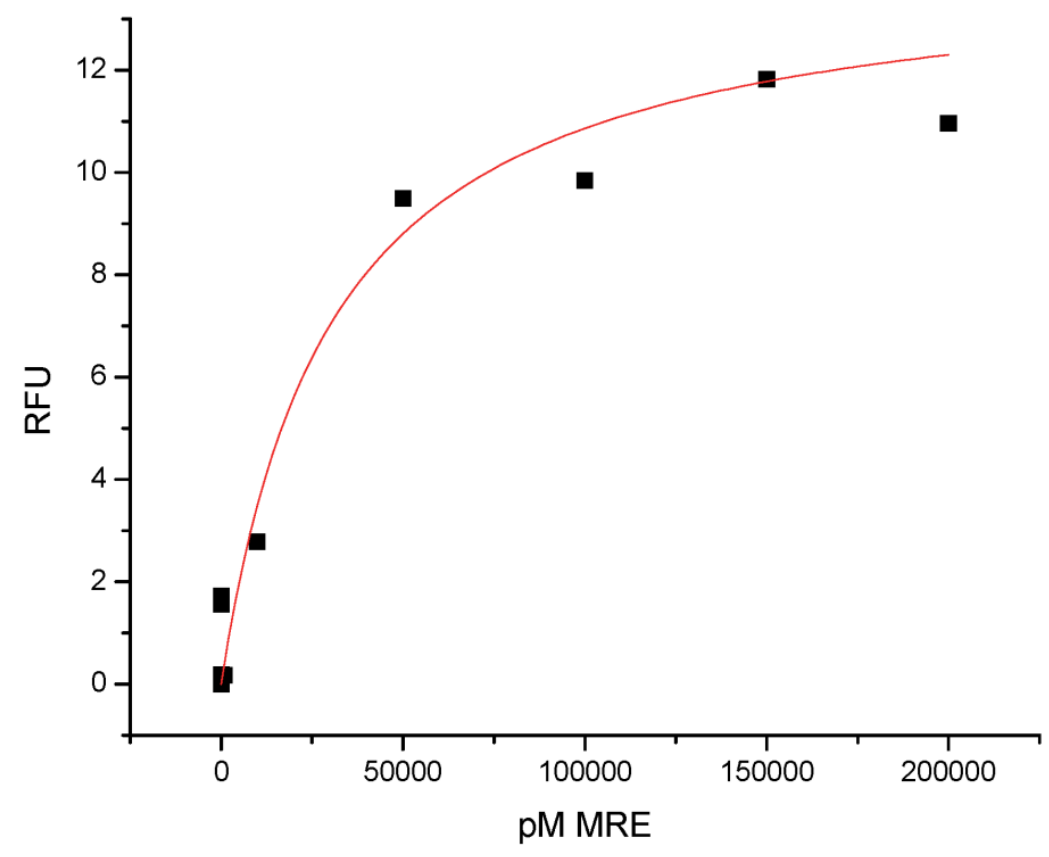

Figure A.4. Plot of fluorescent equilibrium binding assay of HiR6.8 and LNCaP cells. Calculated dissociation constant $\left(\mathrm{K}_{\mathrm{d}}\right)$ of this assay is $30.52+/-27.08 \mathrm{nM}$.

Table A.3. Equilibrium dissociation constant $\left(\mathrm{K}_{\mathbf{d}}\right)$ of prostate cancer cell MREs.

$\begin{array}{lll}\text { HiR6.8 } & \text { HiR7.6 } & \text { HiR7.8 } \\ 33.19+/-22.18 \mathrm{nM} & * & 27.28+/-15.85 \mathrm{nM}\end{array}$

Data are reported as $\mathrm{K}_{\mathrm{d}}+/$ - Standard Error as given by the binding equation. “*” denotes that the data are yet to be acquired.

to why it was enriched for throughout Decoy-SELEX. The dissociation constants for each of these scFvs is in the low-nanomolar range, which is similar to previous reports of scFvs isolated from the selection process (Schier et al. 1996; Benedict et al. 1997; Böldicke et al. 2001). More importantly, it is in a range that has previously been shown to confer strong pharmacokinetic properties (Adams et al. 1998; Jackson et al. 1998). It is possible that further mutagenesis and screening may select for scFvs with higher affinity for the cell surface target (Schier et al. 1996; Boder et al. 2000). 
Binding of both HiR6.8 and HiR7.8 to LNCaP cells is further evidenced by fluorescent micrographic analysis. Binding of each scFv is clear when pre-incubated with a secondary fluorescent antibody then with LNCaP cells and imaged for fluorescent staining (Figure A.5). Fluorescence is much brighter than LNCaP cells incubated with fluorescent secondary-only control. Interestingly, both antibodies have a semi-circular or horseshoe-like staining pattern for each cell. Therefore, fluorescence may be visualized in the cytosol surrounding the nucleus. It may be possible that the images represent cellular uptake of the scFv-secondary antibody complex. This has previously shown to be possible and even likely with scFvs alone or in complex with other molecules (Gao et al. 2003; Nielsen et al. 2006; He et al. 2010). This may have an important role in the clinical application of these MREs. The potential ability to use these scFvs as therapeutic targeting agents in the form of antibody-drug conjugates (ADCs) is enhanced by scFv internalization (Schrama et al. 2006). Potential uptake of the scFv by LNCaP cells may be explored in future studies. First, confirming cytoplasmic staining using a nuclear dye such as DAPI would be useful. This could then be furthered by subcellular fractionation experiments. These are potentially interesting further directions stemming from fluorescent imaging of scFv binding.

\section{A.3.3 Specificity Binding Assays of scFvs}

Purified HiR6.8 and HiR7.8 were each assayed for their ability to bind to cell lines other than LNCaP. HiR7.6 will be assayed in the future for its binding to other cell lines. These included the negative targets in the selection process, HGPIN, BPH-1, and BHPrE1 (Figure A.6 a and Figure A.7a). They also included androgen-independent prostate cancer cell lines DU-145 and PC-3 as well as normal prostate epithelium cell lines RWPE-1 and NHPrE1 (Figure A.6b and Figure A.7b). 


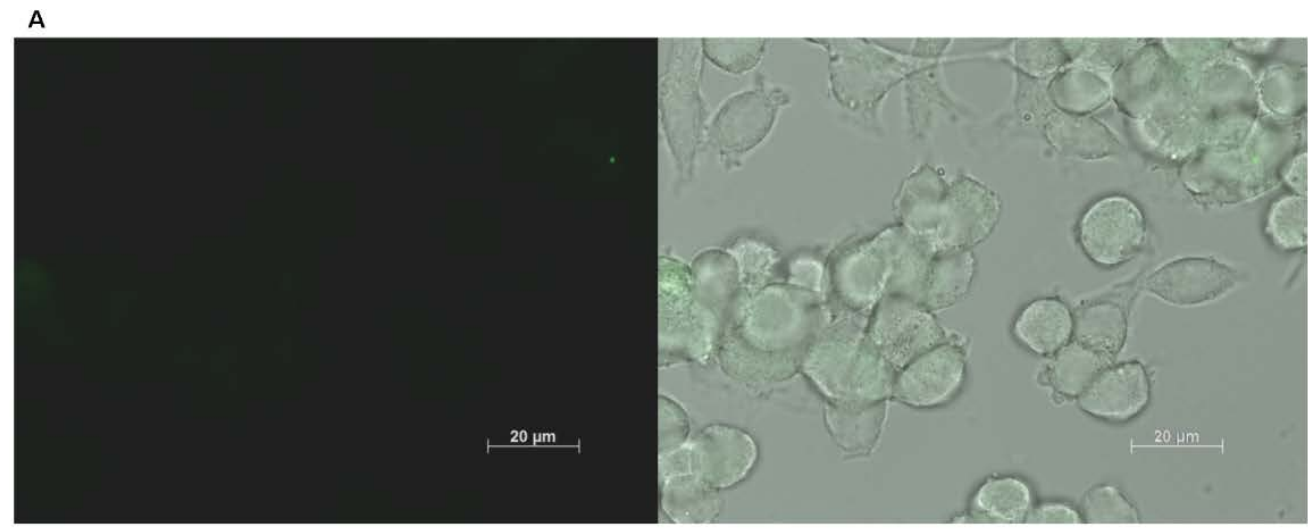

B

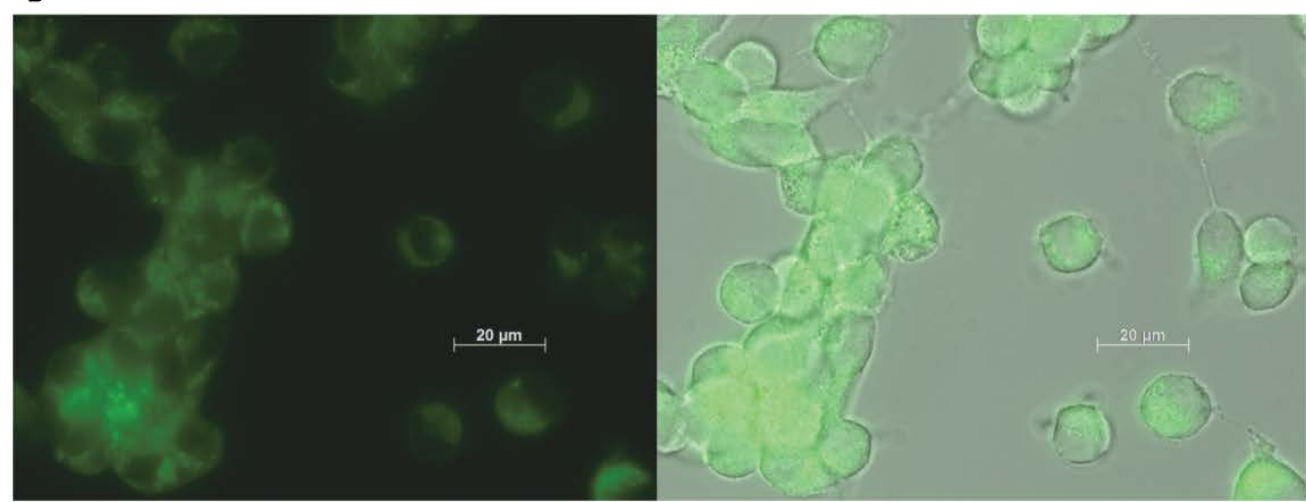

C

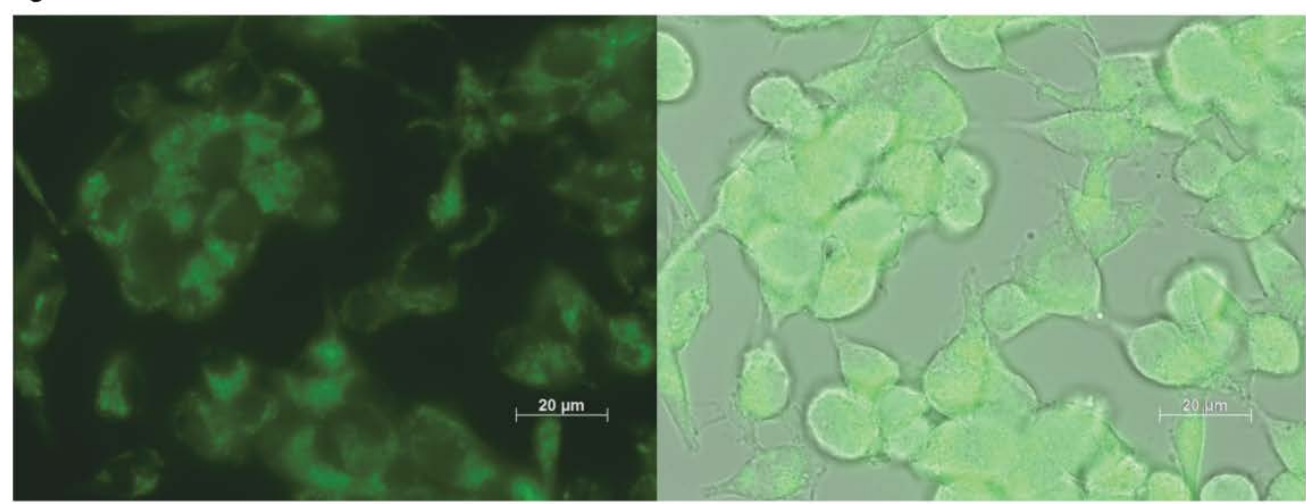

Figure A.5. Micrographs of scFv binding to LNCaP cells. Each panel shows micrographs at 630X of fluorescence from AlexaFluor 488 in green (left) and combined fluorescent plus brightfield image (right). (a) LNCaP cells incubated with only secondary antibody. (b) LNCaP cells incubated with HiR6.8 plus secondary fluorescent antibody. (c) LNCaP cells incubated with HiR7.8 plus secondary fluorescent antibody.

From these experiments, it is clear that HiR6.8 binds to all three negative cell lines as well as the other two androgen-dependent prostate cancer cell lines assayed. Binding to many of these lines was as good as or better than binding to LNCaP cells. This is a likely explanation as 
to why HiR6.8 was eventually eliminated from both populations, in spite of its low dissociation constant (Figure A.6). HiR7.8, however, showed excellent selectivity for LNCaP cells (Figure A.7). Binding to HGPIN, BPH-1, and BHPrE1 cells all was much less than to LNCaP, in fact they were negative. This means that the MFI of cells incubated with 100000 pM HiR7.8 and secondary fluorescent antibody was less fluorescent than cells incubated with secondary fluorescent antibody only. This can be explained by pre-incubation and binding of the scFv with the secondary antibody, causing background non-specific binding of the secondary antibody to the cells to be less. Therefore, the negative relative MFI suggests that the antibody is present and binding to the secondary antibody, but not to the cells. Additionally, binding to the four cell lines not used in the selection was either negative or much less relative to binding to LNCaP cells. In sum, this suggests that HiR7.8 is highly specific to LNCaP cells and therefore useful in both the therapeutic targeting and disease detection of prostate cancer.

Biologically, the selectivity of HiR7.8 for LNCaP cells is important. There is a complete absence of binding to all benign cell lines studied, whether they represent normal prostatic cells, $\mathrm{BPH}$, or PIN. This suggests that the cell surface molecule HiR7.8 is binding to is not displayed on the surface of benign prostatic cells. This is supported by previous studies that have identified many genes differentially expressed between the benign conditions represented here and prostate cancer cells (Chakrabarti et al. 2002; Zeng et al. 2004; Chen et al. 2011).

Additionally, there is no binding to the androgen-independent prostate cancer cell lines DU-145 or PC-3. This leads to the conclusion that the cell surface antigen is displayed in the earlier, androgen-dependent stage of prostate cancer. This is possible considering previous studies have found a wide array of genes that are differentially expressed from androgen 
A

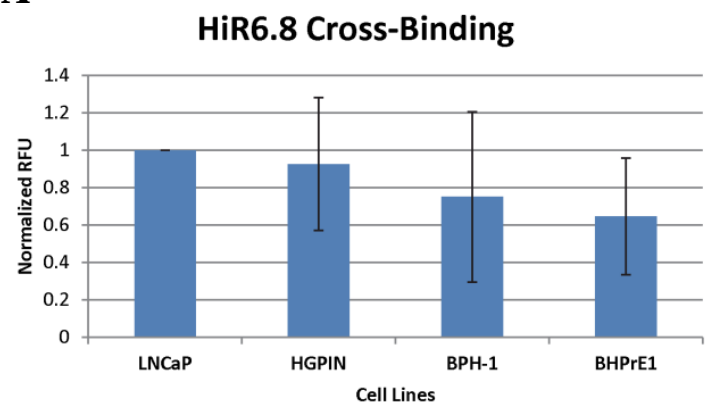

B

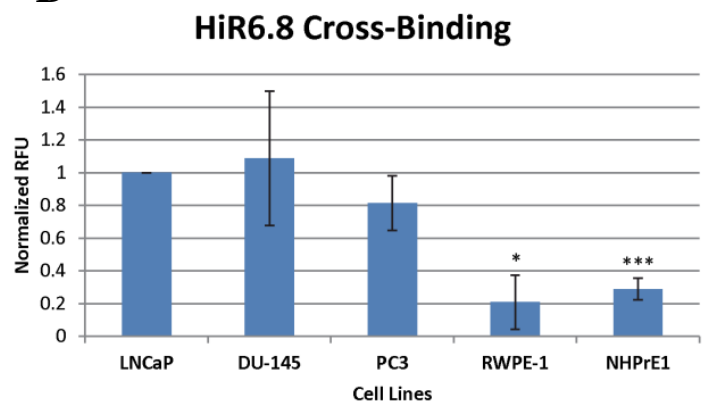

Figure A.6. Cross-binding of HiR6.8 to negative targets and other cell lines. Binding of HiR6.8 to (a) each cell line used in the selection or (b) or prostatic cell lines. Data are normalized to LNCaP binding at $100 \%$ for comparison. "*" represents $\mathrm{p}<0.05$, "***" represents $\mathrm{p}<0.001$.

A

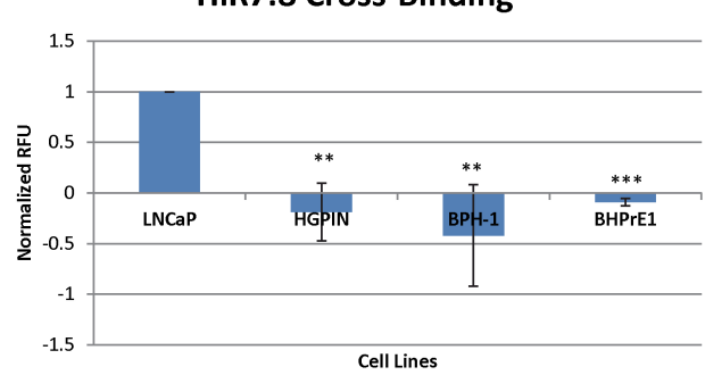

B HiR7.8 Cross-Binding

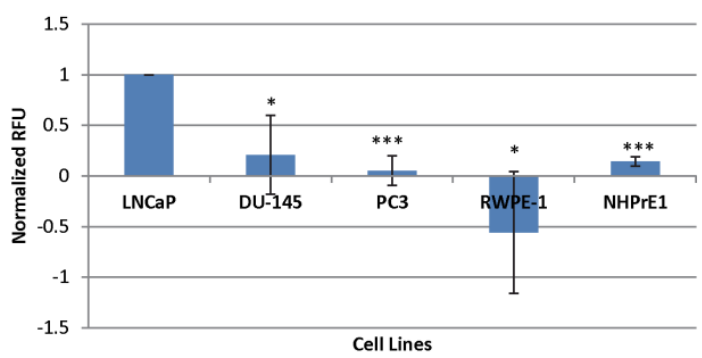

Figure A.7. Cross-Binding of HiR7.8 to negative targets and other cell lines. Binding of HiR7.8 to (a) each cell line used in the selection or (b) other prostatic cell lines. Data are normalized to LNCaP binding at $100 \%$ for comparison. "*" represents $\mathrm{p}<0.05$, "**" represents $\mathrm{p}<0.01, " * * * "$ represents $\mathrm{p}<0.001$.

dependent to independent disease progression, many of which are downregulated (Amler et al. 2000; Karan et al. 2002; Chen et al. 2006). In fact, downregulation of expression levels from LNCaP to both DU-145 and PC-3 cells has been found before (Yang et al. 1998; Aalinkeel et al. 2004; Okamura et al. 2008; Liu et al. 2009) It is unclear if HiR7.8 would bind to other androgen-dependent prostate cancer cells, or cells earlier in the progression of the disease, however future work may determine this. Determining binding will be aided by identifying the exact cell surface molecule to which HiR7.8 is binding, as has been done before with other MREs (Robert et al. 2006; Rérole et al. 2011). This type of work has even identified novel cell surface molecules previously unknown to be involved in disease progression (Berezovski et al. 
2008). It is therefore possible that this work not only created a novel targeting agent, but also identified a novel target.

HiR6.8, however, is not specific for any one type of cell. This may be because it is binding to some widely-expressed prostate-specific gene such as PSMA (Wright et al. 1995), or because it is binding to any one of a number of common cell surface molecules. Determining the cell surface antigen for HiR6.8 will be important as it may be useful as a broad marker of prostatic cells similar to PSCA or p63 (Signoretti et al. 2000; Tran et al. 2002), or it may be useful in marking a particular cell surface antigen such as previous scFvs for ICAM-1 (Conrad et al. 2009). Therefore, in spite of its spectrum of binding, the high affinity of HiR6.8 for its cell surface target will allow the scFv to be useful for future applications, even if it is not useful in the specific targeting of prostate cancer cells.

The high affinity and specificity of HiR7.8 for LNCaP prostate cancer cells suggest it has potential in future therapeutic and diagnostic applications in prostate cancer. It is unclear whether it will bind to prostate cancer tissue, however it is likely that it will based on binding specificity and affinity similar to previously-selected MREs (Ni et al. 2011). This will rely on surface expression of its cognate antigen. The selective scFv will likely be useful for drug delivery as part of an antibody-drug conjugate (Senter 2009), for chemotherapeutic delivery (Sievers and Linenberger 2001), for radiotherapy delivery (Regino et al. 2009), or possibly even as a therapeutic by itself, depending on the function of the cell surface molecule (Stebbing et al. 2000). Additionally, it will likely have use in specific diagnosis of prostate cancer, whether coupled with an in vivo imaging agent (Elsässer-Beile et al. 2009; Veiseh et al. 2010), a capture agent for circulating tumor cells (Nagrath et al. 2007), or as a tissue stain after biopsy (Johnston et al. 1986). Finally, identification of the cell surface markers which these MREs bind to may 
possibly identify novel proteins, new functions, or expression patterns that aid in treatment and diagnosis of prostate cancer.

\section{A.4 Conclusions}

This work has selected a MRE with high affinity for the androgen-dependent prostate cancer cell line LNCaP. Furthermore, the selected MRE has a high selectivity for the androgendependent prostate cancer cell line, and not for benign prostatic cell lines or for androgenindependent prostate cancer cells. These characteristics will allow the selected scFv to be useful for both therapeutic and diagnostic applications in the clinical treatment of prostate cancer. With targeted therapeutics and more specific diagnostics, it is possible that the disease burden of the most-diagnosed cancer among males in the United States will be lessened. 


\section{Appendix B}

\section{In Vitro Selection of Single-Stranded DNA Molecular Recognition Elements for the Pesticide Malathion}




\section{B.1 Introduction}

Malathion is the mostly commonly-used organophosphate pesticide in the United States. Approximately 16.7 million pounds are used annually, with 12 million pounds being applied to crops (Wilson et al. 2003). Many other uses include in sewage systems, plant nurseries, home use, and recreational land maintenance (Wilson et al. 2003). Three major historical applications in the U.S. were Boll Weevil eradication, Medfly suppression, and mosquito suppression (Newhart 2006). Its widespread use portends the benefits of malathion usage.

Due to its many uses, malathion has become an environmental contaminant with multiple routes of exposure. Aerial applications often drift to non-target locations, including waterways, up to 200 meters away (LeNoir et al. 1999; Newhart 2006). This method can also lead to volatilization of up to $22.6 \%$ of that applied, leading to reduced air quality levels and vaporized contamination spread potentially over large distances (Turner et al. 1991; LeNoir et al. 1999; Ferrari et al. 2003). After one large application, malathion was found in freshwater ponds at levels up to $49.4 \mu \mathrm{g} / \mathrm{L}$ (Segawa et al. 1991). Additionally, runoff due to storms after application has led to levels up to $787.1 \mu \mathrm{g} / \mathrm{L}$ being found (Ando et al. 1996). Detection in groundwater and surface water has also occurred at levels up to $6 \mu \mathrm{g} / \mathrm{L}$ during normal conditions (Newhart 2006). In the state of West Virginia, malathion has been found at levels that exceed guidelines for environmental contamination (Ferrari and Assessment 1997; Ator 1998). Furthermore, one study found that $33.7 \%$ of corn grain, $27.8 \%$ of cultivated and frozen blueberries, $24.6 \%$ of strawberries, and $19.3 \%$ of celery samples on the market tested had detectable levels of malathion (USDA 2009). Clearly, release of malathion into the environment and its interaction with humans and other organisms is occurring. 
The presence of malathion in the environment is of particular concern to human health. The U.S. Environmental Protection Agency categorizes the pesticide as having "suggestive evidence of carcinogenic potential" (USEPA 2012). This is supported by studies showing induction of mammary epithelial cell proliferation and other carcinogenic effects by malathion through acetylcholinesterase inhibition in rats (Cabello et al. 2001). Additionally, there is a 55\%-72\% increase in the odds of Attention-Deficit/Hyperactivity Disorder (ADHD) being diagnosed in children that have ten-fold higher malathion exposure levels (Bouchard et al. 2010). Higher concentrations have also been found to have cytotoxic and genotoxic effects on human and other mammalian cells (Giri et al. 2002; Moore et al. 2010). These effects suggest that increased levels of environmental malathion concentrations are detrimental from a public health perspective.

Ecologically, non-target effects of malathion also have severe negative impacts. To various fish species, malathion is highly toxic, with $\mathrm{LC}_{50}$ values as low as $0.1 \mu \mathrm{g} / \mathrm{L}$ for brown trout (USGA 2000). Many aquatic invertebrates are also highly susceptible to exposure, with $\mathrm{LC}_{50}$ values below environmentally relevant levels, down to $0.5 \mu \mathrm{g} / \mathrm{L}$ (USEPA 2006). It also has other negative effects at sublethal concentrations to a variety of aquatic species (Key et al. 1998; Beauvais et al. 2000; Nguyen and Janssen 2001). Furthermore, the pesticide has been shown to have negative survival effects at environmentally relevant concentrations on tadpoles and frogs (Fordham et al. 2001), small mammals (Newhart 2006), reptiles (Holem et al. 2008), and non-target beneficial insects (Gary and Mussen 1984). It is therefore important that individual species and entire ecosystems are protected from malathion exposure.

Currently, detection of malathion in biological and environmental samples is mostly achieved using gas chromatography (GC). While this method is sensitive and methods for 
malathion detection have been improved upon, it is also time- and labor-intensive and requires expensive and expertly-used equipment (Zweig and Devine 1969; Fournier et al. 1978; Wilson et al. 2003). Other methods of malathion exposure include monitoring blood cholinesterase activity or excretion of metabolites (Fenske and Leffingwell 1989; Wilson et al. 2003). There have also been antibody assays developed for malathion detection, however these antibodies have not been assayed for their affinity or only assayed for cross-reactivity to a few of malathion's major chemically-related metabolites (Nishi et al. 2003; Brun et al. 2005; Vera-Avila et al. 2005). As the antibodies were not identified for their selectivity, it is likely that they bind to major metabolites. This is similar to an antibody which binds to a whole class of molecules similar to malathion (Yan et al. 2009). Therefore, it is necessary to identify a molecule that has been identified for its high affinity and specificity for malathion and not its metabolites.

Identification of such a molecule can be accomplished using the Selective Evolution of Ligands by EXponential Enrichment (SELEX). Binding molecules identified with this process are termed Molecular Recognition Elements (MREs). This in vitro selection process was originally described by two separate laboratories and MREs are selected from a large library $\left(10^{9}-10^{15}\right)$ of different molecules (Ellington and Szostak 1990; Tuerk and Gold 1990). This library is incubated with the target of interest, and those molecules that do not bind to it are removed. Those that do are amplified and can be subjected to negative selections. In those, molecules that do not to the negative targets are amplified. This iterative process continues with increasingly stringent selection conditions until one or a few highly selective molecules with high affinities are identified. A novel variation of this process developed in our laboratory is termed Decoy-SELEX. Using this method, the library is enriched for the target of interest. However, the focus of the selection is on what the MRE should not bind to utilizing stringent 
negative selections. Therefore, the Decoy-SELEX method is ideal for identifying MREs with both high affinity and selectivity for the target of interest.

In this work, the Decoy-SELEX method was utilized to identify an MRE for the pesticide malathion. The selection scheme was designed to enriched a single-stranded DNA (ssDNA) library for molecules which bind to malathion. Those molecules which bound to major metabolites of malathion were removed. Those metabolites included dimethyl thiophosphate (DMTP), dimethyl dithiophosphate (DMDTP), malathion dicarboxylic acid (MDA), and malaoxon (Buratti et al. 2005). The MRE was also designed to not bind to other pesticides found in similar amounts and environments as malathion including propanil (Köck et al. 2010), 2,4-D acid (Baker et al. 2000), and atrazine (McCarthy and Fuiman 2008). Additionally, bovine serum albumin (BSA) was used as a negative target as a model for large, globular proteins. The selected MRE may be used as a sensor in a rapid, field-deployable detection device (Soh et al. 2009; Yang et al. 2010; Lin et al. 2011).

\section{B.2 Methods}

\section{B.2.1 Decoy-SELEX for Malathion-Specific MREs}

Twelve rounds of the Decoy-SELEX process were performed to obtain a MRE specific for the pesticide malathion (Figure B.1). A ssDNA library with a diversity of $10^{15}$ molecules was enriched for those that bound to malathion and not the negative targets of selection (Figure B.2). The library was designed within our laboratory and designated RMW.N34. The ssDNA library was commercially synthesized and consists of two 23 base constant regions for polymerase chain reaction (PCR) amplification that flank a 34 base random region (Eurofins MWG Operon; Huntsville, AL). 
The malathion derivative malathion monocarboxylic acid (Figure B.2b) was covalently biotinylated using EDC-NHS chemistry. It was reacted with 1-ethyl-3-[3dimethylaminopropyl]carbodiimide (EDC) (ThermoScientific; Rockford, IL) and then incubated with Amine-PEG 2 -Biotin (Pierce; Rockford, IL) according to manufacturer's instructions. The biotinylated derivative was bound to streptavidin-coated magnetic beads (New England Biolabs; Ipswich, MA) and washed to provide the immobilized target (IT).

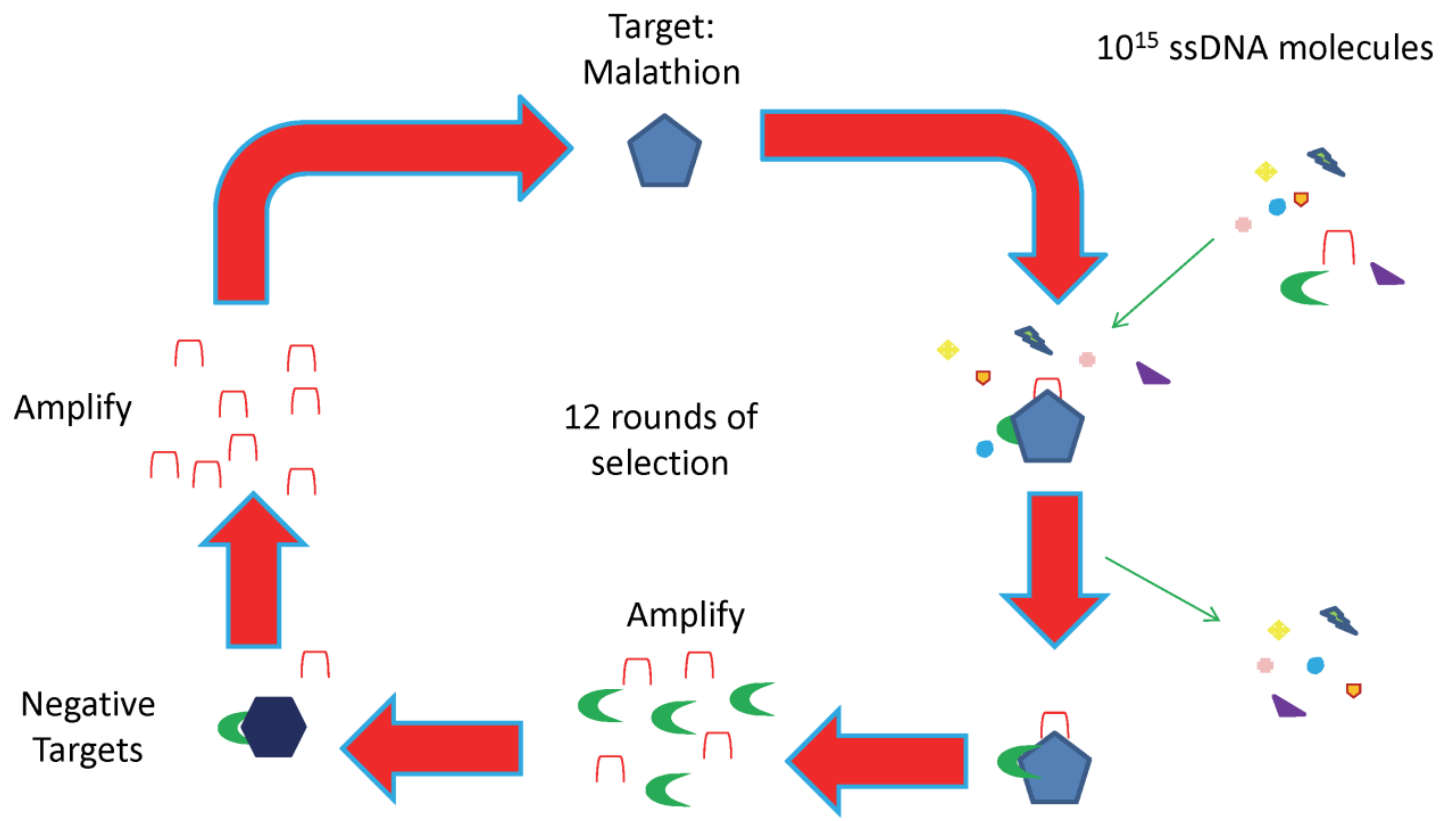

Figure B.1. Decoy-SELEX scheme for selection of a malathion MRE. $10^{15}$ different ssDNA molecules were incubated with malathion. Those that bound were amplified and subjected to closely-related targets. Those do not bind those were amplified and re-subjected to malathion under more stringent conditions. This process continued for 12 rounds.

For Round 1(+) selection, the ssDNA library was incubated with $50 \mu \mathrm{L}$ IT in a total of $500 \mu \mathrm{L}$ selection buffer containing $100 \mathrm{mM}$ sodium chloride, $20 \mathrm{mM}$ Tris- $\mathrm{HCl}$, and $2 \mathrm{mM}$ magnesium chloride (1X selection buffer; SB). The mixture was incubated at room temperature on a rotisserie for 48 hours. The IT with bound ssDNA was removed from the solution by magnetic separation, washed three times with $1 \mathrm{~mL} \mathrm{SB}$, and resuspended in SB. This was used 


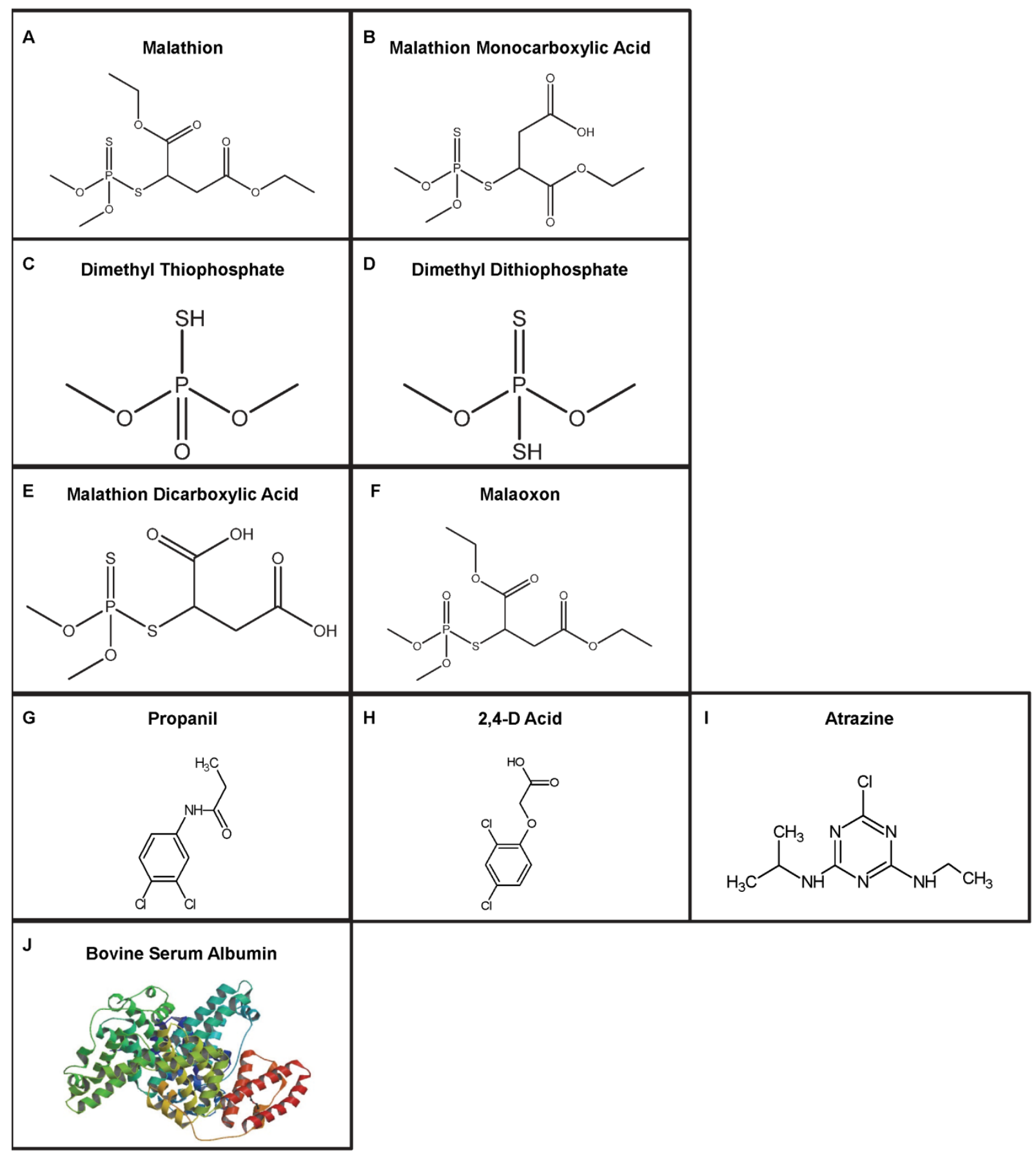

Figure B.2. Chemical structures of molecules used in selection of a malathion-specific MRE. (a) Structure of the target of selection, malathion. (b) Structure of malathion monocarboxylic acid, a malathion derivative used in magnetic bead immobilization. (c) and (d) Structures of dimethyl thiophosphate and dimethyl dithiophosphate used as negative targets in Round 8(-). (e) Chemical structure of malathion dicarboxylic acid, used in Round 9(-) selection. (f) Structure of malaoxon, used in Round 10(-) selection. (g), (h), and (i) Structures of propanil, 2,4-D acid, and atrazine, used successively in Round 7(-) of selection. (j) Ribbon structure of 
bovine serum albumin (BSA) used as a negative target in Round 11(-) of selection (Bujacz 2012).

as a template for PCR amplification of ssDNA bound to the IT. PCR amplification was performed with the following reaction ingredients: enriched ssDNA library, $400 \mathrm{nM}$ forward and biotinylated reverse RMW.N34 primers (Eurofins MWG Operon) (forward: 5'TGTACCGTCTGAGCGATTCGTAC-3', biotinylated reverse: 5’-BiotinGCACTCCTTAACACTGACTGGCT -3'), $250 \mu \mathrm{M}$ deoxynucleotide triphosphates, 1X GoTaq Reaction Buffer (Promega; Madison, WI), 3.5 units Taq, and pure water to $100 \mu \mathrm{L}$. Thermal cycling conditions were: initial denaturation of $95^{\circ} \mathrm{C}$ for 5 minutes; cycling at $95^{\circ} \mathrm{C}$ for 1 minute, $63^{\circ} \mathrm{C}$ for 45 seconds, and $72^{\circ} \mathrm{C}$ for 1 minute; and final extension of $72^{\circ} \mathrm{C}$ for 7 minutes.

Amplification for carrying the library to the next round of selection was performed using either a $4 \mathrm{~mL}$ or a $2 \mathrm{~mL}$ total volume PCR, which respectively preceded positive and negative selection rounds.

The amplified DNA was then purified by agarose gel electrophoresis using the GFX PCR purification kit (GE Healthcare; Piscataway, NJ). Recovered dsDNA was then incubated with streptavidin agarose resin (Pierce) for separation of single strands. The biotinylated reverse primer used in PCR amplification allowed for binding of the reverse strand to the agarose resin. This mixture was placed in a flow-through column and washed with five column volumes of phosphate-buffer saline (PBS). The single forward strand of amplified DNA was eluted from the column using five column volumes of $1 \mathrm{M}$ sodium hydroxide. The elute ssDNA was prepared for ethanol precipitation by adding 0.1 volume of $3 \mathrm{M}$ sodium acetate (pH 5.2), 2.5 volumes of cold $100 \%$ ethanol, and glycogen at $10 \mu \mathrm{g} / \mathrm{mL}$. The solution was frozen and then centrifuged at 13,000xg for 1 hour, the pellet washed with 70\% ethanol, and centrifuged again for 15 minutes. 
The pellet was dried under a vacuum and resuspended in SB. The amount of ssDNA present was verified to be at least $10^{13}$ sequences using a NanoDrop spectrometer (ThermoScientific).

Following Round 2(+) selection, the DNA was prepared for the next round as specified above. However, it was then incubated with immobilization substrate (IS) for Round 2(-) selection for 16 hours in $100 \mu \mathrm{L}$ total volume of SB. To prepare the IS, Amine-PEG 2 -Biotin (Pierce) was incubated with streptavidin-coated magnetic beads (New England Biolabs) and washed to remove unbound biotin reagent. After the incubation period, the IS with bound ssDNA was separated from the solution by magnet. Washes with 25,25 , and $50 \mu \mathrm{L}$ of SB were performed and pooled with the total $100 \mu \mathrm{L}$ of incubation supernatant. This served as a template for PCR amplification, which was performed as previously described. The subtracted library was then prepared for Round 2(+) selection as described above.

Round 2(+) selection proceeded similar to Round 1(+), however the incubation period was 22 hours. Positive selections occurred in this manner, with decreasing incubation period for increased stringency, through Round 6(+). Negative selections similar to Round 1(-) were performed in Round 3 and 5, with longer incubation periods, to remove molecules binding to anything in the incubation that was not the malathion metabolite.

Beginning in Round 7(+), a competitive elution was performed in order to obtain a MRE which bound to malathion in solution. For this, the enriched library was incubated with $50 \mu \mathrm{L}$ of IT in a total volume of $500 \mu \mathrm{L}$ SB for 3 hours. The beads were then washed three times with 1 mL SB. However, in this round, $100 \mu \mathrm{L}$ of $10 \mathrm{mM}$ malathion in SB was then added to the IT and incubated for 5 minutes. The IT was then removed from the solution by magnet, and the supernatant served as the template for PCR amplification. The enriched and amplified library was then prepared for Round 7(-) selection as noted above. This method of selection by 
competitive elution was performed through Round $12(+)$ with decreasing incubation times. Concentrations of malathion used for elution were also decreased, until finally in Round $12\left(^{+}\right)$ the library incubation with the IT was immediate, and elution with $100 \mu \mathrm{L}$ of $100 \mathrm{nM}$ malathion was immediate.

In Round 7(-), competitive elutions were performed with other pesticides found in similar environmental locations as malathion. The enriched library was incubated with the IT, the supernatant removed, and the beads washed 3 times. Then, the beads were incubated for 24 hours at room temperature on the rotisserie with $500 \mu \mathrm{L}$ of $1 \mu \mathrm{M}$ propanil in SB. The supernatant was removed and the beads were washed twice with $1 \mathrm{~mL} \mathrm{SB}$. The IT was then incubated with the same volume and concentration of 2,4-D Acid, washed, incubated with atrazine, and washed. The beads were then resuspended in SB and used as a template in PCR amplification. The DNA was then prepared for the next positive round of selection.

After Round 8(+) selection, Round 8(-) selection was done similar to the previous negative round, however dimethyl thiophosphate, then dimethyl dithiophosphate were used as negative targets. Following Round 9(+), Round 9(-) was performed with $500 \mu \mathrm{L}$ of $1 \mathrm{mM}$ malathion dicarboxylic acid for 15 minutes. Round 10(-) selection was performed with $500 \mu \mathrm{L}$ of $1 \mathrm{mM}$ malaoxon with an incubation period of 1 minute. The final negative selection, Round 11(-), was performed in the same manner with bovine serum albumin as the elutant.

\section{B.2.2 Sequencing of the Enriched ssDNA Library}

Following every third round of selection, a random sample of at least 30 ssDNA molecules present in the enriched library was taken. The library was PCR amplified with nonbiotinylated primers as described above and ligated into the pCR2.1 vector (Invitrogen; Carlsbad, CA) according to manufacturer's instructions. This was cloned into competent bacteria, which 
were plated onto selective agar. Colonies with inserts were amplified, and plasmids containing individual library inserts were purified using the AxyPrep Plasmid Prep Kit (Axygen; Union

Table B.1. Decoy-SELEX outline for identification of malathion MRE

\begin{tabular}{|c|c|c|}
\hline Round & Positive Selection & Negative Selection \\
\hline 1 & Immobilized Target (IT) 48 hrs & Immobilization Substrate (IS) 16 hrs \\
\hline 2 & IT 22 hrs & - \\
\hline 3 & IT $16 \mathrm{hrs}$ & IS $24 \mathrm{hrs}$ \\
\hline 4 & IT $10 \mathrm{hrs}$ & - \\
\hline 5 & IT 6.5 hrs & IS $23 \mathrm{hrs}$ \\
\hline 6 & IT 3 hrs & - \\
\hline 7 & $\begin{array}{l}\text { IT } 3 \text { hrs, } 5 \mathrm{~min} \text { Competitive } \\
\text { Elution (CE) } \mathrm{w} / 10 \mathrm{mM} \\
\text { Malathion }(100 \mathrm{uL})\end{array}$ & $\begin{array}{l}\text { IT } 3 \text { hrs, } 24 \text { hr CE w/ } 1 \mu \mathrm{M} \text { Propanil, } \\
\text { 2,4-D Acid, Atrazine in succession } \\
(500 \mathrm{uL})\end{array}$ \\
\hline 8 & $\begin{array}{l}\text { IT } 1 \text { hr, } 5 \text { min CE w/ } 1 \text { mM Mal } \\
(100 \mathrm{uL})\end{array}$ & $\begin{array}{l}\text { IT } 1 \mathrm{hr}, 24 \mathrm{hr} \text { CE w/ } 1 \mu \mathrm{M} \text { dimethyl } \\
\text { thiophosphate, dimethyl } \\
\text { dithiophosphate in succession ( } 500 \mathrm{uL})\end{array}$ \\
\hline 9 & $\begin{array}{l}\text { IT } 15 \text { min, } 5 \text { min CE w/ } 100 \mathrm{uM} \\
\text { Mal (100 uL) }\end{array}$ & $\begin{array}{l}\text { IT } 15 \text { min, } 15 \text { min CE w/ } 1 \mathrm{mM} \\
\text { Malathion Dicarboxylic Acid (500 uL) }\end{array}$ \\
\hline 10 & $\begin{array}{l}\text { IT } 1 \text { min, } 1 \text { min CE w/ } 10 \mathrm{uM} \\
\text { Mal (100 uL) }\end{array}$ & $\begin{array}{l}\text { IT } 1 \text { min, } 1 \text { min CE w/ } 1 \mathrm{mM} \\
\text { Malaoxon (500 uL) }\end{array}$ \\
\hline 11 & $\begin{array}{l}\text { IT } 1 \text { min, immediate CE w/ } 1 \text { uM } \\
\text { Mal (100 uL) }\end{array}$ & $\begin{array}{l}\text { IT } 1 \text { min, } 15 \text { min CE w/ } 1 \text { mM BSA } \\
(500 \mathrm{uL})\end{array}$ \\
\hline 12 & $\begin{array}{l}\text { IT immediate, immediate CE w/ } \\
100 \mathrm{nM} \text { Mal (100 uL) }\end{array}$ & - \\
\hline
\end{tabular}

Immobilization target (IT) is malathion monocarboxylic acid bound to magnetic beads. Immobilization substrate (IS) is streptavidin-coated magnetic beads with biotin reagent. Competitive elution (CE) is removal of bound ssDNA from IT with target or negative target in solution. Times listed are incubation periods in hours (hrs) or minutes (min).

City, CA). Purified plasmids were sent for sequencing (Eurofins MWG Operon) using the M13R primer complimentary to a region upstream of the pCR2.1 vector. At least 30 sequences 
were obtained for each enriched library. These were then analyzed for consensus sequence families to identify structures binding to malathion.

\section{B.2.3 Determination of MRE Binding Affinity}

The Round 12 sequences were analyzed to produce consensus sequence families, and from those three were chosen for further characterization. These were chosen based on their inclusion in consensus families as well as their structure and stability as predicted by the Mfold DNA web server using buffer salt and $25^{\circ} \mathrm{C}$ as binding conditions (SantaLucia 1998; Peyret 2000; Zuker 2003). The chosen sequences were designated R12.20, R12.14, and R12.29. These sequences were subjected to fluorescent saturation binding assays to determine their affinity for malathion.

Concentrations of $0,0.25,0.5,0.75,1,5,10,15,20$, and $25 \mathrm{nM}$ MRE were used in saturation binding studies. In a $200 \mu \mathrm{L}$ total volume of SB, $10 \mathrm{uL}$ of IT was incubated with each concentration for five minutes. Unbound MRE was removed and each incubation was washed five times with $200 \mu \mathrm{L} \mathrm{SB}$, then the immobilized target was resuspended in $200 \mu \mathrm{L}$ SB and heated to $95^{\circ} \mathrm{C}$ for 10 minutes to denature and elute bound MRE. Eluted ssDNA in SB was placed in a 96-well microplate and measured in a Synergy 2 microplate reader equipped with a tungsten halogen lamp with excitation wavelength of $490 \mathrm{~nm}$ and an emission filter at $520 \mathrm{~nm}$ using Gen5 1.06 software (Biotek US; Winooski, VT). All fluorescence readings on the plate were normalized to $100 \mu \mathrm{L}$ of a $1 \mathrm{nM}$ solution in SB of the fluorescent MRE and the no ssDNA incubation. For each concentration set, the same was done with washed streptavidin-coated magnetic beads to ensure binding over background to the beads. Each set of incubations were performed in triplicate. To determine the dissociation constant $\left(\mathrm{K}_{\mathrm{d}}\right)$ of the MRE, data were analyzed with Origin 8 (OriginLab Corporation; Northampton, MA) using nonlinear regression 
analysis and fit with the equation, $\mathrm{Y}=\left(\left(\mathrm{B}_{\max }{ }^{*} \mathrm{X}\right) /\left(\mathrm{K}_{\mathrm{d}}+\mathrm{X}\right)\right)+\mathrm{NS} * \mathrm{X}$, where $\mathrm{B}_{\max }$ is the maximum binding, $\mathrm{K}_{\mathrm{d}}$ is the dissociation constant, and NS is nonspecific binding as previously described (Langan et al. 2012).

To determine binding of the selected MRE, R12.20, to compounds used in the selection, $50 \mathrm{nM}$ fluorescent ssDNA was used. For each elutant used, in a $200 \mu \mathrm{L}$ total volume of SB, 10 $\mu \mathrm{L}$ IT was incubated with R12.20 for five minutes. The magnetic beads were washed five times with $200 \mu \mathrm{L}$ SB. Then, each incubation was resuspended in $200 \mu \mathrm{L}$ of $1 \mu \mathrm{M}$ in SB of the following in SB: malathion, malathion monocarboxylic acid, dimethyl thiophosphate, dimethyl dithiophosphate, malathion dicarboxylic acid, malaoxon, propanil, 2-4, D acid, atrazine, and BSA. Each elutant was incubated with the immobilized target for ten minutes, and the solution was removed from the beads. This solution was placed in a 96-well plate and measured in a fluorescence plate reader. Data were normalized to an internal fluorescent standard of 1 nMMRE in $100 \mu \mathrm{L}$ SB and background fluorescence of each elution buffer. Each set of binding studies were performed in triplicate. Data were averaged and standard deviations calculated. For each elutant, a one-tailed t-test was performed to determine statistical differences in the means.

\section{B.3 Results and Discussion}

\section{B.3.1 Identification of a Malathion-Specific MRE}

In order to identify a MRE with high affinity for malathion that did not bind to other pesticides or closely-related structures, twelve rounds of Decoy-SELEX were completed (Table B.1). The selection ensured that the final MRE would not bind to the immobilization substrate, propanil, 2,4-D acid, atrazine, dimethyl thiophosphate, dimethyl dithiophosphate, malathion 
R12.13 TGTACCGTCTGAGCGATTCGTACTAAAAATAACCGCCGCAGGTATCTCAGAGGCGCGAGCCAGTCAGTGT TAAGGAGTGC

R12.14 TGTACCGTCTGAGCGATTCGTACTTATTCGTCTTTGTCTTGG-CTCAAAGGCGGTCAGAGCCAGTCAGTGTTAAGGAGTGC

R12.25 TGTACCGTCTGAGCGAT TCGTACATCCGGTCAAGGAAAGG-TATCGCGACAGTCAGAGAGCCAGTCAGTGT TAAGGAGTGC

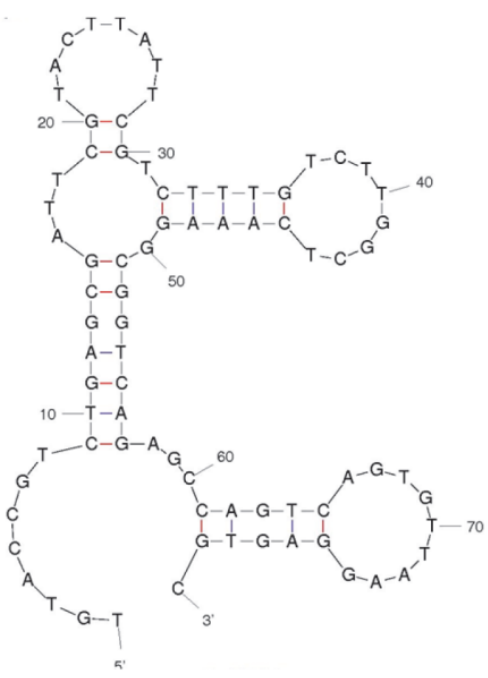

B R12.20 TGTACCGTCTGAGCGATTCGTACTATGGTATCCGAGAGGCCTACGGAATTGTTGTACAGCCAGTCAGTGTTAAGGAGTGC

R12.20 TGTACCGTCT GAGCGATTCGTACTATGGTATCCGAGAGGCCTACGGAATTGTT GTACAGCCAGTCAGTGTTAAGGAGTGC

R12.28 TGTACCGTCTGAGCGATTCGTACCGATTGAATTGGAGAGAGCTACGTCCTGTCTATTAGCCAGTCAGT GTTAAGGAGT GC

R12.46

TGTACCGTCTGAGCGATTCGTACT TAGGGATTTCGCGATCTTGTCCCCTCCAAAGAAGCCAGTCAGT GTTAAGGAGT GC

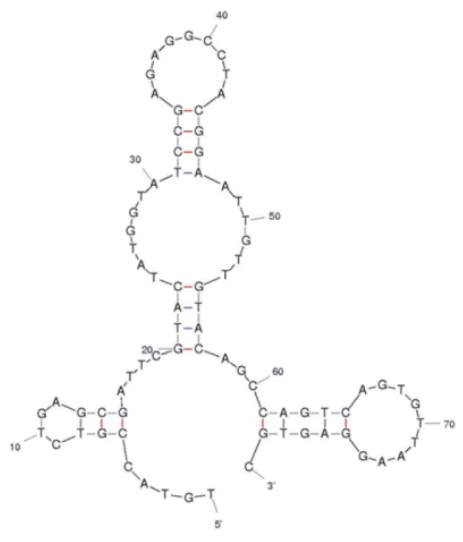

C R12.29 TGTACCGTCTGAGCGATTCGTACT GTGCAGTGGCTCGGACTAGAGTCTCTTCGTCCAGCCAGTCAGTGT TAAGGAGTGC

R12.18 TACCGTCTGAGCGATTCGTACTATATAGCCAGGTAGTATAGTCGGCCTAAAGAGAGCCAGTCAGT GTTAAGGAGTGC

R12.20 TGTACCGTCTGAGCGATTCGTACTATGGTATCCGAGAGGCCTACGGAATTGTTGTACAGCCAGTCAGTGT TAAGGAGTGC

R12.29 TGTACCGTCTGAGCGATTCGTACTGTGCAGTGGCCT-CGGACTAGAGTCTCTTCGTCCAGCCAGTCAGTGTTAAGGAGTGC

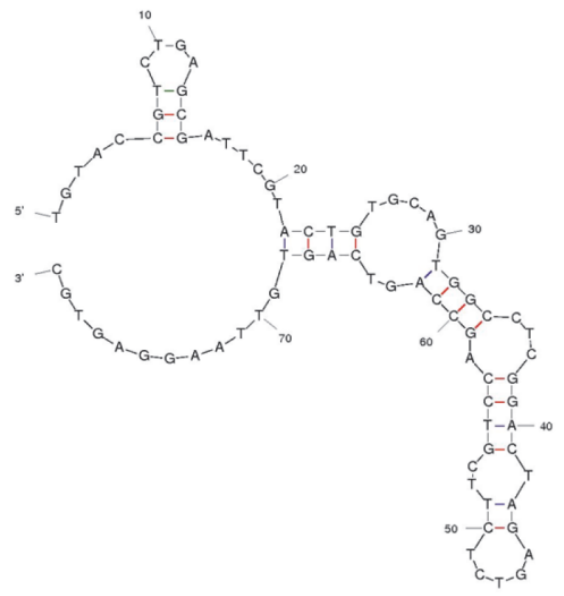


Figure B.3. Sequences of malathion MREs chosen from Round 12 library. For each, the sequence is above an example of a representative consensus sequence family of which the sequence is a member, and the structure as predicted by Mfold is on the bottom (SantaLucia 1998; Peyret 2000; Zuker 2003). Sequence and structure of (a) R12.14, (b) R12.20, and (c) R12.29.

dicarboxylic acid, malaoxon, or bovine serum albumin. Following Rounds 3, 6, 9, and 12, at least 30 random sequences were chosen from the enriched library for consensus sequence analysis. Sequences were analyzed and tracked throughout the in vitro selection. Three sequences were chosen, R12.14, R12.20, and R12.29 based on their presence in consensus families, structure, and stability as predicted by the Mfold DNA web server (Figure B.3). These sequences have a predicted Gibb’s free energy value of $-8.84 \mathrm{kcal} / \mathrm{mol},-5.84 \mathrm{kcal} / \mathrm{mol}$, and -7.89 $\mathrm{kcal} / \mathrm{mol}$, respectively.

\section{B.3.2 Affinity and Specificity of Malathion-Specific MRE}

Fluorescence saturation binding assays identified R12.20 as having the highest affinity and specificity (Figure B.3b). After assaying concentrations in the pM to low nM range, the dissociation constant $\left(\mathrm{K}_{\mathrm{d}}\right)$ of this MRE was determined to be between $1.14 \mathrm{nM}$ and $3.21 \mathrm{nM}$ (Figure B.4). This is in the lower range of previously-selected ssDNA MREs for small-molecule targets (Hamula et al. 2006; Stoltenburg et al. 2007). This high affinity is likely to be a product of the increasingly stringent incubation conditions used in the Decoy-SELEX process. 


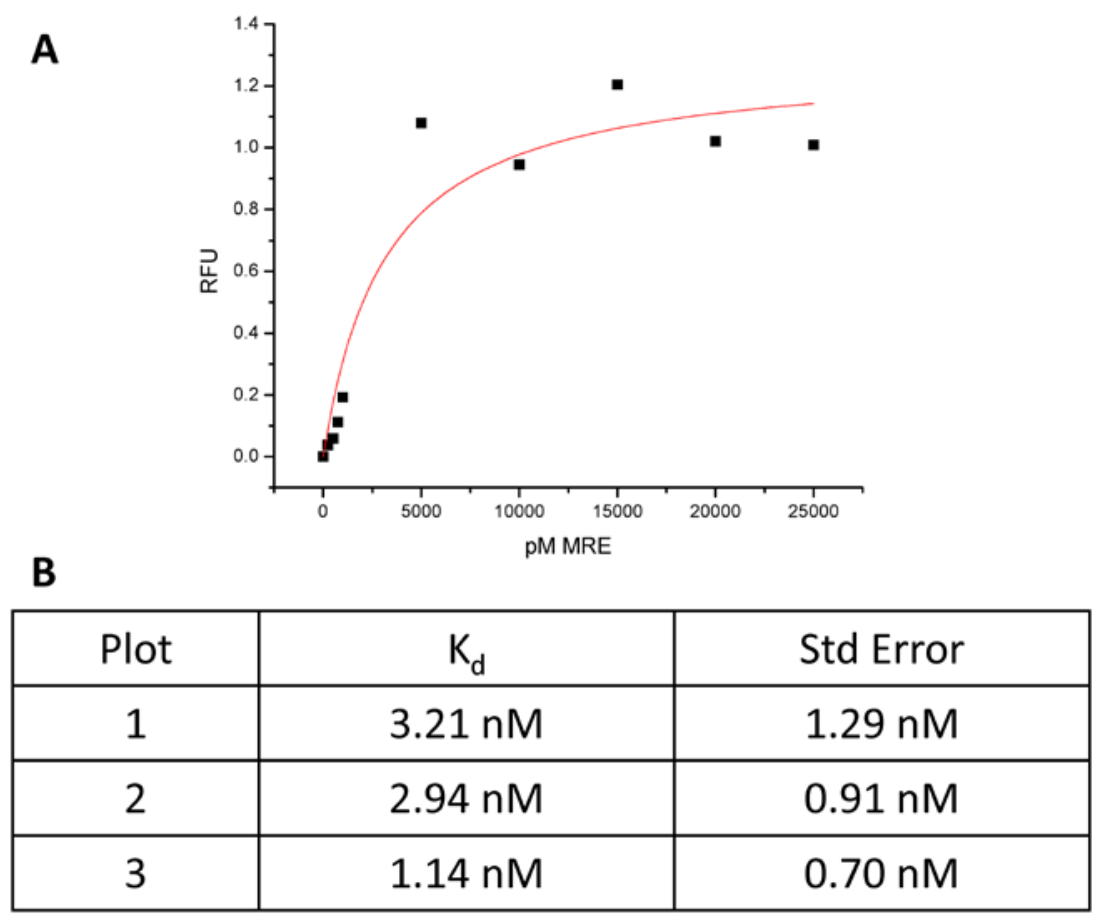

Figure B.4. Fluorescence equilibrium binding assays of R12.20 for malathion. (a) Representative saturation binding curve of R12.23 with nonlinear regression best fit. (b) Equilibrium dissociation constants $\left(\mathrm{K}_{\mathrm{d}}\right)$ with standard error of three R12.23 binding assays.

The cross-reactivity of R12.20 for negative targets used in the selection was also determined. The ssDNA MRE binds to malathion significantly greater than it does to DMTP, DMDTP, malaoxon, propanil, and 2,4-D acid ( $<<0.05$ ) (Fig. 5). The binding of R12.20 to malathion is much higher than it is to atrazine (100\% compared to $49 \%$ ). Large variation, however, render this result statistically insignificant $(\mathrm{p}=0.06)$. Binding to malathion monocarboxylic acid (MMA) is 70\% that of malathion, however there is no difference in these samples $(p=0.06)$. This is expected as MMA was the molecule used to create the immobilized target used in all rounds of selection. Binding of the MRE to malathion dicarboxylic acid (MDA) is $60 \%$ that to malathion, however this is not significantly different $(p=0.11)$. The similarity of MMA and MDA explain the binding of R12.20 to MDA (Fig. 2b and 2e). Binding of the MRE is also as strong to BSA as it is to malathion. This may be due to the large, globular 
nature of the protein and its interaction with ssDNA. This therefore suggests that R12.20 would be most successful as a sensing element in the presence of mostly small molecules. Additionally, even though binding to malathion is approximately two-fold higher than it to DMTP for example, the dissociation constant may be much higher than twice that of R12.20 for malathion. Therefore, incorporation into a sensor may lead to much greater specificity than it appears here.

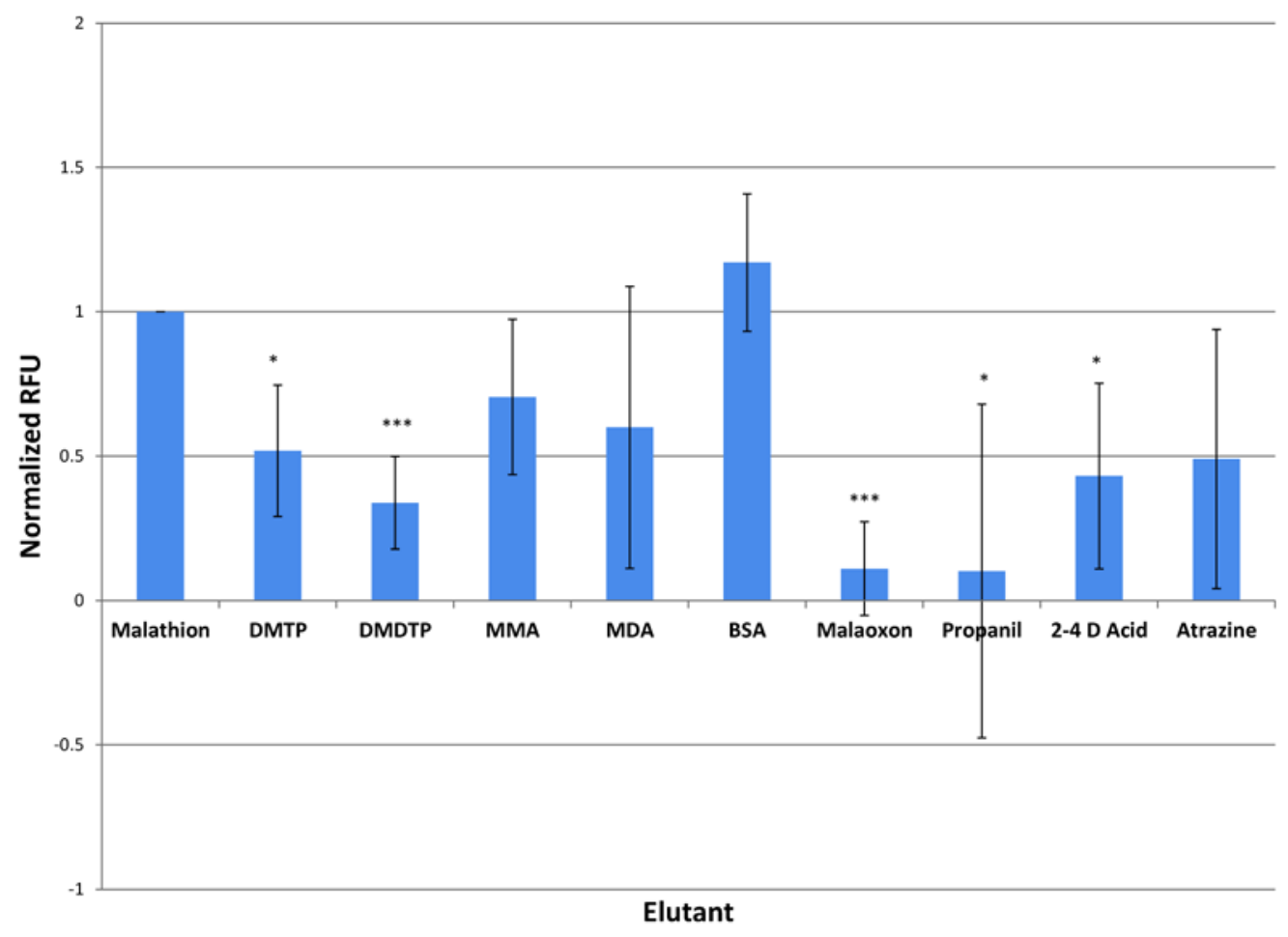

Figure B.5. Cross-binding studies of malathion MRE to other molecules used in the selection. Data were normalized to atrazine binding which was set to $100 \%$, with error bars representing standard deviations. Statistical significance levels of $\mathrm{p}<0.05$ are designated by '*', while levels of $\mathrm{p}<0.01$ are designated by ' $* *$ ', and levels of $\mathrm{p}<0.001$ are designated by ' $* * *$ '. Malathion is the target of the selection and MMA was used to create immobilized target. DMTP, DMDTP, MDA, and malaoxon are molecules similar to malathion used as negative targets. BSA was also used as a negative target as model of a large, globular protein. Propanil, 2,4-D acid, and atrazine are other pesticides which were used as negative targets. MMA = malathion monocarboxylic acid; DMTP = dimethyl thiophosphate; DMDTP = dimethyl dithiophosphate; MDA = malathion dicarboxylic acid; BSA = bovine serum albumin . 
The cross-binding results are particularly interesting as the MRE differentiates malathion from three closely-related structures, including malaoxon, that some antibodies cannot discern between (Yan et al. 2009). It is expected that even though the previously-selected antibodies were not tested for binding affinity or for binding to some of the metabolites tested here, the MRE will have higher affinity and specificity than antibodies previously identified for malathion (Nishi et al. 2003; Brun et al. 2005; Vera-Avila et al. 2005). This is because the in vitro selection process allows the investigator more control over final binding parameters than antibody selection. Furthermore, the Decoy-SELEX process allows the investigator greater control over which closely-related molecules the MRE will bind to.

The selected malathion ssDNA MRE will be useful for environmental sensing. Previously-developed nucleic acid MREs have been used as the sensing element in this capacity (Strehlitz et al. 2012; Yildirim et al. 2012). It is possible that design of the sensor will allow for a rapid, field-usable malathion detection device that will be quicker, cheaper, and easier to operate than current mechanisms. With easier detection of malathion, a greater sampling of contaminated environments will be possible. It is then possible to actively remediate these contaminated environments by biological, chemical, or physical methods (Nair and Pradeep 2007; Xie et al. 2009; Singhal et al. 2012).

\section{B.4 Conclusions}

Decoy-SELEX, a novel variation of in vitro selection, has been utilized to obtain a MRE for the pesticide malathion. Twelve rounds of selection were completed, with consensus sequence families identified and potential MREs obtained. The selected ssDNA MRE, R12.20, has a very high affinity malathion and selectivity compared to various other pesticides and 
metabolites of malathion. This MRE will allow for rapid, cheap, and specific detection of malathion, which will lead to potential remediation of contaminated environments. 


\section{References}

Aalinkeel, R., M. P. N. Nair, et al. (2004). Gene expression of angiogenic factors correlates with metastatic potential of prostate cancer cells. Cancer Res 64(15): 5311-5321.

Abbineni, G., S. Modali, et al. (2010). Evolutionary Selection of New Breast Cancer Cell-Targeting Peptides and Phages with the Cell-Targeting Peptides Fully Displayed on the Major Coat and Their Effects on Actin Dynamics during Cell Internalization. Mol Pharm.

Ackerman, F. (2007). The Economics of Atrazine. Int J Occup Environ Health 13: 441-449.

Adams, G. P., R. Schier, et al. (1998). Increased affinity leads to improved selective tumor delivery of single-chain Fv antibodies. Cancer research 58(3): 485-490.

Ahn, J. Y., M. Jo, et al. (2011). A Sol-Gel-Based Microfluidics System Enhances the Efficiency of RNA Aptamer Selection. Oligonucleotides 21(2): 93-100.

Ajayan, P. M. (1999). Nanotubes from carbon. Chemical Reviews 99(7): 1787-1799.

Ajayan, P. M., J. C. Charlier, et al. (1999). Carbon nanotubes: From macromolecules to nanotechnology. Proc Natl Acad Sci U S A 96(25): 14199-14200.

Albanito, L., R. Lappano, et al. (2008). G-Protein-Coupled Receptor 30 and Estrogen Receptor- $\langle$ Are Involved in the Proliferative Effects Induced by Atrazine in Ovarian Cancer Cells. Environ Health Perspect 116(12): 1648-1655.

Albertorio, F., M. E. Hughes, et al. (2009). Base dependent DNA-carbon nanotube interactions: activation enthalpies and assembly-disassembly control. Nanotechnology 20(39): 395101.

Albertorio, F., M. E. Hughes, et al. (2009). Base dependent DNA-carbon nanotube interactions: activation enthalpies and assembly-disassembly control. Nanotechnology 20(39).

Albertsen, P. C., J. A. Hanley, et al. (2007). 13-year outcomes following treatment for clinically localized prostate cancer in a population based cohort. The Journal of urology 177(3): 932. 
Alpatova, A. L., W. Q. Shan, et al. (2010). Single-walled carbon nanotubes dispersed in aqueous media via non-covalent functionalization: Effect of dispersant on the stability, cytotoxicity, and epigenetic toxicity of nanotube suspensions. Water Research 44(2): 505-520.

Amelinckx, S., X. B. Zhang, et al. (1994). A FORMATION MECHANISM FOR CATALYTICALLY GROWN HELIXSHAPED GRAPHITE NANOTUBES. Science 265(5172): 635-639.

Amistadi, J. K., J. K. Hall, et al. (1997). Comparison of gas chromatography and immunoassay methods for the detection of atrazine in water and soil. J Environ Sci Health B 32(6): 45-60.

Amler, L. C., D. B. Agus, et al. (2000). Dysregulated expression of androgen-responsive and nonresponsive genes in the androgen-independent prostate cancer xenograft model CWR22-R1. Cancer Res 60(21): 6134-6141.

Ando, C., R. Gallavan, et al. (1996). Environmental Monitoring Results of the Mediterranean Fruit Fly Eradication Program, Riverside County 1994. EH95-02.

Ando, Y. and X. L. Zhao (2006). Synthesis of carbon nanotubes by arc-discharge method. New Diamond and Frontier Carbon Technology 16(3): 123-137.

Arnold, M. S., A. A. Green, et al. (2006). Sorting carbon nanotubes by electronic structure using density differentiation. Nat Nanotechnol 1(1): 60-65.

Askonas, B. A., Williams.Ar, et al. (1970). SELECTION OF A SINGLE ANTIBODY-FORMING CELL CLONE AND ITS PROPAGATION IN SYNGENEIC MICE. Proceedings of the National Academy of Sciences of the United States of America 67(3): 1398-\&.

Ator, S. W. (1998). Water Quality in the Potomac River Basin, Maryland, Pennsylvania, Virginia, West Virginia, and the District of Columbia, 1992-96, US Geological Survey.

Ator, S. W. and M. J. Ferrari (1997). Nitrate and selected pesticides in ground water of the Mid-Atlantic Region, US Geological Survey. 
Avouris, P., M. Freitag, et al. (2008). Carbon-nanotube photonics and optoelectronics. Nature Photonics 2(6): 341-350.

Baade, P., M. Coory, et al. (2004). International trends in prostate-cancer mortality: the decrease is continuing and spreading. Cancer causes and control 15(3): 237-241.

Bachilo, S. M., M. S. Strano, et al. (2002). Structure-assigned optical spectra of single-walled carbon nanotubes. Science 298(5602): 2361-2366.

Baddour, C. and C. Briens (2005). Carbon Nanotube Synthesis: A Review. International Journal of Chemical Reactor Engineering 3: R3.

Baker, R. T. K. (1989). CATALYTIC GROWTH OF CARBON FILAMENTS. Carbon 27(3): 315-323.

Baker, S. E., D. B. Barr, et al. (2000). Quantification of selected pesticide metabolites in human urine using isotope dilution high-performance liquid chromatography/tandem mass spectrometry. Journal of Exposure Science and Environmental Epidemiology 10: 789-798.

Bandow, S., S. Asaka, et al. (1998). Effect of the growth temperature on the diameter distribution and chirality of single-wall carbon nanotubes. Physical Review Letters 80(17): 3779-3782.

Barry, M. J. (2009). Screening for prostate cancer-the controversy that refuses to die. New England Journal of Medicine 360(13): 1351-1354.

Baughman, R. H., A. A. Zakhidov, et al. (2002). Carbon nanotubes - the route toward applications. Science 297(5582): 787-792.

Beauvais, S. L., S. B. Jones, et al. (2000). Physiological measures of neurotoxicity of diazinon and malathion to larval rainbow trout (Oncorhynchus mykiss) and their correlation with behavioral measures. Environmental Toxicology and Chemistry 19(7): 1875-1880.

Bello, D., M. Webber, et al. (1997). Androgen responsive adult human prostatic epithelial cell lines immortalized by human papillomavirus 18. Carcinogenesis 18(6): 1215-1223. 
Benedict, C. A., A. J. Mackrell, et al. (1997). Determination of the binding affinity of an anti-CD34 singlechain antibody using a novel, flow cytometry based assay. Journal of immunological methods 201(2): 223-231.

Berens, C., A. Thain, et al. (2001). A tetracycline-binding RNA aptamer. Bioorganic \& Medicinal Chemistry 9(10): 2549-2556.

Berezovski, M. V., M. Lechmann, et al. (2008). Aptamer-facilitated biomarker discovery (AptaBiD). Journal of the American Chemical Society 130(28): 9137-9143.

Berezovski, M. V., M. Lechmann, et al. (2008). Aptamer-facilitated biomarker discovery (AptaBiD). Journal of the American Chemical Society 130(28): 9137-9143.

Bessette, P. H., J. J. Rice, et al. (2004). Rapid isolation of high-affinity protein binding peptides using bacterial display. Protein Eng Des Sel 17(10): 731-739.

Bethune, D. S., C. H. Kiang, et al. (1993). COBALT-CATALYZED GROWTH OF CARBON NANOTUBES WITH SINGLE-ATOMIC-LAYERWALLS. Nature 363(6430): 605-607.

Bhirde, A. A., S. Patel, et al. (2010). Distribution and clearance of PEG-single-walled carbon nanotube cancer drug delivery vehicles in mice. Nanomedicine 5(10): 1535-1546.

Bill-Axelson, A., L. Holmberg, et al. (2008). Radical prostatectomy versus watchful waiting in localized prostate cancer: the Scandinavian prostate cancer group-4 randomized trial. Journal of the National Cancer Institute 100(16): 1144-1154.

Bill-Axelson, A., L. Holmberg, et al. (2011). Radical prostatectomy versus watchful waiting in early prostate cancer. New England Journal of Medicine 364(18): 1708-1717.

Blank, M., T. Weinschenk, et al. (2001). Systematic evolution of a DNA aptamer binding to rat brain tumor microvessels. selective targeting of endothelial regulatory protein pigpen. J Biol Chem 276(19): 16464-16468. 
Boder, E. T., K. S. Midelfort, et al. (2000). Directed evolution of antibody fragments with monovalent femtomolar antigen-binding affinity. Proc Natl Acad Sci U S A 97(20): 10701-10705.

Boder, E. T., K. S. Midelfort, et al. (2000). Directed evolution of antibody fragments with monovalent femtomolar antigen-binding affinity. Proceedings of the National Academy of Sciences 97(20): 10701-10705.

Boder, E. T. and K. D. Wittrup (1997). Yeast surface display for screening combinatorial polypeptide libraries. Nature biotechnology 15(6): 553-557.

Boder, E. T. and K. D. Wittrup (1997). Yeast surface display for screening combinatorial polypeptide libraries. Nat Biotechnol 15(6): 553-557.

Böldicke, T., M. Tesar, et al. (2001). Anti-VEGFR-2 scFvs for Cell Isolation. Single-Chain Antibodies Recognizing the Human Vascular Endothelial Growth Factor Receptor-2 (VEGFR-2/flk-1) on the Surface of Primary Endothelial Cells and Preselected CD34+ Cells from Cord Blood. Stem Cells 19(1): 24-36.

Bouchard, M. F., D. C. Bellinger, et al. (2010). Attention-deficit/hyperactivity disorder and urinary metabolites of organophosphate pesticides. Pediatrics 125(6): e1270-e1277.

Breaker, R. R. and G. F. Joyce (1994). A DNA enzyme that cleaves RNA. Chem Biol 1(4): 223-229.

Brekke, O. H. and I. Sandlie (2003). Therapeutic antibodies for human diseases at the dawn of the twenty-first century. Nat Rev Drug Discov 2(1): 52-62.

Breuer, O. and U. Sundararaj (2004). Big returns from small fibers: A review of polymer/carbon nanotube composites. Polymer Composites 25(6): 630-645.

Brun, E. M., M. Garcés-García, et al. (2005). Evaluation of a novel malathion immunoassay for groundwater and surface water analysis. Environ Sci Technol 39(8): 2786-2794.

Brzezicki, J. M., M. E. Andersen, et al. (2003). Quantitative identification of atrazine and its chlorinated metabolites in plasma. J Anal Toxicol 27(8): 569-573. 
Bujacz, A. (2012). Structures of bovine, equine and leporine serum albumin. Acta Crystallogr D Biol Crystallogr 68(Pt 10): 1278-1289.

Buratti, F. M., A. D'aniello, et al. (2005). Malathion bioactivation in the human liver: the contribution of different cytochrome p450 isoforms. Drug metabolism and disposition 33(3): 295-302.

Burke, D. H., D. C. Hoffman, et al. (1997). RNA aptamers to the peptidyl transferase inhibitor chloramphenicol. Chem Biol 4(11): 833-843.

Cabello, G., M. Valenzuela, et al. (2001). A rat mammary tumor model induced by the organophosphorous pesticides parathion and malathion, possibly through acetylcholinesterase inhibition. Environmental Health Perspectives 109(5): 471.

Carlsson, S., A. J. Vickers, et al. (2012). Prostate cancer screening: facts, statistics, and interpretation in response to the US Preventive Services Task Force review. Journal of Clinical Oncology 30(21): 2581-2584.

Catalona, W., J. Richie, et al. (1994). Comparison of digital rectal examination and serum prostate specific antigen in the early detection of prostate cancer: results of a multicenter clinical trial of 6,630 men. The Journal of urology 151(5): 1283.

Catalona, W. J., D. S. Smith, et al. (1991). Measurement of prostate-specific antigen in serum as a screening test for prostate cancer. New England Journal of Medicine 324(17): 1156-1161.

Cella, L. N., P. Sanchez, et al. (2010). Nano Aptasensor for Protective Antigen Toxin of Anthrax. Analytical Chemistry 82(5): 2042-2047.

Cerchia, L., C. L. Esposito, et al. (2009). Differential SELEX in Human Glioma Cell Lines. PLoS One 4(11). Cerchia, L., J. Hamm, et al. (2002). Nucleic acid aptamers in cancer medicine. FEBS Lett 528(1-3): 12-16. Chang, D. K., C. T. Lin, et al. (2009). A novel peptide enhances therapeutic efficacy of liposomal anticancer drugs in mice models of human lung cancer. PLoS One 4(1): e4171. 
Chao, G., W. L. Lau, et al. (2006). Isolating and engineering human antibodies using yeast surface display. Nat Protoc 1(2): 755-768.

Chavez, J. L., W. Lyon, et al. (2010). Theophylline detection using an aptamer and DNA-gold nanoparticle conjugates. Biosensors \& Bioelectronics 26(1): 23-28.

Chen, H., C. Yu, et al. (2009). A novel near-infrared protein assay based on the dissolution and aggregation of aptamer-wrapped single-walled carbon nanotubes. Chemical Communications(33): 5006-5008.

Chen, Q., J. T. Watson, et al. (2006). Gene expression in the LNCaP human prostate cancer progression model: progression associated expression in vitro corresponds to expression changes associated with prostate cancer progression in vivo. Cancer letters 244(2): 274-288.

Chien, Y. M., F. Lefevre, et al. (2010). A solution processed top emission OLED with transparent carbon nanotube electrodes. Nanotechnology 21(13).

Cho, E. J., J. W. Lee, et al. (2009). Applications of Aptamers as Sensors. Annual Review of Analytical Chemistry. Palo Alto, Annual Reviews. 2: 241-264.

Cho, H., B. R. Baker, et al. (2008). Aptamer-Based SERRS Sensor for Thrombin Detection. Nano Lett 8(12): 4386-4390.

Chochois, V., L. Constans, et al. (2010). Relationships between PSII-independent hydrogen bioproduction and starch metabolism as evidenced from isolation of starch catabolism in the green alga Chlamydomonas reinhardtii. International Journal of Hydrogen Energy 35(19): 10731-10740.

Chou, R., J. M. Croswell, et al. (2011). Screening for prostate cancer: a review of the evidence for the US Preventive Services Task Force. Annals of Internal Medicine 155(11): 762.

Chou, R., T. Dana, et al. (2011). Treatments for localized prostate cancer: systematic review to update the 2002 US Preventive Services Task Force Recommendation. Evidence Synthesis(91). 
Chung, C. H., J. H. Kim, et al. (2012). Nuclease-resistant DNA aptamer on gold nanoparticles for the simultaneous detection of $\mathrm{Pb}(2+)$ and $\mathrm{Hg}(2+)$ in human serum. Biosens Bioelectron.

Codrea, V., M. Hayner, et al. (2010). In vitro selection of RNA aptamers to a small molecule target. Current protocols in nucleic acid chemistry / edited by Serge L. Beaucage ... [et al.] Chapter 9: Unit 9.5.1-23.

Colcher, D., G. Pavlinkova, et al. (1998). Pharmacokinetics and biodistribution of genetically-engineered antibodies. The quarterly journal of nuclear medicine: official publication of the Italian Association of Nuclear Medicine (AIMN)[and] the International Association of Radiopharmacology (IAR) 42(4): 225.

Coleman, J. N., U. Khan, et al. (2006). Small but strong: A review of the mechanical properties of carbon nanotube-polymer composites. Carbon 44(9): 1624-1652.

Conrad, F., X. Zhu, et al. (2009). Human antibodies targeting cell surface antigens overexpressed by the hormone refractory metastatic prostate cancer cells: ICAM-1 is a tumor antigen that mediates prostate cancer cell invasion. Journal of molecular medicine 87(5): 507-514.

Conrad, F., X. Zhu, et al. (2009). Human antibodies targeting cell surface antigens overexpressed by the hormone refractory metastatic prostate cancer cells: ICAM-1 is a tumor antigen that mediates prostate cancer cell invasion. J Mol Med (Berl) 87(5): 507-514.

Cooper, R. L., S. C. Laws, et al. (2007). Atrazine and reproductive function: mode and mechanism of action studies. Birth Defects Res B Dev Reprod Toxicol 80(2): 98-112.

Coupe, R. H., M. A. Manning, et al. (2000). Occurrence of pesticides in rain and air in urban and agricultural areas of Mississippi, April-September 1995. Science of the Total Environment 248(23): $227-240$.

Cui, D. X., F. R. Tian, et al. (2004). Effects of single-walled carbon nanotubes on the polymerase chain reaction. Nanotechnology 15(1): 154-157. 
Cui, Y., H. Urich, et al. (2004). Selection of 2 '-fluoro-modified RNA aptamers for alleviation of cocaine and MK-801 inhibition of the nicotinic acetylcholine receptor. Journal of Membrane Biology 202(3): 137-149.

Dane, K. Y., L. A. Chan, et al. (2006). Isolation of cell specific peptide ligands using fluorescent bacterial display libraries. J Immunol Methods 309(1-2): 120-129.

Daniels, D. A., H. Chen, et al. (2003). A tenascin-C aptamer identified by tumor cell SELEX: systematic evolution of ligands by exponential enrichment. Proc Natl Acad Sci U S A 100(26): 15416-15421.

Daugherty, P. S., G. Chen, et al. (1998). Antibody affinity maturation using bacterial surface display. Protein Eng 11(9): 825-832.

de-los-Santos-Alvarez, N., M. J. Lobo-Castanon, et al. (2007). Modified-RNA aptamer-based sensor for competitive impedimetric assay of neomycin B. Journal of the American Chemical Society 129(13): 3808-+.

Dieckmann, T., E. Suzuki, et al. (1996). Solution structure of an ATP-binding RNA aptamer reveals a novel fold. Rna-a Publication of the Rna Society 2(7): 628-640.

Domingos, R. F., D. F. Simon, et al. (2011). Bioaccumulation and Effects of CdTe/CdS Quantum Dots on Chlamydomonas reinhardtii - Nanoparticles or the Free Ions? Environ Sci Technol 45(18): 76647669.

Dong, L., K. L. Joseph, et al. (2008). Cytotoxicity of single-walled carbon nanotubes suspended in various surfactants. Nanotechnology 19(25).

Du, C. S. and N. Pan (2006). Supercapacitors using carbon nanotubes films by electrophoretic deposition. Journal of Power Sources 160(2): 1487-1494.

El-Mousawi, M., L. Tchistiakova, et al. (2003). A vascular endothelial growth factor high affinity receptor 1-specific peptide with antiangiogenic activity identified using a phage display peptide library. $J$ Biol Chem 278(47): 46681-46691. 
Ellington, A. D. and J. W. Szostak (1990). In vitro selection of RNA molecules that bind specific ligands. Nature 346(6287): 818-822.

Elsässer-Beile, U., G. Reischl, et al. (2009). PET imaging of prostate cancer xenografts with a highly specific antibody against the prostate-specific membrane antigen. Journal of Nuclear Medicine 50(4): 606-611.

Evans, M. J., S. A. Rollins, et al. (1995). In vitro and in vivo inhibition of complement activity by a singlechain Fv fragment recognizing human C5. Mol Immunol 32(16): 1183-1195.

Extra, J. M., F. Rousseau, et al. (1993). Phase I and pharmacokinetic study of Taxotere (RP 56976; NSC 628503) given as a short intravenous infusion. Cancer Res 53(5): 1037-1042.

Fan, W., T. Yanase, et al. (2007). Atrazine-Induced Aromatase Expression Is SF-1 Dependent: Implications for Endocrine Disruption in Wildlife and Reproductive Cancers in Humans. Environ Health Perspect 115(5): 720-727.

Feldhaus, M. J. and R. W. Siegel (2004). Yeast display of antibody fragments: a discovery and characterization platform. J Immunol Methods 290(1-2): 69-80.

Feldhaus, M. J., R. W. Siegel, et al. (2003). Flow-cytometric isolation of human antibodies from a nonimmune Saccharomyces cerevisiae surface display library. Nat Biotechnol 21(2): 163-170.

Feldman, B. J. and D. Feldman (2001). The development of androgen-independent prostate cancer. Nature Reviews Cancer 1(1): 34-45.

Fenske, R. A. and J. T. Leffingwell (1989). Method for the determination of dialkyl phosphate metabolites in urine for studies of human exposure to malathion. J Agric Food Chem 37(4): 995-998.

Ferrari, F., M. Trevisan, et al. (2003). Predicting and measuring environmental concentration of pesticides in air after soil application. Journal of environmental quality 32(5): 1623-1633.

Ferrari, M. J. and M.-A. I. Assessment (1997). Pesticides in surface water of the Mid-Atlantic Region, US Geological Survey. 
Figini, M., L. Obici, et al. (1998). Panning phage antibody libraries on cells: isolation of human Fab fragments against ovarian carcinoma using guided selection. Cancer Res 58(5): 991-996.

Fitch, J. C., S. Rollins, et al. (1999). Pharmacology and biological efficacy of a recombinant, humanized, single-chain antibody C5 complement inhibitor in patients undergoing coronary artery bypass graft surgery with cardiopulmonary bypass. Circulation 100(25): 2499-2506.

Fordham, C. L., J. D. Tessari, et al. (2001). Effects of malathion on survival, growth, development, and equilibrium posture of bullfrog tadpoles (Rana catesbeiana). Environmental Toxicology and Chemistry 20(1): 179-184.

Fournier, E., M. Sonnier, et al. (1978). Detection and assay of organophosphate pesticides in human blood by gas chromatography. Clinical Toxicology 12(4): 457-462.

Franklin, N. R., Y. M. Li, et al. (2001). Patterned growth of single-walled carbon nanotubes on full 4-inch wafers. Applied Physics Letters 79(27): 4571-4573.

Gao, C., S. Mao, et al. (2003). De novo identification of tumor-specific internalizing human antibodyreceptor pairs by phage-display methods. Journal of immunological methods 274(1): 185-197.

Gary, N. E. and E. C. Mussen (1984). Impact of Mediterranean fruit fly malathion bait spray on honey bees. Environmental entomology 13(3): 711-717.

Giebel, L. B., R. T. Cass, et al. (1995). Screening of cyclic peptide phage libraries identifies ligands that bind streptavidin with high affinities. Biochemistry 34(47): 15430-15435.

Gietz, R. and R. Woods (2002). Transformation of yeast by lithium acetate/single-stranded carrier DNA/polyethylene glycol method. Methods in enzymology 350: 87.

Giri, S., S. Prasad, et al. (2002). Genotoxic effects of malathion: an organophosphorus insecticide, using three mammalian bioassays in vivo. Mutation Research/Genetic Toxicology and Environmental Mutagenesis 514(1): 223-231. 
Goda, T. and Y. Miyahara (2012). A hairpin DNA aptamer coupled with groove binders as a smart switch for a field-effect transistor biosensor. Biosensors \& Bioelectronics 32(1): 244-249.

Goertz, P., J. Colin Cox, et al. (2004). Automated selection of aminoglycoside aptamers. JALA 9(3): 150154.

Goldenberg, D. M., H. Goldenberg, et al. (1990). Clinical studies of cancer radioimmunodetection with carcinoembryonic antigen monoclonal antibody fragments labeled with 123 I or 99mTc. Cancer Res 50(3 Suppl): 909s-921s.

Graymore M, S. F., Allinson G (2001). Impacts of Atrazine on Aquatic Ecosystems. Environment International 26: 483-495.

Gugliotti, L. A., D. L. Feldheim, et al. (2004). RNA-mediated metal-metal bond formation in the synthesis of hexagonal palladium nanoparticles. Science 304(5672): 850-852.

Gunneriusson, E., P. Samuelson, et al. (1996). Surface display of a functional single-chain Fv antibody on staphylococci. J Bacteriol 178(5): 1341-1346.

Guo, K. T., G. Ziemer, et al. (2008). CELL-SELEX: Novel perspectives of aptamer-based therapeutics. Int J Mol Sci 9(4): 668-678.

Guo, T., P. Nikolaev, et al. (1995). CATALYTIC GROWTH OF SINGLE-WALLED NANOTUBES BY LASER VAPORIZATION. Chemical Physics Letters 243(1-2): 49-54.

Haddon, R. C., J. Sippel, et al. (2004). Purification and separation of carbon nanotubes. Mrs Bulletin 29(4): 252-259.

Hall, B., J. M. Micheletti, et al. (2009). Design, synthesis, and amplification of DNA pools for in vitro selection. Current protocols in nucleic acid chemistry / edited by Serge L. Beaucage ... [et al.] Chapter 9: Unit 9.2.

Hamula, C. L. A., J. W. Guthrie, et al. (2006). Selection and analytical applications of aptamers. Trends in Analytical Chemistry 25(7): 681-691. 
Harris, E. H. (2001). Chlamydomonas as a model organism. Annual Review of Plant Physiology and Plant Molecular Biology 52: 363-406.

Hasegawa, H., K. Sode, et al. (2008). Selection of DNA aptamers against VEGF(165) using a protein competitor and the aptamer blotting method. Biotechnology Letters 30(5): 829-834.

Hayes, T., K. Haston, et al. (2002). Feminization of Male Frogs in the Wild. Nature 419(895-895).

Hayward, S., R. Dahiya, et al. (1995). Establishment and characterization of an immortalized but nontransformed human prostate epithelial cell line: BPH-1. In Vitro Cellular \& Developmental Biology-Animal 31(1): 14-24.

He, J., Y. Wang, et al. (2010). Targeting prostate cancer cells in vivo using a rapidly internalizing novel human single-chain antibody fragment. Journal of Nuclear Medicine 51(3): 427-432.

He, J. A., Y. A. Liu, et al. (2011). Isolation and Identification of the DNA Aptamer Target to Acetamiprid. J Agric Food Chem 59(5): 1582-1586.

Heller, D. A., S. Baik, et al. (2005). Single-walled carbon nanotube spectroscopy in live cells: Towards long-term labels and optical sensors. Advanced Materials 17(23): 2793-2799.

Herness, E. A. and R. K. Naz (2001). Prostate-specific genes: present status and future direction. Front. Biosci 6: d1083-d1088.

Herranz, S., J. Ram $\tilde{A}^{3} n-A z c \tilde{A}^{3} n$, et al. (2008). Preparation of antibodies and development of a sensitive immunoassay with fluorescence detection for triazine herbicides. Anal Bioanal Chem 391(5): 1801-1812.

Herzberg, M., C. G. Dosoretz, et al. (2004). Simultaneous removal of atrazine and nitrate using a biological granulated activated carbon (BGAC) reactor. Journal of Chemical Technology and Biotechnology 79(6): 626-631.

Ho, M. and I. Pastan (2009). Display and selection of scFv antibodies on HEK-293T cells. Methods Mol Biol 562: 99-113. 
Holem, R. R., W. A. Hopkins, et al. (2008). Effects of repeated exposure to malathion on growth, food consumption, and locomotor performance of the western fence lizard (Sceloporus occidentalis). Environmental Pollution-Kidlington 152(1): 92-98.

Horoszewicz, J. S., E. Kawinski, et al. (1987). Monoclonal antibodies to a new antigenic marker in epithelial prostatic cells and serum of prostatic cancer patients. Anticancer Res 7(5B): 927.

Horoszewicz, J. S., S. S. Leong, et al. (1983). LNCaP model of human prostatic carcinoma. Cancer research 43(4): 1809-1818.

Huang, H. D., M. J. Zou, et al. (2011). Near-infrared fluorescence spectroscopy of single-walled carbon nanotubes and its applications. Trac-Trends in Analytical Chemistry 30(7): 1109-1119.

Huang, H. P., Y. L. Tan, et al. (2010). DNA aptasensor for the detection of ATP based on quantum dots electrochemiluminescence. Nanoscale 2(4): 606-612.

Huang, X. Y., R. S. McLean, et al. (2005). High-resolution length sorting and purification of DNA-wrapped carbon nanotubes by size-exclusion chromatography. Analytical Chemistry 77(19): 6225-6228.

Huizenga, D. E. and J. W. Szostak (1995). A DNA aptamer that binds adenosine and ATP. Biochemistry 34(2): 656-665.

Huse, W. D., L. Sastry, et al. (1989). Generation of a large combinatorial library of the immunoglobulin repertoire in phage lambda. Science 246(4935): 1275-1281.

lijima, S. (1991). Helical Microtubules of Graphitic Carbon. Nature 354(6348): 56-58.

lijima, S. and T. Ichihashi (1993). SINGLE-SHELL CARBON NANOTUBES OF 1-NM DIAMETER. Nature 363(6430): 603-605.

Ireson, C. R. and L. R. Kelland (2006). Discovery and development of anticancer aptamers. Mol Cancer Ther 5(12): 2957-2962.

Ivanov, V., J. B. Nagy, et al. (1994). THE STUDY OF CARBON NANOTUBULES PRODUCED BY CATALYTIC METHOD. Chemical Physics Letters 223(4): 329-335. 
Jackson, G. W., U. Strych, et al. (2009). Portable FRET Sensing of Proteins, Hormones, and Toxins Using DNA Aptamers and Quantum Dots. Boca Raton, Crc Press-Taylor \& Francis Group.

Jackson, H., L. Bacon, et al. (1998). Antigen specificity and tumour targeting efficiency of a human carcinoembryonic antigen-specific scFv and affinity-matured derivatives. British journal of cancer 78(2): 181.

Jacobsen, R. L., T. M. Tritt, et al. (1995). MECHANICAL-PROPERTIES OF VAPOR-GROWN CARBON-FIBER. Carbon 33(9): 1217-1221.

Jain, R. K., D. G. Duda, et al. (2006). Lessons from phase III clinical trials on anti-VEGF therapy for cancer. Nature Clinical Practice Oncology 3(1): 24-40.

Jakobsen, C. G., N. Rasmussen, et al. (2007). Phage display derived human monoclonal antibodies isolated by binding to the surface of live primary breast cancer cells recognize GRP78. Cancer Res 67(19): 9507-9517.

Jayanna, P. K., D. Bedi, et al. (2010). Landscape phage ligands for PC3 prostate carcinoma cells. Protein Eng Des Sel 23(6): 423-430.

Jeng, E. S., A. E. Moll, et al. (2006). Detection of DNA hybridization using the near-infrared band-gap fluorescence of single-walled carbon nanotubes. Nano Lett 6(3): 371-375.

Jiang H, A. C., Graziano N, Roberson A, McGuire M, Khiari D (2006). Occurrence and Removal of Chloro-sTriazines in Water Treatment Plants. Environ Sci Technol 40(11): 3602-3616.

Jiang, M., D. W. Strand, et al. (2009). Functional remodeling of benign human prostatic tissues in vivo by spontaneously immortalized progenitor and intermediate cells. Stem Cells 28(2): 344-356.

Johnston, W. W., C. A. Szpak, et al. (1986). Use of a monoclonal antibody (B72. 3) as a novel immunohistochemical adjunct for the diagnosis of carcinomas in fine needle aspiration biopsy specimens. Human pathology 17(5): 501-513. 
Joseyacaman, M., M. Mikiyoshida, et al. (1993). CATALYTIC GROWTH OF CARBON MICROTUBULES WITH FULLERENE STRUCTURE. Applied Physics Letters 62(2): 202-204.

Journet, C., W. K. Maser, et al. (1997). Large-scale production of single-walled carbon nanotubes by the electric-arc technique. Nature 388(6644): 756-758.

Ju, S. Y., J. Doll, et al. (2008). Selection of carbon nanotubes with specific chiralities using helical assemblies of flavin mononucleotide. Nat Nanotechnol 3(6): 356-362.

Kaighn, M., K. S. Narayan, et al. (1979). Establishment and characterization of a human prostatic carcinoma cell line (PC-3). Investigative urology 17(1): 16.

Kang, J. W., F. T. Nguyen, et al. (2012). Measuring Uptake Dynamics of Multiple Identifiable Carbon Nanotube Species via High-Speed Confocal Raman Imaging of Live Cells. Nano Lett.

Kang, S., M. S. Mauter, et al. (2009). Microbial Cytotoxicity of Carbon-Based Nanomaterials: Implications for River Water and Wastewater Effluent. Environ Sci Technol 43(7): 2648-2653.

Kanwar, J. R., K. Roy, et al. (2011). Chimeric aptamers in cancer cell-targeted drug delivery. Critical Reviews in Biochemistry and Molecular Biology 46(6): 459-477.

Karan, D., D. L. Kelly, et al. (2002). Expression profile of differentially-regulated genes during progression of androgen-independent growth in human prostate cancer cells. Carcinogenesis 23(6): 967-976.

Kase, D., J. L. Kulp, 3rd, et al. (2004). Affinity selection of peptide phage libraries against single-wall carbon nanohorns identifies a peptide aptamer with conformational variability. Langmuir 20(20): 8939-8941.

Kaur, H. and L.-Y. L. Yung (2012). Probing High Affinity Sequences of DNA Aptamer against VEGF165. PLoS One 7(2): e31196.

Kay, B. K., J. Kasanov, et al. (2001). Screening phage-displayed combinatorial peptide libraries. Methods 24(3): 240-246. 
Kelly, K., H. Alencar, et al. (2004). Detection of invasive colon cancer using a novel, targeted, libraryderived fluorescent peptide. Cancer Res 64(17): 6247-6251.

Kelly, K. A. and D. A. Jones (2003). Isolation of a colon tumor specific binding peptide using phage display selection. Neoplasia 5(5): 437-444.

Key, P. B., M. H. Fulton, et al. (1998). Lethal and sublethal effects of malathion on three life stages of the grass shrimp,Palaemonetes pugio. Aquatic Toxicology 40(4): 311-322.

Khandurina, J., T. E. McKnight, et al. (2000). Integrated system for rapid PCR-based DNA analysis in microfluidic devices. Analytical Chemistry 72(13): 2995-3000.

Kim, M., H. J. Um, et al. (2009). Arsenic Removal from Vietnamese Groundwater Using the ArsenicBinding DNA Aptamer. Environ Sci Technol 43(24): 9335-9340.

Kim, M. J., D. W. Shin, et al. (2009). The production of a flexible electroluminescent device on polyethylene terephthalate films using transparent conducting carbon nanotube electrode. Carbon 47(15): 3461-3465.

Kim, S. N., Z. Kuang, et al. (2008). Enrichment of $(6,5)$ single wall carbon nanotubes using genomic DNA. Nano Lett 8(12): 4415-4420.

Kim, Y., C. Liu, et al. (2009). Aptamers generated by Cell SELEX for biomarker discovery. Biomark Med 3(2): 193-202.

Kim, Y. J., Y. S. Kim, et al. (2010). Electrochemical aptasensor for tetracycline detection. Bioprocess and Biosystems Engineering 33(1): 31-37.

Kim, Y. S., C. J. Hyun, et al. (2010). Isolation and characterization of enantioselective DNA aptamers for ibuprofen. Bioorganic \& Medicinal Chemistry 18(10): 3467-3473.

Kim, Y. S., H. S. Jung, et al. (2007). Electrochemical detection of 17 beta-estradiol using DNA aptamer immobilized gold electrode chip. Biosensors \& Bioelectronics 22(11): 2525-2531. 
Köck, M., M. Farré, et al. (2010). Integrated ecotoxicological and chemical approach for the assessment of pesticide pollution in the Ebro River delta (Spain). Journal of Hydrology 383(1): 73-82.

Köhler, A. R., C. Som, et al. (2008). Studying the potential release of carbon nanotubes throughout the application life cycle. Journal of Cleaner Production 16(8): 927-937.

Komatsu, N. and F. Wang (2010). A Comprehensive Review on Separation Methods and Techniques for Single-Walled Carbon Nanotubes. Materials 3(7): 3818-3844.

Kong, J., H. T. Soh, et al. (1998). Synthesis of individual single-walled carbon nanotubes on patterned silicon wafers. Nature 395(6705): 878-881.

Krauland, E. M., B. R. Peelle, et al. (2007). Peptide tags for enhanced cellular and protein adhesion to single-crystalline sapphire. Biotechnol Bioeng 97(5): 1009-1020.

Kronqvist, N., J. Lofblom, et al. (2008). A novel affinity protein selection system based on staphylococcal cell surface display and flow cytometry. Protein Eng Des Sel 21(4): 247-255.

Kroto, H., J. Heath, et al. (1985). C60: Buckminsterfullerene. Nature 318: 162-163.

Krumpe, L. R., A. J. Atkinson, et al. (2006). T7 lytic phage-displayed peptide libraries exhibit less sequence bias than M13 filamentous phage-displayed peptide libraries. Proteomics 6(15): 42104222.

Kucklick, J. R. and T. F. Bidleman (1994). ORGANIC CONTAMINANTS IN WINYAH BAY, SOUTH-CAROLINA

\section{.1. PESTICIDES AND POLYCYCLIC AROMATIC-HYDROCARBONS IN SUBSURFACE AND MICROLAYER} WATERS. Marine Environmental Research 37(1): 63-78.

Kukovitsky, E. F., S. G. L'Vov, et al. (2002). Correlation between metal catalyst particle size and carbon nanotube growth. Chemical Physics Letters 355(5-6): 497-503.

Kupsch, J. M., N. H. Tidman, et al. (1999). Isolation of human tumor-specific antibodies by selection of an antibody phage library on melanoma cells. Clin Cancer Res 5(4): 925-931. 
Kuriyama, M., M. C. Wang, et al. (1980). Quantitation of prostate-specific antigen in serum by a sensitive enzyme immunoassay. Cancer Res 40(12): 4658-4662.

Ladjevardi, S., G. Sandblom, et al. (2010). Tumour grade, treatment, and relative survival in a populationbased cohort of men with potentially curable prostate cancer. Eur Urol 57(4): 631-640.

Ladner, R. C., A. K. Sato, et al. (2004). Phage display-derived peptides as therapeutic alternatives to antibodies. Drug Discov Today 9(12): 525-529.

Lahiji, R. R., B. D. Dolash, et al. (2007). Oligodeoxyribonucleotide Association with Single-Walled Carbon Nanotubes Studied by SPM. Small 3(11): 1912-1920.

Landi, B. J., M. J. Ganter, et al. (2009). Carbon nanotubes for lithium ion batteries. Energy \& Environmental Science 2(6): 638-654.

Langan, T. J., V. T. Nyakubaya, et al. (2012). Assessment of aptamer-steroid binding using stacking-enhanced capillary electrophoresis. Electrophoresis 33(5): 866-869.

Lee, J. F., G. M. Stovall, et al. (2006). Aptamer therapeutics advance. Curr Opin Chem Biol 10(3): 282-289.

LeNoir, J. S., L. L. McConnell, et al. (1999). Summertime transport of current-use pesticides from California's Central Valley to the Sierra Nevada Mountain Range, USA. Environmental Toxicology and Chemistry 18(12): 2715-2722.

Li, J., S. Tan, et al. (2011). Peptide Aptamers with Biological and Therapeutic Applications. Current Medicinal Chemistry 18(27): 4215-4222.

Li, L.-L., Q. Yin, et al. (2012). Polyvalent mesoporous silica nanoparticle-aptamer bioconjugates target breast cancer cells. Advanced healthcare materials 1(5): 567-572.

Li, M., J. M. Zhang, et al. (2012). Detection of Adenosine Triphosphate with an Aptamer Biosensor Based on Surface-Enhanced Raman Scattering. Analytical Chemistry 84(6): 2837-2842.

Li, W. Z., S. S. Xie, et al. (1996). Large-scale synthesis of aligned carbon nanotubes. Science 274(5293): 1701-1703. 
Lin, C. H. and D. J. Patel (1997). Structural basis of DNA folding and recognition in an AMP-DNA aptamer complex: distinct architectures but common recognition motifs for DNA and RNA aptamers complexed to AMP. Chem Biol 4(11): 817-832.

Lin, G. M. (2011). Pexelizumab, an Anti-C5 Complement Antibody for Primary Coronary Revascularization: A New Insight from Old Versions. Cardiovasc Hematol Disord Drug Targets.

Lin, K., J. M. Croswell, et al. (2011). Prostate-specific antigen-based screening for prostate cancer: an evidence update for the US Preventive Services Task Force. Evidence Synthesis(90).

Lin, Q., T. Nguyen, et al. (2011). Microfluidic aptameric affinity sensing of vasopressin for clinical diagnostic and therapeutic applications. Sensors and Actuators B-Chemical 154(1): 59-66.

Liu, B., F. Conrad, et al. (2004). Mapping tumor epitope space by direct selection of single-chain Fv antibody libraries on prostate cancer cells. Cancer Res 64(2): 704-710.

Liu, J. and Y. Lu (2005). Fast colorimetric sensing of adenosine and cocaine based on a general sensor design involving aptamers and nanoparticles. Angewandte Chemie (International ed. in English) 45(1): 90-94.

Liu, S. B., L. Wei, et al. (2009). Sharper and Faster "Nano Darts" Kill More Bacteria: A Study of Antibacterial Activity of Individually Dispersed Pristine Single-Walled Carbon Nanotube. ACS Nano 3(12): 3891-3902.

Liu, Y., N. Tuleouva, et al. (2010). Aptamer-Based Electrochemical Biosensor for Interferon Gamma Detection. Analytical Chemistry 82(19): 8131-8136.

Liu, Z., M. Marquez, et al. (2009). Comparison of protein expression in two prostate cancer cell-lines, LNCaP and DU145, after treatment with somatostatin. Oncology reports 22(6): 1451.

Lou, J. C., M. J. Jung, et al. (2011). Removal of dissolved organic matter (DOM) from raw water by singlewalled carbon nanotubes (SWCNTs). Journal of Environmental Science and Health, Part A 46(12): $1357-1365$. 
Lou, X. H., J. R. Qian, et al. (2009). Micromagnetic selection of aptamers in microfluidic channels. Proc Natl Acad Sci U S A 106(9): 2989-2994.

Lu-Yao, G. L., P. C. Albertsen, et al. (2008). Survival following primary androgen deprivation therapy among men with localized prostate cancer. JAMA: the journal of the American Medical Association 300(2): 173-181.

Lu, Z., E. R. LaVallie, et al. (2003). Using bio-panning of FLITRX peptide libraries displayed on E. coli cell surface to study protein-protein interactions. Methods Mol Biol 205: 267-280.

Lu, Z., K. S. Murray, et al. (1995). Expression of thioredoxin random peptide libraries on the Escherichia coli cell surface as functional fusions to flagellin: a system designed for exploring protein-protein interactions. Biotechnology (N Y) 13(4): 366-372.

Mallikaratchy, P., R. V. Stahelin, et al. (2006). Selection of DNA ligands for protein kinase C-delta. Chemical Communications(30): 3229-3231.

Mandelbaum, R. T., D. L. Allan, et al. (1995). ISOLATION AND CHARACTERIZATION OF A PSEUDOMONAS SP THAT MINERALIZES THE S-TRIAZINE HERBICIDE ATRAZINE. Appl Environ Microbio/ 61(4): 14511457.

Maraj, B., M. Aldersley, et al. (1998). Prostate-specific membrane antigen expression in the duodenum: implications in coeliac disease and immunotherapy for prostate cancer. The Lancet 351(9115): 1559-1560.

Marquis, R., C. Greco, et al. (2008). Supramolecular discrimination of carbon nanotubes according to their helicity. Nano Lett 8(7): 1830-1835.

Matorin, D. N., A. V. Karateyeva, et al. (2010). Influence of Carbon Nanotubes on Chlorophyll Fluorescence Parameters of Green Algae Chlamydomonas reinhardtii. Nanotechnologies in Russia 5(5-6): 320-327. 
McCarthy, I. D. and L. A. Fuiman (2008). Growth and protein metabolism in red drum (Sciaenops ocellatus) larvae exposed to environmental levels of atrazine and malathion. Aquatic Toxicology 88(4): 220-229.

McGuire, M. J., S. Li, et al. (2009). Biopanning of phage displayed peptide libraries for the isolation of cell-specific ligands. Methods Mol Biol 504: 291-321.

McGuire, M. J., K. N. Samli, et al. (2006). Novel ligands for cancer diagnosis: selection of peptide ligands for identification and isolation of B-cell lymphomas. Exp Hematol 34(4): 443-452.

Mills, P. K. (1998). Correlation Analysis of Pesticide Use Data and Cancer Incidence Rates in California Counties. Archives of Environmental Health 53(6): 410-416.

Miyata, Y., T. Kawai, et al. (2007). Chirality-dependent combustion of single-walled carbon nanotubes. Journal of Physical Chemistry C 111(27): 9671-9677.

Mizota, K. and H. Ueda (2006). Endocrine Disrupting Chemical Atrazine Causes Degranulation through Gq/11 Protein-Coupled Neurosteroid Receptor in Mast Cells Toxicol Sci 90(2): 362-368.

Moore, P. D., C. G. Yedjou, et al. (2010). Malathion-induced oxidative stress, cytotoxicity, and genotoxicity in human liver carcinoma (HepG2) cells. Environ Toxicol 25(3): 221-226.

Moore, P. D., C. G. Yedjou, et al. (2010). Malathion-induced oxidative stress, cytotoxicity, and genotoxicity in human liver carcinoma (HepG2) cells. Environmental toxicology 25(3): 221-226.

Morris, K. N., K. B. Jensen, et al. (1998). High affinity ligands from in vitro selection: complex targets. Proc Natl Acad Sci U S A 95(6): 2902-2907.

Moshfeghi, A. A. and C. A. Puliafito (2005). Pegaptanib sodium for the treatment of neovascular agerelated macular degeneration. Expert Opinion on Investigational Drugs 14(5): 671-682.

Mosing, R. K., S. D. Mendonsa, et al. (2005). Capillary electrophoresis-SELEX selection of aptamers with affinity for HIV-1 reverse transcriptase. Analytical Chemistry 77(19): 6107-6112. 
Moyer, V. A. (2012). Screening for prostate cancer: US Preventive Services Task Force recommendation statement. Annals of Internal Medicine.

Muller, S. R., M. Berg, et al. (1997). Atrazine and its primary metabolites in Swiss lakes: Input characteristics and long-term behavior in the water column. Environ Sci Technol 31(7): 21042113.

Nagrath, S., L. V. Sequist, et al. (2007). Isolation of rare circulating tumour cells in cancer patients by microchip technology. Nature 450(7173): 1235-1239.

Nair, A. S. and T. Pradeep (2007). Extraction of chlorpyrifos and malathion from water by metal nanoparticles. J Nanosci Nanotechnol 7(6): 1871-1877.

Navarro, E., F. Piccapietra, et al. (2008). Toxicity of Silver Nanoparticles to Chlamydomonas reinhardtii. Environ Sci Technol 42(23): 8959-8964.

Nelson, A. L. (2010). Antibody fragments Hope and hype. Mabs 2(1): 77-83.

Nelson, A. L. (2010). Antibody fragments: hope and hype. MAbs, Landes Bioscience.

Newhart, K. (2006). Environmental Fate of Malathion. California Environmental Protection Agency

\section{Environmental Monitoring Branch.}

Ng, S. H., J. Wang, et al. (2005). Single wall carbon nanotube paper as anode for lithium-ion battery. Electrochimica Acta 51(1): 23-28.

Nguyen, L. T. H. and C. R. Janssen (2001). Comparative sensitivity of embryo-larval toxicity assays with African catfish (Clarias gariepinus) and zebra fish (Danio rerio). Environmental toxicology 16(6): 566-571.

$\mathrm{Ni}$, X., Y. Zhang, et al. (2011). Prostate-targeted radiosensitization via aptamer-shRNA chimeras in human tumor xenografts. The Journal of clinical investigation 121(6): 2383.

Nielsen, U. B., D. B. Kirpotin, et al. (2006). A novel assay for monitoring internalization of nanocarrier coupled antibodies. BMC immunology 7(1): 24. 
Nishi, K., Y. Imajuku, et al. (2003). Preparation and characterization of monoclonal and recombinant antibodies specific to the insecticide malathion. Journal of Pesticide Science 28(3): 301-309.

Novak, J. P., E. S. Snow, et al. (2003). Nerve agent detection using networks of single-walled carbon nanotubes. Applied Physics Letters 83(19): 4026-4028.

O'Connell, M. J., S. M. Bachilo, et al. (2002). Band gap fluorescence from individual single-walled carbon nanotubes. Science 297(5581): 593-596.

Ocana, C., M. Pacios, et al. (2012). A Reusable Impedimetric Aptasensor for Detection of Thrombin Employing a Graphite-Epoxy Composite Electrode. Sensors 12(3): 3037-3048.

Okamura, K., H. Koike, et al. (2008). Gene Expression Profiles of Prostate Cancer Cell Lines, LNCaP, PC-3 and DU-145, Assessed by cDNA Microarray. Kitakanto Medical Journal 58(4): 363-369.

Ouyang, M., J. L. Huang, et al. (2002). Fundamental electronic properties and applications of singlewalled carbon nanotubes. Acc Chem Res 35(12): 1018-1025.

Oyston, P. C., M. A. Fox, et al. (2009). Novel peptide therapeutics for treatment of infections. J Med Microbiol 58(Pt 8): 977-987.

Ozalp, V. C. (2011). Acoustic quantification of ATP using a quartz crystal microbalance with dissipation. Analyst 136(23): 5046-5050.

Paborsky, L. R., S. N. McCurdy, et al. (1993). The single-stranded DNA aptamer-binding site of human thrombin. J Biol Chem 268(28): 20808-20811.

Pansri, P., N. Jaruseranee, et al. (2009). A compact phage display human scFv library for selection of antibodies to a wide variety of antigens. BMC Biotechnol 9: 6 .

Pantarotto, D., R. Singh, et al. (2004). Functionalized carbon nanotubes for plasmid DNA gene delivery. Angewandte Chemie-International Edition 43(39): 5242-5246. 
Panuwet, P., J. V. Nguyen, et al. (2008). Quantification of atrazine and its metabolites in urine by on-line solid-phase extraction-high-performance liquid chromatography-tandem mass spectrometry. Anal Bioanal Chem 391(5): 1931-1939.

Park, T. J., S. Banerjee, et al. (2006). Purification strategies and purity visualization techniques for singlewalled carbon nanotubes. Journal of Materials Chemistry 16(2): 141-154.

Pasquarell, G. and D. Boyer (1996). Herbicides in karst groundwater in southeast West Virginia. Journal of environmental quality 25(4): 755-765.

Pengfei, Q. F., O. Vermesh, et al. (2003). Toward large arrays of multiplex functionalized carbon nanotube sensors for highly sensitive and selective molecular detection. Nano Lett 3(3): 347351.

Petersen, E. J., L. Zhang, et al. (2011). Potential release pathways, environmental fate, and ecological risks of carbon nanotubes. Environ Sci Technol 45(23): 9837-9856.

Peyret, N. (2000). Prediction of nucleic acid hybridization: parameters and algorithms Detroit. MI Wayne State University PhD Dissertation.

Pienta, K. J. (2001). Preclinical mechanisms of action of docetaxel and docetaxel combinations in prostate cancer. Seminars in oncology, Elsevier.

Pienta, K. J. and D. C. Smith (2005). Advances in Prostate Cancer Chemotherapy: A New Era Begins1. CA: a cancer journal for clinicians 55(5): 300-318.

Pluth, J. M., J. A. Nicklas, et al. (1996). Increased frequency of specific genomic deletions resulting from in vitro malathion exposure. Cancer Res 56(10): 2393-2399.

Pollack, C. E., G. Noronha, et al. (2012). Primary Care Providers' Response to the US Preventive Services Task Force Draft Recommendations on Screening for Prostate Cancer. Archives of internal medicine 172(8): 668 . 
Popov, V. N. (2004). Carbon nanotubes: properties and application. Materials Science \& Engineering RReports 43(3): 61-102.

Popov, V. N., V. E. Van Doren, et al. (2000). Elastic properties of crystals of single-walled carbon nanotubes. Solid State Communications 114(7): 395-399.

Portney, N. G. and M. Ozkan (2006). Nano-oncology: drug delivery, imaging, and sensing. Anal Bioanal Chem 384(3): 620-630.

Potty, A. S. R., K. Kourentzi, et al. (2011). Biophysical characterization of DNA and RNA aptamer interactions with hen egg lysozyme. International Journal of Biological Macromolecules 48(3): 392-397.

Pucca, M. B., T. B. Bertolini, et al. (2011). Therapeutic monoclonal antibodies: scFv patents as a marker of a new class of potential biopharmaceuticals. Brazilian Journal of Pharmaceutical Sciences 47(1): 31-38.

Purcell, M., J. F. Neault, et al. (2001). Interactions of atrazine and 2,4-D with human serum albumin studied by gel and capillary electrophoresis, and FTIR spectroscopy. Biochimica Et Biophysica Acta-Protein Structure and Molecular Enzymology 1548(1): 129-138.

Rasmussen, U. B., V. Schreiber, et al. (2002). Tumor cell-targeting by phage-displayed peptides. Cancer Gene Ther 9(7): 606-612.

Regino, C., K. Wong, et al. (2009). Preclinical evaluation of a monoclonal antibody (3C6) specific for prostate-specific membrane antigen. Current radiopharmaceuticals 2(1): 9.

Renneberg, H., A. Friedetzky, et al. (1999). Prostate specific membrane antigen (PSM) is expressed in various human tissues: implication for the use of PSM reverse transcription polymerase chain reaction to detect hematogenous prostate cancer spread. Urological research 27(1): 23-27.

Rérole, A. L., J. Gobbo, et al. (2011). Peptides and aptamers targeting HSP70: a novel approach for anticancer chemotherapy. Cancer Res 71(2): 484-495. 
Reus, G. Z., S. S. Valvassori, et al. (2008). DNA damage after acute and chronic treatment with malathion in rats. J Agric Food Chem 56(16): 7560-7565.

Rice, J. J., A. Schohn, et al. (2006). Bacterial display using circularly permuted outer membrane protein OmpX yields high affinity peptide ligands. Protein Sci 15(4): 825-836.

Ries, L., C. Kosary, et al., Eds. (1997). SEER Cancer Statistics Review, 1973-1994: Tables and Graphs. NIH Pub 97-2789. Bethesda, MD, National Cancer Institute.

Robert, R., M. J. Jacobin-Valat, et al. (2006). Identification of Human scFvs Targeting Atherosclerotic Lesions. Journal of Biological Chemistry 281(52): 40135-40143.

Robertson, D. H., D. W. Brenner, et al. (1992). ENERGETICS OF NANOSCALE GRAPHITIC TUBULES. Physical Review B 45(21): 12592-12595.

Robertson, M. P., S. M. Knudsen, et al. (2004). In vitro selection of ribozymes dependent on peptides for activity. Rna-a Publication of the Rna Society 10(1): 114-127.

Rodriguez, L. V. and M. K. Terris (1998). Risks and complications of transrectal ultrasound guided prostate needle biopsy: a prospective study and review of the literature. The Journal of urology 160(6): 2115-2120.

Rohr, J. and B. Palmer (2009). Aquatic herbicide exposure increases salamander desiccation risk eight months later in a terrestrial environment. Environ Tox \& Chem 24(5): 1253-1258.

Romanov, V. I., D. B. Durand, et al. (2001). Phage display selection of peptides that affect prostate carcinoma cells attachment and invasion. Prostate 47(4): 239-251.

Sabeti, P. C., P. J. Unrau, et al. (1997). Accessing rare activities from random RNA sequences: The importance of the length of molecules in the starting pool. Chem Bio/ 4(10): 767-774.

Saifuddin, N., A. Z. Raziah, et al. (2013). Carbon Nanotubes: A Review on Structure and Their Interaction with Proteins. Journal of Chemistry. 
Saito, R., M. Fujita, et al. (1992). ELECTRONIC-STRUCTURE OF CHIRAL GRAPHENE TUBULES. Applied Physics Letters 60(18): 2204-2206.

Saltmiras, D. A. and A. T. Lemley (2002). Atrazine degradation by anodic Fenton treatment. Water Research 36(20): 5113-5119.

Salvetat, J. P., G. A. D. Briggs, et al. (1999). Elastic and shear moduli of single-walled carbon nanotube ropes. Physical Review Letters 82(5): 944-947.

SantaLucia, J. (1998). A unified view of polymer, dumbbell, and oligonucleotide DNA nearest-neighbor thermodynamics. Proceedings of the National Academy of Sciences 95(4): 1460-1465.

Sass, J. B. and A. Colangelo (2006). European Union bans atrazine, while the United States negotiates continued use. Int J Occup Environ Health 12(3): 260-267.

Sayer, N., J. Ibrahim, et al. (2002). Structural characterization of a 2'F-RNA aptamer that binds a HIV-1 SU glycoprotein, gp120. Biochem Biophys Res Commun 293(3): 924-931.

Schaffitzel, C., I. Berger, et al. (2001). In vitro generated antibodies specific for telomeric guaninequadruplex DNA react with Stylonychia lemnae macronuclei. Proc Natl Acad Sci U S A 98(15): 8572-8577.

Schier, R., J. Bye, et al. (1996). Isolation of High-affinity Monomeric Human Anti-c-<i> erb $</ i>B-2$ Single chain Fv Using Affinity-driven Selection. Journal of molecular biology 255(1): 28-43.

Schierz, A., A. N. Parks, et al. (2012). Characterization and quantitative analysis of single-walled carbon nanotubes in the aquatic environment using near infrared fluorescence spectroscopy. Environmental Science \& Technology.

Schnorr, J. M. and T. M. Swager (2011). Emerging Applications of Carbon Nanotubes. Chemistry of Materials 23(3): 646-657.

Schrama, D., R. A. Reisfeld, et al. (2006). Antibody targeted drugs as cancer therapeutics. Nature Reviews Drug Discovery 5(2): 147-159. 
Schwab, F., T. D. Bucheli, et al. (2011). Are Carbon Nanotube Effects on Green Algae Caused by Shading and Agglomeration? Environ Sci Technol 45(14): 6136-6144.

Sefah, K., Z. W. Tang, et al. (2009). Molecular recognition of acute myeloid leukemia using aptamers. Leukemia 23(2): 235-244.

Segawa, R., J. Sitts, et al. (1991). Environmental monitoring of malathion aerial applications used to eradicate Mediterranean fruit flies in southern California, 1990. EH91-03.

Senter, P. D. (2009). Potent antibody drug conjugates for cancer therapy. Current opinion in chemical biology 13(3): 235-244.

Shangguan, D., L. Meng, et al. (2008). Identification of liver cancer-specific aptamers using whole live cells. Analytical Chemistry 80(3): 721-728.

Shi, H., Z. W. Tang, et al. (2010). In vivo Fluorescence Imaging of Tumors using Molecular Aptamers Generated by Cell-SELEX. Chemistry-an Asian Journal 5(10): 2209-2213.

Shukla, G. S. and D. N. Krag (2010). Cancer cell-specific internalizing ligands from phage displayed betalactamase-peptide fusion libraries. Protein Eng Des Sel 23(6): 431-440.

Siegel, R., D. Naishadham, et al. (2012). Cancer statistics, 2012. CA: a cancer journal for clinicians.

Sievers, E. L. and M. Linenberger (2001). Mylotarg: antibody-targeted chemotherapy comes of age. Current opinion in oncology 13(6): 522.

Signoretti, S., D. Waltregny, et al. (2000). p63 is a prostate basal cell marker and is required for prostate development. The American journal of pathology 157(6): 1769.

Silver, D. A., I. Pellicer, et al. (1997). Prostate-specific membrane antigen expression in normal and malignant human tissues. Clinical cancer research 3(1): 81-85.

Singhal, R., B. Gangadhar, et al. (2012). Remediation of Malathion Contaminated Soil Using Zero Valent Iron Nano-Particles. American Journal of Analytical Chemistry 3(1): 76-82. 
Smith, P. K., S. K. Shernan, et al. (2011). Effects of C5 complement inhibitor pexelizumab on outcome in high-risk coronary artery bypass grafting: combined results from the PRIMO-CABG I and II trials. J Thorac Cardiovasc Surg 142(1): 89-98.

Snow, E. S., F. K. Perkins, et al. (2005). Chemical detection with a single-walled carbon nanotube capacitor. Science 307(5717): 1942-1945.

So, H. M., D. W. Park, et al. (2008). Detection and titer estimation of Escherichia coli using aptamerfunctionalized single-walled carbon-nanotube field-effect transistors. Small 4(2): 197-201.

So, H. M., K. Won, et al. (2005). Single-walled carbon nanotube biosensors using aptamers as molecular recognition elements. Journal of the American Chemical Society 127(34): 11906-11907.

So, H. M., K. Won, et al. (2005). Single-walled carbon nanotube biosensors using aptamers as molecular recognition elements. Journal of the American Chemical Society 127(34): 11906-11907.

Soh, H. T., J. S. Swensen, et al. (2009). Continuous, Real-Time Monitoring of Cocaine in Undiluted Blood Serum via a Microfluidic, Electrochemical Aptamer-Based Sensor. Journal of the American Chemical Society 131(12): 4262-4266.

Song, S. P., L. H. Wang, et al. (2008). Aptamer-based biosensors. Trac-Trends in Analytical Chemistry 27(2): 108-117.

Sooter, L. and A. Ellington (2004). Automated Selection of Transcription Factor Binding Sites. JALA 9: 277-284.

Stattin, P., E. Holmberg, et al. (2010). Outcomes in localized prostate cancer: National Prostate Cancer Register of Sweden follow-up study. Journal of the National Cancer Institute 102(13): 950-958.

Stebbing, J., E. Copson, et al. (2000). Herceptin (trastuzamab) in advanced breast cancer. Cancer treatment reviews 26(4): 287-290.

Stoger, E., C. Vaquero, et al. (2000). Cereal crops as viable production and storage systems for pharmaceutical scFv antibodies. Plant Mol Biol 42(4): 583-590. 
Stoltenburg, R., C. Reinemann, et al. (2005). FluMag-SELEX as an advantageous method for DNA aptamer selection. Anal Bioanal Chem 383(1): 83-91.

Stoltenburg, R., C. Reinemann, et al. (2007). SELEX--a (r)evolutionary method to generate high-affinity nucleic acid ligands. Biomol Eng 24(4): 381-403.

Stone, K., D. Mickey, et al. (1978). Isolation of a human prostate carcinoma cell line (DU 145). International journal of cancer. Journal international du cancer 21(3): 274.

Strehlitz, B., C. Reinemann, et al. (2012). Aptamers for pharmaceuticals and their application in environmental analytics. Bioanal Rev 4(1): 1-30.

Sueoka, N. (1960). Mitotic Replication of Deoxyribonucleic Acid in Chlamydomonas Reinhardi. Proc Natl Acad Sci U S A 46(1): 83-91.

Sundaram, P., J. Wower, et al. (2012). A nanoscale drug delivery carrier using nucleic acid aptamers for extended release of therapeutic. Nanomedicine-Nanotechnology Biology and Medicine 8(7): 1143-1151.

Suri, C. R., R. Boro, et al. (2009). Immunoanalytical techniques for analyzing pesticides in the environment. Trac-Trends in Analytical Chemistry 28(1): 29-39.

Suzawa, M. and H. Ingraham (2008). The Herbicide Atrazine Activates Endocrine Gene Networks via Non-Steroidal NR5A Nuclear Receptors in Fish and Mammalian Cells. PLoS One 3(5): e2117.

Takahashi, S., H. Mok, et al. (2003). Selection of chronic lymphocytic leukemia binding peptides. Cancer Res 63(17): 5213-5217.

Tans, S. J., A. R. M. Verschueren, et al. (1998). Room-temperature transistor based on a single carbon nanotube. Nature 393(6680): 49-52.

Tavitian, B., F. Duconge, et al. (2009). In Vivo Imaging of Oligonucleotidic Aptamers. Methods in Molecular Biology. G. Mayer, Humana Press Inc, 999 Riverview Dr, Ste 208, Totowa, Nj 075121165 USA. 535: 241-259. 
Tchounwou, T. B., B. A. Wilson, et al. (2001). Atrazine potentiation of arsenic trioxide-induced cytotoxicity and gene expression in human liver carcinoma cells (HepG2). Molecular and Cellular Biochemistry 222: 49-59.

Tewari, A., G. Divine, et al. (2007). Long-term survival in men with high grade prostate cancer: a comparison between conservative treatment, radiation therapy and radical prostatectomy--a propensity scoring approach. Journal of Urology 177(3): 911-915.

Thess, A., R. Lee, et al. (1996). Crystalline ropes of metallic carbon nanotubes. Science 273(5274): 483487.

Thompson, I. M., C. Chi, et al. (2006). Effect of finasteride on the sensitivity of PSA for detecting prostate cancer. J Natl Cancer Inst 98(16): 1128-1133.

Thompson, I. M., C. Chi, et al. (2006). Effect of finasteride on the sensitivity of PSA for detecting prostate cancer. Journal of the National Cancer Institute 98(16): 1128-1133.

Thostenson, E. T., Z. F. Ren, et al. (2001). Advances in the science and technology of carbon nanotubes and their composites: a review. Composites Science and Technology 61(13): 1899-1912.

Tran, C., C. Lin, et al. (2002). Prostate stem cell antigen is a marker of late intermediate prostate epithelial cells. Molecular cancer research: MCR 1(2): 113.

Tu, X. M., S. Manohar, et al. (2009). DNA sequence motifs for structure-specific recognition and separation of carbon nanotubes. Nature 460(7252): 250-253.

Tu, X. M., A. R. H. Walker, et al. (2011). Evolution of DNA Sequences Toward Recognition of Metallic Armchair Carbon Nanotubes. Journal of the American Chemical Society 133(33): 12998-13001.

Tuerk, C. and L. Gold (1990). Systematic Evolution of Ligands by Exponential Enrichment-RNA ligands to Bacteriophage T4 DNA-Polymerase. Science 249(4968): 505-510.

Tuerk, C. and L. Gold (1990). Systematic evolution of ligands by exponential enrichment: RNA ligands to bacteriophage T4 DNA polymerase. Science 249(4968): 505-510. 
Turner, B., J. Waithman, et al. (1991). Environmental monitoring results of the Mexican fruit fly eradication program, San Diego County, spring 1990. California Department of Food and Agriculture Environmental Hazards Assessment Program.

Ulrich, H., J. E. Ippolito, et al. (1998). In vitro selection of RNA molecules that displace cocaine from the membrane-bound nicotinic acetylcholine receptor. Proc Natl Acad Sci U S A 95(24): 1405114056.

Urata, H., K. Nomura, et al. (2007). Fluorescent-labeled single-strand ATP aptamer DNA: Chemo- and enantio-selectivity in sensing adenosine. Biochemical and Biophysical Research Communications 360(2): 459-463.

USDA (2009). Pesticide Data Program, Annual Summary, Calendar Year 2008. U.S. Department of Agriculture.

USEPA (2006). Reregistration Eligibility Decision (RED) for Malathion. U.S. Environmental Protection Agency.

USEPA (2011). Atrazine in Drinking Water. http://water.epa.gov.

USEPA (2012). 2012 Edition of the Drinking Water Standards and Health Advisories. U.S. Environmental Protection Agency EPA 822-S-12-001.

USGA (2000). Organophosphorous pesticide occurrence and distribution in surface and groundwater of the United States, 1992-1997. U.S. Geological Survey, NAWQA Program Report 00-187.

USGS (2002). Pesticide Use Maps. http://water.usgs.gov.

Vaccaro, P., E. Pavoni, et al. (2006). Efficient display of scFv antibodies on bacteriophage lambda. J Immunol Methods 310(1-2): 149-158.

Vally, M., S. Seenu, et al. (2006). Emerging peptide therapeutics for inflammatory diseases. Curr Pharm Biotechnol 7(4): 241-246. 
van den Beucken, T., H. Pieters, et al. (2003). Affinity maturation of Fab antibody fragments by fluorescent-activated cell sorting of yeast-displayed libraries. FEBS Lett 546(2-3): 288-294. Vardharajula, S., S. Z. Ali, et al. (2012). Functionalized carbon nanotubes: biomedical applications. International Journal of Nanomedicine 7: 5361-5374.

Veiseh, O., J. W. Gunn, et al. (2010). Design and fabrication of magnetic nanoparticles for targeted drug delivery and imaging. Advanced drug delivery reviews 62(3): 284-304.

Vera-Avila, L. E., J. C. Vázquez-Lira, et al. (2005). Sol-gel immunosorbents doped with polyclonal antibodies for the selective extraction of malathion and triazines from aqueous samples. Environ Sci Technol 39(14): 5421-5426.

von Minckwitz, G., S. Harder, et al. (2005). Phase I clinical study of the recombinant antibody toxin scFv(FRP5)-ETA specific for the ErbB2/HER2 receptor in patients with advanced solid malignomas. Breast Cancer Res 7(5): R617-626.

Wang, C. L., G. Yang, et al. (2009). In vitro selection of high-affinity DNA aptamers for streptavidin. Acta Biochimica Et Biophysica Sinica 41(4): 335-340.

Wang, J. (2005). Carbon-nanotube based electrochemical biosensors: A review. Electroanalysis 17(1): 714.

Wang, J., Y. Liu, et al. (2011). Selection of phage-displayed peptides on live adherent cells in microfluidic channels. Proc Natl Acad Sci U S A 108(17): 6909-6914.

Wang, J. X., X. Z. Zhang, et al. (2008). Toxicity assessment of manufactured nanomaterials using the unicellular green alga Chlamydomonas reinhardtii. Chemosphere 73(7): 1121-1128.

Wang, L., V. Castranova, et al. (2010). Dispersion of single-walled carbon nanotubes by a natural lung surfactant for pulmonary in vitro and in vivo toxicity studies. Part Fibre Toxicol 7: 31.

Wang, L., X. J. Liu, et al. (2012). Selection of DNA aptamers that bind to four organophosphorus pesticides. Biotechnology Letters 34(5): 869-874. 
Wang, M., A. Liu, et al. (1999). Growth of HPV-18 immortalized human prostatic intraepithelial neoplasia cell lines. Influence of IL-10, follistatin, activin-A, and DHT. Int J Oncol 14(6): 1185-1195.

Wang, M., L. Valenzuela, et al. (1979). Purification of a human prostate specific antigen. Investigative urology 17(2): 159.

Wang, X. X. and E. V. Shusta (2005). The use of scFv-displaying yeast in mammalian cell surface selections. J Immunol Methods 304(1-2): 30-42.

Wang, Y. Y., Y. S. Wang, et al. (2008). Fluorescent detection of ATP based on signaling DNA aptamer attached silica nanoparticles. Nanotechnology 19(41).

Watters, J. M., P. Telleman, et al. (1997). An optimized method for cell-based phage display panning. Immunotechnology 3(1): 21-29.

Weisman, R. B. (2010). Fluorimetric characterization of single-walled carbon nanotubes. Analytical and Bioanalytical Chemistry 396(3): 1015-1023.

Wels, W., I. M. Harwerth, et al. (1992). Selective inhibition of tumor cell growth by a recombinant singlechain antibody-toxin specific for the erbB-2 receptor. Cancer Res 52(22): 6310-6317.

Welsher, K., S. P. Sherlock, et al. (2011). Deep-tissue anatomical imaging of mice using carbon nanotube fluorophores in the second near-infrared window. Proc Natl Acad Sci U S A 108(22): 8943-8948.

Wenseleers, W., I. I. Vlasov, et al. (2004). Efficient isolation and solubilization of pristine single-walled nanotubes in bile salt micelles. Advanced Functional Materials 14(11): 1105-1112.

Willemsen, R., P. Chames, et al. (2008). Selection of human antibody fragments directed against tumor T-cell epitopes for adoptive T-cell therapy. Cytometry A 73(11): 1093-1099.

Williams, K. P., X. H. Liu, et al. (1997). Bioactive and nuclease-resistant L-DNA ligand of vasopressin. Proc Natl Acad Sci U S A 94(21): 11285-11290.

Williams, R. and R. Naz (2010). Novel biomarkers and therapeutic targets for prostate cancer. Frontiers in bioscience (Scholar edition) 2: 677. 
Wilson, D. S. and J. W. Szostak (1999). In vitro selection of functional nucleic acids. Annual Review of Biochemistry 68: 611-647.

Wilson, J., F. Llados, et al. (2003). Toxocological Profile for Malathion. Agency for Toxic Substances and Disease Registry (ATSDR).

Wong, Y. N., N. Mitra, et al. (2006). Survival associated with treatment vs observation of localized prostate cancer in elderly men. JAMA: the journal of the American Medical Association 296(22): 2683-2693.

Woolf, S. H. (1995). Screening for prostate cancer with prostate-specific antigen-an examination of the evidence. New England Journal of Medicine 333(21): 1401-1405.

Wortberg, M., M. H. Goodrow, et al. (1996). Immunoassay for Simazine and Atrazine with Low CrossReactivity for Propazine. J Agric Food Chem 44(8): 2210-2219.

Wright, G. L., C. Haley, et al. (1995). Expression of prostate-specific membrane antigen in normal,

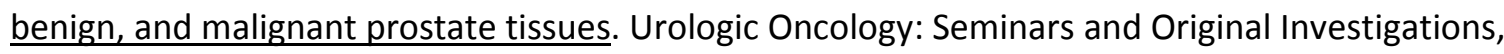
Elsevier.

Wu, Y. R., K. Sefah, et al. (2010). DNA aptamer-micelle as an efficient detection/delivery vehicle toward cancer cells. Proceedings of the National Academy of Sciences of the United States of America 107(1): 5-10.

Xie, S., J. Liu, et al. (2009). Biodegradation of malathion by Acinetobacter johnsonii MA 19 and optimization of cometabolism substrates. Journal of Environmental Sciences(China) 21(1): 76-82.

Yagoda, A. and D. Petrylak (2006). Cytotoxic chemotherapy for advanced hormone-resistant prostate cancer. Cancer 71(S3): 1098-1109.

Yan, L., L. Yang, et al. (2009). Production and characterization of monoclonal antibody for class-specific determination of $\mathrm{O}$, O-dimethyl organophosphorus pesticides and effect of heterologous coating antigens on immunoassay sensitivity. Microchemical Journal 93(1): 36-42. 
Yang, M., M. Loda, et al. (1998). Identification of genes expressed differentially by LNCaP or PC-3 prostate cancer cell lines. Cancer Res 58(16): 3732-3735.

Yang, P. Y., H. X. Wang, et al. (2010). Microfluidic chip-based aptasensor for amplified electrochemical detection of human thrombin. Electrochemistry Communications 12(2): 258-261.

Yao, S. L. and G. Lu-Yao (1999). Population-based study of relationships between hospital volume of prostatectomies, patient outcomes, and length of hospital stay. Journal of the National Cancer Institute 91(22): 1950-1956.

Yi, C. Q., C. C. Fong, et al. (2007). Interactions between carbon nanotubes and DNA polymerase and restriction endonucleases. Nanotechnology 18(2): 025102.

Yildirim, N., F. Long, et al. (2012). Aptamer-based optical biosensor for rapid and sensitive detection of 17beta-estradiol in water samples. Environ Sci Technol 46(6): 3288-3294.

Yoshida, W., E. Mochizuki, et al. (2009). Selection of DNA aptamers against insulin and construction of an aptameric enzyme subunit for insulin sensing. Biosensors \& Bioelectronics 24(5): 1116-1120.

Youn, S., R. Wang, et al. (2011). Mitigation of the impact of single-walled carbon nanotubes on a freshwater green algae: Pseudokirchneriella subcapitata. Nanotoxicology.

Yu, B., M. Ni, et al. (2005). Human scFv antibody fragments specific for hepatocellular carcinoma selected from a phage display library. World J Gastroenterol 11(26): 3985-3989.

Yu, C. C., Y. Hu, et al. (2011). Novel Aptamer-Nanoparticle Bioconjugates Enhances Delivery of Anticancer Drug to MUC1-Positive Cancer Cells In Vitro. PLoS One 6(9).

Yu, M. K., D. Kim, et al. (2011). Image-Guided Prostate Cancer Therapy Using Aptamer-Functionalized Thermally Cross-Linked Superparamagnetic Iron Oxide Nanoparticles. Small 7(15): 2241-2249.

Zhang, G. Y., P. F. Qi, et al. (2006). Selective etching of metallic carbon nanotubes by gas-phase reaction. Science 314(5801): 974-977. 
Zhang, L. B., H. Wei, et al. (2010). A carbon nanotubes based ATP apta-sensing platform and its application in cellular assay. Biosensors \& Bioelectronics 25(8): 1897-1901.

Zhang, X. Q., Q. Guo, et al. (2009). Recent Advances in Nanotechnology Applied to Biosensors. Sensors 9(2): 1033-1053.

Zhang, Y. and L. Zheng (2010). Towards chirality-pure carbon nanotubes. Nanoscale 2(10): 1919-1929.

Zhang, Z. Z., C. C. Shen, et al. (2008). Aqueous suspension of carbon nanotubes enhances the specificity of long PCR. Biotechniques 44(4): 537-544.

Zhang, Z. Z., M. C. Wang, et al. (2007). An aqueous suspension of carbon nanopowder enhances the efficiency of a polymerase chain reaction. Nanotechnology 18(35): 355706.

Zhao, Z., L. Xu, et al. (2009). Recognition of subtype non-small cell lung cancer by DNA aptamers selected from living cells. Analyst 134(9): 1808-1814.

Zheng, M., A. Jagota, et al. (2003). DNA-assisted dispersion and separation of carbon nanotubes. Nature Materials 2(5): 338-342.

Zhou, E. H., R. J. Ellis, et al. (2009). Radiotherapy and survival in prostate cancer patients: a populationbased study. International Journal of Radiation Oncology* Biology* Physics 73(1): 15-23.

Zhou, J. W., A. V. Ellis, et al. (2012). Aptamer sensor for cocaine using minor groove binder based energy transfer. Analytica Chimica Acta 719: 76-81.

Zhu, L., C. Li, et al. (2012). In Vitro Selection of Highly Efficient G-Quadruplex-Based DNAzymes. Analytical Chemistry 84(19): 8383-8390.

Zhu, X. S., L. Zhu, et al. (2009). Acute toxicities of six manufactured nanomaterial suspensions to Daphnia magna. Journal of Nanoparticle Research 11(1): 67-75.

Zitzmann, S., S. Kramer, et al. (2007). Identification and evaluation of a new tumor cell-binding peptide, FROP-1. J Nucl Med 48(6): 965-972. 
Zitzmann, S., W. Mier, et al. (2005). A new prostate carcinoma binding peptide (DUP-1) for tumor imaging and therapy. Clin Cancer Res 11(1): 139-146.

Zuker, M. (2003). Mfold web server for nucleic acid folding and hybridization prediction. Nucleic Acids Res 31(13): 3406-3415.

Zuo, X. L., Y. Xiao, et al. (2009). High Specificity, Electrochemical Sandwich Assays Based on Single Aptamer Sequences and Suitable for the Direct Detection of Small-Molecule Targets in Blood and Other Complex Matrices. Journal of the American Chemical Society 131(20): 6944-+.

Zweig, G. and J. M. Devine (1969). Determination of organophosphorus pesticides in water. Residue reviews 26: 17 . 


\section{Ryan M. Williams}

1 Medical Center Drive

PO Box 9530

Morgantown, WV 26506

\section{Education}

- Graduate

o West Virginia University

August 2008-Present

- Ph.D. Student, Pharmaceutical and Pharmacological Sciences

* August 2010-Present

* Ph.D. Candidate, August 2011

- Ph.D. Student, Department of Biology

* August 2008-July 2010

- Undergraduate

o University of Virginia

August 2004-May 2008

- High School

- B.A., Biology

August 2000-May 2004

o Graham High School, Bluefield, Va.

- Advanced Degree, Summa Cum Laude

\section{Research Experience}

- Graduate Research Assistant

o Dr. Letha Sooter

January 2010-Present

- Investigations in Molecular Recognition Element Selection and SingleWall Carbon Nanotube Properties

o Dr. Rajesh Naz

August 2008-December 2009

- Novel Biomarkers and Immunotherapies for Prostate Cancer

- Undergraduate Research Assistant

o Dr. Edmund Brodie III

September 2006-May 2008

- Evolutionary Genetics of Caribbean Anolis spp.

\section{Fellowships and Assistantships}

- American Foundation for Pharmaceutical Education (AFPE) Pre-Doctoral Fellowship in the Pharmaceutical Sciences

September 1, 2011-August 31, 2013 
- WVNano/NanoSAFE Graduate Fellowship Program (NSF Cooperative Agreement)

August 15, 2011-August 15, 2013

- Cancer Nanotechnology Graduate STEM Fellowship

Selected for period of August-December 2011

- Graduate Research Assistant, West Virginia University

o Dept. of Basic Pharmaceutical Sciences

August 2010-Present

o Dept. of Biology

May 2010-August 2010

o Dept. of Microbiology and Immunology

May 2009-August 2009

- Graduate Teaching Assistant, West Virginia University Dept. of Biology

o Biol 321: The Total Science Experience

Spring 2009, 2010

o Biol 441: Vertebrate Microanatomy

Fall 2009

o Biol 115: Intro Biology Lab

Fall 2008

\section{Publications and Patents}

Williams RM, Hajiran CJ, Nayeem S, and Sooter LJ. Identification of an antibody fragment molecular recognition element specific for prostate cancer cells. In Preparation for Molecular Oncology.

Williams RM, Eamonn Maher, and Sooter LJ. In vitro selection of ssDNA molecular recognition elements for the pesticide malathion. Submitted to Applied Biochemistry and Biotechnology, March 2013.

Williams RM and Sooter LJ. In vitro selection of molecular recognition elements specific for the herbicide atrazine. Submitted to PLoS One, March 2013.

Williams RM, Taylor H, Thomas J, Hines B, and Sooter LJ. The effect of DNA- and sodium cholate-dispersed single wall carbon nanotubes on the green algae Chlamydomonas reinhardtii. Submitted to Environmental Toxicology, March 2013.

Williams RM, Nayeem S, Hines B, and Sooter LJ. The effect of DNA-dispersed single-wall carbon nanotubes on the polymerase chain reaction. Under Review for Nanotechnology. Submitted December 2012. 
Williams RM and Sooter LJ. In vitro selection of cancer cell-specific molecular recognition elements from amino acid libraries. In Preparation for Molecular Cancer Research.

Williams RM and Naz RK. (2010) Novel biomarkers and therapeutic targets for prostate cancer. Frontiers in Bioscience S2:677-684.

Letha J. Sooter and Ryan M. Williams. Single-stranded DNA sequences that bind atrazine and simazine. Disclosure of Intellectual Property, West Virginia University Docket No. 00584. Submitted December 2011.

Letha J. Sooter, Kailey Imlay, and Ryan M. Williams. Single-stranded DNA sequences that bind to Pseudomonas aeruginosa Exotoxin A. Disclosure of Intellectual Property, West Virginia University Docket No. 00585. Submitted December 2011.

\section{Awards and Honors}

- Student Travel Award, American Institute of Chemical Engineers Society for Biological Engineering (AIChE SBE) 2012 International Conference on Bioengineering and Nanotechnology. Berkeley, CA.

June, 2012

- Rho Chi National Pharmacy Honor Society, Alpha Mu Chapter

April, 2012-Present

- George M. Cruise Foundation Scholarship, First Community Bank, Bluefield, WV 2004-2012

- Ethel N. Bowen Foundation Scholarship, First Century Bank, Bluefield, WV 2004-2012

- Cleo Lawson Mitchell Foundation Scholarship, Graham High School, Bluefield, Va. 2004-2008

- Lions Club of Bluefield, Va Scholarship

- Madhu Chopra Memorial Foundation, Bluefield, Va.

- Graham High School Women’s Boosters Foundation Scholarship, Bluefield, Va.

- Peacemaker's Scholarship Foundation, Abingdon, Va.

- Gift of Hope $21^{\text {st }}$ Century Scholars Program, United Methodist Scholarship Fund, Nashville, TN

\section{Laboratory Mentoring Experience}

- 1 High School Student

- 15 Undergraduate Students

- 10 Graduate Rotation Students 
- 4 Graduate Students

- 1 Pharm. D. Student

\section{Oral and Poster Presentations-External}

Williams RM, Hajiran CJ, Nayeem S, and Sooter LJ. In Vitro Selection of Molecular Recognition Elements for Molecular Targeting of Prostate Cancer. EORTC-NCI-AACR Symposium on Molecular Targets and Cancer Therapeutics. Dublin, Ireland. Abstract \# 508. November 2012.

Sooter LJ and Williams RM. Identification and Targeting of Prostate Cancer Cells Via Molecular Recognition Elements. American Institute of Chemical Engineers Society for Biological Engineering (AIChE SBE) 2012 International Conference on Bioengineering and Nanotechnology. Berkeley, CA. June 2012.

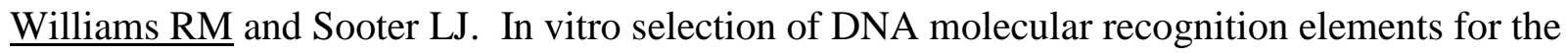
pesticide atrazine. West Virginia Academy of Sciences STaR Symposium, Institute, WV, Oral Presentation. April, 2012.

Williams RM, Nayeem S, and Sooter LJ. In vitro selection of prostate cancer cell-specific molecular recognition elements. American Association of Cancer Researchers (AACR) 2012 Annual Meeting, Chicago, IL. Abstract \# 888. April, 2012.

Williams RM, Nayeem S, and Sooter LJ. Antibody fragments for the targeted treatment and diagnosis of prostate cancer. Appalachian Health Summit, University of Kentucky Center for Clinical and Translational Science. Lexington, KY. March, 2012.

Williams RM, Nayeem S, and Sooter LJ. In Vitro Selection of Prostate Cancer Cell-Specific Molecular Recognition Elements. American Association of Pharmaceutical Scientists (AAPS) Pittsburgh Chapters, Student Research Symposium, Duquesne University. October 2011.

\section{Oral and Poster Presentations-Internal}

Williams RM, Nayeem S, and Sooter LJ. Identification of Antibody Fragments Specific for Prostate Cancer Cells Via SELEX. West Virginia University Linking Innovation, Industry, and Commercialization (LIINC) Biomedical Sciences Graduate Student Event, Invited Oral and Poster Presentation. March 2013. 
Williams RM, Nayeem S, and Sooter LJ In vitro selection of antibody fragments that specifically bind to prostate cancer cells. West Virginia University Health Sciences Center Van Liere Research Day. March 2012.

Williams RM, Nayeem S, and Sooter LJ. Molecular Recognition Elements for Prostate Cancer and Pesticides. West Virginia University Linking Innovation, Industry, and Commercialization (LIINC) Biomedical Sciences Graduate Student Event, Invited Oral and Poster Presentation. February 2012.

Williams RM, Nayeem S, and Sooter LJ. In Vitro Selection of Prostate Cancer Cell and Pesticide Molecular Recognition Elements. West Virginia University School of Pharmacy Research Day. October 2011.

Williams RM and Sooter LJ. Systematic Evolution of Ligands for Prostate Cancer Cells and Atrazine by Exponential Enrichment. West Virginia University Health Sciences Center Van Liere Research Day. March 2011.

Williams RM and Sooter LJ. "Molecular Recognition Elements for Prostate Cancer Cells and Atrazine.” WVNano Brown Bag Lunch Speaker Series Oral Presentation. November 2010.

Williams RM and Sooter LJ. In Vitro Selection of Prostate Cancer Specific and Herbicide Binding Elements. West Virginia University School of Pharmacy Research Day. October 2010.

Williams RM and Sooter LJ. Prostate Cancer Detection by Molecular Recognition Elements. West Virginia Academy of Sciences Symposium. April 2010.

Williams RM and Sooter LJ. Prostate Cancer Detection by Molecular Recognition Elements and Environmental Toxicity of Single-Walled Carbon Nanotubes. West Virginia Nano Symposium. April 2010.

Williams RM and Sooter LJ. Quantum Dot-Conjugated Molecular Recognition Elements in Detecting Prostate Cancer. West Virginia University American Chemical Society Symposium. March 2010.

\section{Other Presentations}

Hajiran CJ, Williams RM, and Sooter LJ. Systematic evolution of ligands for prostate cancer cells and bromacil by exponential enrichment. NanoSAFE SURE Symposium. July 2012. 
Jackson AM, Williams RM, and Sooter LJ. In vitro selection of molecular recognition elements for detection of Exotoxin A and prostate cancer. NanoSAFE REU Symposium. July 2012.

Kulick AR, Williams RM, and Sooter LJ. In Vitro Selection of PETN Molecular Recognition Elements Using Capillary Electrophoresis. West Virginia Academy of Sciences STaR Symposium, Institute, WV. April, 2012.

Imlay K, Williams RM, and Sooter LJ. Isolation of molecular recognition elements against Exotoxin A for application in toxin detection. West Virginia Academy of Sciences STaR Symposium, Institute, WV. April, 2012.

Zimmermann N, Kulick AR, Williams RM, and Sooter LJ. Selections of odor binding Molecular Recognition Elements using CE-SELEX. West Virginia Academy of Sciences STaR Symposium, Institute, WV. April, 2012.

Imlay K, Williams RM, and Sooter LJ. Selection of Molecules that Bind Exotoxin A for Application in Toxin Detection. West Virginia University Health Sciences Center Van Liere Research Day. March 2012.

Kulick AR, Williams RM, and Sooter LJ. In Vitro Selection of PETN Molecular Recognition Elements Using Capillary Electrophoresis. West Virginia University Health Sciences Center Van Liere Research Day. March 2012.

Zimmermann N, Kulick AR, Williams RM, Daly K, and Sooter LJ. In vitro selection of odorspecific aptamers using CE-SELEX. West Virginia University Health Sciences Center Van Liere Research Day. March 2012.

Imlay K, Williams RM, and Sooter LJ. Isolation of Molecules That Bind Exotoxin A for Application in Toxin Detection. Undergraduate Research Day at the Capitol, Charleston, WV. January 2012.

Kulick AR, Williams RM, and Sooter LJ. Identification of Molecules that Bind to the Explosive PETN. Undergraduate Research Day at the Capitol, Charleston, WV. January 2012.

Imlay K, Williams RM, and Sooter LJ. Molecular Recognition Elements against Exotoxin A for Applications in Toxin Detection. West Virginia University School of Pharmacy Research Day. October 2011.

Kulick AR, Williams RM, and Sooter LJ. In Vitro Selection of PETN Molecular Recognition Elements Using Capillary Electrophoresis. West Virginia University School of Pharmacy Research Day. October 2011. 
Sooter LJ, Williams RM, Taylor H, Nayeem S, and Dolash BD. Effects of Single-Walled Carbon Nanotubes on Green Algae and the Polymerase Chain Reaction. West Virginia University School of Pharmacy Research Day. October 2011.

Yedlapalli SL, Williams RM, Holland LM, and Sooter LJ. Two Molecular Recognition Element Selections Against Environmental Pollutants. West Virginia University School of Pharmacy Research Day. October 2011.

Zimmermann N, Kulick AR, Williams RM, Daly K, and Sooter LJ. Identification of OdorantSpecific Aptamers Using CE-SELEX. West Virginia University School of Pharmacy Research Day. October 2011.

Imlay K, Williams RM, and Sooter LJ. In Vitro Selection of Molecular Recognition Elements Against Exotoxin A. WVNano SURE Symposium. July 2011.

DeBerry SM, Williams RM, and Sooter LJ. In Vitro Selection for DNA Aptamers Targeting PETN. West Virginia University Health Sciences Center Van Liere Research Day. March 2011.

Douglas IP, Williams RM, and Sooter LJ. Selecting Molecular Recognition Elements Against HGPIN Cells Using a Yeast Displayed scFv Library. West Virginia University Health Sciences Center Van Liere Research Day. March 2011.

Ghabra O, Williams RM, and Sooter LJ. Forensic Identification Via Hand Bacterial Community Analysis. West Virginia University Health Sciences Center Van Liere Research Day. March 2011.

Imlay K, Williams RM, and Sooter LJ. In Vitro Selection of Molecular Recognition Elements Against Exotoxin A. West Virginia University Health Sciences Center Van Liere Research Day. March 2011

Rhodes GT, Williams RM, and Sooter LJ. A Molecular Recognition Element for the Detection of O,O-Diethyl Dithiophosphate. West Virginia University Health Sciences Center Van Liere Research Day. March 2011.

Taylor H, Williams RM, and Sooter LJ. The Effects of DNA-dispersed and Sodium Cholatedispersed Single-Walled Carbon Nanotubes on Algae Chlamydomonas reinhardtii. West Virginia University Health Sciences Center Van Liere Research Day. March 2011. First place-undergraduate poster competition.

Douglas I, Williams RM, and Sooter LJ.Isolation of Antibody scFv Specific to PIN cells. West Virginia University School of Pharmacy Research Day. October 2010. 
Thomas J, Williams RM, and Sooter LJ.Toxicity of single-walled carbon nanotubes to algae. West Virginia University School of Pharmacy Research Day. October 2010.

\section{Activities}

- Rho Chi National Pharmacy Honor Society, Alpha Mu Chapter

- Member American Association of Cancer Researchers (AACR)

- Member American Association of Pharmaceutical Scientists (AAPS)

o West Virginia University Student Chapter

- Member American Chemical Society (ACS)

- WVU Cell Biology Training Program

- WVNano NanoDays Volunteer Community Outreach

- National Science Foundation Workshop, Science: Becoming the Messenger, West Virginia University, Morgantown, WV

October 11, 2011

- WVU Biology Graduate Student Association Treasurer

- Cavalier Daily Newspaper Associate Sports Editor and Staff Writer

- University of Virginia Children's Hospital Pediatric Unit

o Over 60 volunteer hours

- Energy Express (Americorps) Mentor, Mercer County, WV

o Over 300 volunteer hours per summer ( $>900$ hours total)

Summers 2005, 2006, 2007

- Martha Jefferson Hospital Emergency Department, Charlottesville, Va.

o Over 60 volunteer hours

- University of Virginia Madison House Boosters Program

o Over 30 elementary classroom volunteer hours
2006-2007

2012-Present

2011-Present

2011-Present

2011-2012

2010-2012

2007-2008

2005-2006 
Investigations in Molecular Recognition Element Selection and Single-Wall Carbon Nanotube Properties

$$
\text { Ryan Martin Williams }
$$

A dissertation submitted to the School of Pharmacy at West Virginia University in partial fulfillment of the requirements for the degree of

Doctor of Philosophy in Pharmaceutical and Pharmacological Sciences

Department of Basic Pharmaceutical Sciences

Morgantown, West Virginia

2013

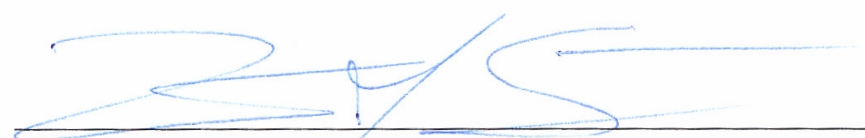

Letha J. Sooter, Ph.D., Chair
Date

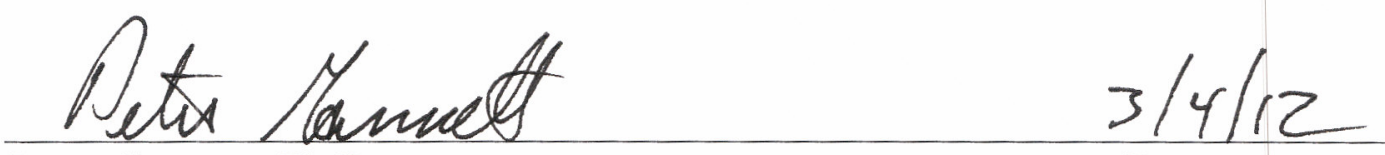

Peter Gannett, Ph.D.

Date

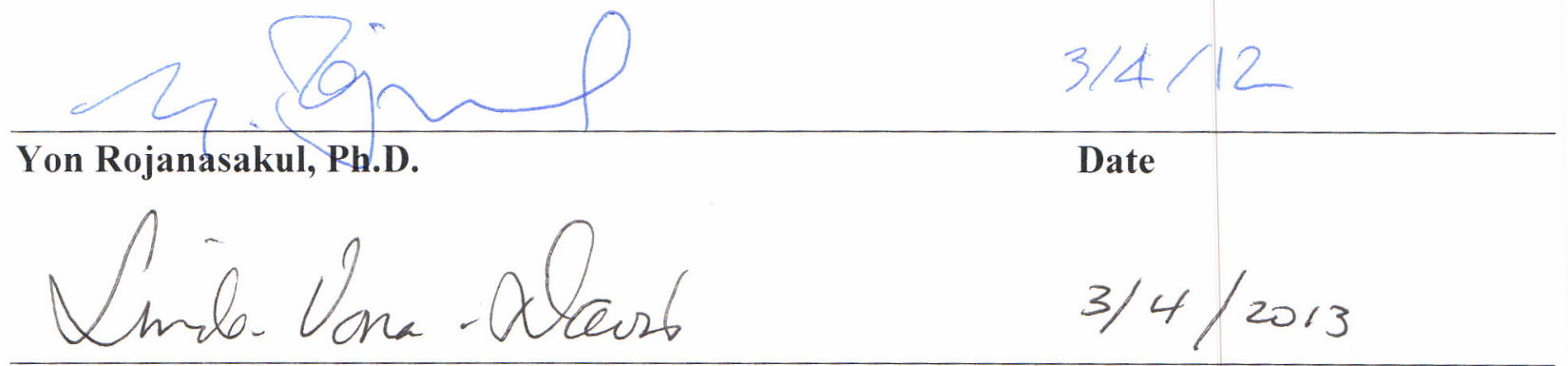

Linda Vona-Davis, Ph.D.

Date

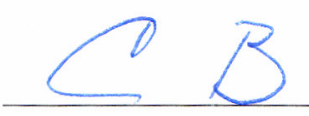

Clifton Bishop, Ph.D.

$3 / 4 / 13$

Date 